\title{
The influence of symbiont diversity on the functional biology of a model sea anemone
}

\author{
Dorota Ewa Starzak
}

A thesis submitted to Victoria University of Wellington in fulfilment of the requirements for the degree of Doctor of Philosophy in Science

Victoria University of Wellington

2012

VICTORIA UNIVERSITY OF WELLINGTON

Te Whare Wānanga o te Ūpoko o te Ika a Māui

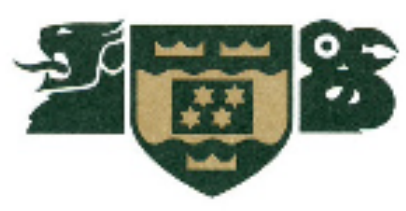



Abstract

\begin{abstract}
Cnidarian-dinoflagellate symbioses, particularly those between anthozoans and dinoflagellates of the genus Symbiodinium (commonly referred to as zooxanthellae) are widespread in the marine environment. They are responsible for the formation of coral reefs and are thus of great ecological importance. In recent years there has been an increase in the frequency and severity of episodes of coral bleaching resulting in degradation and mortality of coral reefs on a global scale. In order to gain a deeper understanding of how corals can adapt to changing environmental conditions, the effect that symbiont type has on the persistence and physiology of an association needs to be ascertained. The aim of this research was to determine how different symbiont types affect the nutritional biology and intracellular physiology of the symbiosis when in association with the sea anemone Aiptasia pulchella. The specific objectives of the study were to; (1) determine whether different symbiont types are equally as adept at supporting the energetic demands of the same host; (2) determine if internal $\mathrm{pH}(\mathrm{pHi})$ is a reflection of symbiont type and whether the optimal $\mathrm{pH}$ for photosynthesis coincides with the host cell $\mathrm{pHi}$; and (3) test the influence of Symbiodinium type on host tissue glycerol and glucose pools.
\end{abstract}

In order to answer these questions, aposymbiotic (i.e. symbiont-free) sea anemones were infected with different Symbiodinium types and the relationship between symbiont type, photosynthetic performance and autotrophic potential was tested. A range of 'normal' and novel cnidarian-dinoflagellate symbioses was also used to measure host cell $\mathrm{pHi}$ and to determine the optimal $\mathrm{pHi}$ of isolated intact symbiosomes (i.e. the vacuoles that house the symbionts), as well as to compare the amounts of free glycerol and glucose (metabolites) present in the host tissues. Different host-symbiont combinations were found to have different photosynthetic and respiratory attributes. Earlier onset of full autotrophy (i.e. when all metabolic carbon demands of the symbiosis were met by photosynthesis) and higher CZAR values (i.e. the contribution of zooxanthellae to animal respiration) were demonstrated by symbioses hosting Symbiodinium B1 both from the original (homologous) and different (heterologous) host. The study showed that 
Abstract

Symbiodinium types differ in their $\mathrm{pH}$ optima and that the optimal $\mathrm{pHi}$ for photosynthesis does not always match the actual measured pHi. Symbiont type was also shown to have an effect on host tissue glycerol and glucose pools, with the associations harbouring the homologous Symbiodinium B1 attaining the highest concentrations of both metabolites.

Findings from this study suggest that corals may be able to maintain an association with a range of Symbiodinium types, and hence potentially switch as a consequence of bleaching. The new symbiont type may not be as nutritionally advantageous as the original type however, which could have implications for the growth and survivorship of the coral, unless it is able to supplement its carbon demands heterotrophically. The rapid proliferation of some of the heterologous Symbiodinium types (e.g. Symbiodinium E2) inside the host indicates that, after bleaching, there is potential for fast symbiont establishment. The reduced carbon contribution of these heterologous symbionts may not be a major concern should the coral be able to reinstate the more nutritionally advantageous symbiont as the dominant type during bleaching recovery. Finally, the rapid proliferation demonstrated by the heterologous Symbiodinium types and the associated metabolic cost to the host, could be an indication of the opportunistic nature of some of these types and may indicate a shift towards parasitism. It is imperative to extend this type of work to corals in the field to determine how these associations behave in nature. Also, in order to get a clearer picture of the diversity in symbiosis physiology, a wider range of Symbiodinium types needs to be investigated. 


\section{Contributions and Publications}

This thesis is written as a series of manuscripts which are to be submitted in the near future. All laboratory work and writing were conducted by the Author.

\section{Chapter 2}

This chapter is formatted as a stand-alone manuscript and is going to be submitted for publication: Starzak, D. E., Quinnell, R. G., Nitschke, M. R., Davy, S. K. (in prep.). The influence of symbiont diversity on the photophysiological performance of novel cnidarian-dinoflagellate symbioses. All laboratory work and analyses were completed by the Author. M. R. Nitschke assisted with data collection for the $A$. pulchella-cultured homologous Symbiodinium association. R. G. Quinnell and S. K. Davy, advised on experimental set-up, analyses and writing.

\section{Chapter 3}

This chapter is formatted as a stand-alone manuscript and is going to be submitted for publication: Starzak, D. E., Gibbin, E., LaFlamme, A. C., Quinnell, R. G., Davy, S. K. (in prep.). The influence of symbiont type on host cell $\mathrm{pH}$ and physiology of a novel cnidarian-algal association. All laboratory work and analyses were completed by the Author. E. Gibbin assisted with the confocal microscopy for the A. pulchellahomologous Symbiodinium association. R. G. Quinnell, A. C. LaFlamme and S. K. Davy, advised on experimental set-up, analyses and writing.

\section{Chapter 4}

This chapter is formatted as a stand-alone manuscript and is going to be submitted for publication: Starzak, D. E., Quinnell, R. G., Davy, S. K. (in prep.). The influence of symbiont type on the glycerol and glucose pools in Aiptasia pulchella. All laboratory work and analyses were completed by the Author. R. G. Quinnell and S. K. Davy, advised on experimental set-up, analyses and writing. 


\section{Acknowledgments}

It would not have been possible to complete this thesis without the help and support of the many kind people who have been here for me during this time, not all of whom I am able to give particular mention to here.

Firstly, I would like to thank the Victoria University of Wellington for awarding me the VUW PhD scholarship and enabling me to come to New Zealand to pursue this $\mathrm{PhD}$ project.

I would like to thank my supervisor, Dr Simon Davy, for his assistance and encouragement throughout the course of my study. His constructive feedback, especially during the writing process, enabled me to put across my findings and ideas with much more insight and clarity. I would also like to thank my second supervisor, Dr Rosanne Quinnel, for all her input; especially the time she spent in the lab with me teaching me some valuable techniques.

Thank you to Dr Anne La Flamme for providing her assistance through the many meetings and emails exchanged during the time Simon was away on sabbatical.

Many thanks go out to the entire Davy lab group. To Dan and Emily for offering your guidance when I first arrived, rather lost and confused. Tom, for your continued willingness to provide experimental advice and assistance (especially confocal related!) and for baby-sitting my anemones so I could go home and visit family. Stefanie, for your HPLC training, your always smiling face and pep talks when things got tough. Emma for our confocal room dance parties (even though you have terrible taste in music). Jen, for being so super organised and allowing me to benefit from it especially when it came to the PCR work. Thomas, for your boundless chemical knowledge and eagerness to help. Paul, for keeping the lab running smoothly and just being entertaining in general. Scott, for being a great lab mate to commiserate with. Finally, a big thanks goes out to Anne - someone I could always rely on not only to help out in the lab (of which there are too many examples to 
mention) but during the day-to-day hurdles too: Thank you for your continued friendship and good heart.

Thank you also to my office mates especially those who have been there for the bulk of my PhD. Rafael and Mark - you both provided some great chats and much needed working breaks.

My parents deserve a huge amount of gratitude. It is through their continued support that I am where I am today. Not only are they always there to call upon in times of financial distress but they can be relied upon for every other eventuality too. My dad is a constant source of knowledge - I have yet to come across a chemical/mathematical/experimental/analytical query for which he does not have an answer. My mom is the most caring person I know - always making sure I have everything I need. Thanks also goes to my grandfather for our Tuesday chats and financial boosts as well as for coming all the way to NZ to visit me. And to my siblings - especially my sister for our interesting “debates".

Thank you to my friends back home for all your love and care. Jen you are the best personal cheer leader I know. Bee - a true friend for life.

A very important and massive thank you goes out to Pierre for being the best partner and best friend. Not only did you make a huge sacrifice to come here with me but you have also had to endure great personal and professional hardships so you could stay by my side. I will always be grateful to you.

Finally thank you to Pilates and the hills and trails of Wellington for keeping me sane. And the post-southerly, blue-sky Wellington day- the perfect reminder that "this too shall pass". 
List of Figures

\section{List of Figures}

Figure 1.1. A schematic diagram representing the continuum that exists between antagonistic and cooperative symbiotic relationships.

Figure 1.2. The symbiosome membrane complex - a transmission electron microscope TEM) and graphic representation.

Figure 1.3. Representation of the daily budget for photosynthetically fixed carbon in a symbiotic cnidarian.

Figure 1.4. Photograph of a partially bleached Acropora palifera colony.

Figure 1.5. Schematic diagram illustrating symbiont (A) "switching" and (B) "shuffling", the two processes that can account for changes in the population of Symbiodinium in a cnidarian host.

Figure 1.6. Photographs of the model anemone, A. pulchella, in the symbiotic (A) and aposymbiotic (B) state.

Figure 2.1. The Mitotic Index (MI) in A. pulchella calculated over a 24 hour period by a range of different cultured heterologous Symbiodinium types

Figure 2.2. Symbiont density and cell-specific gross photosynthesis in $A$. pulchella during infection by a range of different cultured heterologous Symbiodinium types.

Figure 2.3. Gross photosynthesis and total symbiosis respiration in an $A$. pulchella - Symbiodinium symbiosis during infection by a range of different cultured heterologous Symbiodinium types. 
Figure 3.1. A conceptual diagram illustrating the potential relationship between photosynthetic and respiratory activity and internal host $\mathrm{pH}$.

Figure 3.2. Photograph of the step-wise sucrose gradient depicting the $40 \%$ sucrose layer containing the symbiosome enriched fraction and the fluorescence microscope image of the symbiosome enriched fraction showing the symbiosome membrane (golden layer) after staining with FM 1-43.

Figure 3.3. Photograph depicting the experimental $\mathrm{O}_{2}$ flux set-up used to measure the photosynthetic and respiration rates of the isolated symbiosomes at a range of $\mathrm{pH}$ from $\mathrm{pH} 6$ to $\mathrm{ph} 8.5$.

Figure 3.4. Cnidarian endodermal cells isolated from A. pulchella with two intracellular dinoflagellates.

Figure 3.5. Relationship between gross photosynthetic rate $\left(\mathrm{P}_{\text {gross }} ; \mathrm{A}\right)$ and respiration rate $(\mathrm{R} ; \mathrm{B})$ of symbiosomes isolated from $A$. pulchella when in association with three different Symbiodinium types and the $\mathrm{pH}$ of the medium in which they are suspended.

Figure 3.6. Relationship between the ratio of gross photosynthesis ( $\left.\mathrm{P}_{\text {gross }}\right)$ and respiration (R) of symbiosomes isolated from A. pulchella when in association with three different Symbiodinium types and the $\mathrm{pH}$ of the medium in which they are suspended.

Figure 4.1. Concentration of glycerol in tissues of A. pulchella when infected with different Symbiodinium types versus time in the light.

Figure 4.2. Concentration of glucose in tissues of $A$. pulchella when infected with different Symbiodinium types versus time in the light.

102 
List of Figures

Figure 4.3. Concentration of glycerol in A. pulchella tissues when incubated for an hour in the light in the presence of the respiratory inhibitor $\mathrm{NaCN}$.

Figure 4.4. Concentration of glucose in A. pulchella tissues when incubated for an hour in the light in the presence of the respiratory inhibitor $\mathrm{NaCN}$.

Figure 4.5. Concentration of (A) glycerol and (B) glucose in a host tissue homogenate of $A$. pulchella when incubated with different Symbiodinium types in the light.

Figure 5.1. Schematic diagram illustrating the summary of the physiological differences and/or similarities between the $A$. pulchellaSymbiodinium associations investigated in this $\mathrm{PhD}$ study.

Figure A2.1. Calibration curves of pHi of Aiptasia pulchella cells with the SNARF4f signal.

Figure A4.1. Photograph of the step-wise sucrose gradient used to isolate the symbiosome membrane. 


\section{List of Tables}

Table 2.1. Sub-clade, original host species and geographic origin of Symbiodinium cultures.

Table 2.2. The CZAR in A. pulchella 12 weeks after infection with different Symbiodinium types.

Table 2.3. One-way ANOVA results for parameters; Density, MI (Mitotic Index), Volume-specific $\mathrm{P}_{\text {Gross, }}$, Total $\mathrm{P}_{\text {Gross }}, \mathrm{R}_{\mathrm{s}}$ (symbiosis respiration), $\mathrm{R}_{\mathrm{z}}$ (zooxanthellar respiration) and $\mathrm{T}$ (translocation) indicating d.f., $\mathrm{F}$ values and $\mathrm{p}$ values for each Symbiodinium type.

Table 2.4. One-way ANOVA results for parameters; Density, MI (Mitotic Index), Volume-specific $\mathrm{P}_{\text {Gross, }}$, Total $\mathrm{P}_{\text {Gross }}, \mathrm{R}_{\mathrm{s}}$ (symbiosis respiration), $\mathrm{R}_{\mathrm{z}}$ (zooxanthellar respiration) and $\mathrm{T}$ (translocation) indicating d.f., $\mathrm{F}$ values and $p$ values for each time point.

Table A1.1. The CZAR in A. pulchella at Week 1 to Week 12 after infection with different Symbiodinium types.

Table A3.1. NCBI accession numbers of the ITS2 sequences that were used to identify the Symbiodinium types used in this study. 


\section{Abbreviations}

2-D: $\quad$ two dimensional

ANOVA: analysis of variance

APX: $\quad$ ascorbate peroxidase

ATP: adenosine triphosphate

CO2: $\quad$ carbon dioxide

CZAR: fractional contribution of translocated zooxanthellar carbon to the host's daily respiratory carbon requirements

DAPI: 6-diamidino-2-phenylindole dihydrochloride, 2-(4-amidinophenyl)-6indolecarbamidine dihydrochloride

DIC: dissolved inorganic carbon

DMSO: dimethyl sulfoxide

FAAs: $\quad$ free amino acids

FITC: fluorescein isothiocyanate

FSW: $\quad 0.44 \mu \mathrm{m}$ filtered seawater

$\mathrm{HCl}: \quad$ hydrochloric acid

$\mathrm{HCO}_{3}{ }^{-}: \quad$ bicarbonate

HRF: $\quad$ host release factor

ITS: internal transcribed spacer

LSD: least squares difference

MI: $\quad$ mitotic index

NADPH: nicotinamide adenine dinucleotide phosphate (reduced form)

$\mathrm{NaOH}: \quad$ sodium hydroxide

NCBI: $\quad$ National Center for Biotechnology Information

$\mathrm{OH}^{-}$: hydroxide

PAR: photosynthetically-active radiation

$\mathrm{P}_{\mathrm{Net}}$ : $\quad$ net photosynthetic production

PCR: polymerase chain reaction

P/DOC: particulate and/or dissolved organic carbon

$\mathrm{P}_{\text {Gross: }}$ total photosynthetic production

pHi: $\quad$ internal $\mathrm{pH}$ inside the host cells

PIF: photosynthesis-inhibiting factor 
Abbreviations

$\mathrm{P}: \mathrm{R}$ ratio: $\quad$ ratio of maximum gross photosynthesis $(\mathrm{P})$ to symbiosis respiration (R) over 24 hours

$\mathrm{P}_{\mathrm{zNet}}$ : total net carbon fixed photosynthetically by zooxanthellae

$\mathrm{R}$ : $\quad 546 / 635 \mathrm{~nm}$ fluorescence intensity ratio

$\mathrm{R}_{\mathrm{a}}$ : host animal respiration

RNAi: $\quad$ RNA interference

ROI: $\quad$ region of interest

ROS: $\quad$ reactive oxygen species

RuPB: $\quad$ ribulose-bis-phosphate

Rz: $\quad$ zooxanthellar respiration

SOD: $\quad$ superoxide dismutase

$\mathrm{T}$ : $\quad$ percentage of total net carbon $\left(\mathrm{P}_{\mathrm{zNet}}\right)$ translocated to the animal

TCA: trichloroacetic acid

TEM: $\quad$ transmission electron microscope

UVR: ultraviolet radiation 


\section{Table of Contents}

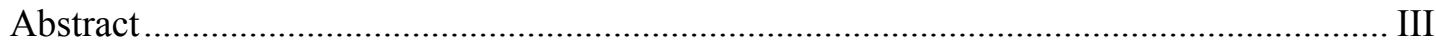

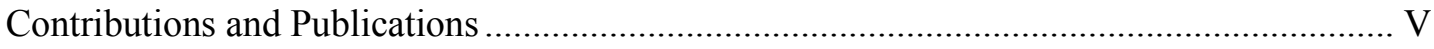

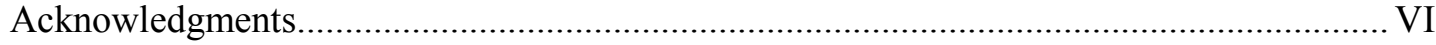

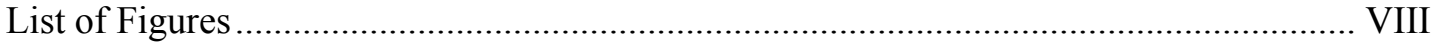

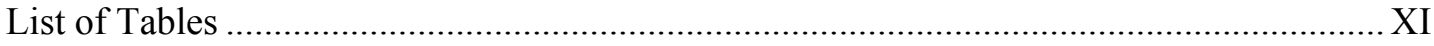

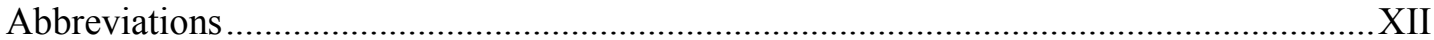

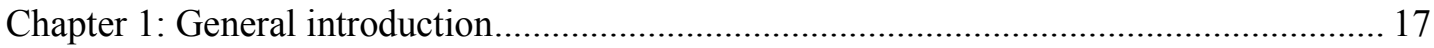

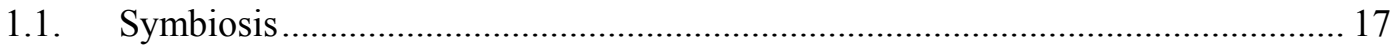

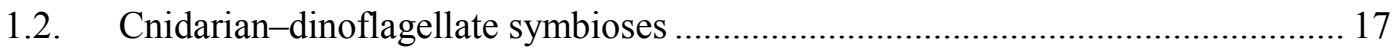

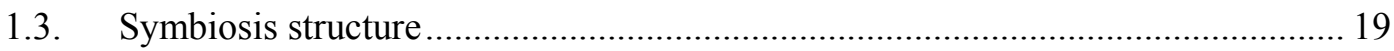

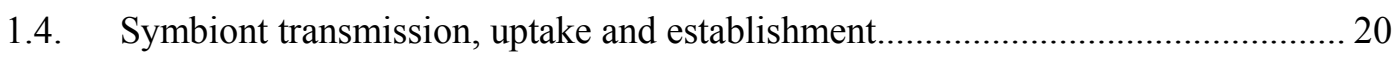

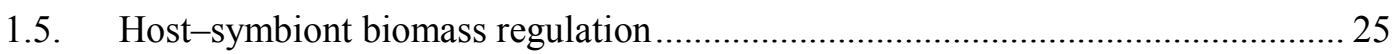

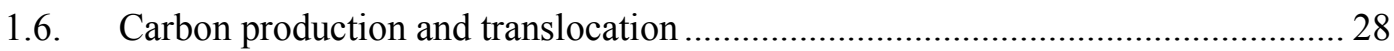

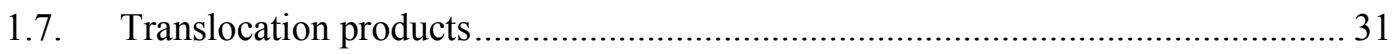

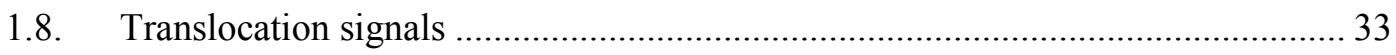

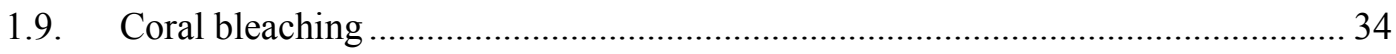

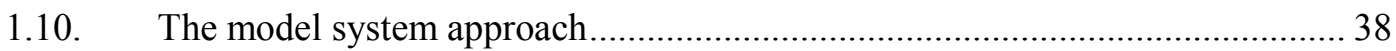

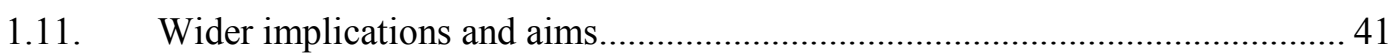

Chapter 2:

The influence of symbiont diversity on the photophysiological performance of novel

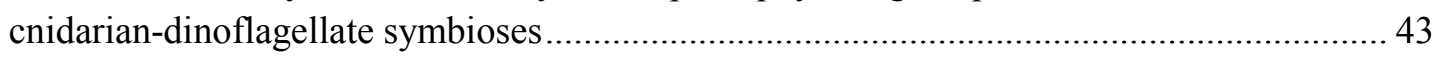

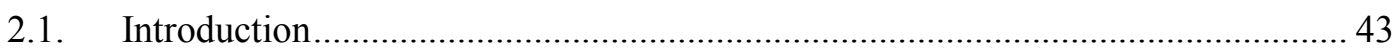

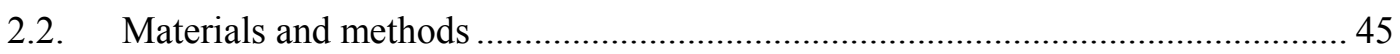

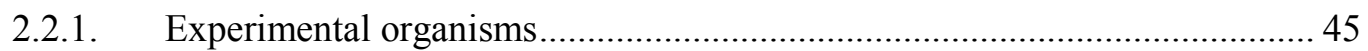

2.2.2. Infection of aposymbiotic A. pulchella with different Symbiodinium types .. 48

2.2.3. Symbiont growth rate, density, size and biomass .......................................... 48

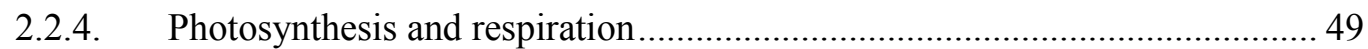

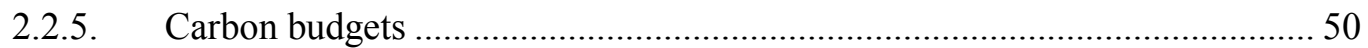

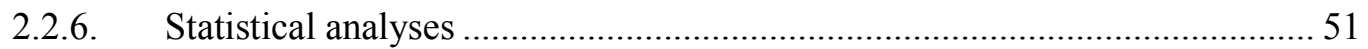

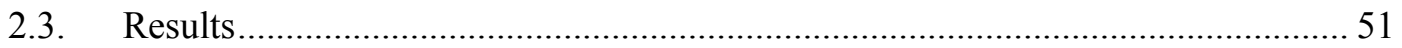

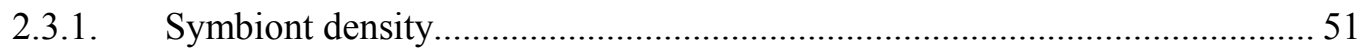

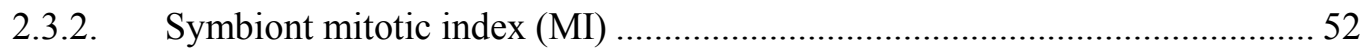

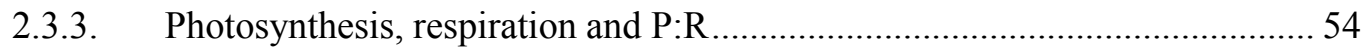

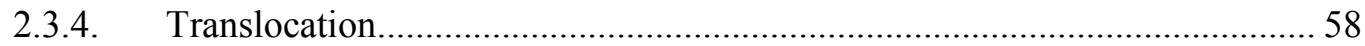


Table of Contents

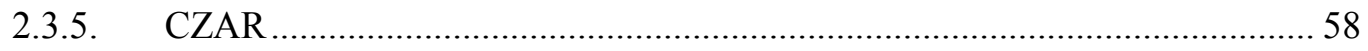

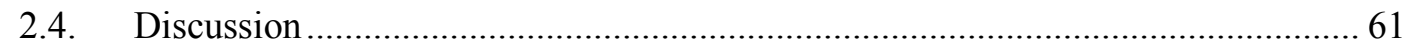

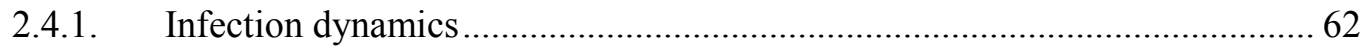

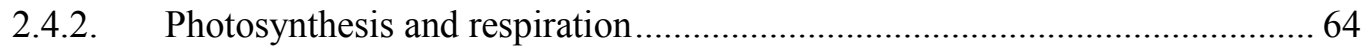

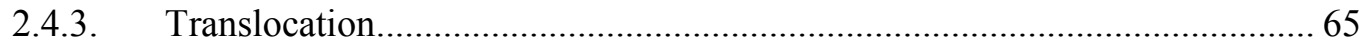

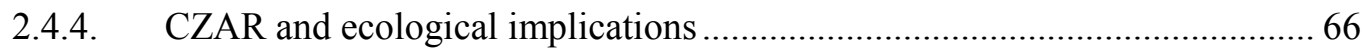

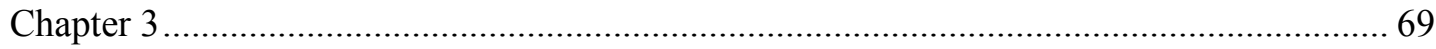

The influence of symbiont type on host cell $\mathrm{pH}$ and physiology of a novel cnidarian-algal

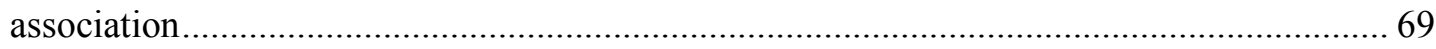

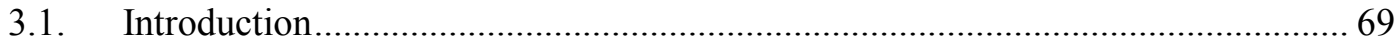

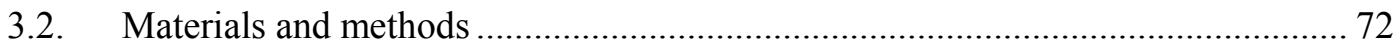

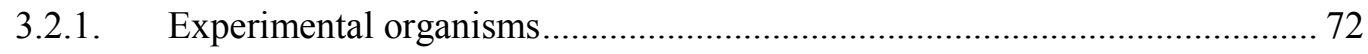

3.2.2. Infection of aposymbiotic A. pulchella with different Symbiodinium types .. 73

3.2.3. Symbiosome isolation and verification ....................................................... 74

3.2.4. $\mathrm{pH}$ manipulation and determination of photosynthetic rates ......................... 75

3.2.5. A. pulchella cell isolation and staining ....................................................... 77

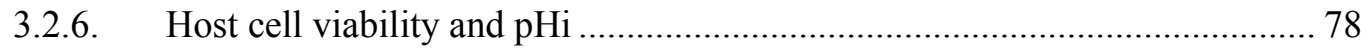

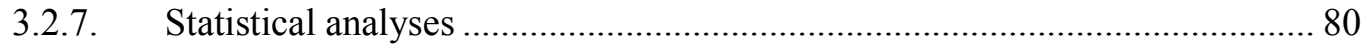

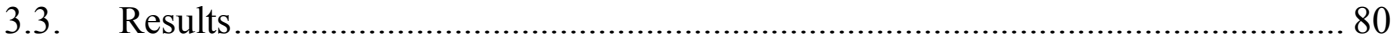

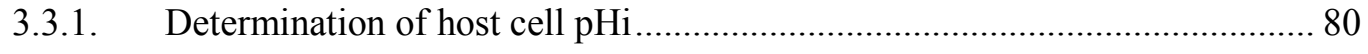

3.3.2. Effect of $\mathrm{pH}$ on gross photosynthetic and respiration rates ......................... 82

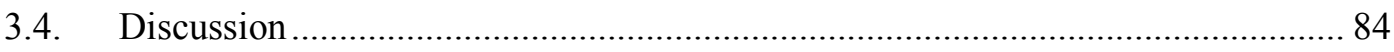

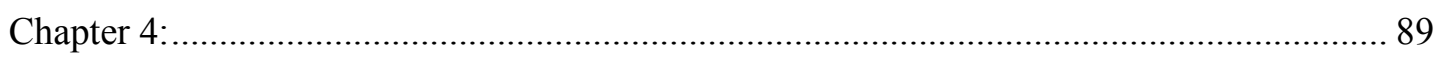

The influence of symbiont type on the glycerol and glucose pools in Aiptasia pulchella. 89

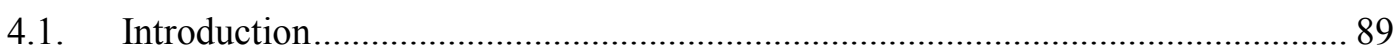

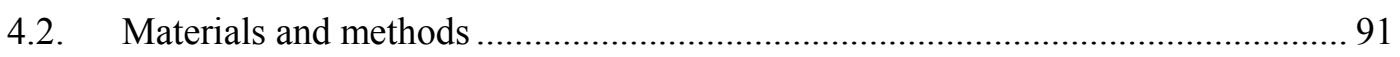

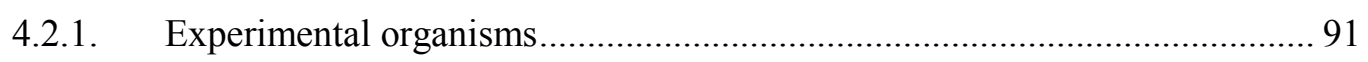

4.2.2. Infection of aposymbiotic A. pulchella with different Symbiodinium types .. 93

4.2.3. Levels of glycerol and glucose in host tissues ............................................ 93

4.2.4. Carbon fluxes in isolated Symbiodinium ....................................................... 94

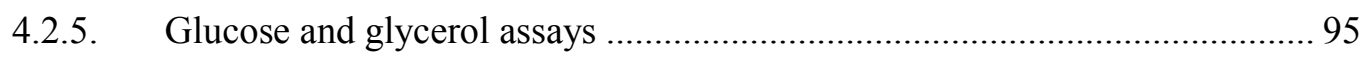

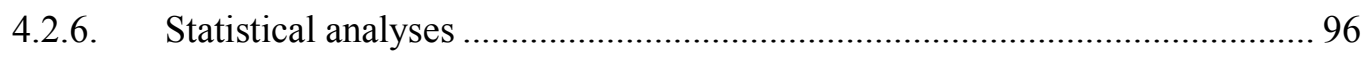

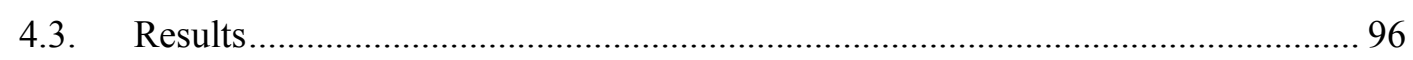

4.3.1. Glycerol and glucose levels in the host tissues ............................................. 96

4.3.2. Glycerol and glucose levels in the host tissue in the presence of $\mathrm{NaCN}$..... 103

4.3.3. Carbon fluxes in isolated Symbiodinium ...................................................... 107 


\section{Table of Contents}

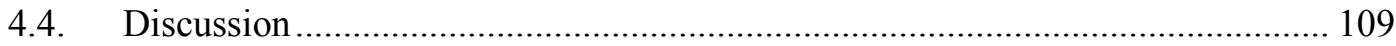

4.4.1. Glycerol and glucose levels in the host tissue............................................ 109

4.4.2. Carbon fluxes in isolated Symbiodinium ................................................... 112

4.4.3. The role of metabolite transfer in host-symbiont recognition and specificity 113

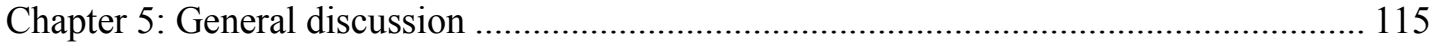

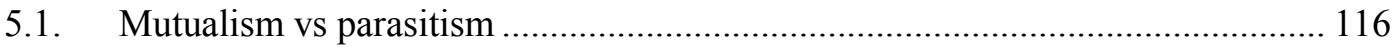

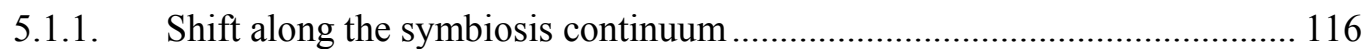

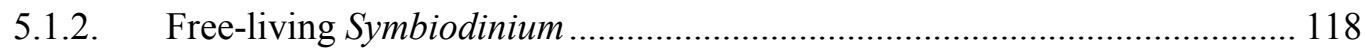

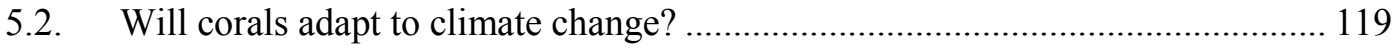

5.2.1. Symbiont type and the infection process ................................................ 119

5.2.2. The implications on the recovery of corals from bleaching events.............. 121

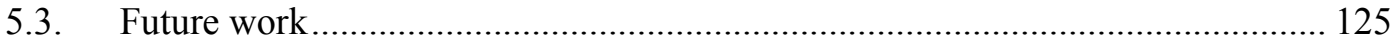

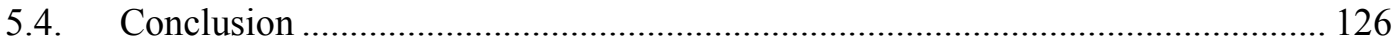

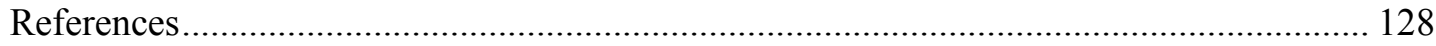

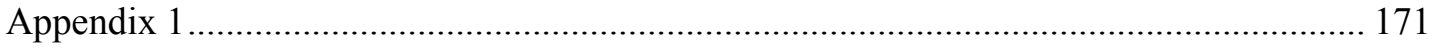

The influence of symbiont diversity on the photophysiological performance of novel cnidarian-dinoflagellate symbioses; complete carbon budget over entire 12 week period after A. pulchella infection with different Symbiodinium types ............................................... 171

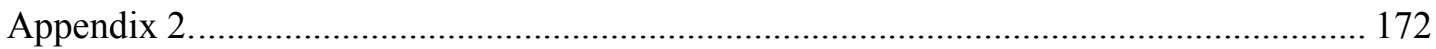

Measuring the host cell pHi in A. pulchella-Symbiodinium associations with different

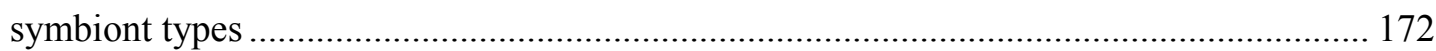

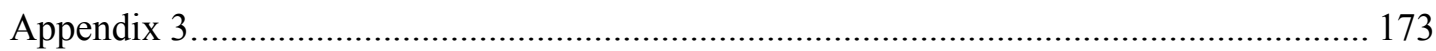

ITS2 sequences of Symbiodinium types used in this study .............................................. 173

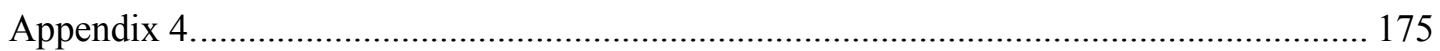

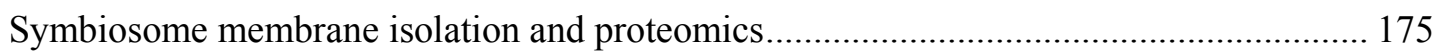

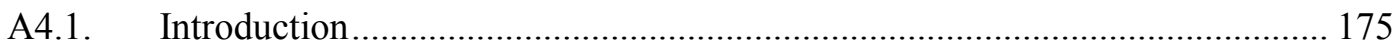

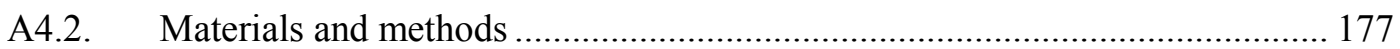

A4.2.1. Symbiosome membrane isolation .......................................................... 177

A4.2.2. Symbiosome and symbiosome membrane visualisation........................... 177

A4.2.3. Digestion of isolated membranes ................................................................ 178 


\section{Chapter 1: General introduction}

\subsection{Symbiosis}

Symbiotic associations were first formally described by the German mycologist Heinrich Anton de Bary, who coined the term "symbiosis". He defined symbiosis to be a relationship that is "constant, intimate and between dissimilar species" (de Bary, 1879). Symbiotic relationships include those associations in which one organism lives on another (ectosymbiosis, such as mistletoe), or where one partner lives inside the other (endosymbiosis, such as bacteria in humans or symbiotic algae in corals). Three broad types of symbiotic relationships have been recognized; mutualism, parasitism and commensalism (Smith, 1993). Mutualism is defined as a biological interaction between two different species where both partners derive a fitness benefit such as increased survival. Parasitism is a relationship whereby one partner derives a benefit but the symbiosis comes at a cost to the other partner. Commensalism, on the other hand, occurs when one partner benefits from the association and the other does not but is not harmed by it. However, many symbiotic associations are complex or poorly understood and do not fit neatly into a single category (Douglas, 1994). Therefore, it is better to visualise symbiosis as a continuum that spans from parasitism to mutualism (Figure 1.1; Dimijian, 2000) with a given relationship being able to shift gradually or abruptly along the continuum. Symbiosis can be further defined as facultative, where the relationship is beneficial but not essential to the survival of either partner; or obligate, where the relationship is essential to the survival of at least one partner (Vega et al., 2006).

\subsection{Cnidarian-dinoflagellate symbioses}

Cnidarian - dinoflagellate symbioses are widespread in the marine environment and consist of associations between anthozoans (e.g. corals, sea anemones, zoanthids and gorgonians) and dinoflagellates (commonly referred to as zooxanthellae) of the genus Symbiodinium (Trench, 1987; Furla et al., 2005). These symbioses are responsible for the formation of coral reefs which, in turn, maintain a rich 
Chapter 1

biodiversity in shallow tropical waters. They are of great ecological importance as coral reefs are vital in sustaining the coastal communities in these regions (Peterson and Lubchenco, 1997; Linden et al., 2002).

\title{
Pathogenicity $\longleftrightarrow$ Commensalism $\Longleftrightarrow$ Mutualism
}

\author{
Antagonism
}

Cooperation

Figure 1.1. A schematic diagram representing the continuum that exists between antagonistic and cooperative symbiotic relationships. Antagonistic relationships occur between hosts and parasites (or pathogens), cooperative relationships between mutualists, and mixed interactions between commensals (adapted from Dimijian, 2000).

The presence of the dinoflagellate symbionts contributes substantially to the productivity, survival and success of their hosts (Muscatine and Porter, 1977). A true mutualistic relationship exists between the host and symbiont, in that the symbiont receives inorganic nutrients from the host and provides the host with translocated photosynthetic products (Muscatine, 1990). In Cnidaria, the Symbiodinium cells are generally intracellular, located in cells of the endodermis (Schoenberg and Trench, 1980a; Douglas, 2003), and a perisymbiont membrane of animal origin separates them from the host cytoplasm (Wakefield and Kempf, 2001).

Freudenthal (1962) formally described the taxonomy, life cycle and morphology of these dinoflagellates and erected the genus Symbiodinium to encompass the symbiotic dinoflagellates associated with a phylogenetically diverse range of invertebrate hosts. It was first considered that symbiotic dinoflagellates were members of a single pandemic species, Symbiodinium microadriaticum (Freudenthal, 1962; Taylor, 1971 and 1974). In the mid-1970s, however, evidence began to accumulate that these dinoflagellates in fact exhibit a high degree of genetic diversity 
Chapter 1

and, at present, eleven formally described species plus multiple clades and sub-clades have been identified in the genus Symbiodinium (Baker, 1999; Coffroth and Santos 2005; Pochon and Gates, 2010).

Molecular studies using small subunit ribosomal DNA (ssrDNA) sequences have yielded nine distinct clades (A-I) of Symbiodinium (reviewed in Baker, 2003 and Coffroth and Santos, 2005; Pochon and Gates, 2010). Five of these clades (A-D, F) are known to occur in corals (Rowan and Powers, 1991; Baker, 1999; Carlos et al., 1999; LaJeunesse, 2001; Toller, 2001a, b; Ulstrup and van Oppen, 2003). With the advent of more sophisticated molecular tools, the biodiversity of the Symbiodinium genus has become increasingly well documented. Analyses of the internal transcribed spacer (ITS) region have revealed within-clade variation amongst Symbiodinium isolates (Hunter et al., 1997; LaJeunesse 2001, 2002; Santos et al. 2001; van Oppen et al. 2001; Diekmann et al. 2003; LaJeunesse et al., 2003, 2004a, b). Although numerous studies have assessed the biodiversity of both the coral host and symbiont partners individually (Vollmer and Palumbi, 2002; Coffroth and Santos, 2005), the diversity of host-symbiont combinations is still poorly understood (Baker, 2003; Goulet, 2006), and a better understanding of the mechanisms that confer recognition and specificity between the host and symbiont is necessary (Weis et al., 2008). Physiological differences (e.g. in thermal tolerance) have been detected between these Symboidinium ITS types (Iglesias-Prieto and Trench 1997; Kinzie et al., 2001; LaJeunesse et al., 2003). The presence of these differences has led to the suggestion that these symbiont types may affect their host's sensitivity to environmental conditions and/or that changes in environmental conditions may affect the distribution of symbiont types (van Oppen et al., 2005).

\subsection{Symbiosis structure}

There are numerous examples of symbioses in nature where one organism lives inside the cell cytoplasm of another i.e. endocytobiotic associations. These include: prokaryote-eukaryote associations (Jeon and Jeon, 1976; Lerouge et al., 1990; Regensburg-Tuink and Hooykaas, 1993; Udvardi and Day, 1997; Ferrari et al.,1999), 
Chapter 1

and eukaryote-eukaryote associations such as protozoan parasite-vertebrate symbioses (Sibley and Krahenbuhl, 1989; Hall et al., 1991; Russell et al., 1992; Sam-Yellowe, 1992; Collins et al., 1997; Forero et al., 1999) and microalgalinvertebrate symbioses (reviewed by Lee and Anderson, 1991; Trench, 1993). In most symbiotic associations, membranes surround the microsymbiont and separate it from the host cell cytoplasm. The terms "symbiosome" and "symbiosome membrane" were introduced by Roth et al. (1988) to describe the intracellular symbiotic compartment in which the symbiont resides and its surrounding membrane. In cnidarians, the symbiosome has been defined as the host-derived outer membrane, Symbiodinium algal cell and the space between the two, including a series of multilayered membranes (Wakefield et al., 2000; Wakefield and Kempf, 2001). The barrier between the host and symbiont may thus be more accurately described as a "membrane complex", with the outer host-derived membrane acting as the "definitive symbiosome membrane" (Figure 1.2; Kazandjian et al., 2008). The origin and nature of the multilayered membranes immediately surrounding the cell wall of the alga have been debated, with several studies suggesting that they are derived from the host (Tripodi and Santisi, 1982; Colley and Trench, 1983; Palincsar et al., 1988; Rands et al., 1993), while others have proposed an algal origin (Kevin et al., 1969; Taylor, 1971; Trench and Winsor, 1987; Wakefield et al., 1998, 2000). Using immunocytochemical methods, Wakefield and Kempf (2001) confirmed that the membranes originate from the algal symbiont and are held in place by the presence of a single host-derived membrane.

\subsection{Symbiont transmission, uptake and establishment}

There are two modes of transmission of symbionts from host to host: (1) From parent to offspring (vertical transmission) and (2) the acquisition of symbionts from the environment (horizontal transmission) (Trench, 1979; Douglas, 1994). Whereas vertical transmission occurs within the confines of the host's sexual cycle, horizontal transmission occurs via phagocytosis resulting in the formation of a symbiontcontaining host-derived vesicle (Smith and Douglas, 1987; Wakefield and Kempf, 2001). Free-living Symbiodinium cells are ingested probably via the host coelenteron. 
The cells are captured by the tentacles and transported by ciliated grooves on the tentacles and/or taken into the coelenteron with incoming seawater (Fitt and Trench, 1983). Inside the coelenteron, the algal cells are phagocytosed by the host gastrodermal cells and invested with a host-derived membrane (Kinzie, 1974; Fitt, 1984). Molecular mechanisms are likely to be involved in host-symbiont recognition. The identity of both the host and the symbiont partner has been shown to determine the success of the symbiosis (Coffroth et al., 2001; Weis et al., 2001; Belda-Baillie et al., 2002; Baker, 2003; Rodriguez-Lanetty et al., 2004, 2006a). Past studies have shown that cnidarian hosts are specific towards a unique symbiont type and that surface recognition plays a role in host-symbiont discrimination (Jolley and Smith, 1980; Schoenberg and Trench, 1980b; Colley and Trench, 1983; Logan et al., 2010; Bay et al., 2011). There is also biochemical and molecular evidence for the presence of glycoproteins on the cell surface of symbiotic dinoflagellates (Markell et al., 1992; Jimbo et al., 2000; Bay et al., 2011; Logan et al., 2010), while some studies have identified and characterised lectins on the surface of host cells that may be involved in a lock-and-key type recognition process (Fenton-Navarro et al., 2003; Koike et al., 2004; Jimbo et al., 2005; Wood-Charlson et al., 2006; Wood-Charlson and Weis, 2009; Kvennefors et al., 2010).

The onset of symbiosis in cnidarian/algal associations has been studied in a wide range of partnerships such as symbioses between: (i) the freshwater hydroid Hydra viridis and a chlorophyte (Muscatine et al., 1975, reviewed by Jolley and Smith 1980); (ii) the scyphozoan Cassiopeia xamachana and the dinoflagellate Symbiodinium microadriaticum (Colley and Trench 1983, 1985; Fitt and Trench 1983); and (iii) a variety of anthozoans and Symbiodinium spp. (e.g. Kinzie 1974; Schoenberg and Trench 1980b; Davy et al., 1997; Coffroth et al., 2001; BeldaBaillie et al., 2002; Rodriguez-Lanetty et al., 2003; Logan et al., 2010; RodriguezLanetty et al., 2006a). From these studies it was established that a winnowing process occurs in cnidarian/algal symbioses (Jolley and Smith, 1980; McAuley and Smith, 1982; Dunn and Weis, 2009). This process includes interaction between putative molecular signals on the surfaces of symbiont and hosts cells (Meints and Pardy, 1980; Markell et al., 1992; Lin et al., 2000; Koike et al., 2004), discrimination by the host early in infection between its own symbionts (homologous symbionts) and those from other host species (heterologous symbionts) (Pool, 1979; 
Jolley and Smith, 1980; Schoenberg and Trench, 1980b; Weis et al., 2001; BeldaBaillie et al., 2002; Rodriguez-Lanetty et al., 2004; Markell and Wood-Charlson, 2010), and a succession of different algal phylotypes later in host development (Rahat, 1991; Coffroth et al., 2001; Little et al., 2004; Gómez-Cabrera et al., 2008).

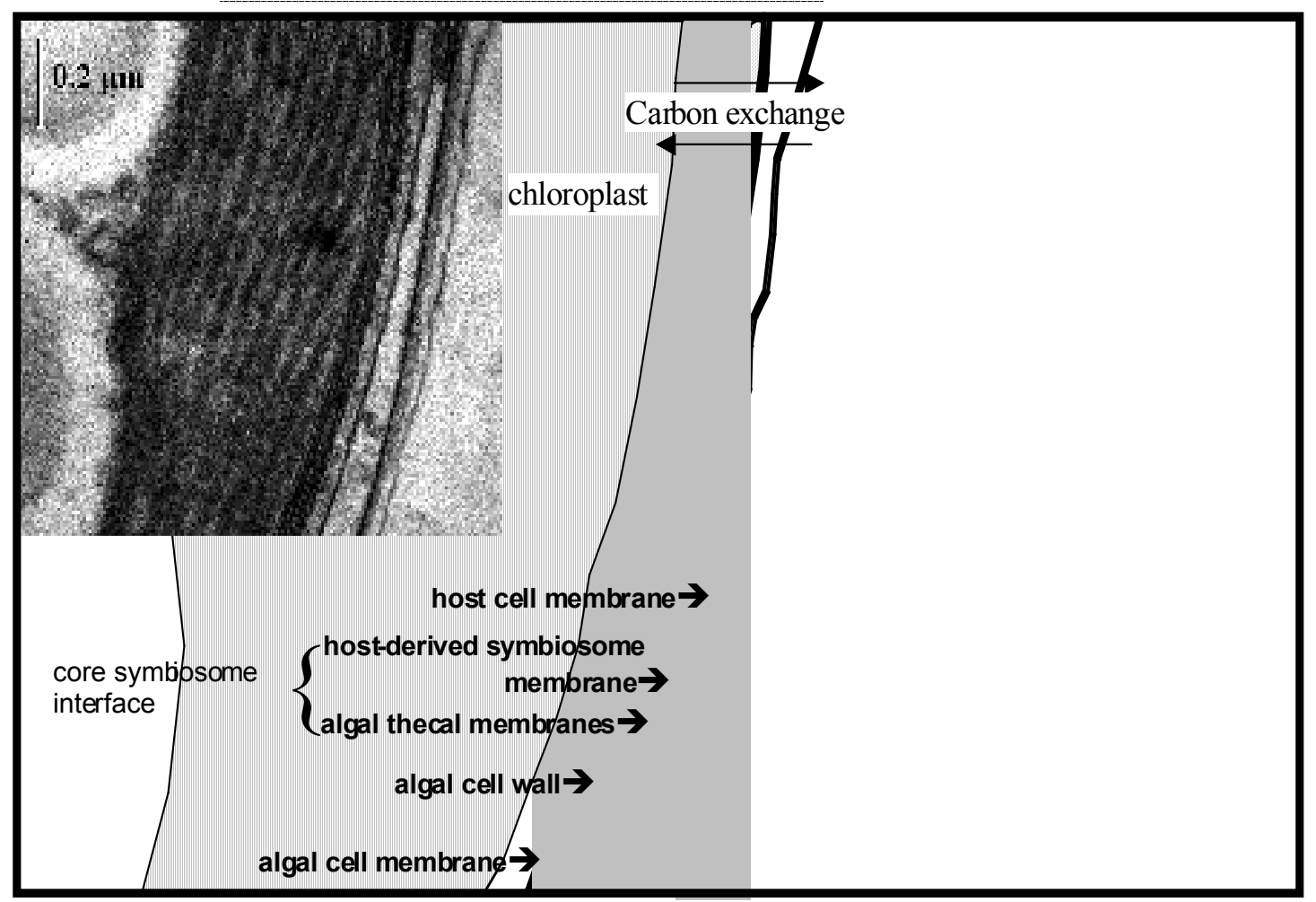

Figure 1.2. The symbiosome membrane complex - a transmission electron microscope (TEM) and graphic representation (obtained from Dr Rosanne Quinnell).

Little is known, however, about the cellular and molecular signals involved in maintaining the intracellular cnidarian - dinoflagellate symbiosis (Rodriguez-Lanetty et al., 2006b). How, for example, is the algal symbiont able to escape the host's immune response once inside the host cell, allowing for the successful persistence of a cnidarian-dinoflagellate symbiosis? It is possible that dinoflagellates may be utilizing or modifying the cnidarian's innate immune signalling pathways in order to avoid digestion of the phagosome in which they reside. Past studies of animal-algal symbioses have shown that, unlike phagosomes containing food particles, or dead or photosynthesis-impaired algae, the phagosomes containing live and functional algae do not show any signs of lysosomal fusion (Hohmann et al., 1982; Fitt and Trench, 
1983). This evidence indicates that live and functional zooxanthellae possess the ability to interfere with the normal path of phagosomal maturation so as to survive inside cnidarian cells (Chen et al., 2003, 2004, 2005; Hong et al., 2009). Studies of prokaryote-eukaryote symbioses have shown that, after entering the host cell, certain bacteria such as Mycobacterium tuberculosis (Armstrong and Hart, 1975), Chlamydia psittaci (Friis, 1972) and Legionella pneumophila (Horwitz, 1983) are able to avoid digestion by preventing fusion of the phagosome (vacuole containing the symbiont) with the host's lysosomes. The protozoan Toxoplasma gondii (Jones et al., 1972) and the alga Chlorella sp. (Hohman et al., 1982) are also capable of avoiding digestion in this way. Kim et al., (1994) identified a $96 \mathrm{kDa}$ protein and endosymbiont-derived lipopolysaccharides on the symbiosome membrane in a symbiosis between Amoeba proteus and a strain of gram-negative bacteria that were shown to be involved in the prevention of lysosome-symbiosome fusion.

Intracellular vesicular transport during endocytosis and exocytosis is thought to be mediated by Rab small GTP-binding proteins (peripheral membrane proteins). These proteins have been localized to distinct intracellular structures (Pfeffer, 2001; Zerial and McBride, 2001) and regulate fusion between various endocytic compartments by cycling between the GTP-bound, membrane-associated active state and the GDPbound, cytosolic inactive state (Pfeffer, 2001; Zerial and McBride, 2001). Rab 5 and Rab 7 have been shown to act coordinately and sequentially to facilitate the traffic of internalized materials to lysosomes. Rab 5 regulates fusion between clathrin-coated vesicles and early endosomes and between early endosomes (Bucci et al., 1992; Barbieri et al., 1994; Mukhopadhyay et al., 1997), whereas Rab 7 controls the fusion between early and late endosomes (Feng et al., 1995; Mohrmann and van der Sluijs, 1999), and between late endosomes and lysosomes (Papini et al., 1997; Vitelli et al., 1997; Mohrmann and van der Sluijs, 1999). In past studies, ApRab7 and ApRab5, Rab7 and Rab5 homologues of the sea anemone Aiptasia pulchella were cloned and it was established that the intracellular persistence of the zooxanthella-containing phagosomes involves specific algal-mediated interference with the expression of ApRab5 and ApRab7, which results in the selective retention of the former on (Chen et al., 2003) and exclusion of the latter from the organelles (Chen et al., 2004). Later, Chen et al. (2005) found that the homologue of Rab11 (ApRab11), a key endocytic recycling regulator, was excluded from phagosomes in which healthy zooxanthellae 
reside. More recently ApRab3 was identified as a new member of the Rab3 subfamily, associating with symbiosomes and accumulating on the maturing phagosomes in the A. pulchella digestive cells. It is has been shown to participate in the biosynthetic trafficking pathway, and symbiosome biogenesis has been demonstrated to involve interaction with ApRab3-positive vesicles (Hong et al., 2009). Thus, it appears that zooxanthellae must interfere with the normal recycling process required for efficient phagosome maturation, and thereby secure their intracellular persistence and consequently their endosymbiotic relationship with their cnidarian host (Chen et al., 2005).

The examination of either broad-scale patterns of RNA or protein expression in symbiotic cnidarians or the identification of specific genes that play a role in signal transmission between host and symbiont are still in their early stages (RodriguezLanetty et al., 2006b; Bay et al., 2009, Voolstra et al., 2009, Ganot et al., 2011). A number of studies have, however, attempted to compare the gene expression patterns of symbiotic versus aposymbiotic individuals to determine which genes may play a role in governing the symbiotic association. Using two-dimensional proteomic analysis to compare symbiotic and naturally occurring aposymbiotic individuals, Weis and Levine (1996) revealed a number of proteins that were up- or downregulated in the symbiotic state of the US Pacific coast temperate anemone Anthopleura elegantissima. One of the strongly expressed symbiotic proteins, sym32, was later identified as belonging to the Fasciclin I protein family which includes proteins that function in cell-cell interactions or cell adhesion in other organisms (Reynolds et al., 2000). Further immunocytochemistry and immunoblot studies by Schwarz and Weis (2003), using an anti-sym32 antibody, found a putative homologue in the symbionts. Their study revealed that sym32 is relocated from gastrodermal vesicles (where it was found in aposymbiotic anemones) to the symbiosome membrane when symbionts are phagocytosed by host cells. Thus the cnidarian host and its symbiont may utilise heterophilic Fasciclin I interactions as a means of inter-partner signalling (Schwarz and Weis, 2003; Rodriguez et al., 2006b). However, the studies by Barneah et al. (2006) and deBoer et al. (2007) revealed virtually no changes in the host proteome before and after infection with symbionts. This suggests that during the first days and weeks of symbiosis, translational and 
Chapter 1

post-translational changes specific to the symbiotic state may not be occurring in the host (Barneah et al., 2006).

The symbiosome membrane complex acts as a barrier between the host cell cytoplasm and the symbiont thus an understanding of the communicative processes between the algal symbiont and its cnidarian partner, which are no doubt crucial to the nutrition, stability and persistence of the symbiotic relationship, is necessary. It is essential to characterise the properties of the symbiosome membrane as the transport properties of this membrane govern what passes between the host and symbiont. Kazandjian et al. (2008) isolated the symbiosome membrane from the zoanthid, Zoanthus robustus, using a combination of mechanical disruption and sucrose gradient centrifugation. Using confocal microscopy and TEM they confirmed that the symbiosome interface surrounds the alga and also established that it is multi-layered, robust and displays a level of heterogeneity in terms of thickness. Since then Peng et al. (2010) undertook a proteomic analysis of the membrane and were able to determine some of the major membrane proteins involved in membrane formation, signalling, molecular transport between the symbiont and host, symbiont recognition, photosynthesis, and host and symbiont cell cycle regulation. More studies of this kind aimed at examining the membrane composition and membrane trafficking using fluorescent markers are necessary to gain an understanding of the symbiosomemembrane modification that occurs during symbiosis (Davy et al., 2012).

\subsection{Host-symbiont biomass regulation}

In order for an association to persist in the long-term, both host cell growth and symbiont population proliferation has to be well regulates (Davy et al., 2012). As the number of symbionts present inside the host increases so the host must accommodate them by growing additional cells or regulating their numbers (Muscatine et al., 1998). The potential mechanisms that have been suggested to regulate algal cell numbers in algal-invertebrate symbioses can be divided into two groups; pre-mitotic mechanisms and post-mitotic mechanisms. Pre-mitotic mechanisms that regulate symbiont numbers may include density-dependent negative feedback by limiting 
Chapter 1

nutrients or space (Jones and Yellowlees, 1997), host-induced release of photosynthate from the symbionts (Gates et al., 1995), and host manipulation of the symbiont cell cycle (Smith and Muscatine, 1999). In the green Hydra symbiosis has been shown to be regulated by restrictions on the cell cycle that limit progression through interphase and into the M phase (Douglas and Smith, 1984; McAuley, 1985a and b; McAuley and Muscatine, 1986; but see Pardy, 1981). Past studies have yielded four possible mechanisms for the control of the cell cycle in Hydra:

(1) $\mathrm{pH}$ stimulated release of photosynthate (maltose) to the host leading to a reduction in symbiont growth (Douglas and Smith, 1984; McAuley, 1985c) The internal $\mathrm{pH}$ inside the host cells (pHi) is known to play a role in the regulation of the cell growth cycle in some organisms and could provide a mechanism by which host cnidarians regulate cell division by their symbionts (Davy et al. 2012). Chlorella cells for example, have been shown to release maltose in response to acidifiacation (Cernichiari et al., 1969; Mews, 1980; Muscatine et al., 1967) and those Chlorella cells that release large amounts of maltose have been shown not to grow at low $\mathrm{pH}$ in vitro (Douglas and Smith, 1984). These finding suggests that small changes in the $\mathrm{pH}$ of the symbiosome space (space between the symbiont and host), could affect how much photosynthate is translocated from symbiont to host. This in turn could directly or indirectly influence the cell cycle. However, evidence for this is yet to be demonstrated in hospite (Davy et al., 2012).

(2) Host-regulated supply of a metabolite(s) (McAuley, 1982, 1985a, 1985b; McAuley and Muscatine, 1986). Studies by McAuley (1985a, b) and McAuley and Darrah (1990) suggested that restriction by the host to a pool or pools of metabolites derived from host digestion, may limit symbiont division.

(3) Regulation by the host of inorganic nutrient supply to the symbiotic algae (Muscatine and Pool, 1979; McAuley, 1982, 1985c; Blank and Muscatine, 1987). Studies have shown that by limiting the supply of certain inorganic nutrients to the symbiont, the host is able to restrict the growth of the 
Chapter 1

symbiont population. Rees (1986), for example, demonstrated that Hydra has the ability to assimilate and withhold ammonium from its symbiont.

(4) Algal production of a density-dependent inhibitor (Muscatine and Pool, 1979; McAuley, 1982). Experimental support for this mechanism is still lacking.

Premitotic mechanisms in cnidarian-dinoflagellate associations are thought to operate in a similar manner especially with respect to the restriction of the supply of inorganic nutrients to the symbiont and loss of photosynthetic carbon to the host (Davy et al., 2012), however the studies of these mechanisms in these symbioses is limited. In the Aiptasia pulchella, nutrient supply has been shown to influence the biomass, composition and physiology of the algal symbionts however, it did not limit symbiont growth (Smith and Muscatine, 1999). Instead it appears that the cellular growth of the host and the progression of the symbiont through the cell cycle is linked (Smith and Muscatine, 1999). A similar conclusion was reached by Fitt and Cook (2001) for the hydroid, Myrionema amboinense. Light has also been found to be important for the division of symbiotic dinoflagellates (Fitt, 2000). In a cultured Symbiodinium sp. belonging to clade B from the coral Euphyllia glabrescens, the light/dark period was demonstrated to encourage progression from the G1 phase to the S phase, and then to the G2 and M phases (Wang et al., 2008). Wang et al. (2008) found that a green fluorescent protein in the tissues of this coral dissipates blue light but, when added to a Symbiodinium culture, it inhibits progression through the G1 phase to $\mathrm{S}$ phase to $\mathrm{G} 2 / \mathrm{M}$ phase but not cytokinesis. This suggests that the host may be able to control symbiont growth through the manipulation of the light regimen (Davy et al., 2012).

Post-mitotic mechanisms involve the removal of existing zooxanthellae by degradation and digestion of symbionts (Titlynanov et al., 1996) or the expulsion of intact symbionts (Hoegh-Guldberg et al., 1987). In Hydra there is good evidence that the expulsion of algal cells plays an important role in symbiont population control (Fishman et al., 2008). In cnidarian-dinoflagellate associations, expulsion (Reimer, 1971; Trench, 1974; Steele, 1975 and 1977; Hoegh-Guldberg et al., 1987; HoeghGuldberg and Smith, 1989; Muscatine et al., 1991; Stimson and Kinzie, 1991; Jones 
Chapter 1

and Yellowlees, 1997) also appears to be employed with there being less evidence for symbiont cell degradation. Smith and Muscatine (1999) proposed that expulsion is associated with host cell turnover (Gates et al., 1992; Jones and Yellowlees, 1997). Baghdasarian and Muscatine (2000) later concluded that expulsion is one of the primary regulators of the symbiont density in some cnidarian-dinoflagellate symbioses. Evidence for this was demonstrated in the anemone, Anthopleura elegantissima, in which the expulsion of algal cells can exceed the daily growth of the symbionts at irradiances of less than full sunlight (McCloskey et al., 1996). In the temperate Atlantic coral Astrangia poculata, relatively high rates of expulsion allow the coral to sometimes persist in a stable, near-nonsymbiotic state (Dimond and Carrington, 2008). The cellular mechanisms behind the expulsion mechanism however, are still largely unknown (Davy et al., 2012). Dead symbionts cells and cellular debris have been observed in the tissues of reef corals (Titlyanov et al., 1996, 2004 and 2006), other anthozoans (Trench, 1974; Steele and Goreau, 1977) as well as the hydroid Myrionema amboinense (Fitt and Cook, 1990; Fitt, 2000). Degraded symbionts and cellular debris have also been identified in pellets extruded by various tropical sea anemones (Steele, 1977; Steele and Goreau, 1977). However, the mechanisms of symbiont degradation have been examined only in the context of stress and bleaching and not as part of a homeostatic mechanism (Davy et al., 2012).

\subsection{Carbon production and translocation}

The carbon fixed by the zooxanthellae (probably by the C3 pathway) is used to support their respiration and growth (Streamer et al., 1993). A portion of this carbon is translocated to the host (Muscatine et al., 1983; Davies, 1984) in the form of glucose, glycerol (Muscatine, 1967; Trench, 1974; Schmitz and Kremer, 1977) and lipid (Kellogg and Patton, 1983; Patton et al., 1983; Muscatine et al., 1984; Peng et al., 2011). The translocated carbon that is made available to the host is used for host respiration, growth and reproduction or is released from the association (Crossland et al., 1980a, b; Muscatine et al., 1981; Edmunds and Davies, 1986; Rinkevich, 1989; McCloskey et al., 1994). 
Muscatine and Hand (1958) were the first to demonstrate photosynthate translocation in the symbiotic, temperate sea anemone Anthopleura elegantissima by using autoradiography to track radiolabeled organic carbon in the anemone's tissues. An attempt to measure the release of photosynthetic products from Symbiodinium cells was later made by Muscatine (1967). He incubated freshly isolated Symbiodinium cells in homogenized host tissue and found that the zooxanthellae released significant amounts of soluble photosynthate to the host homogenate. In order to quantify the amount of photosynthate released by the zooxanthellae, Trench (1971a-c) ran a series of experiments using ${ }^{14} \mathrm{C}$ to measure the release of photosynthetic products in Anthopleura elegantissima. He concluded that approximately $40-50 \%$ of the fixed ${ }^{14} \mathrm{C}$ was made available to the host. Figure 1.3 illustrates an example of the daily budget for photosynthetically fixed carbon in a symbiotic cnidarian.

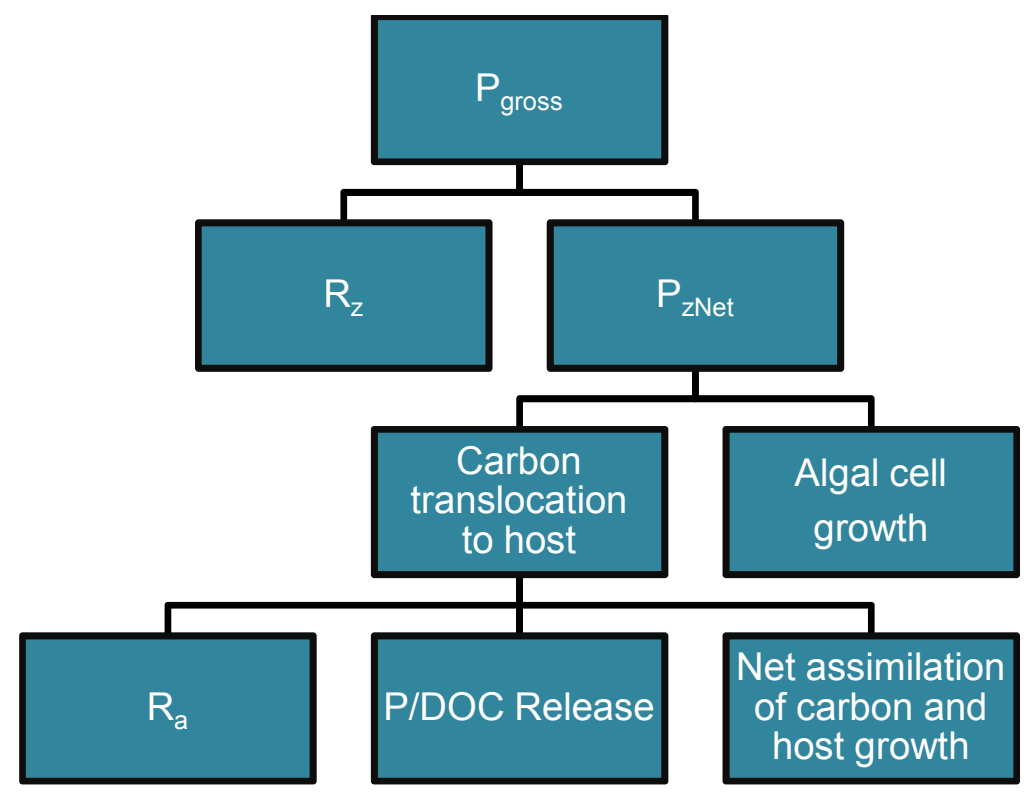

Figure 1.3. Representation of the daily budget for photosynthetically fixed carbon in a symbiotic cnidarian. $\mathrm{P}_{\text {gross }}$ is the total photosynthetic production, $\mathrm{P}_{\mathrm{zNet}}$ is net photosynthetic production after allowing for zooxanthellar respiration $\left(\mathrm{R}_{\mathrm{z}}\right), \mathrm{R}_{\mathrm{a}}$ is host animal respiration and $\mathrm{P} / \mathrm{DOC}$ is particulate and/or dissolved organic carbon released by the host (reproduced from Muscatine et al., 1983).

The ratio of maximum gross photosynthesis $(\mathrm{P})$ to symbiosis respiration $(\mathrm{R})$ over 24 hours is a useful measure of the autotrophic potential of a symbiotic association 
Chapter 1

(Davies, 1977; Leletkin, 2000). A P:R value that exceeds 1 assumes that the symbiosis is autotrophic with respect to carbon and that there is a surplus of photosynthetically-fixed carbon available. A P:R value of less than 1 , on the other hand, indicates a polytrophic symbiosis which can only be partially fulfilled photosynthetically and relies on an external carbon source (Muscatine et al., 1981).

Most cnidarian-dinoflagellate symbioses from tropical regions have been found to be autotrophic with respect to carbon (Muscatine et al., 1984). Temperate symbioses, on the other hand, generally cannot meet the demands of respiration through photosynthesis alone and as a result must acquire additional carbon from heterotrophic sources (Muller-Parker and Davy, 2001). The external carbon is sourced through predation on zooplankton, uptake of particulate and dissolved organic material from the environment (Lewis and Price, 1975), mucus feeding, and feeding on micro-organisms associated with detritus and mucus (Muscatine et al., 1984). However, the external uptake of carbon by a cnidarian host, as a means of compensating for insufficient carbon translocation by its symbionts, is most likely to be species dependent (Anthony and Fabricius, 2000)

In 1981, Muscatine et al. further developed the energy budget models and derived an equation to calculate the fractional contribution of translocated zooxanthellar carbon to the host's daily respiratory carbon requirements (CZAR):

$$
\mathrm{CZAR}=\frac{\mathrm{P}_{\mathrm{zNet}} * \mathrm{~T}}{\mathrm{R}_{\mathrm{a}}}
$$

\section{Equation 1}

Where $\mathrm{P}_{\mathrm{zNet}}$ is the total net carbon fixed photosynthetically by the zooxanthellae, $\mathrm{T}$ is the percentage of $\mathrm{P}_{\mathrm{zNet}}$ translocated to the animal, and $\mathrm{R}_{\mathrm{a}}$ is the carbon respired daily by the animal. The major components used to estimate the CZAR are photosynthesis, respiration, algal reproduction (cytokinesis), and algal translocation (Verde and McCloskey, 1996a). This equation has been applied to a number of symbiotic associations (e.g. Muscatine et al., 1984; Edmunds and Davies, 1986 and 1989, Davy 
Chapter 1

et al., 1996; Verde and McCloskey, 2002), suggesting that up to 99\% of carbon fixed in photosynthesis is translocated to the host (Davy et al., 1996).

\subsection{Translocation products}

There is still much uncertainty about the compounds that are translocated to the host (see review by Davy et al., 2012). Muscatine (1967) was the first to isolate zooxanthellae from corals and giant clams and identify both the intracellular and released products by two dimensional (2-D) radiochromatography after incubation of the algal cells in host tissue homogenate. The study revealed that glycerol (up to $90 \%$ of the total carbon released) was the major product released by these isolated zooxanthellae, with glucose and a ninhydrin-positive compounds also being released. Since then, many other studies have applied this same method to cnidariandinoflagellate associations and have also found glycerol to be the main compound released, with other metabolites such as glucose, the amino acid alanine, and organic acids such as fumarate, succinate, malate, citrate, and glycolate being released in smaller amounts (Von Holt and Von Holt, 1968; Trench 1971a, b and 1974; Sutton and Hoegh-Guldberg, 1990). Patton and Burris (1983) and Kellog and Patton (1983) also identified fat droplets being released from dinoflagellate cells isolated from the coral Stylophora pistillata and the sea anemone Condylactis gigantean, indicating that lipids may also be translocated.

However, since isolated zooxanthellae do not behave in the same manner as when they are inside the host (Roberts et al., 2001; Davy et al., 2012), many studies have also attempted to identify translocated compounds in hospite. This has proved to be very challenging, both due to the intracellular nature of zooxanthellae when in symbiosis, and the potentially rapid catabolism of translocated compounds in the host tissue. Nevertheless, a number of indirect approaches have been employed and have included, but are not limited to, studies that track the incorporation of ${ }^{14} \mathrm{C}$ labelled compounds into the host tissues (Muscatine and Cernichiari, 1969; Trench, 1971c; Blanquet et al., 1979; Battey and Patton, 1984 and 1987), microscopic analyses of cnidarian tissues (Harland et al., 1991, Gautret et al., 1997; Wang and Douglas., 1999; Luo et al., 2009; Chen et al., 2012), and the proteomic and 
ultrastructural analysis of "lipid bodies" isolated from symbiotic coral cells (Peng et al., 2011). More recently the entire host genome of the coral Acropora digitifera was analysed providing more information about the types of compounds translocated from symbiont to host (Shinzato et al., 2011). The outcome of this research again indicated that the major components of the translocated material are glycerol, amino acids and various lipids.

An alternative approach involved the addition of potential translocated compounds to the medium surrounding a ${ }^{14} \mathrm{C}$-labelled symbiosis and the saturation of any uptake sites (Lewis and Smith, 1971). This stimulated the release of ${ }^{14} \mathrm{C}$-labelled compounds into the surrounding medium and again supported the translocation of glycerol, glucose and alanine; lipids were not considered. Whitehead and Douglas (2003) used a similar approach to identify the compound translocated in the anemone Anemonia viridis, by comparing ${ }^{14} \mathrm{C}$-labelled compounds in the host tissue between treatments treated with $\left[{ }^{14} \mathrm{C}\right]$ bicarbonate under photosynthesizing conditions and when exogenous ${ }^{14} \mathrm{C}$-labelled substrates were applied in the dark. They assumed that when the labelling patterns produced by both treatments were similar, then the exogenous compounds matched (or were similar to) the translocated compounds. This study identified glucose, succinate and fumarate as potential translocated metabolites but, contrary to previous studies, it found no evidence for glycerol translocation. Indeed, there is debate as to whether glycerol is translocated at all, with other studies (of the giant clam-Symbiodinium symbiosis) also suggesting that it does not form part of the translocate (Rees et al., 1993; Ishikura et al., 1999). More recently Burriesci et al. (2012) labled intact Aiptasia sp. in the light with ${ }^{13} \mathrm{C}$-bicarbonate and, after homogenization and separation of animal and algal fractions, found labelled glucose in the animal fraction. However, they found no significant labelling of glycerol in the animal tissues thus supporting previous studies that indicated that glycerol does not form a major part of the translocate. Discrepancies exist between the compounds translocated in different symbioses as well between the compounds released by isolated Symbiodinium cells and those in hospite (Davy et al., 2012). Much more research is therefore necessary for us to conclusively state which compounds are translocated, and what qualitative and quantitative differences exist between different symbioses. 
Chapter 1

\subsection{Translocation signals}

Information about what stimulates and controls the translocation of photosynthetically fixed carbon from symbiont to host is still, to a large extent, lacking. A few hypotheses have, however, been proposed. One suggestion is that, by actively "blocking" symbiont mitosis or restricting the supply of nutrients to the symbiont, the host is able to limit the growth of the symbionts and in so doing 'free up' more carbon for translocation (Muscatine et al., 1983; Falkowski et al., 1984). A range of studies have also demonstrated that isolated zooxanthellae can be stimulated to release a substantial portion of their photosynthetically fixed carbon when incubated in host tissue homogenate (Trench, 1971c; Muscatine et al., 1972; Muscatine et al., 1984; Hinde, 1988; Sutton and Hoegh-Guldberg, 1990; Grant et al., 1997; Davy and Cook, 2001a), suggesting that the host produces some sort of signal that initiates this release. The signal molecule(s) that is thought to mediate this process has been termed the "host release factor" or HRF. The identity of the HRF is still unknown despite attempts to characterize it. Gates et al. (1995) proposed that it consists of a range of free amino acids (FAAs), though this idea has been disputed, with other studies suggesting that unknown small host compounds $(<100 \mathrm{Da})$ act as HRFs (Withers et al., 1998; Cook and Davy, 2001). Continuing research by Grant and co-workers (Ritchie et al., 1993; Grant et al., 1997, 1998, 2001, 2006a, b) has identified two water-soluble signalling molecules of $1000 \mathrm{kDa}$ or less, one that stimulates photosynthate release (HRF) and another that partially inhibits photosynthesis, termed the "photosynthesis-inhibiting factor" (PIF). They demonstrated that HRF from the coral, Plesiastrea versipora, stimulates translocation by either increasing glycerol synthesis or diverting it away from the symbiont's triglyceride stores (Grant et al., 1997). The limitation of HRF studies, however, is that they do not replicate the in hospite environment and thus the HRF factor may simply be an experimental artifact (Davy and Cook 2001a, b; see review by Davy et al., 2012). 
Chapter 1

\subsection{Coral bleaching}

Coral bleaching refers to the whitening of corals due to the mass expulsion of symbiotic algae and/or the loss of photosynthetic pigments within individual zooxanthellae (see Figure 1.4). Photosynthetic pigments are contained within the chloroplasts of the symbionts and thus corals that have lost their symbionts appear transparent with their white calcium carbonate skeletons visible through the tissue layers (Glynn, 1991: Baker, 2003; Lesser and Farrel, 2004). In recent years there has been an increase in the frequency and severity of episodes of coral bleaching resulting in degradation and mortality of coral reefs on a global scale (Baker, 2003; Lesser and Farrel, 2004; Obura, 2005; Hoegh-Guldberg et al., 2007; HoeghGuldberg and Bruno, 2010). A variety of stressors are thought to be responsible for coral bleaching, the most important being increased sea temperature, solar radiation, and a combination of both factors (Brown, 1997; Lesser and Farrell, 2004). Because symbioses with zooxanthellae are commonly found in habitats at $1-2{ }^{\circ} \mathrm{C}$ below the temperature which triggers breakdown of the symbiosis, they are extremely sensitive to increases in temperature (Douglas, 2003). A number of cellular mechanisms have been proposed for coral bleaching. These include: the in situ degradation of zooxanthellae; the loss of algae into the coelenteron and hence ambient seawater; and the release of intact endodermal cells containing intracellular zooxanthellae (Gates et al., 1992; Brown, 1997; Weis, 2008). It is likely that the pathway of zooxanthellar degradation may differ depending on the intensity and duration of stress and also the host species (Mise and Hidaka, 2003). Smith et al. (2005) suggested that hydrogen peroxide generated within zooxanthellae may have a signalling role in triggering the mechanisms that result in expulsion of the zooxanthellae from the coral.

Photosynthetically-active radiation (PAR) and/or ultraviolet radiation (UVR) may act in concert with elevated temperature to elicit a bleaching response (Brown, 1997; Warner et al., 1999; Lesser and Farrel, 2004). The role that UVR may play in increasing future levels of coral bleaching is one of the main areas of concern. The amount of UVR that submerged corals are exposed to is related to stratospheric ozone levels, changing weather patterns and the attenuation of UVR in the water column (Brown, 1997). Therefore, increases in ozone depletion, global warming and 
anthropogenic influences that affect both particulate loads and dissolved organic carbon (DOC) in oceans, all contribute to coral bleaching (Brown, 1997). Lesser and Farrel (2004) showed that corals exposed to supra-optimal solar irradiance and elevated seawater temperatures exhibit greater damage to the photosynthetic apparatus. An increase in water temperature results in a decrease in the photosynthetic activity of the symbiosis, most probably associated with a decrease in the ability of Symbiodinium spp. to process captured light. This causes dysfunction in photosystem II. Damage to the chloroplast and photosynthetic apparatus can occur in at least three inter-related ways that act together to start the bleaching cascade (Weis, 2008): (1) Damage to the D1 protein can occur during elevated temperature in Symbiodinium, the D1 (Warner et al., 1999) and heat may also damage the protein's repair mechanism itself (Takahashi et al., 2004). D1 damage results in a backup in excitation energy and the dysfunction of photosystem II (Warner et al., 1999). (2) Both heat and light have been shown to negatively impact the dark reaction of photosynthesis such that carbon fixation declines (Jones et al., 1998). This is because the enzyme responsible for primary carboxylation, ribulose bisphospate carboxylase oxygenase (Rubisco), may be damaged (Lesser, 1996) resulting in reduced consumption of adenosine triphosphate (ATP) and nicotinamide adenine dinucleotide phosphate (NADPH) coming from the light reactions. Excitation energy can back-up due to this leading to the dysfunction of photosystem II (Jones et al., 1998; Venn et al., 2008). (3) The thylakoid membranes can also be damaged by elevated temperature and light (Tchernov et al., 2004) causing the energetic uncoupling of electron transport in both photosystems. This inhibits the production of ATP and NADPH. All three of these mechanisms can lead to the build up of electrons which is thought to ultimately lead to the generation of multiple reactive oxygen species (ROS) in the symbiont. The fluxes of ROS overwhelm host and algal antioxidant defense systems, resulting in damage to both the light (photosystem II) and dark reactions (carbon fixation) in photosynthesis (Lesser and Farrel, 2004; Weis, 2008). Under high temperature and light conditions, antioxidants such as superoxide dismutase (SOD) and ascorbate peroxidase (APX) that quench and convert superoxides into less harmful molecules are saturated, resulting in damage to cellular components (Hoegh-Guldberg and Smith 1989; Glynn and D'Croz 1990; Lesser et al., 1990; Iglesias-Prieto et al., 1992; Lesser, 2004; Fitt and Warner, 1995). As a result, ROS are able to further damage photosynthetic membranes, as described 
Chapter 1

above, in an escalating positive feedback loop (Lesser, 2006; Weis, 2008). It is also able to diffuse into the host tissue where the damage continues and ultimately leads to bleaching (Weis, 2008).

There is evidence that both the host and symbiont have developed protective mechanisms to tolerate stress. These mechanisms include an increased expression of heat shock proteins and antioxidant enzymes as well as the production of protective pigments (Coles and Brown, 2003; Douglas, 2003; Venn et al., 2008; Weis, 2008). Tolerance to stress appears to vary within a population or between species of either or both hosts and symbionts (Coles and Brown, 2003; Douglas, 2003; Venn et al., 2008). Single host species associated with different algal genotypes have been shown to vary in bleaching susceptibility (Rowan et al., 1997; Perez et al., 2001). Symbiodinium communities have also been shown to vary in their photobiological characteristics and different coral-Symbiodinium associations may display different photophysiological responses (Robinson and Warner, 2006; Hennige et al., 2009). Photoacclimation refers to the phenotypic response of the symbiont to changes in the light environment (Falkowski and LaRoche, 1991) and occurs as a result of the symbiont being able to alter the components of its photosynthetic apparatus (Falkowski and LaRoche, 1991; MacIntyre et al., 2002). Thus this finding has implications for bleaching as it suggests that different host-symbiont combinations will have varying photoacclimatory abilities. However, it remains to be seen whether the differential tolerance demonstrated by different host-symbiont associations, translates into differential survival and adaptation to climate change (Venn et al., 2008; Weis, 2008). 
Chapter 1

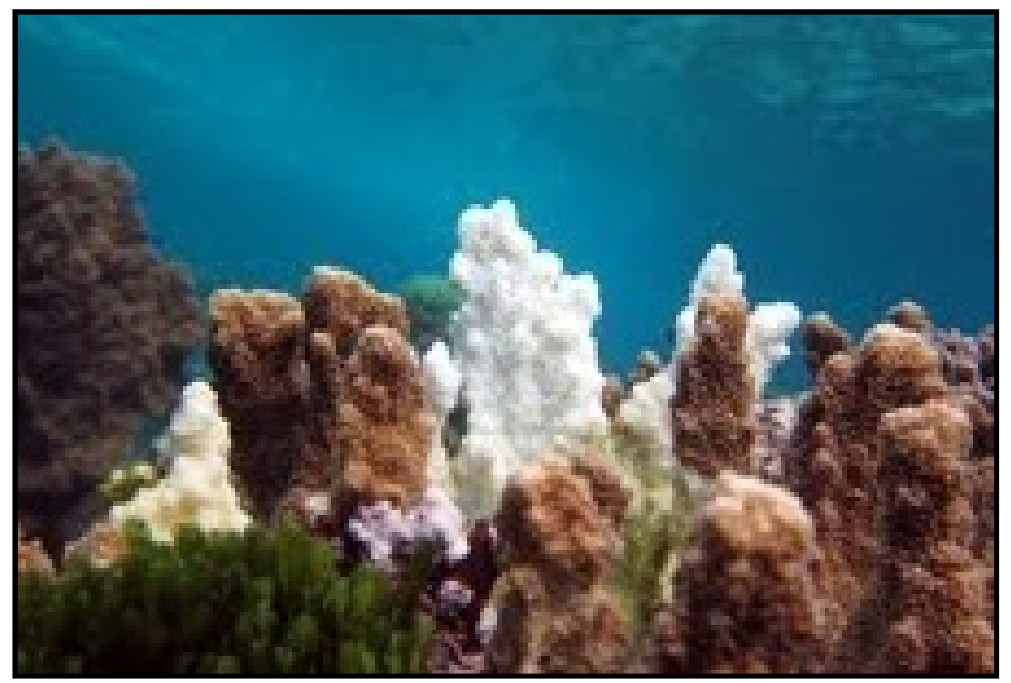

Figure 1.4. Photograph of a partially bleached Acropora palifera colony (obtained from Stefanie Pontasch).

The "adaptive bleaching hypothesis" proposed by Buddemeier and Fautin (1993) is based on the premise that bleaching is a deliberate strategy that allows corals to switch their symbionts as an adaptation to changing environmental conditions. It has generated much controversy in the field (Baker, 2001; Goulet, 2006; HoeghGuldberg et al., 2002) as support for this hypothesis in the wild is still lacking. Although some corals are capable of acquiring symbionts from the water column, this acquisition has been found to be temporary as these infections are not maintained as stable symbioses (Coffroth et al., 2010). And, despite evidence for post-bleaching shifts in the dominant member of a mixed symbiont population (symbiont "shuffling"; Baker, 2001; Baker et al., 2004; Berkelmans and van Oppen, 2006; Mieog et al., 2007; Jones et al., 2008), it is still unknown if the specific hostsymbiont combinations found in nature can change with time at a rate fast enough to match the pace of the changing conditions associated with climate change (Weis, 2008). Figure 1.5 illustrates the proposed symbiont "switching" and "shuffling" mechanisms 
Chapter 1

\subsection{The model system approach}

The fields of molecular, cell and developmental biology have undergone great progress as a result of researchers focusing on a small number of intensively studied model organisms: the bacterium Escherichia coli and its viruses, the yeasts Saccharomyces cervisiae and Schizosaccharomyces pombe, the nematode worm Caenorhabditis elegans, the fruit fly Drosophila melanogaster, the small mustard plant Arabidopsis thaliana, the mouse Mus musculus and a few lines of cultured mammalian cells (reviewed by Davis, 2004; Weis et al., 2008). This model system approach creates rapid progress in a given field by allowing for fast development of new methods and effective collaboration between laboratories (Weis et al., 2008). Recently, research on cnidarian-dinoflagellate symbioses has moved toward the use of a model system. The small tropical anemone Aiptasia sp. that is, like corals, symbiotic with dinoflagellates of the genus Symbiodinium, was selected by investigators as the model system on which to focus (Weis et al., 2008). 


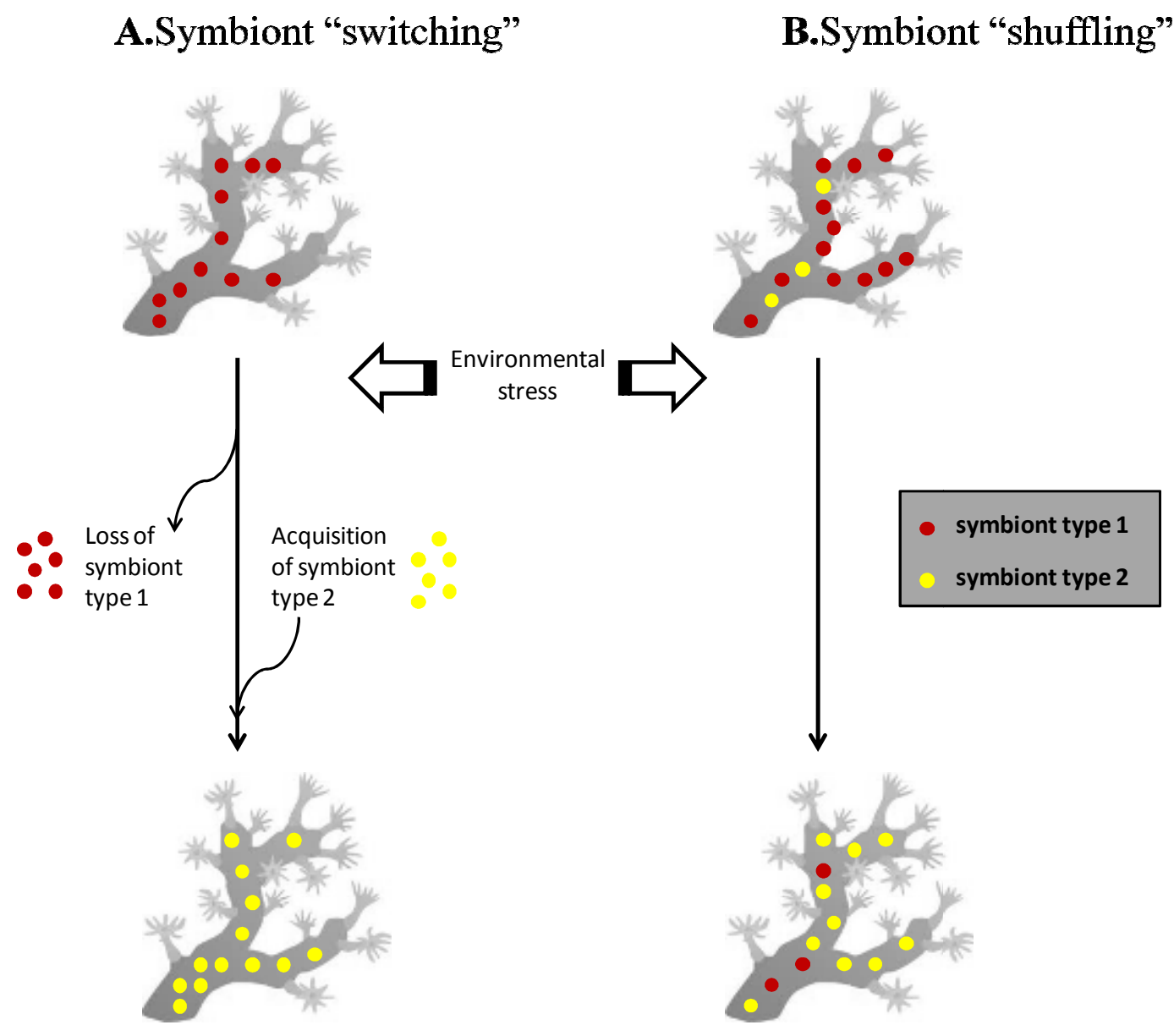

Figure 1.5. Schematic diagram illustrating symbiont (A) "switching" and (B) "shuffling", the two processes that can account for changes in the population of Symbiodinium in a cnidarian host (adapted from Stat et al., 2006).

The two well-studied Aiptasia species are A. pallida (found in the Atlantic) and A. pulchella (found in the Pacific). Individuals from both species undergo sexual and asexual reproduction, and have male and female forms. Sexual reproduction involves the release of gametes into the water column followed by fertilisation and the development of planula larvae. Asexual reproduction, on the other hand, occurs by basal laceration that necessitates the budding of small pieces of tissue from the anemone base (Belda-Baillie et al., 2002).

Aiptasia sp. is a common tropical aquarium pest due to its robust nature and ability to produce large clonal populations. These qualities also contribute to its appropriateness as a model organism. The main advantages of using Aiptasia sp. as a model are that, unlike corals, it can be quickly and easily grown in large numbers in 
Chapter 1

any laboratory making it available for experimentation (Hunter, 1984; Veron, 2000). Because they lack a skeleton, whole anemones can be homogenized or extracted, making Aiptasia sp. accessible for biochemical and molecular biological procedures (Kuo et al., 2004); its cells are also accessible to microscopy (Perez and Weis, 2006). There have also been some promising results from studies with Aiptasia sp. for RNA interference (RNAi) work, which involves suppressing specific genes of interest in order to identify the components necessary for a particular cellular pathway (Perez and Weis, 2006; Dunn et al., 2007). Most importantly, Aiptasia sp. can be fully cleared of its symbionts by temperature shock and dark-treatment (Belda-Baillie et al., 2002; Perez and Weis, 2006), maintained in the symbiont-free state for months or years (Kinzie et al., 2001), and re-infected at will with a variety of Symbiodinium types (Schoenberg and Trench, 1980b; Belda-Baillie et al., 2002) thus making it useful for studies aimed at investigating the cnidarian-dinoflagellate symbiosis (see Figure 1.6).

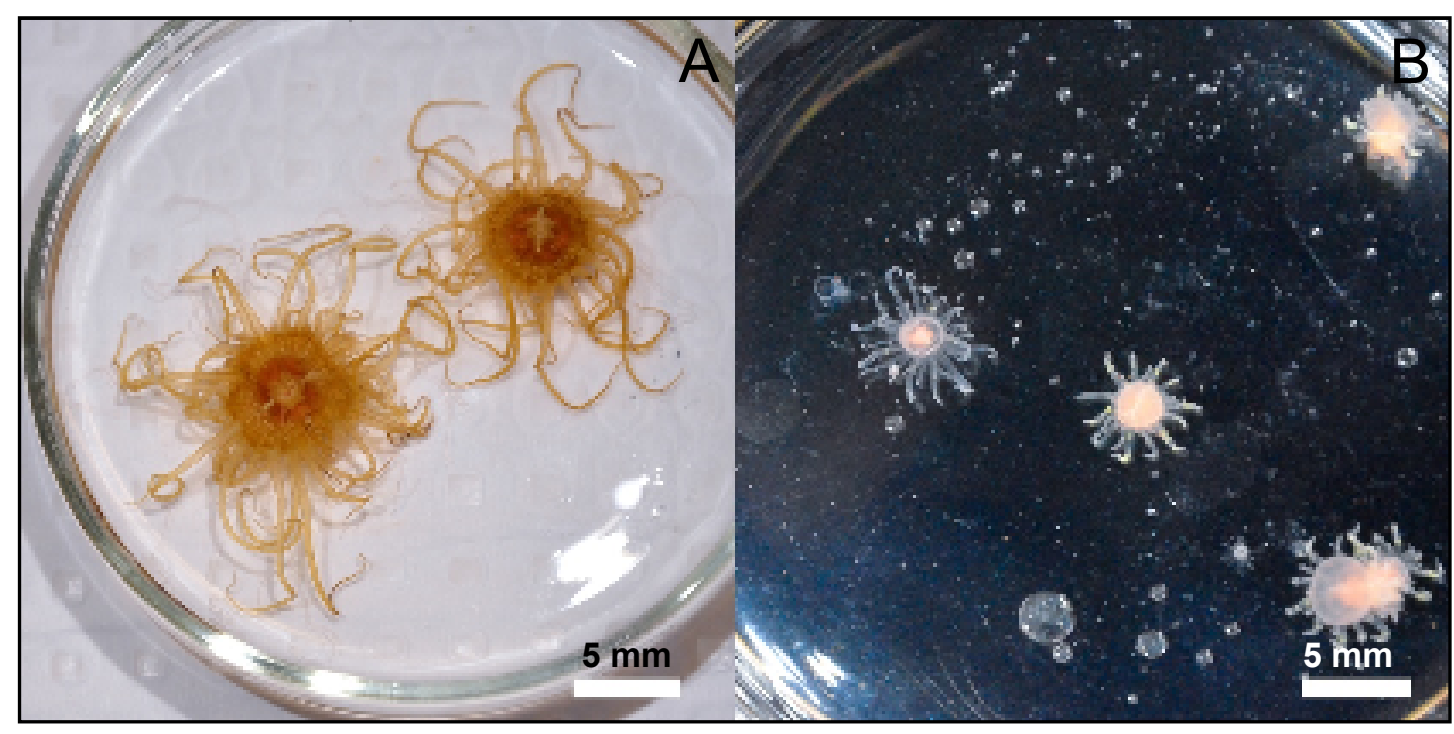

Figure 1.6. Photographs of the model anemone, A. pulchella, in the symbiotic (A) and aposymbiotic (B) state (photographs taken by Tom Hawkins). 
Chapter 1

\subsection{Wider implications and aims}

In order to gain a deeper understanding of how corals can adapt to changing environmental conditions, the effect that symbiont type has on the persistence and physiology of an association needs to be ascertained. This is particularly important when considering the impact of symbiont type on the recovery of an association from an environmental perturbation such as bleaching. Recovery of a symbiosis from such an event will depend on a number of factors: (1) the host's ability to either form a stable association with a novel symbiont type, or to allow a resident symbiont type that has previously been maintained at a low population level to proliferate; (2) the ability of that symbiont to provide enough photosynthetically fixed carbon to meet the host's respiratory demands; (3) the symbiont's ability to provide the required quality of translocate to the host; (4) the host's ability to control the symbiont population growth (and perhaps vice versa); and (5) the ability for both parties to communicate efficiently such that the above four points can be realised. The aim of this research was to determine how different symbiont types affect the nutritional potential and intracellular physiology of the symbiosis when in association with the sea anemone Aiptasia pulchella in order to establish what impact symbiont functional diversity has on the association.

Aim: To test whether Symbiodinium type influences the nutritional potential and intracellular physiology of the cnidarian host.

Objectives:

1. To determine whether different symbiont types are equally as adept at supporting the energetic demands of the same host. Chapter 2 tested the relationship between symbiont type, photosynthetic performance and autotrophic potential in a range of 'normal' and novel cnidariandinoflagellate symbioses.

2. To determine if $\mathrm{pHi}$ is a reflection of symbiont type and whether the optimal $\mathrm{pH}$ for photosynthesis coincides with the host cell $\mathrm{pHi}$. These questions were 
Chapter 1

investigated in Chapter 3 by measuring the host cell $\mathrm{pHi}$ of associations with different symbiont types and assessing whether the optimal $\mathrm{pH}$ of different symbiont types falls within the range of host $\mathrm{pHi}$ values measured.

3. To test whether Symbiodinium type influences the glycerol and glucose pools of the host. Chapter 4 approached this objective by comparing the amounts of free glycerol and glucose (metabolites) in the host tissue of associations with different symbiont types, both prior to, and at different time points after photosynthesis began. 


\section{Chapter 2:}

\section{The influence of symbiont diversity on the photophysiological performance of novel cnidarian-dinoflagellate symbioses}

\subsection{Introduction}

Cnidarian-dinoflagellate symbioses are widespread in the marine environment and are of great ecological importance (Furla et al., 2005). The symbiosis between anthozoan cnidarians (e.g. corals, sea anemones) and dinoflagellates (commonly referred to as zooxanthellae) of the genus Symbiodinium is responsible for the formation of coral reefs which, in turn, maintain a rich biodiversity in shallow tropical waters and thus are vital for sustaining the coastal communities in these regions (Peterson and Lubchenco, 1997; Furla et al., 2005).

The presence of dinoflagellate symbionts contributes substantially to the productivity, survival and success of their cnidarian hosts (Muscatine and Porter, 1977). A mutualistic relationship exists between the host and symbiont, in which the symbiont receives inorganic nutrients from the host and in return provides the host with translocated photosynthetic products (Muscatine, 1990). In most cnidarians, the symbiotic dinoflagellates are intracellular within the endodermis (Schoenberg and Trench, 1980a) and a perisymbiont membrane of animal origin separates them from the animal cytoplasm (Wakefield and Kempf, 2001). The genus Symbiodinium, to which these dinoflagellates belong, is characterised by a high degree of genetic diversity, with nine clades (A-I) and numerous sub-clades currently identified (Coffroth and Santos 2005; Stat et al., 2011).

The carbon fixed by the symbiotic dinoflagellates (probably by the $\mathrm{C} 3$ pathway) is used to support their own respiration and growth (Muscatine et al., 1981; Streamer et al., 1993). However, a portion of this carbon is translocated to the host (Muscatine et al., 1981; Davies, 1984), primarily in the form of glucose, glycerol and lipid 
Chapter 2

(Muscatine, 1967; Trench, 1974; Kellogg and Patton, 1983; Patton et al., 1983; Muscatine et al., 1984; Whitehead and Douglas, 2003; Peng et al., 2011). This translocated carbon is used for host respiration, growth and reproduction, or is released from the association (Muscatine et al., 1981; Edmunds and Davies, 1986; Rinkevich, 1989; McCloskey et al., 1994). It has been shown that up to $99 \%$ of carbon fixed in photosynthesis is translocated to the host (Steen and Muscatine, 1984; McCloskey et al., 1994; Day, 1994; Davy et al., 1996; see review by Muller-Parker and Davy, 2001) and that well in excess of $100 \%$ of the host's daily respiratory carbon demands can be met by autotrophy under well-lit conditions (Muscatine et al., 1981, 1983; Davies, 1991; Davy et al., 1996; Verde and McCloskey, 2002, 2007).

In recent years there has been an increase in the frequency and severity of episodes of coral bleaching (the whitening of corals due to the expulsion of their zooxanthellae and/or the loss of photosynthetic pigments) resulting in degradation and mortality of coral reefs on a global scale (Baker, 2003; Lesser and Farrell, 2004; Obura, 2005). However, our understanding of bleaching, and the potential for corals to withstand or recover from this phenomenon, has been hampered by our limited knowledge of the underlying mechanisms by which the cnidarian-dinoflagellate symbiosis is initiated and maintained (Weis et al., 2008; Weis and Allemand, 2009; Davy et al., 2012). For example, what is the potential for novel host-symbiont combinations to form and persist, and do they function optimally? Buddemeier and Fautin (1993) considered this issue and proposed that, after a bleaching event, corals have the capacity to acquire novel symbionts that are better suited to the new environmental regime than were the previous symbionts (i.e. symbiont 'switching'). Alternatively, lower density symbiont communities already inside the host may, in response to environmental change, increase in numbers and become the dominant component of the symbiont population (i.e. symbiont 'shuffling') (Baker, 2003; Fautin and Buddemeier, 2004). There is experimental evidence for such 'shuffling'in the field (Berkelmans and van Oppen, 2006; Jones et al., 2008), though symbiont 'switching' has so far only been reported conclusively in a laboratory situation (e.g. Schoenberg and Trench, 1980b; Davy et al., 1997; Lewis and Coffroth, 2004). In either case though, the potential for the symbiosis to survive and proliferate will be greatly influenced by the physiological performance of the symbiont type. Past 
studies have provided evidence for this. For example, previous work on induced symbiosis of giant clams with zooxanthellae from various sources (different host species or fast-growing hosts) demonstrated differences in growth and survival rates in the laboratory (Fitt and Trench, 1981; Fitt, 1985), in outdoor culture tanks (Molea and Munro, 1994) and in the field (Belda-Baillie et al., 1999). Also, Stat et al., (2008) demonstrated that differences exist between Symbiodinium types belonging to clade $\mathrm{A}$ and clade $\mathrm{C}$ with respect to the amount of carbon that is fixed and released. However, to date the key question of whether different symbiont types are equally as adept at supporting the energetic demands of the same host species has not been quantified. In order to be able to predict whether novel host-symbiont combinations might arise and succeed in the future, this needs to be investigated.

The relationship between symbiont type, photosynthetic performance and autotrophic potential in a range of 'normal' and novel cnidarian-dinoflagellate symbioses was tested. The sea anemone Aiptasia pulchella was used as the experimental host; this anemone is a widely employed model laboratory system for coral research (Weis et al., 2008). Ultimately, the aim of the study was to gain insight into the influence of symbiont diversity on the nutritional physiology of the symbiosis and on the potential for that symbiosis to survive autotrophically.

\subsection{Materials and methods}

The sea anemone Aiptasia pulchella was used as the experimental host and infected with different Symbiodinium types (freshly-isolated and cultured Symbiodinium B1, heterologous Symbiodinium B1 as well as Symbiodinium A1.4, E2 and F5.1). After 12 weeks the maximum photosynthetic and dark respiratory $\mathrm{O}_{2}$ fluxes were measured in order to calculate a daily carbon budget for the different symbioses tested.

\subsubsection{Experimental organisms}

The sea anemone $A$. pulchella (originally from the Indo-Pacific region) was cultured at $25{ }^{\circ} \mathrm{C}$ and an irradiance of $100 \mu \mathrm{mol}$ photons $\mathrm{m}^{-2} \mathrm{~s}^{-1}(12 \mathrm{~h}$ light:12 h dark) for a 
period of $>1$ year prior to use. The anemones were fed twice weekly with freshlyhatched Artemia sp. over this period. To render the anemones aposymbiotic (i.e. symbiont-free), 120 individuals (oral disc diameter $\sim 10 \mathrm{~mm}$ ) were placed in six 1-L light-proof containers (20 individuals in each) filled with $0.44 \mu \mathrm{m}$ filtered seawater (FSW) and cold shocked at $4{ }^{\circ} \mathrm{C}$ for 24 hours. The cold-shock process was repeated at weekly intervals for five weeks and the feeding regime was retained between cold shocks, when care was taken to keep the anemones in low light $\left(<1 \mu \mathrm{mol}\right.$ photons $\mathrm{m}^{-}$ $\left.2 \mathrm{~s}^{-1}\right)$. The anemones were then maintained in the dark at $25^{\circ} \mathrm{C}$ for approximately six months until the start of the experiment. The aposymbiotic state of the anemones was checked by exposure to light ( $12 \mathrm{~h}$ light:12 h dark) over a four-week period, with any change from white to brown colouration being indicative of a residual symbiont population. Fluorescence microscopy (Olympus Provis AX70 microscope using autofluorescence at $100 \times$ magnification) was used to further screen for the presence of symbionts in a sub-sample of 3 anemones per container (whole anemones were screened). Only anemones that showed no signs of symbiont infection were used for subsequent experiments.

Symbiotic dinoflagellates were freshly isolated from A. pulchella for further infection experiments. Three anemones were homogenised in $1 \mathrm{~mL}$ of FSW in a hand-held, glass tissue grinder for $5 \mathrm{~min}$ and the homogenate then centrifuged at $\times 400 \mathrm{~g}$ for $5 \mathrm{~min}$ to separate the animal and algal fractions. The supernatant was collected and the algal pellet was re-suspended in $100 \mu \mathrm{L}$ of FSW to achieve a density of approximately $10^{6}$ cells $\mathrm{mL}^{-1}$.

Cultures of a range of heterologous Symbiodinium types (i.e. not originally isolated from the $A$. pulchella laboratory culture), as well as a culture of the homologous type that was isolated from the laboratory anemone culture, were grown at $25{ }^{\circ} \mathrm{C}$ and an irradiance of $100 \mu \mathrm{mol}$ photons $\mathrm{m}^{-2} \mathrm{~s}^{-1}$ on a light regime of $12 \mathrm{~h}$ light: $12 \mathrm{~h}$ dark. The heterologous Symbiodinium types (refer to Table 2.1 for details) were selected such that a range of clades was represented. Furthermore, a heterologous B1 type was used to provide a comparison with the homologous B1 zooxanthellae. The zooxanthellae were sub-cultured from long-term laboratory stocks and grown in silica-free F/2 medium (Sigma-Aldrich, Auckland, New Zealand) for six weeks prior 
Chapter 2

to use in the infection study. All Symbiodinium types will be referenced by their cladal designation.

Table 2.1. Sub-clade, original host species and geographic origin of Symbiodinium cultures. Sub-clade genotyping was based on the ITS2 gene.

\begin{tabular}{|l|l|l|l|}
\hline Culture I.D. & Host & Geographical location & Sub-clade \\
\hline FlCass & Cassiopea xamachana & Long Key, Florida & A1.4 \\
\hline $\begin{array}{l}\text { FlAp2 } \\
\text { (heterologous) }\end{array}$ & Aiptasia pallida & Long Key, Florida & B1 \\
\hline Homologous & Aiptasia pulchella & $\begin{array}{l}\text { Pacific } \\
\text { (specific location unknown) }\end{array}$ & B1 \\
\hline CCMP421 & $\begin{array}{l}\text { Free-living (host, if } \\
\text { any, unknown) }\end{array}$ & Wellington Harbour, NZ. & E2 \\
\hline Sin & Sinularia sp. & Guam & F5.1 \\
\hline
\end{tabular}

Symbiodinium types $(\mathrm{n}=3$ samples per culture or isolate) were genetically identified prior to infection (see Appendix 3 for the ITS2 sequences). DNA was extracted from zooxanthellae as described by Logan et al. (2010). Polymerase chain reaction (PCR) was performed using the following Symbiodinium-specific ITS2 primers: forward primer (ITSD), 5'-GTG AAT TGC AGA ACT CCG TG-3'; reverse primer (ITS2rev2), 5'-CCT CCG CTT ACT TAT ATG CTT-3'. Reactions were performed in a total volume of $30 \mu \mathrm{L}$ using the $\mathrm{MyTaq}^{\mathrm{TM}} \mathrm{Mix}$ with an amplification profile consisting of $1 \min 95{ }^{\circ} \mathrm{C}, 35$ cycles of $1 \min 95{ }^{\circ} \mathrm{C}, 15$ s $55{ }^{\circ} \mathrm{C}, 10 \mathrm{~s} 72{ }^{\circ} \mathrm{C}$, and a final hold temperature of $4{ }^{\circ} \mathrm{C}$. Single strand conformation polymorphism (SSCP) was performed on all PCR products. PCR products were sequenced by Macrogen Inc. (Seoul, Korea). Sequences were aligned using the ClustalW plugin in Geneious Pro 4.8.5 and then edited. A blast search was then performed in the National Centre for Biotechnology Information (NCBI) database in order to identify the sequences (see Table A3.1). 


\subsubsection{Infection of aposymbiotic $A$. pulchella with different Symbiodinium types}

Aposymbiotic specimens of $A$. pulchella $(\mathrm{n}=60$ per treatment) were infected with cultured or freshly-isolated Symbiodinium cells by pipetting a concentrated drop of dinoflagellate suspension (approximately 1 million cells $/ \mathrm{mL}$ ) onto the oral disc of each anemone, with a sharp-ended glass pipette. A drop of one-day old Artemia sp. culture was then pipetted onto the oral disc to induce a feeding response and encourage the uptake of the Symbiodinium cells, as described by Davy et al. (1997). Anemones were kept in FSW in $20 \mathrm{~mL}$ glass containers that received a water change 8 hours after infection. Five anemones, all infected with the same symbiont type, were allocated to each container (i.e. 12 containers per treatment). The physiological performance of the different symbioses was then assessed over a 12-week period as described below. The feeding regime during the 12 -week period was maintained as per pre-infection as was the temeprature and light regime. After 12 weeks, zooxanthellae were isolated from three anemones per treatment and their genetic identity confirmed, using the isolation and molecular techniques described above. All treatments were found to harbour the same Symbiodinium type as the one introduced at the start of the experiment (see Appendix 3 for the ITS2 sequences).

\subsubsection{Symbiont growth rate, density, size and biomass}

At Weeks 1, 2, 4, 8 and 12, the growth rate of the zooxanthellae was measured. Individual anemones, each sampled from a different storage container at each time point, ( $\mathrm{n}=4$ per treatment per time point) were homogenised in $1 \mathrm{~mL}$ of FSW in a hand-held, glass tissue grinder for $5 \mathrm{~min}$ and the homogenate then centrifuged at $\times 400 \mathrm{~g}$ for $5 \mathrm{~min}$ to separate the animal and algal fractions. The algal fraction was used to calculate the mitotic index. The percentage of algal cells (out of a total of 300 counted) appearing as doublets (mitotic index; MI) was measured by light microscopy at $400 \times$ magnification every 3 hours over a 24 -h period and used to infer the algal growth rate $\left(\mathrm{d}^{-1}\right)$ (Muscatine et al., 1983).

At these same time-points, the algal fraction of the anemones used for the $\mathrm{O}_{2}$ flux measurements (see below) was used to determine the algal cell density. A 
haemocytometer and light microscope (100× magnification) were used to estimate the total number of algal cells per $\mathrm{mL}$ of anemone homogenate $(\mathrm{n}=6$ counts per anemone), and the total number of algae was normalised to host protein content, measured as described below. Average algal cell diameter $(\mu \mathrm{m} ; \mathrm{n}=20$ cells per anemone per treatment) was also measured by light microscopy (100× magnification), and the cell volume $\left(\mu \mathrm{m}^{3}\right)$ and carbon content $(\mu \mathrm{g})$ were derived from this value (Strathmann, 1967; Muscatine et al., 1981).

\subsubsection{Photosynthesis and respiration}

Maximum photosynthetic and dark respiratory $\mathrm{O}_{2}$ fluxes were measured for each treatment at Weeks 1, 2, 4, 8 and 12. Individual anemones were placed in a glass chamber of volume $10 \mathrm{~mL}$, itself situated in a glass water bath set at $25{ }^{\circ} \mathrm{C}$ (four anemones, each sampled from a different storage container, were used per treatment at each time point to obtain four replicates). The chamber contained FSW that was stirred by a magnetic spin bar that rotated at the maximum speed at which the anemone did not appear stressed; the spin bar was overlaid by a perforated floor of nylon mesh on which the anemone sat. The chamber was sealed by a glass lid with rubber O-ring, into which an oxygen electrode (FIBOX 3 - fiber-optic oxygen meter, PreSens GmbH, Germany) and temperature probe were inserted. These were connected to the oxygen meter. The chamber was illuminated by a Thorn PAR (photosynthetically active radiation) $38150 \mathrm{~W}$ sealed beam-reflector lamp and irradiance incident on the surface of the chamber was measured by a QSL-100 irradiance meter (Biospherical Instruments Inc.). The anemone was left to settle for an hour in the chamber prior to measurement so that it could attach to the grid and open its tentacles. The respiration rate $\left(\mathrm{mL} \mathrm{O}_{2} \mathrm{~h}^{-1}\right)$ was measured in darkness for about an hour, by which time a constant rate was observed. The oxygen tension was not allowed to fall below 50\% saturation (Davies, 1984). The PAR lamp was then switched on and the rate of net photosynthesis by the intact association was measured at $400 \mu \mathrm{mol}$ photons $\mathrm{m}^{-2} \mathrm{~s}^{-1}$ (light-saturation for photosynthesis), again until constant and for about an hour. A preliminary study was performed to check that there was no photoinihibation at $400 \mu \mathrm{mol}$ photons $\mathrm{m}^{-2} \mathrm{~s}^{-1}$. 
The rate of gross photosynthesis was calculated by adding net photosynthesis to dark respiration, and converted into carbon equivalents by assuming photosynthetic and respiratory quotients of 1.1 and 0.9, respectively (Muscatine et al., 1981, 1983; Muscatine, 1990). Animal tissue and zooxanthellae were isolated by homogenisation and centrifugation as described before. The animal supernatant was collected and the algal pellet re-suspended in $1 \mathrm{~mL}$ FSW. Total animal protein was quantified by measuring absorbance of the supernatant at $280 \mathrm{~nm}$ using a ND -1000 spectrophotometer (Nanodrop, Thermo Fisher Scientific Inc.); FSW was used for blank measurements. A preliminary study was performed to assess DNA contamination of the host tissue isolates. The ratio of absorbance at $260 \mathrm{~nm}$ vs 280 $\mathrm{nm}$ was measured for all samples at weeks 8 and 12. As the DNA content in the samples was found to be minimal (less than $5 \%$ in all cases), absorbance at $280 \mathrm{~nm}$ was used to quantify protein content. The total gross photosynthetic and respiration rates were adjusted for the respiration chamber volume and normalised to animal protein content. The algal fraction was used to determine the algal cell density and volume as described previously.

\subsubsection{Carbon budgets}

The contribution of the zooxanthellae to animal respiration (CZAR) was estimated using the "growth rate method" of Muscatine et al. (1981), however a zooxanthellar C:N ratio of 6.1:1 was assumed in the current study (D'Elia et al., 1983). Algal cell protein was derived from carbon content by assuming a $\mathrm{C}: \mathrm{N}$ ratio of 6.1:1 (D'Elia et al., 1983) and that algal cell protein is nitrogen x 6.25 (Muscatine et al., 1983). The "growth rate method" method assumes that all photosynthetic carbon not used for respiration or growth by the symbionts is translocated to the host and hence available to support its metabolism, and is calculated according to the equation: CZAR = $\left[\left(\mathrm{P}_{\mathrm{zNet}} \times \mathrm{T}\right) / \mathrm{R}_{\mathrm{a}}\right] \times 100$, where $\mathrm{P}_{\mathrm{zNet}}$ is net photosynthetic carbon fixation, $\mathrm{T}$ is the percentage of net photosynthetic production translocated to the host (calculated according to the equation: $\mathrm{T}=\left(\mu_{\mathrm{c}}-\mu \mathrm{x} 100\right) / \mu_{\mathrm{c}}$, where $\mu_{\mathrm{c}}$ is the carbon specific growth rate and $\mu$ is the cell specific growth rate), and $\mathrm{R}_{\mathrm{a}}$ is the carbon consumed in animal respiration. This method also assumes that $\mathrm{R}_{\mathrm{z}}$ (zooxanthellar respiration) and $\mathrm{R}_{\mathrm{a}}$ (animal respiration) are proportional to their relative biomasses. The CZAR and 
its associated parameters were estimated as described in detail elsewhere (Muscatine et al., 1981, 1983; Davy et al., 1996), except that a daily light regime of $12 \mathrm{~h}$ saturating irradiance: $12 \mathrm{~h}$ dark was assumed. The CZAR was calculated for all symbioses tested and at all time points.

\subsubsection{Statistical analyses}

Data were tested for normality using the Kolmogorov-Smirnoff test and for homogeneity of variance using the Bartlett test. Multiple comparisons of the various parameters (i.e. algal density, $\mathrm{R}_{\mathrm{a}}, \mathrm{P}_{\text {gross, }}, \mathrm{P}_{\mathrm{zNet}}, \mathrm{MI}, \mathrm{T}$ and CZAR) between the different symbiotic associations and time points were made using one-way analysis of variance (ANOVA, $\alpha=0.05$ ), followed by Least Squares Difference (LSD) post hoc tests for normal data using PASW (Predictive Analytics SoftWare, Version 18). Non-parametric permutation tests were conducted using the $\mathrm{R}$ statistics package, version 2.13.0 (http://www.r-project.org), for data that did not fit the normal distribution. All statistical comparisons provided in the results are one-way ANOVA with post hoc LSD testing unless otherwise stated. Due to high variability that was apparently masking trends for the other symbiont types, Symbiodinium F5.1 was removed from the analyses of algal density and total $\mathrm{P}_{\text {gross }}$ at Week 12.

\subsection{Results}

\subsubsection{Symbiont density}

Patterns of symbiosis establishment varied between the different Symbiodinium types, though all increased significantly between Weeks 1 and 4 (Figure 2.2 and Table 2.3; one-way ANOVA, $\mathrm{p}<0.05$ for all treatments). Densities of Symbiodinium A1.4 (Figure 2.2 C), E2 (Figure 2.2 D) and F5.1 (Figure 2.2 B), and both the freshlyisolated (Figure $2.2 \mathrm{E}$ ) and cultured homologous (Figure $2.2 \mathrm{~F}$ ) zooxanthellae (Symbiodinium B1), increased by 67-95\% between Weeks 1 and 4 (LSD, p $<0.05$ ), before levelling off between Weeks 4 and 12 (LSD, p >0.05). The heterologous Symbiodinium B1 (Figure 2.2 A) density increased by 91\% between Weeks 1 and 4, 
Chapter 2

stabilised between Weeks 4 and 8 (LSD, p > 0.05) but then declined by 70\% between Weeks 8 and 12 (LSD, $\mathrm{p}<0.05$ ).

At Week 12 and Week 1, significant differences were apparent between the densities of the various Symbiodinium types, though, in the case of Week 12, only once the highly variables values for type F5.1 (Figure $2.2 \mathrm{~B}$ ) had been excluded from the analysis (Table 2.4; one-way ANOVA, $\mathrm{p}<0.005$; see Appendix 1, Table A1.1). At Week 1, Symbiodinium E2 (Figure 2.2 D) displayed a significantly higher density than all the other types (LSD, $\mathrm{p}<0.005$ ). At Week 12, Symbiodinium E2 (Figure 2.2 D) and A1.4 (Figure 2.2 C) displayed significantly higher densities than did the others (LSD, $\mathrm{p}<0.05$ ). These differences led to similar trends in the symbiosis biomass ratio (algal:total protein) ratio, which was again significantly different between various host-symbiont combinations (one-way ANOVA, d.f. $=19, \mathrm{~F}=4.6$, $\mathrm{p}<0.05$ ). Specifically, the biomass contribution of Symbiodinium E2 (Figure 2.1 D) was greater than that of all the B1 types (i.e. heterologous, and freshly-isolated and cultured homologous; Figure $2.2 \mathrm{~A}, \mathrm{E}$ and $\mathrm{F}$ respectively), and the biomass contribution of Symbiodinium A1.4 (Figure $2.2 \mathrm{C}$ ) was greater than that of the cultured homologous and heterologous B1 types (Figure 2.2 E and F; LSD, p $<0.05$ for all comparisons).

\subsubsection{Symbiont mitotic index (MI)}

All Symbiodinium types displayed a daily synchronicity in the timing of cell division (MI; see Appendix 1, Table A1.1). A peak in the MI was noted at 7 am (the start of the light period) for all types except the cultured homologous Symbiodinium, which displayed a peak at $10 \mathrm{am}$, and these patterns were consistent across the 12-week period (see Figure 2.1 for daily Week 1 MI graphs). However, the average daily MI decreased significantly with time for all Symbiodinium types during the 12-week experiment (Table 2.1; one-way ANOVA, $\mathrm{p}<0.05$ for all treatments), with the MI at Week $1(2.3-4.4 \%)$ being significantly greater than at the subsequent time-points $(0.009-1.9 \%)$ in all cases (LSD, $\mathrm{p}<0.05)$. 
Chapter 2

There were differences between the average MI of the different Symbiodinium types at some of the time-points (Table 2.4; one-way ANOVA, $\mathrm{p}<0.05$ ). In particular, the average MI at Week 1 was higher for all of the B1 types (i.e., heterologous, and freshly-isolated and cultured homologous) than for the other types (LSD, p < 0.05), while at Week 12 the cultured homologous B1 had a lower MI than all of the other types (Table 2.2; LSD, p <0.005).
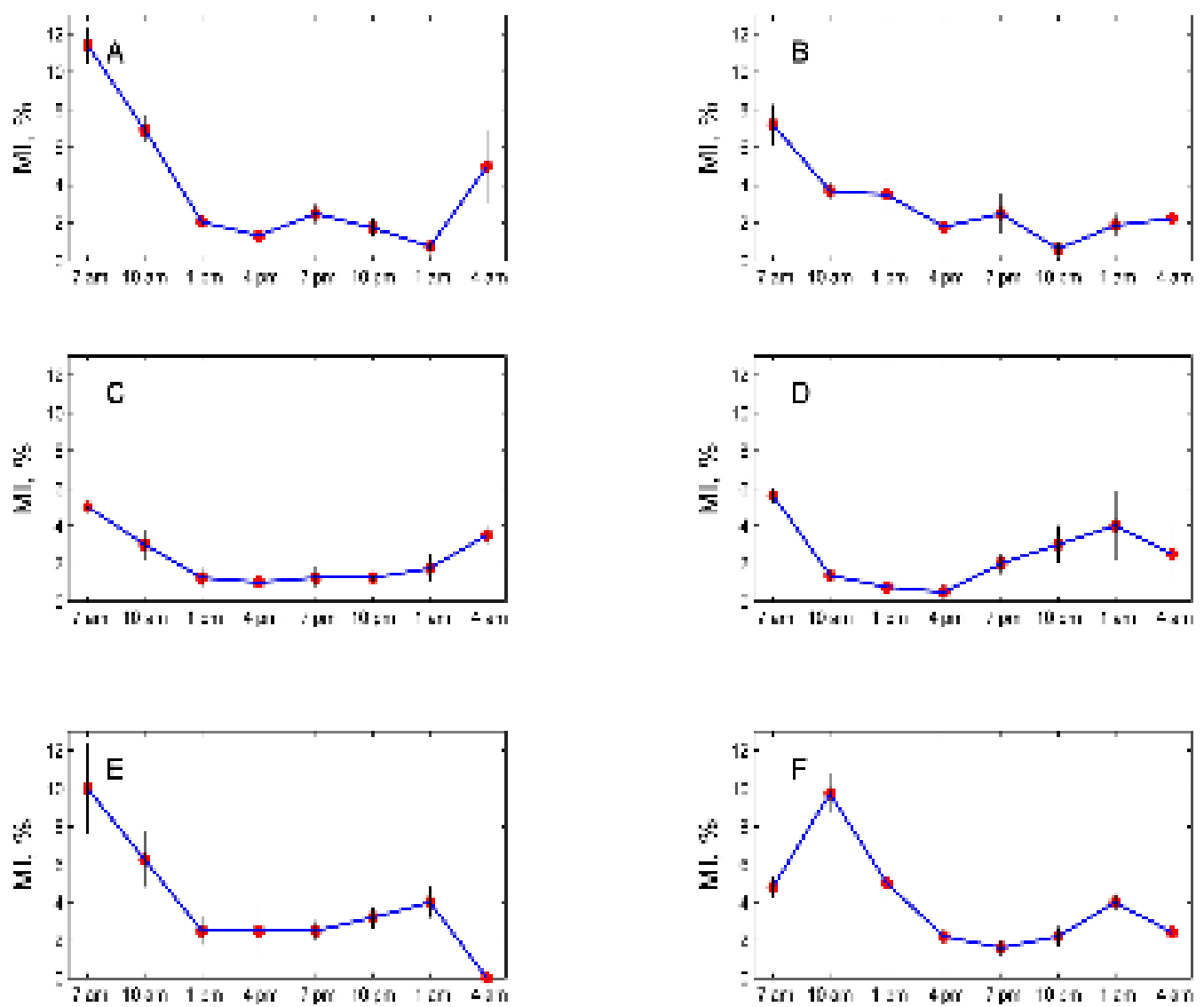

Figure 2.1. The Mitotic Index (MI) in A. pulchella calculated over a 24 hour period at Week 1 by a range of different cultured heterologous Symbiodinium types: (A) heterologous B1, (B) F5.1, (C) A1.4, and (D) E2. A. pulchella was also infected with homologous Symbiodinium type B1 that was either (E) freshly isolated or $(F)$ cultured. Values are means \pm SE; $n=5$ per time point. 
Chapter 2

\subsubsection{Photosynthesis, respiration and $P: R$}

The algal biomass specific rate of gross photosynthesis $\left(\mathrm{P}_{\text {gross }}, \mu \mathrm{g} \mathrm{Cm}^{-3}\right.$ day $\left.^{-1}\right)$ for the symbioses involving Symbiodinium A1.4, B1 (heterologous) and F5.1, as well as both the freshly isolated and cultured homologous Symbiodinium B1, decreased over the 12-week post-infection period (Figure 2.2 C, A, B, E and F respectively, Table 2.3; one-way ANOVA, $\mathrm{p}<0.05$ ). There was a significant decrease in $\mathrm{P}_{\text {gross }}$ between: Week 2 and Weeks 8-12 for Symbiodinium A1.4 (Figure 2.2 C); Week 1 and Weeks 4-12 for the heterologous Symbiodinium B1 (Figure 2.2 A); Week 2 and Weeks 4-12 for Symbiodinium F5.1 (Figure 2.2 B); Week 1 and Weeks 2-8 for the freshly isolated homologous B1 (Figure 2.2 E); and Week 1 and Weeks 2-12 for the cultured homologous B1 (Figure 2.2 F) (LSD, p $<0.05$ for all comparisons). Of note, however, $\mathrm{P}_{\text {gross }}$ in Symbiodinium A1.4 (Figure $2.2 \mathrm{C}$ ) actually increased between Weeks 1 and 2 (LSD, p < 0.05) despite its subsequent decline, while $\mathrm{P}_{\text {gross }}$ in Symbiodinium E2 (Figure 2.2 D) remained unchanged throughout the experimental period (LSD, $\mathrm{p}>0.05$ ). Nevertheless, at Week 12, no significant difference in $\mathrm{P}_{\text {gross }}$ was observed between the Symbiodinium types (Table 2.4; one-way ANOVA, d.f. = $16, \mathrm{~F}=2.7, \mathrm{p}>0.05$ for all comparisons).

The total photosynthetic rate for the symbiosis $\left(\mu \mathrm{g} \mathrm{C} \mathrm{mg}^{-1}\right.$ animal protein day $\left.{ }^{-1}\right)$ did not change over the course of the 12 weeks for any of the symbiont types except for Symbiodinium A1.4 (Figure 2.3, Table 2.3; one-way ANOVA, p $<0.005$ ), which displayed a significant increase between Weeks 1 and 2 (LSD, p $<0.005)$ followed by a significant decrease between Weeks 2 and 8 (LSD, p $<0.05$ ). It appears that the lack of a significant increase in total $\mathrm{P}_{\text {gross }}$ for Symbiodinium B1, as well as Symbiodinium F5.1, may be a result of the effect of the lower initial algal density being countered by a high initial cell specific $\mathrm{P}_{\text {gross }}$ (Figure 2.2). At Week 12, the total photosynthetic rate in the different symbioses was dissimilar (Table 2.2, Table 2.4; one-way ANOVA, d.f. $=4 ; 12, \mathrm{~F}=4.2, \mathrm{p}<0.05$ ), with the symbiosis with Symbiodinium E2 (Figure $2.3 \mathrm{D}$ ) having a significantly higher photosynthetic rate than the other symbioses (LSD, p < 0.05); of note, while not included in this analysis due to its very high variability, the average $\mathrm{P}_{\text {gross }}$ of Symbiodinium F5.1 (Figure 2.3 B) was comparable to that of type E2. 
Chapter 2
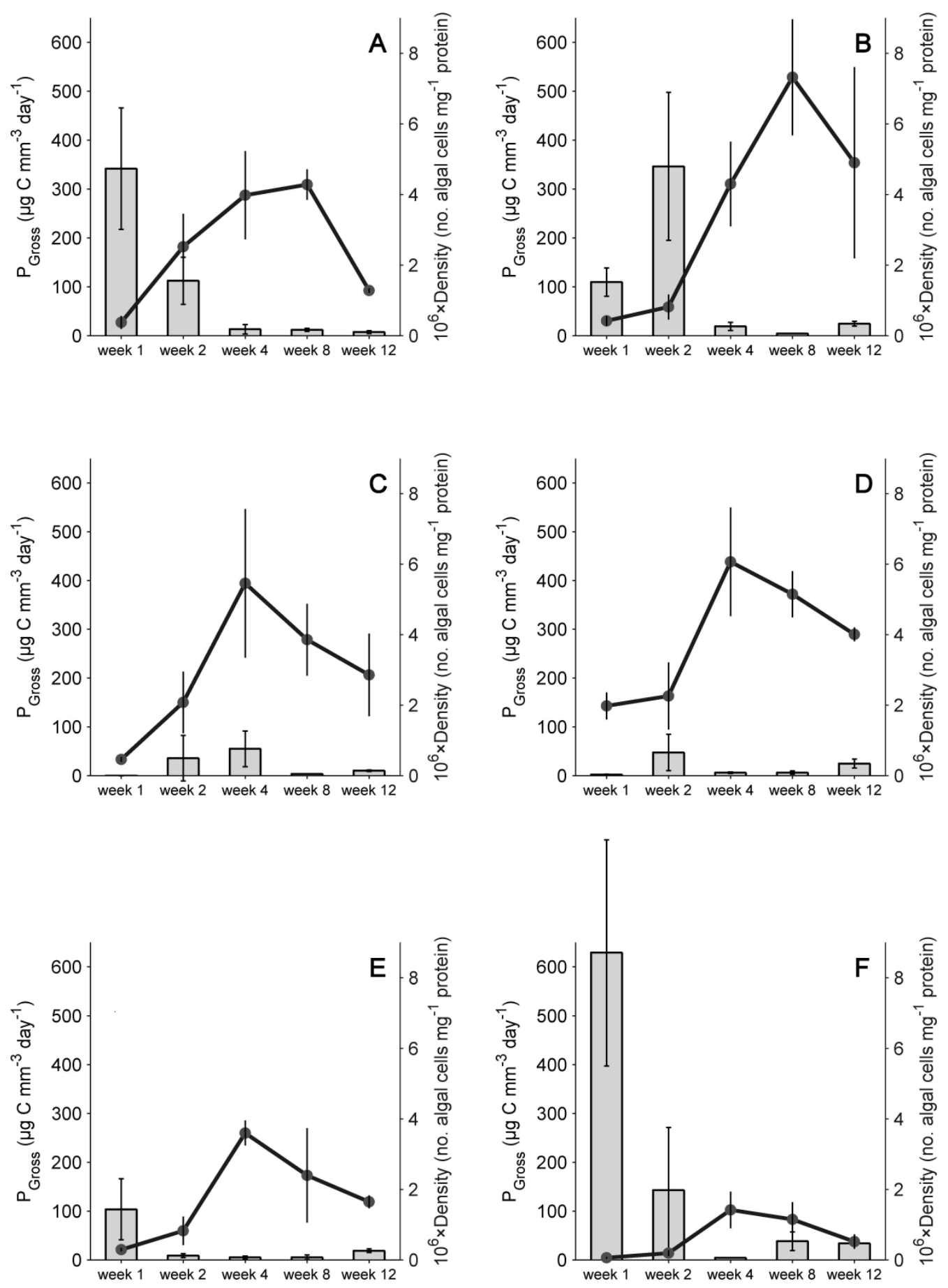

Figure 2.2. Symbiont density (solid line) and cell-specific gross photosynthesis (grey bars) in A. pulchella during infection by a range of different cultured heterologous Symbiodinium types: (A) B1, (B) F5.1, (C) A1.4, and (D) E2. A. pulchella was also infected with homologous Symbiodinium type B1 that was either (E) freshly isolated or (F) cultured. Values are means $\pm S E ; n=4-5$ per time point. 
Chapter 2

Two general trends were observed with regards to symbiosis respiration $\left(\mathrm{R}_{\mathrm{s}}\right)($ Figure 2.3). First, in three cases (Symbiodinium F5.1, A1.4 and the freshly-isolated homologous B1) $\mathrm{R}_{\mathrm{s}}$ changed with time since infection (Table 2.3; one-way ANOVA, $\mathrm{p}<0.05$ ), with an initial increase between Weeks 1 and 2 (A1.4, B1; Figure 2.3 C and A) or 4 (F5.1; Figure 2.3 B), and a subsequent decline to Week 12 (LSD, p < 0.05 and $\mathrm{p}<0.005$ for Symbiodinium A1.4 and B1, and F5.1, respectively). In the second group of remaining Symbiodinium types, $\mathrm{R}_{\mathrm{s}}$ did not change with time (Table 2.3; one-way ANOVA, $\mathrm{p}>0.05$ for all types). At Week 12, significant differences in $\mathrm{R}_{\mathrm{s}}$ were noted between the associations harbouring the different symbiont types (Table 2.4; one-way ANOVA, $\mathrm{p}<0.05$ ), with Symbiodinium E2 and F5.1 generating significantly higher $\mathrm{R}_{\mathrm{s}}$ values than the other types (LSD, $\mathrm{p}<0.05$ ). Animal and zooxanthellar respiration $\left(\mathrm{R}_{\mathrm{a}}, \mathrm{R}_{\mathrm{z}}\right)$ followed similar trends (see Appendix 1, Table A1.1 and Table 2.3 and 2.4), though of note $R_{z}$ varied hugely at Week 12 (from 0.24 to $7.3 \mu \mathrm{g} \mathrm{C} \mathrm{mg}{ }^{-1}$ animal protein day ${ }^{-1}$; one-way ANOVA, $\left.\mathrm{p}<0.005\right)$. This was largely a result of the significantly higher density of Symbiodinium E2 (see above) and the higher rate of $R_{s}$ in this association, which led to a significantly higher $R_{z}$ than for the other symbiont types (LSD, $\mathrm{p}<0.005)$.

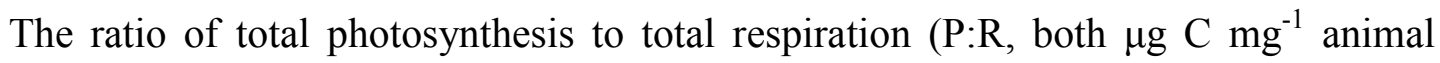
protein day $^{-1}$; Figure 2.3) gives an indication of autotrophic potential. All the associations, except for those involving Symbiodinium E2 and F5.1 (which had much the highest rates of $R_{s}$ ) reached a P:R ratio of approximately 1 or higher by Week 12 , and hence were autotrophic or near-autotrophic with respect to carbon. The association involving the heterologous Symbiodinium B1 attained a value of $>1$ by Week 4, while the freshly-isolated and cultured homologous Symbiodinium B1 reached a $P: R$ ratio of $\sim 1$ by Week 8 . 

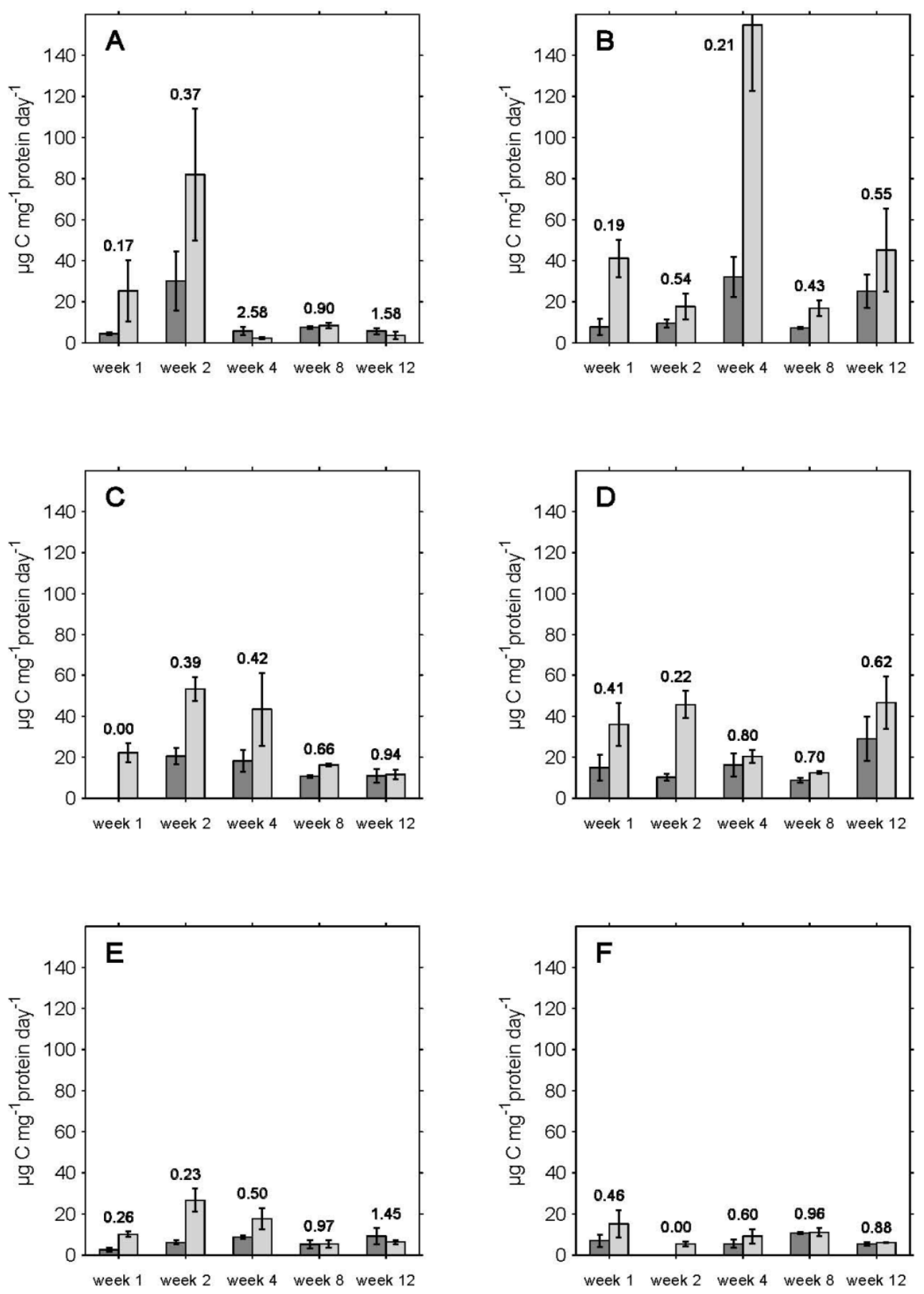

Figure 2.3. Gross photosynthesis (dark grey bars) and total symbiosis respiration (light grey bars) in an A. pulchella - Symbiodinium symbiosis during infection by a range of different cultured heterologous Symbiodinium types: (A) B1 (heterologous), (B) F5.1, (C) A1.4, and (D) E2. A. pulchella was also infected with homologous Symbiodinium type B1 that was either $(\mathrm{E})$ freshly isolated or $(\mathrm{F})$ cultured. The values above the bars are the P:R ratios (i.e. gross photosynthesis/total respiration). Values are means \pm SE; $n=4-5$ per time point. 
Chapter 2

\subsubsection{Translocation}

Symbioses with Symbiodinium E2 and the cultured homologous B1 displayed no significant change in the percentage of carbon available for translocation $(\mathrm{T})$ over the course of reinfection (see Appendix 1, Table A1.1, Table 2.3; one-way ANOVA, p > 0.05). In the other symbioses, however, $\mathrm{T}$ declined significantly with time (see Appendix 1, Table A1.1; one-way ANOVA, $\mathrm{p}<0.05$ for all treatments), in part because of calculated increases in $\mathrm{R}_{\mathrm{z}}$ (see above and Appendix 1, Table A1.1). Symbiodinium A1.4 and the freshly-isolated homologous Symbiodinium B1 showed a decrease of $3 \%$ and $30 \%$, respectively between Weeks 1 and 4 , while the heterologous Symbiodinium B1 and Symbiodinium F5.1 displayed a decrease of 9.3\% and 5\%, respectively, between Weeks 1 and 8 (LSD, p $<0.05$ for all comparisons). At Week 12, the cultured homologous Symbiodinium B1 displayed a significantly higher translocation rate (99.9\%) than did Symbiodinium B1 (heterologous), E2 and A1.4, and the freshly-isolated homologous B1 (96.4 - 98.8\%; Table 2.4; one-way ANOVA with post hoc LSD, d.f $=19, \mathrm{~F}=3.7, \mathrm{p}<0.05$ for all comparisons); there were no other significant differences (Table 2.2).

\subsubsection{CZAR}

All the symbioses displayed a significant increase in the CZAR between Weeks 1 and 12 (ANOVA permutation test, $\mathrm{p}<0.05$ for all treatments; see Appendix 1, Table A1.1). The CZAR values obtained at Week 12 were: $176.8 \%$ for the freshly-isolated homologous Symbiodinium B1; $113.0 \%$ for the heterologous B1; $81.1 \%$ for the cultured homologous B1; and 74.9\%, 72.4\% and 52.3\% for Symbiodinium A1.4, E2 and F5.1, respectively (Table 2.2). Despite this broad range of values, however, they were not significantly different (ANOVA permutation test, $p>0.05$ ), most likely due to the high variability in the data for some of the associations. 
Table 2.2. The CZAR in A. pulchella 12 weeks after infection with different Symbiodinium types. Density zooxanthellar cell density; MI mitotic index; $\mu$ cell-specific growth rate; $P_{\text {gross }}$ gross photosynthetic rate; $R_{S}$ symbiosis respiration per day; $P: R$ ratio of total photosynthesis to total respiration; $R_{a}$ derived animal respiration per day; $R_{z}$ derived zooxanthellar respiration per day; $P_{z N e t}$ net photosynthesis per day by zooxanthellae; $\mu_{c}$ carbon-specific growth rate (fraction of the carbon standing stock added per day); $T$ percentage of the net photosynthetic production translocated to host; CZAR potential contribution by zooxanthellae to animal's daily respiratory carbon requirements. The results represent an idealised day of 12 hours light exposure at saturating irradiance and 12 hours of darkness. Values are means $\pm \mathrm{SE}(\mathrm{n}=4-5$ for all parameters, except algal cell diameter, where $\mathrm{n}=72$ and $M I$, where $\mathrm{n}=32$ ).

\begin{tabular}{|c|c|c|c|c|c|c|}
\hline Symbiodinium type & A1.4 & B1 & E2 & F5.1 & $\begin{array}{l}\text { Homologous } \\
\text { (B1) - freshly- } \\
\text { isolated }\end{array}$ & $\begin{array}{l}\text { Homologous } \\
\text { (B1) - cultured }\end{array}$ \\
\hline $\begin{array}{l}\text { Density }\left(\times 10^{6} \text { cells }\right. \\
\left.\mathrm{mg}^{-1} \text { protein }\right)\end{array}$ & $2.9 \pm 1.2$ & $1.3 \pm 0.1$ & $4.1 \pm 0.2$ & $4.5 \pm 2.9$ & $1.6 \pm 0.2$ & $0.5 \pm 0.2$ \\
\hline $\begin{array}{l}\text { Algal cell diameter } \\
(\mu \mathrm{m})\end{array}$ & $9.59 \pm 0.25$ & $9.20 \pm 0.15$ & $8.59 \pm 0.30$ & $7.72 \pm 0.08$ & $8.67 \pm 0.07$ & $8.29 \pm 0.09$ \\
\hline $\begin{array}{l}\text { Derived algal } \\
\left.\text { carbon (pg C cell }{ }^{-1}\right)\end{array}$ & $73.14 \pm 3.53$ & $64.92 \pm 2.83$ & $54.69 \pm 4.30$ & $41.23 \pm 1.06$ & $55.89 \pm 1.06$ & $49.14 \pm 2.71$ \\
\hline MI (\%) & $1.1 \pm 0.2$ & $1.75 \pm 0.6$ & $1.4 \pm 0.5$ & $1.1 \pm 0.2$ & $1.6 \pm 0.3$ & $\begin{array}{l}6.7 \times 10^{-3} \pm \\
1.3 \times 10^{-3}\end{array}$ \\
\hline$\mu\left(d^{-1}\right)$ & 0.060 & 0.018 & 0.017 & 0.015 & 0.020 & $2 \times 10^{-5}$ \\
\hline $\begin{array}{l}P_{\text {gross }}\left(\mu \mathrm{g} \mathrm{C} \mathrm{mg}^{-1}\right. \\
\text { protein day } \\
-1)\end{array}$ & $10.88 \pm 3.41$ & $5.71 \pm 1.46$ & $29.02 \pm 10.70$ & $25.04 \pm 8.10$ & $9.22 \pm 3.87$ & $5.41 \pm 0.71$ \\
\hline $\begin{array}{l}R_{s}\left(\mu g \mathrm{C} \mathrm{mg}^{-1}\right. \\
\text { protein day } \\
\left.{ }^{-1}\right)\end{array}$ & $11.63 \pm 2.40$ & $3.61 \pm 1.80$ & $46.58 \pm 12.79$ & $45.13 \pm 20.25$ & $6.36 \pm 1.00$ & $6.13 \pm 0.22$ \\
\hline $\mathbf{P}: \mathbf{R}$ & 0.94 & 1.58 & 0.62 & 0.55 & 1.45 & 0.88 \\
\hline $\begin{array}{l}\text { Algal:total protein } \\
\text { ratio } \times 10^{-2}\end{array}$ & $15.93 \pm 5.07$ & $5.45 \pm 0.88$ & $17.24 \pm 1.73$ & $18.7 \pm 5.10$ & $6.56 \pm 0.14$ & $3.53 \pm 0.97$ \\
\hline $\begin{array}{l}\mathrm{R}_{\mathrm{a}}\left(\mu \mathrm{g} \mathrm{C} \mathrm{mg^{-1 }}\right. \\
\left.\text { protein } \text { day }^{-1}\right)\end{array}$ & $9.34 \pm 1.02$ & $3.43 \pm 0.56$ & $39.29 \pm 9.70$ & $44.56 \pm 17.82$ & $5.78 \pm 0.74$ & $5.91 \pm 0.18$ \\
\hline $\begin{array}{l}R_{z}\left(\mu g \mathrm{C} \mathrm{mg}^{-1}\right. \\
\text { protein day } \\
-1\end{array}$ & $2.29 \pm 1.25$ & $0.34 \pm 0.20$ & $7.30 \pm 1.85$ & $0.57 \pm 0.39$ & $0.58 \pm 0.19$ & $0.24 \pm 0.08$ \\
\hline $\begin{array}{l}\text { Algal carbon } \\
\text { standing stock: } \mathrm{C}^{\prime} \\
\left(\mu \mathrm{g} \mathrm{C} \mathrm{mg}^{-1} \text { protein) }\right.\end{array}$ & $21.33 \pm 8.21$ & $6.11 \pm 1.04$ & $22.15 \pm 2.74$ & $7.03 \pm 1.44$ & $7.48 \pm 1.72$ & $3.89 \pm 1.12$ \\
\hline $\begin{array}{l}P_{z \text { zet }}\left(\mu \mathrm{g} \mathrm{mg}^{-1}\right. \\
\text { protein day } \\
-1\end{array}$ & $9.5 \pm 3.5$ & $5.1 \pm 1.2$ & $27.0 \pm 10.2$ & $19.6 \pm 5.6$ & $8.8 \pm 3.7$ & $5.4 \pm 0.7$ \\
\hline$\mu_{c}\left(d^{-1}\right)$ & $0.5 \pm 0.09$ & $0.83 \pm 0.10$ & $1.2 \pm 0.40$ & $1.63 \pm 0.40$ & $1.08 \pm 0.20$ & $1.71 \pm 0.43$ \\
\hline T (\%) & $96.4 \pm 0.5$ & $97.5 \pm 0.6$ & $97.4 \pm 1.4$ & $98.8 \pm 0.5$ & $98.0 \pm 0.5$ & $99.9 \pm 0.001$ \\
\hline CZAR (\%) & $74.9 \pm 12.1$ & $113.0 \pm 10.9$ & $72.4 \pm 26.2$ & $52.3 \pm 8.3$ & $176.8 \pm 26.1$ & $81.1 \pm 7.1$ \\
\hline
\end{tabular}


Chapter 2

Table 2.3. One-way ANOVA results for parameters; Density, MI (Mitotic Index), Volumespecific $\mathrm{P}_{\text {Gross }}$, Total $\mathrm{P}_{\text {Gross, }}, \mathrm{R}_{\mathrm{s}}$ (symbiosis respiration), $\mathrm{R}_{\mathrm{z}}$ (zooxanthellar respiration) and $\mathrm{T}$ (translocation) indicating d.f., F values and $\mathrm{p}$ values for each Symbiodinium type.

\begin{tabular}{|c|c|c|c|c|}
\hline Parameter & Symbiont type & d.f. & $\mathbf{F}$ & $\mathbf{p}$ \\
\hline \multirow[t]{6}{*}{ Density } & Heterologous B1 & $4 ; 15$ & 5.7 & $p=0.005$ \\
\hline & FI -Homologous B1 & $4 ; 11$ & 3.4 & $p=0.048$ \\
\hline & C - Homologous B1 & $4 ; 15$ & 7.1 & $p=0.002$ \\
\hline & A1.4 & $4 ; 14$ & 3.4 & $p=0.049$ \\
\hline & E2 & $4 ; 14$ & 3.3 & $p=0.048$ \\
\hline & F5.1 & $4 ; 13$ & 5.6 & $p=0.008$ \\
\hline \multirow[t]{6}{*}{ MI } & Heterologous B1 & $4 ; 155$ & 4.7 & $\mathrm{p}=0.0001$ \\
\hline & FI -Homologous B1 & $4 ; 152$ & 8.5 & $p=0.0001$ \\
\hline & C - Homologous B1 & $4 ; 195$ & 64.1 & $p=0.0001$ \\
\hline & A1.4 & $4 ; 151$ & 2.7 & $\mathrm{P}=0.030$ \\
\hline & E2 & $4 ; 155$ & 2.7 & $p=0.031$ \\
\hline & F5.1 & $4 ; 155$ & 11.6 & $p=0.0001$ \\
\hline \multirow[t]{6}{*}{ Volume specific $\mathrm{P}_{\text {Gross }}$} & Heterologous B1 & $4 ; 14$ & 4.0 & $p=0.023$ \\
\hline & FI -Homologous B1 & $4 ; 10$ & 3.0 & $p=0.050$ \\
\hline & C - Homologous B1 & $4 ; 10$ & 5.9 & $p=0.010$ \\
\hline & $\mathrm{A} 1.4$ & $4 ; 12$ & 3.1 & $p=0.048$ \\
\hline & E2 & $4 ; 10$ & 0.6 & $p=0.686$ \\
\hline & F5.1 & $4 ; 10$ & 3.0 & $p=0.050$ \\
\hline \multirow[t]{6}{*}{ Total $\mathrm{P}_{\text {Gross }}$} & Heterologous B1 & $4 ; 13$ & 2.0 & $p=0.142$ \\
\hline & FI -Homologous B1 & $4 ; 10$ & 1.6 & $p=0.248$ \\
\hline & C - Homologous B1 & $4 ; 15$ & 2.2 & $p=0.117$ \\
\hline & A1.4 & $4 ; 11$ & 10.6 & $p=0.001$ \\
\hline & E2 & $4 ; 11$ & 2.7 & $p=0.083$ \\
\hline & F5.1 & $4 ; 10$ & 1.7 & $\mathrm{p}=0.225$ \\
\hline \multirow[t]{6}{*}{$\mathrm{R}_{\mathrm{s}}$} & Heterologous B1 & $4 ; 10$ & 2.9 & $p=0.078$ \\
\hline & FI -Homologous B1 & $4 ; 9$ & 5.3 & $p=0.018$ \\
\hline & C - Homologous B1 & $4 ; 12$ & 1.8 & $p=0.194$ \\
\hline & A1.4 & $4 ; 11$ & 4.2 & $p=0.027$ \\
\hline & E2 & $4 ; 11$ & 3.0 & $p=0.064$ \\
\hline & F5.1 & $4 ; 9$ & 13.1 & $p=0.001$ \\
\hline \multirow[t]{6}{*}{$\mathrm{R}_{\mathrm{z}}$} & Heterologous B1 & $4 ; 10$ & 4.5 & $p=0.024$ \\
\hline & FI -Homologous B1 & $4 ; 12$ & 8.1 & $p=0.072$ \\
\hline & C - Homologous B1 & $4 ; 13$ & 15.9 & $\mathrm{p}=0.097$ \\
\hline & A1.4 & $4 ; 13$ & 2.4 & $p=0.0001$ \\
\hline & E2 & $4 ; 10$ & 3.0 & $p=0.102$ \\
\hline & F5.1 & $4 ; 12$ & 2.5 & $p=0.002$ \\
\hline \multirow[t]{6}{*}{$\mathrm{T}$} & Heterologous B1 & $4 ; 15$ & 4.6 & $p=0.012$ \\
\hline & FI -Homologous B1 & $4 ; 13$ & 4.0 & $p=0.030$ \\
\hline & C - Homologous B1 & $4 ; 10$ & 1.1 & $\mathrm{p}=0.390$ \\
\hline & A1.4 & $4 ; 14$ & 3.8 & $p=0.042$ \\
\hline & E2 & $4 ; 10$ & 0.7 & $p=0.629$ \\
\hline & F5.1 & $4 ; 15$ & 4.6 & $\mathrm{p}=0.018$ \\
\hline
\end{tabular}


Chapter 2

Table 2.4. One-way ANOVA results for parameters; Density, MI (Mitotic Index), Volumespecific $\mathrm{P}_{\text {Gross }}$, Total $\mathrm{P}_{\text {Gross, }}, \mathrm{R}_{\mathrm{S}}$ (symbiosis respiration), $\mathrm{R}_{\mathrm{z}}$ (zooxanthellar respiration) and $\mathrm{T}$ (translocation) indicating d.f., $F$ values and $\mathrm{p}$ values for each time point.

\begin{tabular}{|l|l|l|l|l|}
\hline Parameter & Week & d.f. & $\mathbf{F}$ & $\mathbf{P}$ \\
\hline Density & 1 & $5 ; 17$ & 12.9 & $\mathrm{p}=0.0001$ \\
\hline & 2 & $5 ; 18$ & 1.2 & $\mathrm{p}=0.333$ \\
\hline & 4 & $5 ; 17$ & 2.0 & $\mathrm{p}=0.129$ \\
\hline & 8 & $5 ; 16$ & 1.9 & $\mathrm{p}=0.143$ \\
\hline & 12 & $4 ; 12$ & 6.9 & $\mathrm{p}=0.004$ \\
\hline MI & 1 & $5 ; 203$ & 8.5 & $\mathrm{p}=0.024$ \\
\hline & 2 & $5 ; 194$ & 0.2 & $\mathrm{p}=0.953$ \\
\hline & 4 & $5 ; 198$ & 8.1 & $\mathrm{p}=0.0001$ \\
\hline & 8 & $5 ; 190$ & 8.5 & $\mathrm{p}=0.0001$ \\
\hline & 12 & $5 ; 178$ & 12.2 & $\mathrm{p}=0.0001$ \\
\hline Volume specific $\mathrm{P}_{\text {Gross }}$ & 1 & $5 ; 13$ & 4.2 & $\mathrm{p}=0.017$ \\
\hline & 2 & $5 ; 12$ & 2.5 & $\mathrm{p}=0.094$ \\
\hline & 4 & $5 ; 12$ & 1.5 & $\mathrm{p}=0.267$ \\
\hline & 8 & $5 ; 13$ & 2.8 & $\mathrm{p}=0.063$ \\
\hline & 12 & $5 ; 11$ & 2.7 & $\mathrm{p}=0.072$ \\
\hline Total $\mathrm{P}_{\text {Gross }}$ & 1 & $4 ; 12$ & 5.9 & $\mathrm{p}=0.007$ \\
\hline & 2 & $4 ; 12$ & 2.5 & $\mathrm{p}=0.098$ \\
\hline & 4 & $4 ; 10$ & 2.4 & $\mathrm{p}=0.115$ \\
\hline & 8 & $4 ; 14$ & 0.8 & $\mathrm{p}=0.543$ \\
\hline & 12 & $4 ; 12$ & 4.2 & $\mathrm{p}=0.024$ \\
\hline $\mathrm{R}_{\mathrm{s}}$ & 1 & $5 ; 15$ & 1.8 & $\mathrm{p}=0.176$ \\
\hline & 2 & $5 ; 12$ & 2.6 & $\mathrm{p}=0.085$ \\
\hline & 4 & $5 ; 11$ & 16.9 & $\mathrm{p}=0.0001$ \\
\hline & 8 & $5 ; 14$ & 5.1 & $\mathrm{p}=0.007$ \\
\hline & 12 & $5 ; 10$ & 4.7 & $\mathrm{p}=0.018$ \\
\hline $\mathrm{R}_{\mathrm{z}}$ & 1 & $5 ; 15$ & 6.5 & $\mathrm{p}=0.002$ \\
\hline & 2 & $5 ; 13$ & 2.0 & $\mathrm{p}=0.139$ \\
\hline & 4 & $5 ; 14$ & 6.1 & $\mathrm{p}=0.003$ \\
\hline & 8 & $5 ; 14$ & 2.6 & $\mathrm{p}=0.073$ \\
\hline & 12 & $5 ; 14$ & 8.7 & $\mathrm{p}=0.001$ \\
\hline $\mathrm{T}$ & 1 & $5 ; 15$ & 3.8 & $\mathrm{p}=0.021$ \\
\hline & 2 & $5 ; 15$ & 0.4 & $\mathrm{p}=0.825$ \\
\hline & 4 & $5 ; 16$ & 2.1 & $\mathrm{p}=0.120$ \\
\hline & 8 & $5 ; 16$ & 1.8 & $\mathrm{p}=0.177$ \\
\hline & 12 & $5 ; 14$ & 3.7 & $\mathrm{p}=0.023$ \\
\hline & & & & \\
\hline & & & \\
& & & &
\end{tabular}

\subsection{Discussion}

This is the first study to measure the influence of symbiont diversity and hostsymbiont specificity on the autotrophic potential of novel cnidarian-dinoflagellate symbioses. It demonstrates that homologous (both cultured and freshly-isolated) Symbiodinium cells $A$. pulchella reach a stable state sooner than do heterologous 
Chapter 2

Symbiodinium types when infected into A. pulchella. Taking up homologous Symbiodinium cells also allows the host to achieve a fully autotrophic state at an earlier stage than when heterologous Symbiodinium types are taken up. Moreover, a marked trend was eveient towards the host anemones containing Symbiodinium B1 (both homologous and heterologous) benefiting most from their symbiotic partners. Of note, A. pulchella heterologous Symbiodinium B1was also successful, emphasising the specificity of $A$. pulchella for this symbiont type. The possible reasons for these differential patterns and their potential ecological significance will be considered here.

\subsubsection{Infection dynamics}

The finding of a peak in symbiont cell division just after dawn, as demonstrated by most of the Symbiodinium types, is supported by previous studies (Fitt and Trench, 1983; Fitt et al., 2000) and is a common characteristic of dinoflagellate growth (Chisholm, 1981). The delayed peak in the division at 10am of the cultured homologous symbionts has, to our knowledge, no parallels in the literature, but suggests an innate difference between the symbiont types. The peak in algal division at Week 1 and the following decrease in division over the 12-week period indicate that, while there is a rapid re-population of the anemones by the symbionts, their growth becomes constrained as the density of symbiont cells increases. This could arise for a number of reasons: (1) space limitation within the host cell (Muscatine and Pool, 1979; Jones and Yellowlees, 1997); (2) increasing competition for resources such as inorganic nitrogen (Cook and D'Elia, 1987) or carbon (Weis, 1993; Davy and Cook, 2001b); or (3) host control of symbiont cell growth and mitosis by an unknown mechanism (McAuley, 1981; Douglas and Smith, 1984; Bossert and Dunn, 1986).

Given that both the freshly-isolated and cultured homologous algae were originally isolated from $A$. pulchella, this might allow for more rapid integration with the host (e.g. via intracellular communication across the membrane complex at the hostsymbiont interface; Wakefield and Kempf, 2001; Peng et al., 2010) and could explain their initially higher division rates during symbiosis establishment. The same 
may be true for Symbiodinium B1 which was originally isolated from A. pallida, a close relative of the Indo-Pacific host species used here. Similarly, most infectivity studies have shown that homologous symbiont types are readily accepted by their hosts, and that heterologous types are either rejected or display lower rates of population growth if accepted (Kinzie, 1974; Kinzie and Chee, 1979; Schoenberg and Trench, 1980b; Trench, 1987, 1997; Davy et al., 1997; Weis et al., 2001; BeldaBaillie et al., 2002; Rodriguez-Lanetty et al., 2003).

Despite the high initial division rates of the B1 types in this study, it was the heterologous Symbiodinium A1.4, E2 and F5.1 that attained the highest densities at Week 12. Symbiodinium E2 also displayed a higher density at Week 1 indicating that this type proliferated faster inside the host. This trend was different to that reported by Schoenberg and Trench (1980b), who found that homologous symbionts in Aiptasia tagetes (= pallida) reached and maintained higher densities than did heterologous symbionts. One interpretation of this trend is that these Symbiodinium types had overshot the 'preferred' steady state for this symbiosis. Given that the B1 symbiont types divided more rapidly but reached lower densities at Week 12 than the heterologous types, indicates that they must have been expelled and/or degraded more rapidly. Cnidarians regulate their symbiont populations by controlling algal division rates, or by selectively destroying or expelling unwanted symbionts (Gates et al., 1992; Falkowski et al., 1993; Baghdasarian and Muscatine, 2000; Dunn et al., 2002; Dunn and Weis, 2009; Davy et al., 2012). It is conceivable, therefore, that the effectiveness of such control mechanisms was hindered in the symbioses with the heterologous types, though we know too little about the underlying cellular processes (Weis et al., 2008; Davy et al., 2012) to speculate further. Of note, the heterologous Symbiodinium B1 also attained a relatively high density, but this eventually declined to a level similar to that in the homologous symbioses. A similar overshoot and subsequent decline was observed in the temperate sea anemone Cereus pedunculatus when infected with its homologous symbionts (Davy et al., 1997). Far more research is required to unravel the complexities of host-symbiont biomass regulation in homologous and heterologous symbioses. 


\subsubsection{Photosynthesis and respiration}

The decrease in the cell specific rate of gross photosynthesis at high symbiont densities may be the result of limited resources such as carbon dioxide $\left(\mathrm{CO}_{2}\right)$ (Davy and Cook, 2001; Hoogenboom et al., 2010). Self-shading of the dinoflagellate cells may also occur at high densities (Jones and Yellowlees, 1997). In the case of Symbiodinium E2, however, gross photosynthesis remained constant despite increases in the cell density; the reason for this is unknown, but it may be that this Symbiodinium type, when in association with A. pulchella, requires more time to adjust to the in hospite environment and, as a result, the initial peak in photosynthesis was not observed.

Symbiodinium E2 and F5.1 had the highest cell densities and total rates of $\mathrm{P}_{\text {gross }}$ at Week 12 (though the values for type F5.1 were not significantly higher due to considerable variability). This relative photophysiological success of these two heterologous Symbiodinium types at first seems surprising, but it is important to note though that this higher total $\mathrm{P}_{\text {gross }}$ was countered by a relatively high $\mathrm{R}_{\mathrm{s}}$ rate and hence a lower P:R ratio. Photosynthetic stimulation of respiration has been noted previously in the sea anemone Anemonia viridis (Harland and Davies, 1995). Furthermore, the disproportionately higher rate of respiration $v s$ photosynthesis in these two heterologous symbioses may indicate that it is more energetically expensive for A. pulchella to maintain these Symbiodinium types than the others. Of particular note, Symbiodinium E2 was originally cultured from a single free-living cell collected from Wellington Harbour (New Zealand), which may explain its unusual behaviour when in hospite and why the symbiosis with this Symbiodinium type never reached a fully autotrophic state (i.e. a P:R of 1); there is increasing evidence that such free-living Symbiodinium types are common in the field (Takabayashi et al., 2012). In contrast, all of the other symbioses except for that with Symbiodinium F5.1 became fully autotrophic or near-autotrophic at some stage, though the point at which this occurred differed between types. This finding has implications for post-bleaching events, as those associations that reach a fully autotrophic state sooner will be more likely to recover. 


\subsubsection{Translocation}

Once a steady state had been reached, the percentage of carbon translocated ranged from 90 to $99.9 \%$ for the associations tested. The 'growth rate method' of estimating translocation has also been applied to numerous other symbioses, with similar estimates being derived (Muscatine et al., 1984; Steen and Muscatine, 1984; McCloskey et al., 1994; Day, 1994; Davy et al., 1996; Muller-Parker and Davy, 2001). However, only the association involving the cultured homologous Symbiodinium B1 displayed a significantly higher translocation rate at Week 12 than was seen in the other associations. This might suggest that, when in a stable symbiosis with its homologous symbiont, A. pulchella receives more translocated carbon than when in association with other symbiont types. However, the same pattern was not observed with freshly-isolated homologous symbionts, which grew faster and hence had less carbon available for translocation; this highlights the potentially different physiologies of cultured and freshly-isolated algae (Trench, 1971b; Domotor and D'Elia, 1984), which in this case was also apparent in the final CZAR values calculated for the homologous Symbiodinium cells.

Previous studies have demonstrated that different types of symbiotic dinoflagellates release different amounts of photosynthate and that they differentially influence incorporation patterns of translocated carbon in the host's tissues (Loram et al., 2007; Stat et al., 2008; Cantin et al., 2009). For example, Loram et al., (2007) found that when the sea anemone Condylactis gigantea hosted Symbiodinium clade A, a greater proportion of photosynthetically-fixed carbon was incorporated into animal lipids and amino acid pools than when conspecific anemones hosted clade B. Similarly, Cantin et al. (2009) showed that when the coral Acropora millepora harboured either Symbiodinium type C1 or D, it received more translocate from type C1. However, none of these studies attempted to measure the extent to which the photosynthate met the carbon demands of the host, and hence whether the observed differences had implications for host survival and proliferation. Moreover, further work is needed to identify the forms in which organic compounds are translocated in hospite and how these differ between Symbiodinium types (see review by Davy et al., 2012). 


\subsubsection{CZAR and ecological implications}

A number of past studies have examined the contribution of photosynthetically-fixed carbon to cnidarian host respiration (Muscatine et al., 1981; Davies, 1984; Muscatine et al., 1984; Steen and Muscatine, 1984; Edmunds and Davies, 1986 and 1989; Smith and Muscatine, 1986; Farrant et al., 1987a and b; Davies, 1991; Davy et al., 1996; Verde and McCloskey, 1996a and b; Tremblay et al., 2012), however this is the first study to compare this parameter between symbioses involving the same host species but different symbiont types. The range of CZAR values obtained in this study is comparable to previous reports. Studies using the "growth rate method" (Muscatine et al., 1981) in sea anemones, as well other cnidarians such as jellyfish and zoanthids, estimated the CZAR to generally be $>90 \%$ and sometimes $>100 \%$ under wellilluminated conditions (Steen and Muscatine, 1984; McCloskey et al., 1994; Day, 1994; Davy et al., 1996). However, given the number of unsubstantiated assumptions involved in this method (e.g. that host /symbiont respiration rates are proportional to their protein ratio), the CZAR values obtained may well be over-estimates (Muscatine et al., 1981). Indeed, in the coral Stylophora pistillata, the more direct approach of measuring translocation by ${ }^{14} \mathrm{C}$-radiolabelling yielded a much lower CZAR value than that derived via the growth rate method (40\% vs. 90\%; Muscatine et al., 1984), though this approach too has its shortcomings(for detailed discussion see Davy et al., 1996, 2012; Muller-Parker and Davy, 2001). Nevertheless, as a comparative tool, the growth rate method provides a valuable means of examining the relative performance of different symbiont types.

The trend of an increased CZAR over the experimental period for all the associations, suggests that more carbon becomes available to the host once the symbiosis reaches a stable state. This is of course not surprising given the considerable increase in the density of symbionts during symbiosis establishment; it was not the result of a corresponding decrease in animal respiration as no significant decrease in respiration was noted for any of the associations. The difference in photosynthetic performance between symbiont types has been widely studied (Rowan, 2004; Goulet et al., 2005;

Berkelmans and van Oppen, 2006; Cantin et al., 2009) and is very relevant when assessing how a particular host-symbiont combination will fare both during and after 
a bleaching event. However, it is not simply the photophysiology of the symbiont that will determine the long-term success of an association but more importantly the amount of carbon that is made available to the host when in symbiosis with a specific symbiont type and the metabolic demands of the host (Muscatine et al., 1981; Cantin et al., 2009). In the current study, while there were no significant differences in the CZAR between the symbioses tested, there was a marked trend towards the host anemones containing Symbiodinium B1 (both homologous and heterologous) benefiting most from their symbiotic partners. Greater replication would likely have clarified this trend further, however the difficulty in generating large numbers of aposymbiotic anemones made obtaining more replicates problematic. Nevertheless, the high CZAR values obtained for the symbioses with Symbiodinium B1, along with significantly higher Week-12 P:R ratios of these symbioses, suggest that anemones containing Symbiodinium B1 would likely grow faster and exhibit greater survivorship than those containing the other symbiont types. Similarly, Little et al. (2004) demonstrated that juveniles of the corals Acropora tenuis and Acropora millepora grow 2-3 faster when in symbiosis with Symbiodinium clade C than with clade D.

This study indicates that hosts with different-symbiont types have different photosynthetic and respiratory attributes, and that these impact the autotrophic potential of the symbiosis. This is demonstrated by the earlier onset of full autotrophy and the higher final CZAR values in the symbioses hosting Symbiodinium B1 (both homologous and heterologous). Indeed, our data highlights the functional diversity present amongst associations hosting different symbiont types. Future work is needed to determine whether the innate photosynthetic capacity of the symbiont or the influence of host-symbiont interplay on photosynthetic performance is more important for the onset and persistence of novel cnidarian-dinoflagellate symbioses. Similarly important is elucidation of the symbiosome membrane complex, across which all nutritional interplay must occur, and how its function is influenced by different host-symbiont pairings. Furthermore, it will be important to extend this type of work to corals in the field. Indeed, it should be noted that although some corals are capable of acquiring symbionts from the water column, this acquisition is temporary as these infections are not maintained as stable symbioses (Coffroth et al., 2010). 
Chapter 2

However, there is evidence for post-bleaching shifts in the dominant member of a mixed symbiont population (Baker, 2001; Baker et al., 2004; Berkelmans and van Oppen, 2006; Mieog et al., 2007; Jones et al., 2008) thus the differential performance of different symbiont types may still have a profound impact on the growth and survival of the host. 


\section{Chapter 3}

\section{The influence of symbiont type on host cell $\mathrm{pH}$ and physiology of a novel cnidarian-algal association}

\subsection{Introduction}

Cnidarian-dinoflagellate symbioses are the foundation of coral reefs, which in turn support a wide diversity of other organisms (Linden et al., 2002; Furla et al., 2005). The dinoflagellate symbionts (genus Symbiodinium) contribute substantially to the productivity, survival and success of their coral hosts (LaJeunesse, 2002) by providing photosynthetic carbon (Muscatine et al., 1981; Davies, 1984), promoting the recycling and conservation of essential nutrients (Wang and Douglas, 1998), and by enhancing rates of calcification (Moya et al., 2008). However, despite the immense ecological importance of this symbiosis, little is still known about the cell biology that underlies symbiosis establishment, maintenance and dysfunction (Weis et al., 2008; Yellowlees et al., 2008; Weis and Allemand, 2009; Davy et al., 2012).

Intracellular $\mathrm{pH}(\mathrm{pHi})$ influences various facets of cell metabolism. The functioning of membrane channels and enzymes, intracellular signalling and the availability of ions are all modulated by $\mathrm{pHi}$, so it is imperative that changes in $\mathrm{pHi}$ are minimized and tightly regulated (Busa and Nucitelli, 1984; Busa, 1986; Venn et al., 2009). pHi is therefore likely to play an important role in the cnidarian-dinoflagellate symbiosis, especially with respect to inter-partner signalling and transport across the symbiosome membrane and vacuolar space that separate the symbiont from its host (Wakefield and Kempf, 2001). For example, pHi influences the speciation of dissolved inorganic carbon (DIC), whose transport into the dinoflagellate symbionts is required for photosynthesis (Allemand et al., 1998; Leggat et al., 1999), as well as the speciation of inorganic nitrogen that is essential for growth (Miller and Yellowlees, 1989). pHi is also known to play a role in the regulation of the cell growth cycle in some organisms and could provide a mechanism by which host 
cnidarians regulate cell division by their symbionts (Davy et al., 2012). Despite the obvious importance of $\mathrm{pHi}$, however, we know very little about this parameter in the cnidarian-dinoflagellate symbiosis, largely due to methodological limitations that are only just being overcome. Crucially, however, Venn et al. (2009) recently used a pHsensitive fluorescent probe and confocal microscopy to measure pHi in host cells of the symbiotic anemone Anemonia viridis and the coral Stylophora pistillata. They found that host $\mathrm{pHi}$ was about $7.13 \pm 0.24$ in the dark and $7.41 \pm 0.22$ in the light for S. pistillata and $7.01 \pm 0.27$ in the dark and $7.29 \pm 0.15$ in the light for A. viridis. This important finding highlights the link between pHi of the host cell and the photosynthetic activity of its symbionts, and raises questions about how the differential photosynthetic performance of various symbiont types might influence $\mathrm{pHi}$ of the host cell cytoplasm. Furthermore, it highlights the need to test the generality of Venn et al. (2009)'s findings and whether the optimal $\mathrm{pH}$ of different symbiont types falls within the range of host $\mathrm{pHi}$ values measured. This study addressed these issues in the sea anemone Aiptasia pulchella, a widely employed model system for coral research (Weis et al., 2008). Figure 3.1 demonstrates four possible scenarios that depict how the total photosynthetic and respiration rates of the symbiont may affect the pHi of the host cell thus indicating the influence that different symbiont types may have on the intracellular physiology of the host. The aim of the study was to determine if $\mathrm{pHi}$ was a reflection of symbiont type and whether the optimal $\mathrm{pH}$ for photosynthesis coincides with $\mathrm{pHi}$. In order to answer these questions, aposymbiotic (i.e. symbiont-free) sea anemones were infected with a range of Symbiodinium types and: (1) intact symbiosomes (i.e. the vacuoles that house the symbionts) were isolated, exposed to a range of ambient pHs, and photosynthetic performance of the symbiotic dinoflagellates measured; and (2) the pHi of the host cell was measured. 

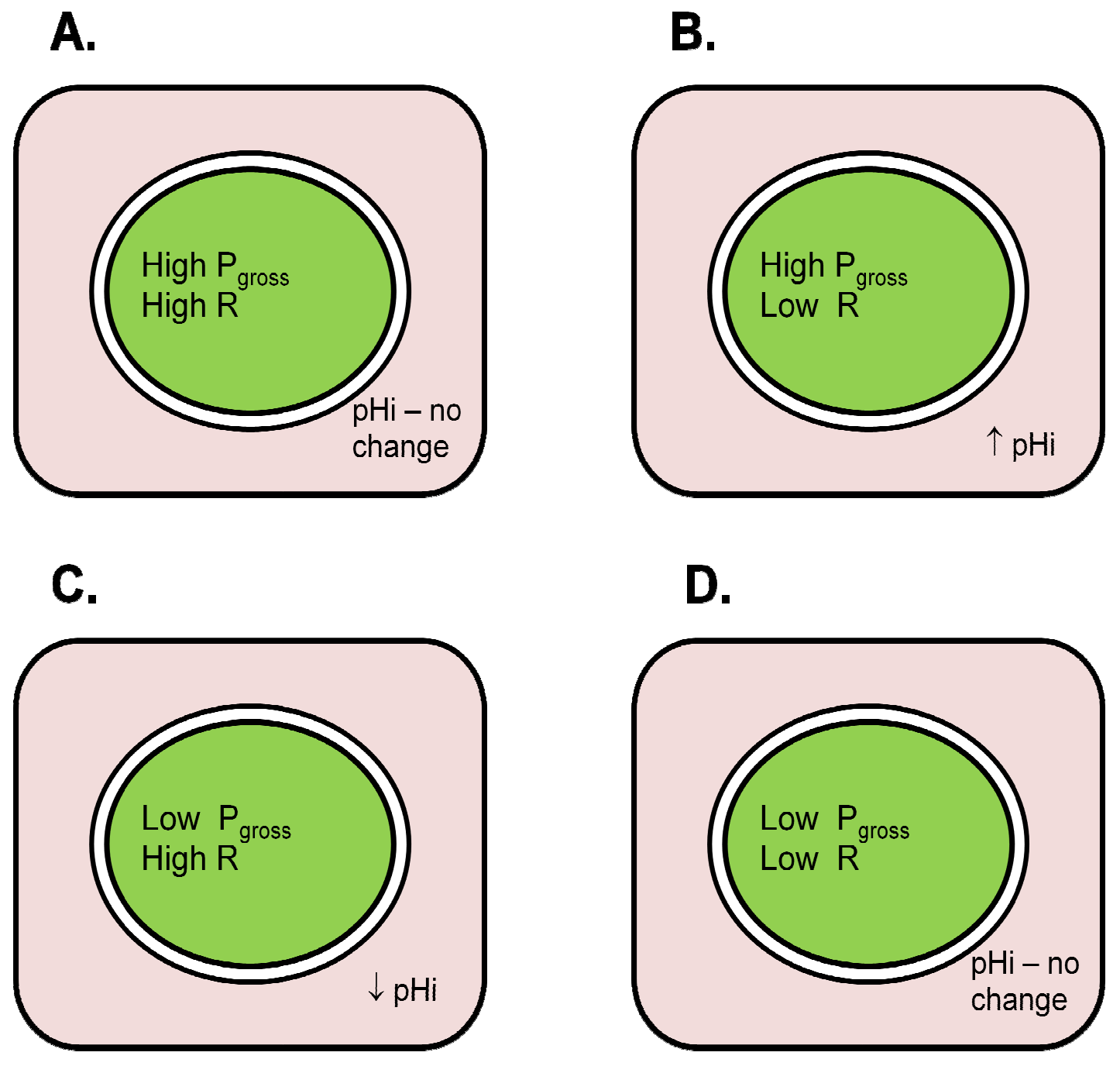

Figure 3.1. A conceptual diagram illustrating the potential relationship between photosynthetic and respiratory activity and internal host $\mathrm{pH}$ (pHi). Four scenarios are presented each representing a cnidarian host cell and its symbiont surrounded by the symbiosome membrane: A) High levels of photosynthesis and respiration balance each other out resulting in no change in the pHi. B) If levels of photosynthesis exceed respiration an increase in pHi is expected. C) If levels of respiration exceed photosynthesis a decrease in pHi is expected. D) Low levels of photosynthesis and respiration balance each other out resulting in no change in $\mathrm{pHi}$. 
Chapter 3

\subsection{Materials and methods}

The sea anemone Aiptasia pulchella was used as the experimental host and infected with different Symbiodinium types (cultured Symbiodinium B1, heterologous Symbiodinium B1 as well as Symbiodinium E2). After 12 weeks intact symbiosomes were isolated from the infected anemones, exposed to a range of ambient $\mathrm{pHs}$ in ordre to simulate a change in host cell $\mathrm{pHi}$, and the photosynthetic performance of the symbiotic dinoflagellates was measured. Host cells were also isolated from the anemones and the pHi of the host cell was measured using confocal microscopy.

\subsubsection{Experimental organisms}

The sea anemone, A. pulchella (originally from the Indo-Pacific region), was cultured at $25{ }^{\circ} \mathrm{C}$ and an irradiance of $100 \mu \mathrm{mol}$ photons $\mathrm{m}^{-2} \mathrm{~s}^{-1}$ (12 h light: $12 \mathrm{~h}$ dark) for a period of $>1$ year prior to use. The anemones were fed twice weekly with freshly hatched Artemia sp. To render the anemones aposymbiotic (i.e. symbiontfree), 120 individuals (oral disc diameter $\sim 50 \mathrm{~mm}$ ) were placed in six 1-L lightproof containers (20 individuals in each) filled with $0.44 \mu \mathrm{m}$ filtered seawater (FSW) and cold shocked at $4{ }^{\circ} \mathrm{C}$ for 24 hours. The cold-shock process was repeated at weekly intervals for five weeks and the feeding regime was retained between coldshocks, when care was taken to keep the anemones in low light $\left(<1 \mu \mathrm{mol}\right.$ photons $\mathrm{m}^{-}$

$2 \mathrm{~s}^{-1}$ ). The aposymbiotic anemones were maintained in the dark at $25{ }^{\circ} \mathrm{C}$ for approximately six months, when their aposymbiotic state was confirmed by exposure to a daily light cycle (12 h light:12 h dark) at an irradiance of $100 \mu \mathrm{mol}$ photons $\mathrm{m}^{-2}$ $\mathrm{s}^{-1}$ for a four-week period; fluorescence microscopy (Olympus Provis AX70 microscope using autofluorescence) at $100 \times$ magnification was also used to screen for the presence of symbionts. Only anemones deemed to be completely free of their symbionts were used for subsequent experiments.

Two heterologous Symbiodinium cultures (i.e. from hosts other than the A. pulchella culture used here), as well as a culture of the homologous type (sub-clade B1), were grown at $25^{\circ} \mathrm{C}$ and an irradiance of $100 \mu \mathrm{mol}$ photons $\mathrm{m}^{-2} \mathrm{~s}^{-1}$ on a light regime of 12 $\mathrm{h}$ light: $12 \mathrm{~h}$ dark. The cultures were grown in silica-free $\mathrm{f} 2$ medium at $\mathrm{pH} 8$ (Sigma- 
Chapter 3

Aldrich, Auckland, New Zealand) over a six-week period prior to use in the infection study. The heterologous Symbiodinium sub-clades were Symbiodinium E2 (culture ID CCMP421), originally collected as a free-living alga from Wellington Harbour, New Zealand, and a heterologous Symbiodinium B1 (culture ID F1Ap2), originally isolated from A. pallida, Long Key, Florida. The former of these types was selected because, in previous studies, its photosynthetic performance in symbiosis with the lab culture of $A$. pulchella had been particularly poor (see Chapter 2). The heterologous B1 allowed for a direct comparison between the same symbiont type but from different (though closely related) host species/geographic origins.

Symbiodinium types $(\mathrm{n}=3$ samples per culture or isolate) were genetically identified prior to infection (see Appendix 3 for the ITS2 sequences). DNA was extracted from zooxanthellae as described by Logan et al. (2010). PCR was performed using the following Symbiodinium-specific ITS2 primers: forward primer (ITSD), 5'-GTG AAT TGC AGA ACT CCG TG-3'; reverse primer (ITS2rev2), 5'-CCT CCG CTT ACT TAT ATG CTT-3'. Reactions were performed in a total volume of $30 \mu \mathrm{L}$ using the MyTaq ${ }^{\mathrm{TM}}$ Mix with an amplification profile consisting of 1 min $95{ }^{\circ} \mathrm{C}, 35$ cycles of 1 min $95{ }^{\circ} \mathrm{C}, 15$ s $55{ }^{\circ} \mathrm{C}, 10 \mathrm{~s} 72{ }^{\circ} \mathrm{C}$, and a final hold temperature of $4{ }^{\circ} \mathrm{C}$. Single strand conformation polymorphism (SSCP) was performed on all PCR products. PCR products were sequenced by Macrogen Inc. (Seoul, Korea). Sequences were aligned using the ClustalW plugin in Geneious Pro 4.8.5 and then edited. A blast search was then performed in the NCBI database in order to identify the sequences (see Table A3.1).

\subsubsection{Infection of aposymbiotic $A$. pulchella with different Symbiodinium types}

Aposymbiotic specimens of $A$. pulchella $(\mathrm{n}=44$ for each symbiont treatment for the respirometry experiments and $n=10$ per treatment for the confocal fluorescence microscopy work) were infected with cultured Symbiodinium cells by pipetting a concentrated drop of the dinoflagellate suspension (approximately 1 million cells $\mathrm{mL}^{-1}$ ) onto the oral disc of each anemone, with a sharp-ended glass pipette. A drop of one-day old Artemia sp. culture was then pipetted onto the oral disc to induce a feeding response and encourage the uptake of the Symbiodinium cells, as described 
Chapter 3

by Davy et al. (1997). Anemones were kept in FSW in $20 \mathrm{~mL}$ glass containers that received a water change 8 hours after infection and were maintained as before.

After 12 weeks, representative anemones (3 individuals per treatment) were selected from the pools of anemones reinfected with each of the different Symbiodinium types, to confirm symbiont identity. Individual anemones were homogenized in a hand-held, glass tissue grinder for approximately $5 \mathrm{~min}$. The homogenate was then centrifuged at $\times 400 \mathrm{~g}$ for $5 \mathrm{~min}$ to separate the animal and algal fractions. DNA extraction and genetic typing were performed as described previously. All treatments were found to harbour the same Symbiodinium type as the one introduced at the start of the experiment (see Appendix 3 for the ITS2 sequences).

\subsubsection{Symbiosome isolation and verification}

Four anemones from each Symbiodinium type and $\mathrm{pH}$ treatment were homogenized in $1 \mathrm{~mL}$ of FSW in a tissue grinder and centrifuged at $\times 400 \mathrm{~g}$. The supernatant was removed and the process repeated twice. The pellet was then passed through a stepwise sucrose gradient $(30 \%-80 \%)$ by centrifugation at $\times 3893 \mathrm{~g}$ for 1 hour (Figure 3.2). The $40 \%$ sucrose fraction (the symbiosome-enriched fraction) was collected fot further experiments. Three samples per symbiont treatment were also collected from each isolation and stained for $10 \mathrm{~min}$ with the FM1-43 lipophilic fluorescent probe $(30 \mu \mathrm{M})$ as well as $10 \mu \mathrm{g} \mathrm{mL}^{-1}$ Hoechst 33342 for visualisation of the symbiosome membrane and nuclear material respectively; this enabled identification of the symbiosome membrane (Kazandjian et al., 2008). The presence of symbiosomes was confirmed with fluorescence microscopy (Olympus Provis AX70 microscope) at $100 \times$ magnification using a fluorescein isothiocyanate (FITC) filter to visualise FM 1-43 fluorescence and a 6-diamidino-2-phenylindole dihydrochloride, 2-(4amidinophenyl)-6-indolecarbamidine dihydrochloride (DAPI) filter to visualise Hoechst 33342 fluorescence. When examining the symbiosome-enriched fraction, all cells were noted to possess the surrounding symbiosome membrane. Dyes used for microscopy were purchased from Invitrogen (Auckland); other chemicals were purchased from Sigma-Aldrich (Auckland). 


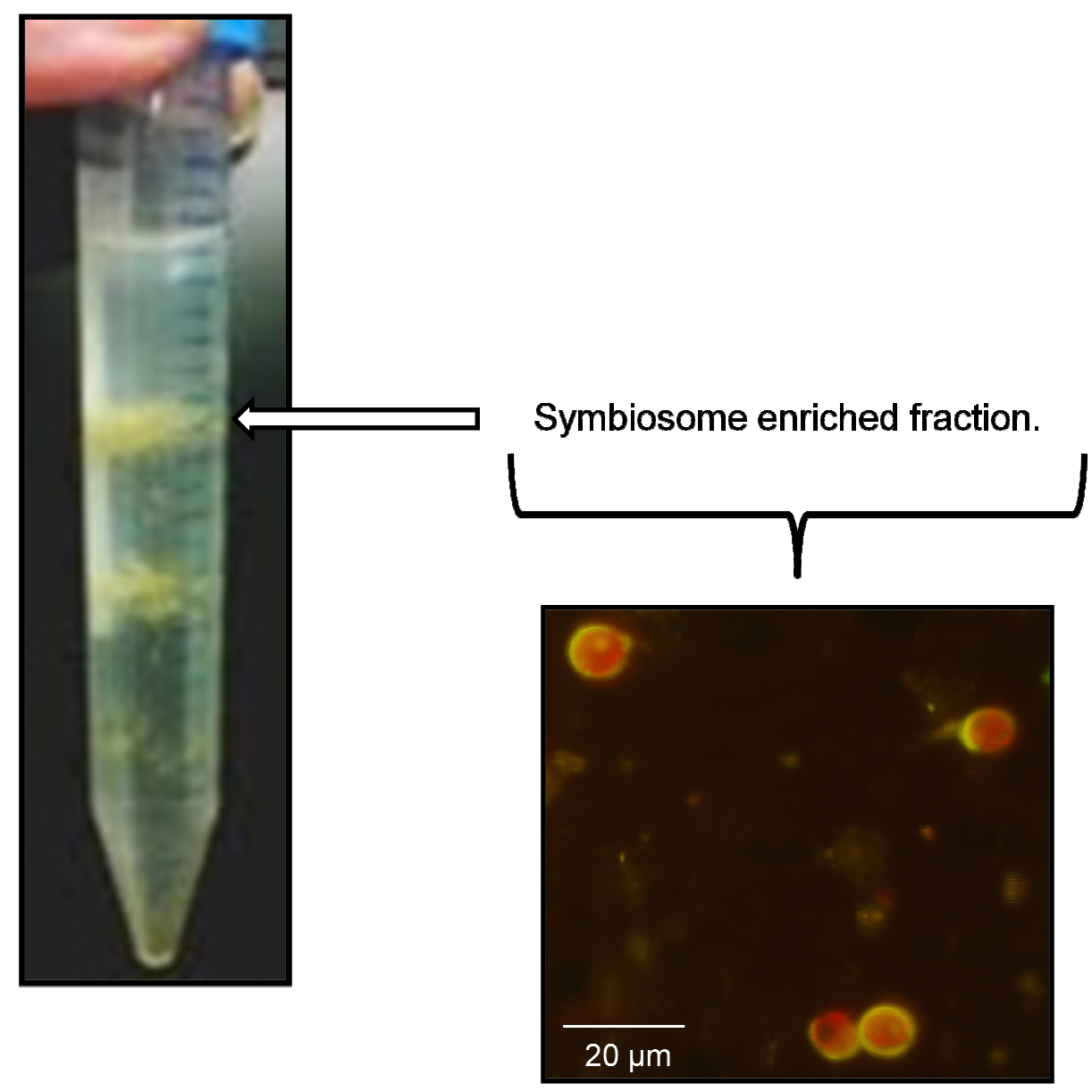

Figure 3.2. Photograph of the step-wise sucrose gradient depicting the $40 \%$ sucrose layer containing the symbiosome enriched fraction and the fluorescence microscope image of the symbiosome enriched fraction showing the symbiosome membrane (golden layer) after staining with FM1-43.

\subsection{4. $\mathrm{pH}$ manipulation and determination of photosynthetic rates}

The enriched symbiosome fractions isolated from individual anemones for each treatment were placed in a $10 \mathrm{~mL}$ glass chamber, itself situated in a glass water bath set at $25{ }^{\circ} \mathrm{C}$ (symbiosome fractions were isolated individually from 4 anemones per each $\mathrm{pH}$ and symbiont treatment). All the anemones used were for the isolations were of similar size. The density of the symbiosomes isolated from each anemone ranged from $1.5 \times 10^{5}$ to $3.2 \times 10^{6}$ cells $\mathrm{mg}^{-1}$ protein with differences in density arising from the variable infection success of the different symbiont types. The chamber contained FSW adjusted to a specific $\mathrm{pH}$, ranging from 6 to 8.5 (in $0.25 \mathrm{pH}$ 
Chapter 3

unit increments). The $\mathrm{pH}$ was adjusted using $2 \mathrm{M} \mathrm{HCl}$ and $5 \mathrm{M} \mathrm{NaOH}$. A pilot study performed using anemones with a comparable symbiosome density showed that the $\mathrm{pH}$ remained stable over the course of the experiment. Unpublished data indicated that FSW buffered with PBS required a comparable volume of $2 \mathrm{M} \mathrm{HCl}$ and $5 \mathrm{M}$ $\mathrm{NaOH}$ to adjust the $\mathrm{pH}$ to the desired increments, so buffering was determined to have no effect on the ion concentration and was deemed unnecessary. The cells were kept in suspension by gently stirring with a magnetic spin bar. The chamber was sealed by a glass lid with a rubber O-ring, into which an oxygen electrode (FIBOX 3 - fiber-optic oxygen meter, PreSens GmbH, Germany) and temperature probe were inserted; these were connected to the oxygen meter (see Figure 3.3 for experimental set-up). The chamber was illuminated by a Thorn38 PAR (photosynthetically active radiation) $150 \mathrm{~W}$ sealed beam-reflector lamp and irradiance incident on the surface of the chamber was measured by a QSL-100 irradiance meter (Biospherical Instruments Inc.). Maximum photosynthetic and dark respiratory $\mathrm{O}_{2}$ fluxes were measured at each $\mathrm{pH}$ for symbiosomes isolated from all three associations. The respiration rate $\left(\mathrm{mL} \mathrm{O}_{2} \mathrm{~h}^{-1}\right)$ of each the different symbiosome suspensions was measured in darkness for about an hour or until a constant rate was observed. The oxygen tension was not allowed to fall below 50\% saturation (Davies, 1984). The PAR lamp was then switched on and the rate of net photosynthesis by each symbiosome suspension was measured at $400 \mu \mathrm{mol}$ photons $\mathrm{m}^{-2} \mathrm{~s}^{-1}$ (>light-saturation for photosynthesis), again for about an hour or until the rate was constant. 


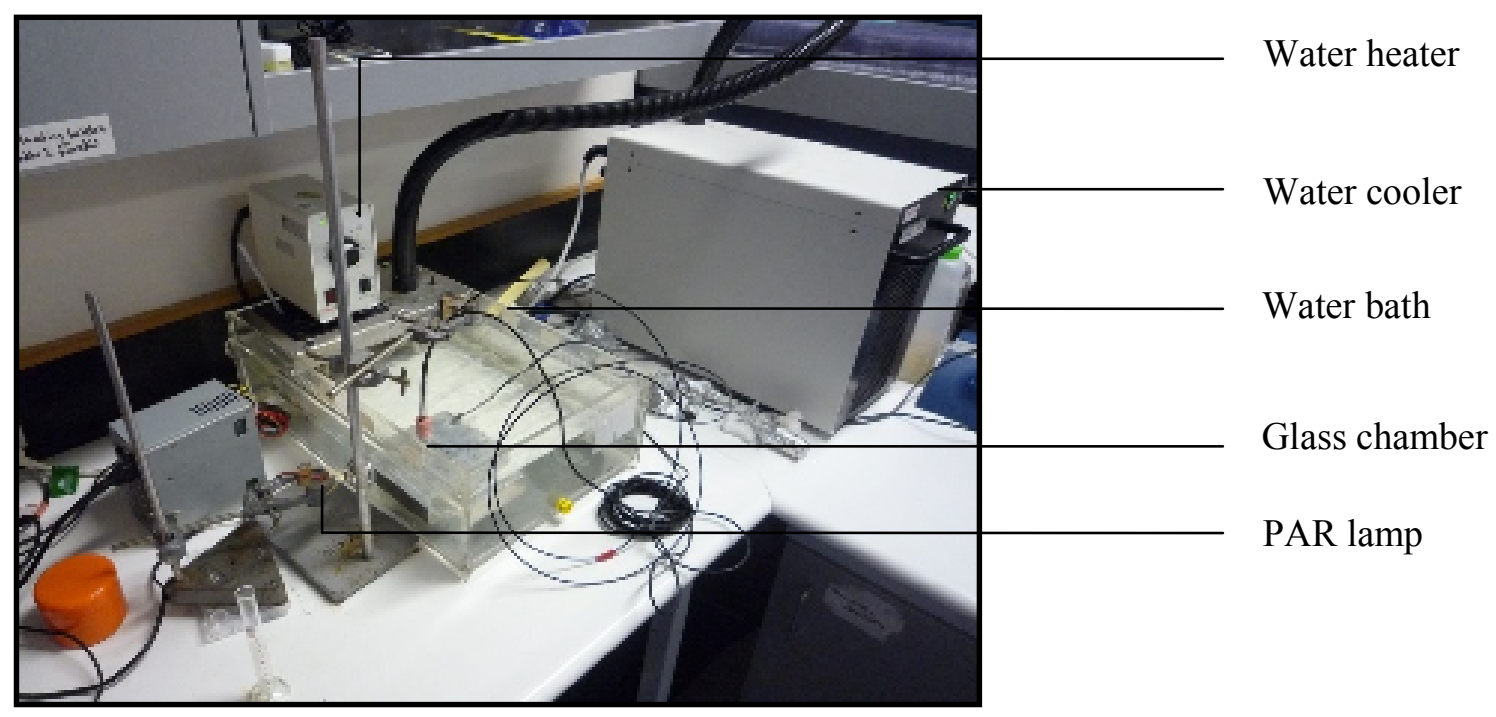

Figure 3.3. Photograph depicting the experimental $\mathrm{O}_{2}$ flux set-up used to measure the photosynthetic and respiration rates of the isolated symbiosomes at a range of $\mathrm{pH}$ from $\mathrm{pH} 6$ to $\mathrm{pH} 8.5$ (photograph taken by Anne Wietheger).

The rate of gross photosynthesis was calculated by adding net photosynthesis to dark respiration, and converted into carbon equivalents by assuming photosynthetic and respiratory quotients of 1.1 and 0.9, respectively (Muscatine et al., 1981, 1983; Muscatine, 1990). The contents of the chamber were collected and centrifuged at $\times 400 \mathrm{~g}$ for $5 \mathrm{~min}$ to collect the symbiosomes. This fraction was then resuspended in $1 \mathrm{~mL}$ FSW. Algal cells were counted using light microscopy at 400× magnification. The gross photosynthetic and respiration rates were adjusted for the respiration chamber volume and normalised to the average algal cell volume.

\subsubsection{A. pulchella cell isolation and staining}

A. pulchella host cells were isolated based on a protocol adapted from Gates and Muscatine (1992). Anemones were first incubated in calcium-free seawater for $60 \mathrm{~min}$. The tentacles from each anemone were then sliced off using a scalpel and cut into 1 to $2 \mathrm{~mm}$ sections. The sections were washed by transferring them from one glass slide to another, each with a drop of $3 \mathrm{U} \mathrm{mL}^{-1}$ lysozyme solution. This was repeated ten times to eliminate mucus. The chopped tentacles were then placed in a watch glass and the excess lysozyme solution pipetted off. A $300 \mu \mathrm{L} 0.05 \%$ trypsin 
Chapter 3

solution (Type III, Sigma Chemical Co.; in $\mathrm{Ca}^{2+}$-free FSW) was added to the tentacles. The watch glass was covered with parafilm to avoid evaporation and placed on an orbital shaker to allow the solution to agitate for $45 \mathrm{~min}$. The contents were then transferred to a polylysine coated plastic dish and the isolated cells were left to settle for $15 \mathrm{~min}$ in the dark. The excess enzyme solution was then poured off.

Three different probe solutions were prepared as follows: 1) host nuclei and mitochondria - a mixture of $5 \mu \mathrm{g} \mathrm{mL}^{-1}$ Hoechst 33342 and $5 \mu \mathrm{M}$ rhodamine; 2) host cell viability - a mixture of $10 \mu \mathrm{g} \mathrm{mL}^{-1}$ Hoechst 33342 and $10 \mu \mathrm{M}$ fluorescein diacetate; 3) $\mathrm{pHi}$ determination - a $10 \mu \mathrm{M}$ solution of the $\mathrm{pH}$ sensitive probe, carboxy SNARF-4f, 0.01\% Pluronic F-127, and 0.1\% dimethyl sulfoxide (DMSO). Cells were bathed in each solution in the dark at room temperature (approx. $22{ }^{\circ} \mathrm{C}$ ) for 10 min with the exception of the Hoechst 33342 and fluorescein diacetate mix, which required $20 \mathrm{~min}$. After dye loading, cells were washed with $200 \mu \mathrm{L} \mathrm{Ca}^{2+}$ free FSW. Cells were treated with $200 \mu \mathrm{L} 1.5 \%$ carboxymethyl cellulose, so immobilizing them for confocal microscopy. All dyes used for microscopy were purchased from Invitrogen (Auckland); other chemicals were purchased from SigmaAldrich (Auckland).

\subsubsection{Host cell viability and pHi}

Isolated, stained host cells and their associated symbionts were visualized by confocal microscopy, conducted with an FV-1000 confocal microscope (Olympus, Auckland, New Zealand). All samples were imaged using $100 \times$ oil immersion lens, with excitation provided by a $405 \mathrm{~nm}$ laser for the Hoechst 33342 probe (emission at $460 \mathrm{~nm} \pm 10$ ), $473 \mathrm{~nm}$ laser for fluorescein diacetate and rhodamine (emission at $520 \mathrm{~nm} \pm 10$ and $530 \mathrm{~nm} \pm 10$, respectively), and a $635 \mathrm{~nm}$ laser for chlorophyll autofluorescence. Cells loaded with SNARF-4f (emission at 546 and $635 \mathrm{~nm}$ ) were excited at $473 \mathrm{~nm}$, with a scan speed of $400 \mathrm{htz}$ and the pinhole set at 1.51 units. Each cell was analyzed by capturing 10 frames in the $x / y$ plane, throughout the depth (z plane) of the cell to produce a z-stack profile. Images were processed using Olympus FV10-ASW 1.7 software and analysed using ImageJ software (http://imagej.nih.gov/ij/). In order to empirically determine the viability of the 
isolated cells, host cells were left to settle on the polylysine coated plates for up to 3 hours post-isolation, and were then stained with Hoechst and fluorescein at hourly intervals and visualized with confocal microscopy. Viable cells were identified every hour for 4 hours by green florescence of the host cell cytoplasm (as a result of the fluorescein probe) indicating an intact cell membrane. The percentage viability was determined from a count of 100 cells at 1,2,3 and 4 hours post host-cell isolation.

Measurements of $\mathrm{pHi}$ were performed by ratiometric analysis of SNARF-4f fluorescence (Venn et al., 2008). The ratio of fluorescence intensity of SNARF-4f at $546 / 635 \mathrm{~nm}$ was calibrated in vivo in the range $\mathrm{pH} 6-8.5$, by washing host endodermal cells in calibration buffer adjusted to the desired $\mathrm{pH}$ by the addition of hydrochloric acid $(\mathrm{HCl})$ or sodium hydroxide $(\mathrm{NaOH})$. The ionophore, nigericin, was added at $30 \mu \mathrm{M}$ to equilibrate $\mathrm{pHi}$ with the surrounding medium and eliminate $\mathrm{pH}$ gradients within the cell (Buckler and Vaughan-Jones, 1990). Calibration buffers contained the approximate cytosolic concentrations of major ions found in cnidarian cells (60 $\mathrm{mM} \mathrm{Na}^{+}, 200 \mathrm{mM} \mathrm{K}{ }^{+}, 190 \mathrm{mM} \mathrm{Cl}^{+}, 25 \mathrm{mM}$ Pipes (pH 6- 7.5) or Tricine (pH 8 - 8.5); Herrera et al., 1989; Goiran et al., 1997). Mannitol was added to adjust osmolarity to 1100 mosm $\mathrm{L}^{-1}$. The calibration curve is provided in Appendix 2 (Figure A2.1).

The $\mathrm{pH}_{\mathrm{i}}$ was then determined for dark-adapted host cells (after $20 \mathrm{~min}$ in the dark) as well as cells incubated for an hour under photosynthetically active radiation (PAR) that matched the conditions under which the anemones were cultured (100 $\mu \mathrm{mol}$ photons $\mathrm{m}^{-2} \mathrm{~s}^{-1}$ ). The cells were stained with SNARF-4f and visualized using confocal microscopy (as described previously). Only endodermal cells that contained two algae were used as they were most suitable for visualizing the $\mathrm{pHi}$ of the host-cell cytoplasm and were far more numerous than cells with three algae. After inspection of each cell in the $x, y$ and $z$ planes, SNARF-4f fluorescence intensity at 546 and $635 \mathrm{~nm}$ was recorded within a region of interest (ROI) selected in an area of host cytoplasm that did not overlap with any algal cells; only the area that showed no autofluorescence was selected. R was calculated after subtracting background fluorescence recorded in a second ROI in the medium surrounding the 
Chapter 3

cell. The 546/635 $\mathrm{nm}$ fluorescence intensity ratio (R) was related to $\mathrm{pH}$ by the following equation:

$\mathrm{pH}=\mathrm{pK}_{\mathrm{A}}-\log \left[\mathrm{R}-\mathrm{R}_{\mathrm{B}} / \mathrm{R}_{\mathrm{a}}-\mathrm{R} \times \mathrm{F}_{\mathrm{B}(\lambda 2)} / \mathrm{F}_{\mathrm{A}(\lambda 2)}\right]$

[Equation 2]

where (F) is fluorescence intensity measured at $635 \mathrm{~nm}(2)$ and the subscripts A and $B$ represent the limiting values at the acidic and basic end-points of the titration, respectively (i.e., $\mathrm{pH} 6$ and 8.5).

\subsubsection{Statistical analyses}

Data were tested for normality using the Kolmogorov-Smirnoff test and for homogeneity of variance using the Bartlett test. Multiple comparisons of R, $\mathrm{P}_{\text {gross }}$ and $\mathrm{pHi}$ between the different symbiotic associations and $\mathrm{pH}$ points were made using two-way analysis of variance $(\alpha=0.05)$, followed by LSD post hoc tests. A t-test was used to compare the pHi between the associations. PASW (Predictive Analytics SoftWare, Version 18) was used for all analyses.

\subsection{Results}

\subsubsection{Determination of host cell $\mathrm{pHi}$}

Images of Symbiodinium cells within their host cells, stained with various fluorescent probes and visualized with confocal microscopy, are shown in Figure 3.4. Following $20 \mathrm{~min}$ of dark exposure the pHi of the host cells was $6.9 \pm 0.04,6.7 \pm 0.14$ and $6.6 \pm 0.10$ in the associations with the homologous B1, heterologous B1 and E2 symbionts respectively. After $1 \mathrm{hr}$ of light exposure the $\mathrm{pH}$ increased to $7.0 \pm 0.04$, $6.9 \pm 0.08$ and $6.7 \pm 0.08$ respectively. Significant differences were noted between the dark- and light-adapted cells isolated from all the symbioses tested (T-test, $\mathrm{p}<$ 0.05 for all treatments). When comparing the pHi of the host cells isolated from the different associations, however, both after $20 \mathrm{~min}$ of darkness and after $1 \mathrm{hr}$ of light 


\section{Chapter 3}

exposure, there were no significant differences between any of the symbioses (oneway ANOVA, d.f. $=2 ; 40, \mathrm{~F}=1.2-1.9, \mathrm{p}>0.05)$.

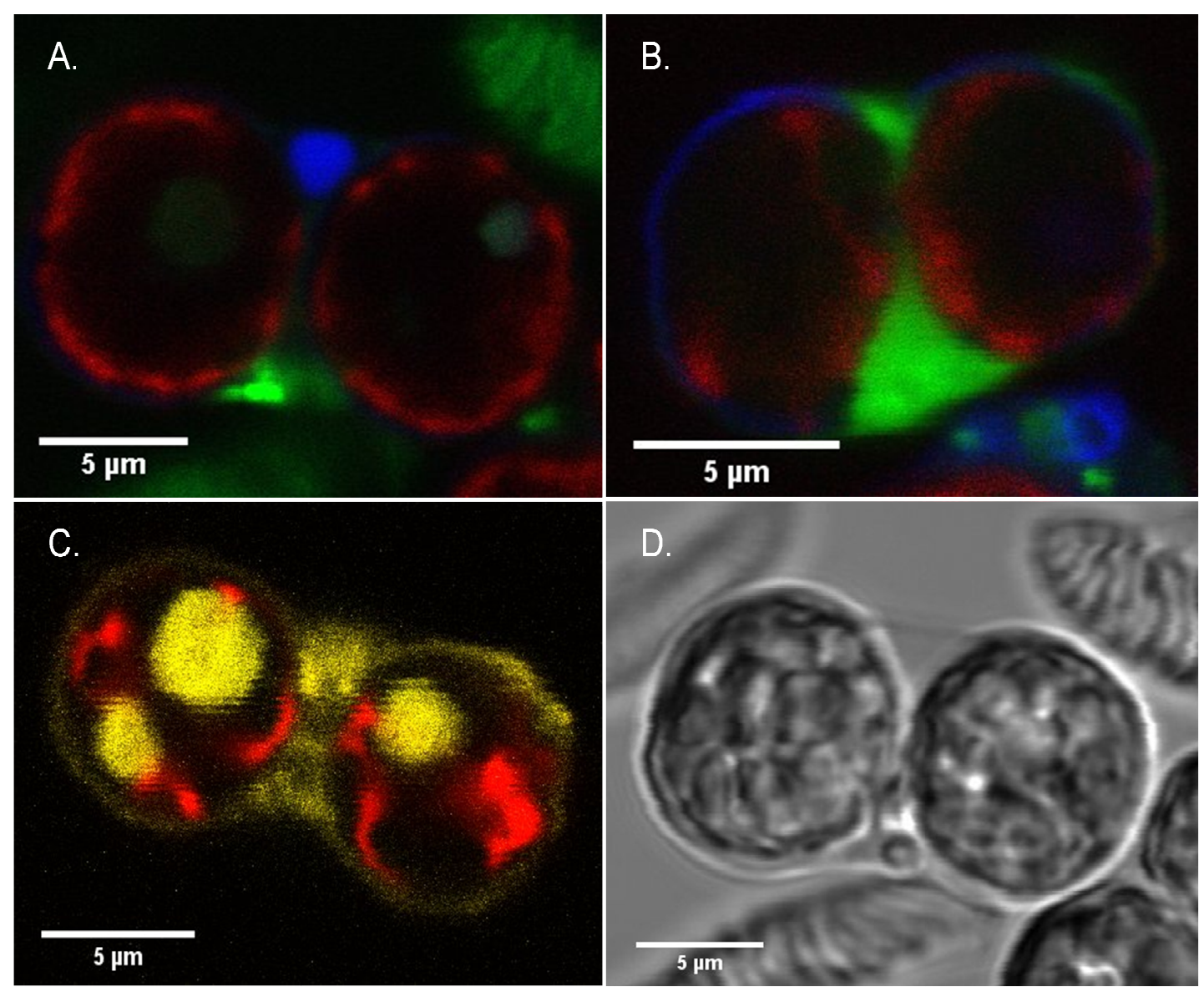

Figure 3.4. Cnidarian endodermal cells isolated from A. pulchella with two intracellular dinoflagellates. Confocal fluorescence microscope image of cells stained with (A) Hoechst 33342 and rhodamine 123, which stain nuclear material and mitochondria respectively. (B) Hoechst 33342 and fluorescein, which stain nuclear material and cytoplasm, respectively. (C) SNARF-4f for $\mathrm{pHi}$ determination. Colours represent the following: Red, autofluorescence of chlorophyll in intracellular algae; blue, nuclei stained with Hoechst 33342; green, mitochondria stained with rhodamine 123 (A) and cytoplasm stained with fluorescein (B); and yellow, cnidarian cytoplasm stained with SNARF-4f. (D) Light microscopy image. 
Chapter 3

\subsubsection{Effect of $\mathrm{pH}$ on gross photosynthetic and respiration rates}

Ambient $\mathrm{pH}$ had a significant effect on the rate of gross photosynthesis by the isolated symbiosomes. A positive interaction was observed between $\mathrm{pH}$ and symbiont type (two-way ANOVA, d.f. $=20, \mathrm{~F}=1.8, \mathrm{p}<0.05$ ). Significant differences in $\mathrm{P}_{\text {gross }}$ were observed both between different symbiont types and between different $\mathrm{pH}$ points (Figure 3.5A; two-way ANOVA, d.f. $=2, \mathrm{~F}=5.6, \mathrm{p}<$ 0.005 and two-way ANOVA, d.f. $=10, \mathrm{~F}=5.9, \mathrm{p}<0.005$ respectively). The symbiosomes isolated from the association infected with the homologous B1 type, displayed a significantly higher $\mathrm{P}_{\text {gross }}\left(0.0016 \mathrm{pg} \mathrm{C} \mathrm{cm}^{-3} \mathrm{~h}^{-1}\right)$ at $\mathrm{pH} 7.5$ than at any of the higher or lower $\mathrm{pH}$ values except for $\mathrm{pH} 8$ (two-way ANOVA with LSD post-hoc test, $\mathrm{p}<0.05$ for all comparisons); $\mathrm{P}_{\text {gross }}$ at $\mathrm{pH} 8$ was found to be significantly higher than the values at $\mathrm{pH} 7$ and below (two-way ANOVA with LSD post-hoc test, $\mathrm{p}<$ 0.05 for all comparisons). The symbiosomes isolated from the association with heterologous $\mathrm{B} 1$ displayed significantly higher $\mathrm{P}_{\text {gross }}$ at $\mathrm{pH} 6.75,7,7.25$ and 7.5 ( 0.0003 to $0.0008 \mathrm{pg} \mathrm{C} \mathrm{cm}^{-3} \mathrm{~h}^{-1}$ ) than the remaining $\mathrm{pH}$ values (two-way ANOVA with LSD post-hoc test, $\mathrm{p}<0.05$ for all comparisons) with the highest $\mathrm{P}_{\text {gross }}$ being achieved at pH 6.75 (two-way ANOVA with LSD post-hoc test, $\mathrm{p}<0.05$ ). The Symbiodinium E2 symbiosomes displayed the greatest $\mathrm{P}_{\text {gross }}$ at $\mathrm{pH} 8(0.0012 \mathrm{pg} \mathrm{C}$ $\mathrm{cm}^{-3} \mathrm{~h}^{-1}$ ), when it was significantly higher than the remaining $\mathrm{pH}$ values with the exception of $\mathrm{pH} 8.5$ (two-way ANOVA with LSD post-hoc test, $\mathrm{p}<0.05$ ). At a $\mathrm{pH}$ below 7 the Symbiodinium E2 symbiosomes displayed significantly reduced $\mathrm{P}_{\text {gross }}$ (two-way ANOVA with LSD post-hoc test, $\mathrm{p}<0.05$ for all comparisons).

With respect to the respiration rate, no interaction between $\mathrm{pH}$ and symbiont type was observed (Figure 3.5B; two-way ANOVA, d.f. $=20, \mathrm{~F}=1.2, \mathrm{p}>0.05$ ). When looking at the association with the heterologous B1 only however, ambient $\mathrm{pH}$ was found to have a significant effect on the respiration of the isolated symbiosomes (Figure 3.5B; one-way ANOVA, d.f. $=10 ; 22, \mathrm{~F}=5.1, \mathrm{p}<0.005$ ). The respiration rate at $\mathrm{pH} 7.75,8,8.25$ and 8.5 was significantly lower $\left(0.0003\right.$ to $0.0004 \mathrm{pg} \mathrm{C} \mathrm{cm}^{-3}$ $\mathrm{h}^{-1}$ ) than at the lower $\mathrm{pH}$ values with the exception of $\mathrm{pH} 7.25$ (one-way ANOVA with LSD post-hoc test, $\mathrm{p}<0.05$ for all comparisons). The $\mathrm{pH}$ at which the symbiosomes isolated from the different associations reached a total photosynthesis 
Chapter 3

to respiration ratio (P:R ratio) of 1 and above varied (Figure 3.6). The homologous $\mathrm{B} 1$ reached a P:R ratio of one and higher at $\mathrm{pH} 7.5,7.75$ and 8 , while the heterologous $\mathrm{B} 1$ did not reach a $\mathrm{P}: \mathrm{R}$ ratio of 1 but came close to it at $\mathrm{pH}$ 7-7.5. The symbiosomes with Symbiodinium E2 displayed a P:R ratio of 1 and greater from $\mathrm{pH}$ 8 upwards.
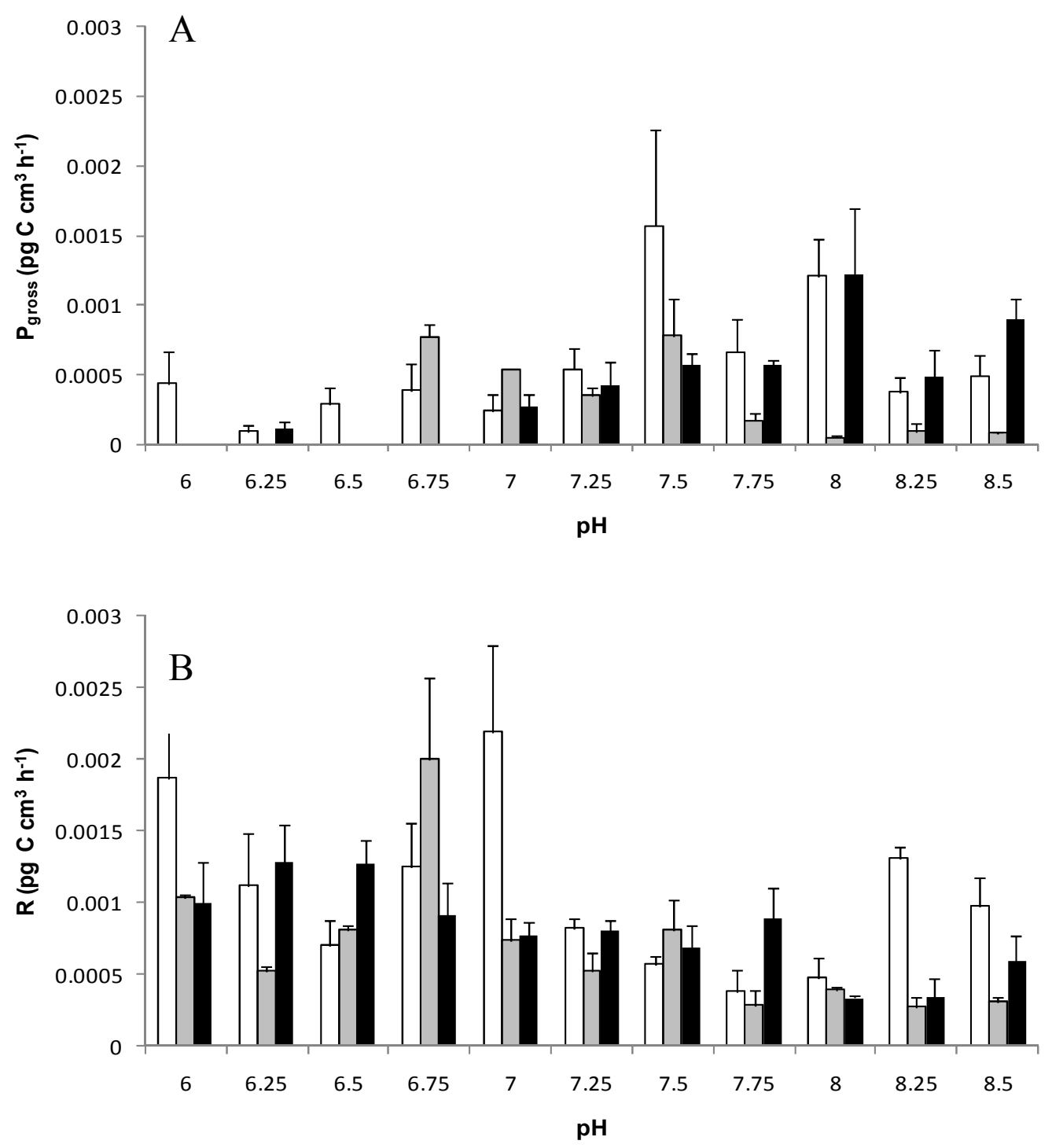

Figure 3.5. Relationship between gross photosynthetic rate $\left(\mathrm{P}_{\text {gross }} ; \mathrm{A}\right)$ and respiration rate $(\mathrm{R}$; B) of symbiosomes isolated from A. pulchella when in association with three different Symbiodinium types, and the $\mathrm{pH}$ of the medium in which they were suspended. The white, grey and black bars represent the homologous B1, heterologous B1 and subclade E2 Symbiodinium respectively. Values are means \pm SE, $n=4$. 


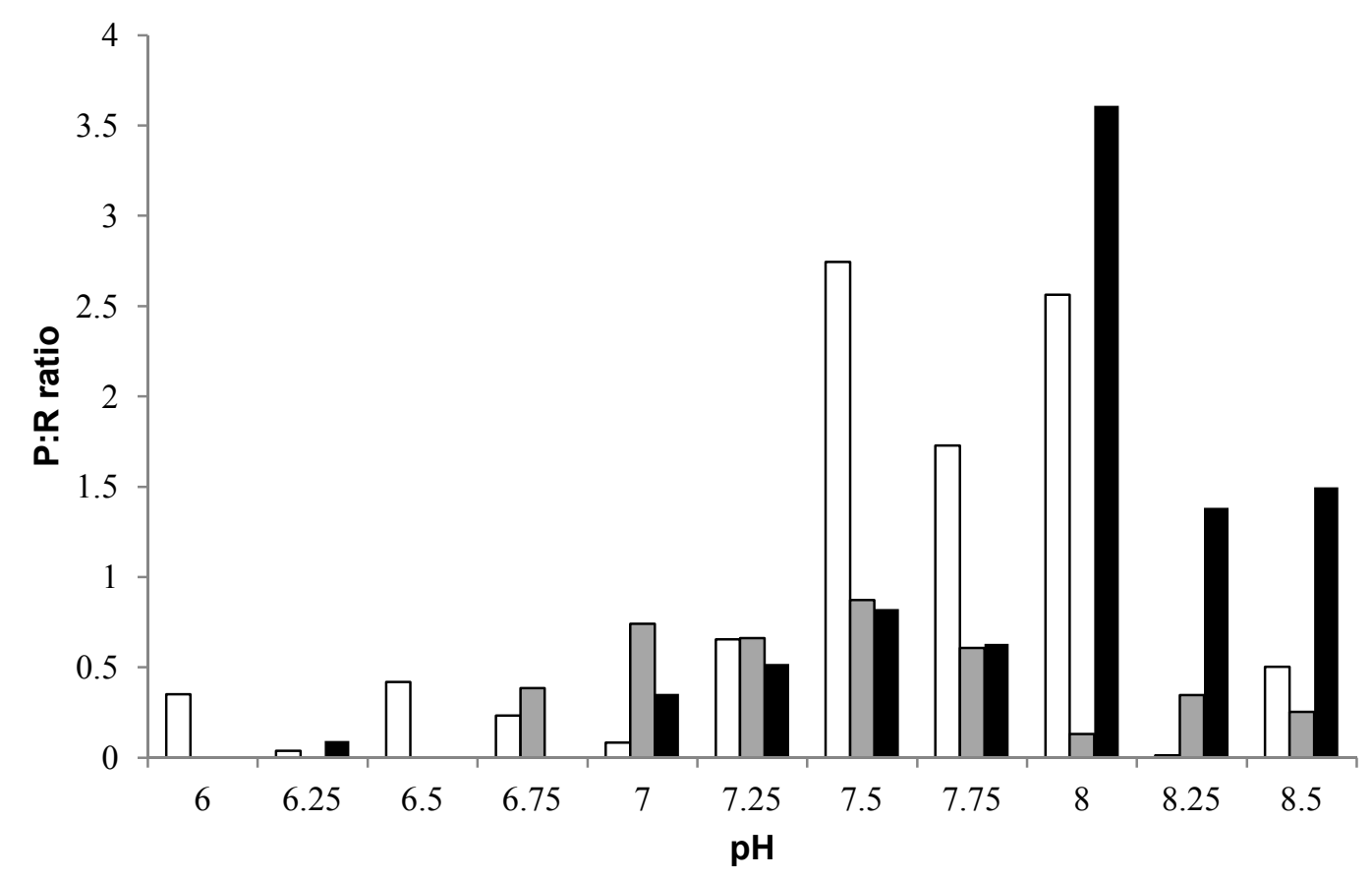

Figure 3.6. Relationship between the ratio of gross photosynthesis $\left(\mathrm{P}_{\text {gross }}\right)$ and respiration $(\mathrm{R})$ of symbiosomes isolated from $A$. pulchella when in association with three different Symbiodinium types, and the $\mathrm{pH}$ of the medium in which they were suspended. The white, grey and black bars represent the homologous B1, heterologous B1 and subclade E2 Symbiodinium respectively $(\mathrm{n}=4)$.

\subsection{Discussion}

This study reports the host-cell pHi in A. pulchella, an important model organism in symbiosis research (Weis et al., 2008). More specifically, this study is the first to measure host $\mathrm{pHi}$ in the same host species but with different Symbiodinium types, and the $\mathrm{pH}$ optima of these different symbiont types. There have been few attempts to measure pHi in symbiotic cnidarians (Rands et al., 1993; Venn et al., 2009; Venn et al., 2011), largely because the isolation of viable cnidarian cells is a complex process (Gates and Muscatine, 1992; Domart-Coulon et al., 2001; Helman et al., 2008) and because the imaging of these cells is equally challenging (Fang et al., 1997; Sawyer and Muscatine, 2001). This latter problem particularly arises due to the limited area of host cell cytoplasm available for visualization (Davy et al., 2012) and the chlorophyll autofluorescence of the algal cells that can interfere with signals from 
fluorescent probes. Rands et al. (1993) used an immunocytochemical approach to measure the pHi near the symbiotic dinoflagellates of the sea anemone Anemonia viridis, but while their method suggested an acidic intracellular environment (potentially as low as $\mathrm{pH}$ 5.7), it lacked sufficient resolution. However, the pioneering approach of Venn et al. (2009), which was used in the current study, has provided a platform to do this.

The pHi in $A$. pulchella, when in symbiosis with its homologous symbionts, was slightly lower than previously reported by Venn et al. (2009) for the symbiotic anemone, A. viridis and coral, S. pistillata, both in the light and dark. These authors found the pHi to range between 7.01 and 7.13 in the dark and 7.29 and 7.41 in the light. These values are all within the range reported for other marine invertebrates, with pHi being reported as anywhere between 6.7 for sea urchin eggs (Shen and Steinhard, 1978) to 7.6 for ascidian eggs (Russo et al., 1989). The reasons for the slight differences between these various symbiotic cnidarians is unknown, but it could result from the relative amounts of host-cell cytoplasm available for visualization and the ability to screen the region away from the symbiosome vacuole. That is, the relatively acidic region $(\mathrm{pH}<6.0$; Rands et al., 1993; Venn et al., 2009) immediately surrounding the symbiont cell, which coincides with the symbiosome vacuole, might have been incorporated into measurements here, so moderately lowering the pHi value measured. The increased host-cell pHi in A. pulchella in the light was, however, consistent with the findings of Venn et al. (2009). These authors proposed that this response is related to the dehydration of host cell bicarbonate $\left(\mathrm{HCO}_{3}{ }^{-}\right)$to $\mathrm{CO}_{2}$ for photosynthesis by carbonic anhydrase. This process results in the production of hydroxide $\left(\mathrm{OH}^{-}\right)$ions and, hence, the alkinization of the host cell cytoplasm (Allemand et al., 1998; Furla et al., 1998). While it is apparent that photosynthetic activity influences $\mathrm{pHi}$, no significant differences were noted between the $\mathrm{pHi}$ of the host cells in response to hosting different symbiont types. Of note, however, the influence of Symbiodinium E2 does appear to lead to a trend of a lower $\mathrm{pHi}$ and it is highly likely that greater replication (always a problem given the difficulty of generating aposymbiotic cnidarians) would have led to a significant response. 
The finding that photosynthetic production declined substantially below $\mathrm{pH} 6.75$ for all symbiont types tested (although in the case of the homologous B1 type there was a drop off between $\mathrm{pH} 7.5$ and 7.25 below which the $\mathrm{P}_{\text {gross }}$ was maintained at a low level) is consistent with previously reported trends for free-living algae. Thornton (2009), for example, who investigated photosynthetic production by the marine planktonic diatom Chaetoceros muelleri, found that photosynthesis declined with a corresponding decline in $\mathrm{pH}$ and that, at $\mathrm{pH} 6.8$ and below, the growth rate of the diatom was negatively impacted. He suggested that this decline in photosynthesis during cellular acidosis may be due to less energy being available, which in turn can be linked to two mechanisms: 1) the effect of acidosis on mitochondrial function; and 2) the impact of acidosis on photosynthetic enzyme activity and thylakoid function. It is interesting to note that the algae in this study appear to be living at a $\mathrm{pH}$ below that predicted for seawater as a consequence of ocean acidification ( $\mathrm{pH} 7.4$; Caldeira and Wickett, 2003). However, symbiotic algae may be more adapted to a lower $\mathrm{pH}$. The host cell cytoplasm, as reported in this and previous studies (Rands et al., 1993; Venn et al., 2009, 2011), is more acidic than the external seawater ( $\mathrm{pH} \sim 7.0$ versus $\sim 8.1$ ), and hence symbiosomes typically function under such conditions without any significant impacts on the photosynthetic performance of the symbiotic alga within. However, whether the $\mathrm{pH}$ threshold changes (i.e. becomes less acidic) with time of exposure awaits further investigation. It would be valuable to look at the effect of $\mathrm{pH}$ on the holobiont and compare this between associations with different symbiont types, to identify potential inter and intra-cladal differences.

A range of responses to changes in $\mathrm{pH}$ was reported for the symbiosomes isolated from the different associations. In Chapter 2, the photosynthetic rates of Symbiodinium B1 (both homologous and heterologous) as well as Symbiodinium E2, when in association with $\mathrm{A}$. pulchella, were shown to differ significantly and to range from 0.001 to $0.002 \mathrm{pg} \mathrm{C} \mathrm{cm}^{-3} \mathrm{~h}^{-1}$. A similar trend was noted in this study, in that the rate of $\mathrm{P}_{\text {gross }}$ reached by the different symbiont types at $\mathrm{pH}$ 6.75-7 (i.e. the $\mathrm{pHi}$ measured in the light), differed significantly. The heterologous B1 symbiosomes reached significantly higher rates of $\mathrm{P}_{\text {gross }}$ in this $\mathrm{pH}$ range than the symbiosomes containing the other symbiont types, and the peak value of $0.0008 \mathrm{pg} \mathrm{C} \mathrm{cm}^{3} \mathrm{~h}^{-1}$ was close to that noted in Chapter 2 (i.e. $0.001 \mathrm{pg} \mathrm{C} \mathrm{cm}^{-3} \mathrm{~h}^{-1}$ ). The association with 
heterologous B1 was also the only association for which pHi in the light (i.e. $\mathrm{pH}$ 6.7) fell within the $\mathrm{pH}$ range (i.e. $\mathrm{pH} 6.75$ to 7.5 ) at which the highest photosynthetic performance of the isolated symbiosomes was obtained. This suggests that the pHi enables the symbiont to perform optimally. For heterologous B1, the P:R ratios generally supported the trend observed for $\mathrm{P}_{\text {gross }}$ except that, at $\mathrm{pH} 6.75$, a higher (though non-significant) $\mathrm{R}$ resulted in a lower $\mathrm{P}: \mathrm{R}$ than at $\mathrm{pH}$ 7-7.5. This suggests that the association was nearing a state of being self-supporting with respect to carbon between $\mathrm{pH} 7$ and 7.5, as a P:R ratio of close to 1 was attained (Muscatine et al., 1981).

In the case of the homologous B1 symbionts and Symbiodinium E2, the optimal ambient $\mathrm{pH}$ for photosynthesis did not coincide with the host-cell $\mathrm{pHi}$ in the light. In the case of Symbiodinium E2, this finding is consistent with its low cell-specific rate of photosynthesis when in symbiosis with A. pulchella (Chapter 2), though whether the low photosynthetic rate is caused by a less than optimal $\mathrm{pHi}$ or vice versa is unclear. It is possible that the high respiration rates observed in A. pulchella when in association with Symbiodinium E2 (Chapter 2) may lower the pHi. The improved performance of Symbiodinium E2 at a higher $\mathrm{pH}$ of 8, as evident from the rate of $\mathrm{P}_{\text {gross }}$ and the $\mathrm{P}: \mathrm{R}$, suggests that it would be able to function well in seawater given that the $\mathrm{pH}$ of seawater is approximately 8 (Ball and Stock, 1937). An increasing number of free-living Symbiodinium types are being identified and it has been suggested that the free-living state may be more common than originally suggested (Takabayashi et al., 2012). Therefore, given that type E2 was originally isolated from Wellington Harbour (New Zealand) and that its genotype does not match that of any known New Zealand dinoflagellates (J. Howe, Victoria University of Wellington, pers. comm.), these findings may be indicative of Symbiodinium E2's potential freeliving origin. The disparity between the optimal $\mathrm{pH}$ of the homologous symbionts and the measured pHi is surprising but it could provide a mechanism by which symbiont productivity and hence growth are regulated in hospite. It is known that pre-mitotic controls influence symbiont growth, but what these mechanisms are, is unknown (see review by Davy et al., 2012). Past studies have suggested that the cnidarian host possesses a photosynthesis inhibiting factor that is able to partially inhibit carbon fixation in freshly isolated symbiotic algae (Grant et al., 2006a). This has been 
Chapter 3

proposed to serve as a means of regulating the symbiont density (Grant et al., 2006a). The physiological nature and mechanism of this factor has however, not been established.

In conclusion, this study demonstrates that different Symbiodinium types have different $\mathrm{pH}$ optima with regards to photosynthetic performance and that the $\mathrm{pHi}$ of the host cells may vary depending on the Symbiodinium type present. The study also revealed that the optimal $\mathrm{pHi}$ for photosynthesis does not always match the actual measured $\mathrm{pHi}$. This raises questions about the implications of this mismatch on the functioning of the symbiosis and if it could potentially provide a means of restricting symbiont growth. It should be recognised, however, that exposing isolated symbiosomes to an ambient medium of variable $\mathrm{pH}$, is not a true reflection of the in hospite situation and thus the responses observed in this study may not be the result of $\mathrm{pH}$ change alone. This inability to replicate an in hospite environment is an ongoing problem in the field (Davy et al., 2012). Further work looking at the effect of changes in pHi on the photosynthetic rates of a variety of symbiont types (when in association with A. pulchella) is essential to more accurately assess the potential differences in physiology between them. Also, now that well established methods of measuring pHi in cnidarian cells are available (Venn et al., 2009), it would be interesting to apply them to a wider range of $A$. pulchella-Symbiodinium associations in order to more comprehensively determine the relationship between symbiont diversity and $\mathrm{pHi}$. 


\section{Chapter 4:}

\section{The influence of symbiont type on the glycerol and glucose pools in Aiptasia pulchella}

\subsection{Introduction}

Cnidarian-dinoflagellate symbioses consist of associations between anthozoan cnidarians (i.e. corals, sea anemones, zoanthids, gorgonians) and dinoflagellates (commonly referred to as zooxanthellae) of the genus Symbiodinium (Trench, 1987). A mutualistic relationship exists between the host and symbiont, in that the symbiont receives inorganic nutrients from the host and provides the host with translocated photosynthetic products (Muscatine, 1990). The dinoflagellate symbionts are located within a host-derived vacuole in the gastrodermal cells of the coral host and are separated from the host cytoplasm by a membrane of host origin (Roth et al., 1988) referred to as the symbiosome membrane. This membrane controls the transport of inorganic nutrients to the dinoflagellates (Rands et al., 1993) as well as the translocation of organic products to the host. Glycerol has been suggested as the major extracellular product of Symbiodinium (Muscatine, 1967; Trench, 1971 a, b, c; Grant et al., 1997, 1998; but also see Ishikura et al., 1999; Whitehead and Douglas, 2003; Burriesci et al., 2012), however a range of other mobile compounds have also been identified. These consist of sugars such as glucose and other hexoses (Muscatine et al., 1967; Bil et al., 1991; Ishikura et al., 1999; Whitehead and Douglas, 2003; Burriesci et al., 2012), amino acids such as alanine (Lewis and Smith, 1971; Wang and Douglas, 1999) and glycine (Von Holt, 1968), esters (Von Holt and Von Holt., 1968), alcohols and lipids (Trench, 1971c; Patton et al., 1977; Peng et al., 2011). Given the difficulty of identifying metabolites in hospite, most research to date has focused on the identification of compounds released from isolated dinoflagellates (see review by Davy et al., 2012), which do not provide a true reflection of their behaviour when in symbiosis (Sutton and Hoegh-Guldberg, 1990; Davy et al., 2012). Various approaches identify the translocated metabolites in hospite have been attempted. These include, but are not limited to, studies that track 
the incorporation of ${ }^{14} \mathrm{C}$-labelled compounds into the host tissues (Muscatine and Cernichiari, 1969; Blanquet et al., 1979; Trench, 1971b; Battey and Patton, 1984, 1987), microscopic analyses of cnidarian tissue (Harland et al., 1991, Gautret et al., 1997; Wang and Douglas., 1999; Luo et al., 2009; Chen et al., 2012), and the proteomic and ultrastructural analysis of "lipid bodies" isolated from symbiotic coral cells (Peng et al., 2011). The outcome of this research indicates that the major components of the translocated material are glycerol, amino acids and various lipids. However, there is much debate as to whether glycerol is in fact translocated, with some studies stating that there is no evidence for glycerol release (Rees et al., 1993; Ishikura et al., 1999; Whitehead and Douglas, 2003; Burriesci et al., 2012). More research is therefore necessary to confirm the nature of the compounds translocated from symbiont to host.

Much of the recent research on corals has focused on the interaction between the symbiotic dinoflagellates (genus Symbiodinium) and their coral host during periods of environmental disturbance or change (e.g. coral bleaching induced by elevated seawater temperature). Buddemeier and Fautin (1993) proposed that, following mass bleaching events, changes can occur in the symbiont community structure through recombination with symbionts acquired from the environment following ("switching"). However, although certain corals are capable of acquiring symbionts from the water column, this acquisition is temporary as these infections are not maintained as stable symbioses (Coffroth et al., 2010). There is evidence for postbleaching shifts in the dominant member of a mixed symbiont population (Baker, 2001; Baker et al., 2004; Berkelmans and van Oppen, 2006; Mieog et al., 2007; Jones et al., 2008) and studies have shown that in some coral colonies, the symbiont composition may shift in favour of a Symbiodinium clade or sub-clade ('type') that is physiologically more suited to the new environmental conditions (Stat et al., 2009). For example, Symbiodinium clade D is thought to be more resistant to thermal stress than other clades and has been found to associate with coral colonies after bleaching episodes (Baker, 2001; Rowan, 2004; Abrego et al., 2008). However, this thermal resistance seems to come at a cost to the host, as studies have shown that Symbiodinium clade D may transfer significantly fewer metabolites to its host, leading to a reduction in host growth (Abrego et al., 2008; Cantin et al., 2009). 
Chapter 4

Different clades and sub-clades of symbiotic dinoflagellates have also been found to differentially influence incorporation patterns of translocated carbon in the host's tissues (Loram et al., 2007; Stat et al., 2008; Cantin et al., 2009). For example, Loram et al. (2007) found that when the sea anemone Condylactis gigantea hosted Symbiodinium clade A, a greater proportion of photosynthetically-fixed carbon was incorporated into animal lipids and amino acid pools than when conspecific anemones hosted clade B. These findings suggest that the interactions between specific host-symbiont combinations must play an important role in both the quality and quantity of translocate that is made available to the host. However, we know little about these qualitative and quantitative differences, and how they might influence the capacity of the host to acquire novel symbionts.

The aim of this study was to compare the quality of the photosynthetic translocate made available to the host when infected with different Symbiodinium types. Glucose and glycerol were selected as the metabolites to be tested due to previous studies identifying them as the major metabolites. Amounts of free glycerol and glucose present in the host's tissue both prior to and at different time points after photosynthesis began were analysed, and accumulation rates per symbiont cell calculated in an attempt to compare type-specific changes.

\subsection{Materials and methods}

The sea anemone Aiptasia pulchella was used as the experimental host and infected with different Symbiodinium types (cultured Symbiodinium B1, heterologous Symbiodinium B1 as well as Symbiodinium E2). After 12 weeks, the amounts of free glycerol and glucose present in the tissue of the infected anemones was measured.in the dark, and at different time points after light exposure.

\subsubsection{Experimental organisms}

The sea anemone, Aiptasia pulchella, (originally from the Indo-Pacific region) was cultured at $25{ }^{\circ} \mathrm{C}$ and an irradiance of $100 \mu \mathrm{mol}$ photons $\mathrm{m}^{-2} \mathrm{~s}^{-1}(12 \mathrm{~h}$ light: $12 \mathrm{~h}$ dark $)$ 
Chapter 4

for a period of $>1$ year prior to use. The anemones were fed twice weekly with freshly hatched Artemia sp. over this period. To render the anemones aposymbiotic (i.e. symbiont-free), 120 individuals (oral disc diameter $\sim 10 \mathrm{~mm}$ ) were placed in six $1 \mathrm{~L}$ lightproof containers (20 individuals in each) filled with $0.44 \mu \mathrm{m}$ filtered seawater (FSW) and cold shocked at $4{ }^{\circ} \mathrm{C}$ for 24 hours. The cold-shock process was repeated at weekly intervals for five weeks and the feeding regime was retained between cold-shocks, when care was taken to keep the anemones in low light $(<1$ $\mu \mathrm{mol}$ photons $\left.\mathrm{m}^{-2} \mathrm{~s}^{-1}\right)$. The anemones were then maintained in the dark at $25^{\circ} \mathrm{C}$ for approximately six months until the start of the experiment. The aposymbiotic state of the anemones was checked by exposure to light (12 h light:12 h dark) over a fourweek period, with any change from white to brown colouration being indicative of a residual symbiont population. Fluorescence microscopy (Olympus Provis AX70 microscope using autofluorescence at $100 \times$ magnification) was used to further screen for the presence of symbionts. A subsample of 3 anemones per container was tested. Only anemones that showed no signs of symbiont infection were used for subsequent experiments.

Cultures of Symbiodinium B1 (i.e. not originally isolated from the Aiptasia sp. laboratory culture but from the sea anemone, Aiptasia pallida collected in Long Key, Florida) and E1 (cultured from a single, free-living cell collected from Wellington harbour, New Zealand), as well as a culture of the homologous type (Symbiodinium B1) that was isolated from the laboratory anemone culture, were grown at $25{ }^{\circ} \mathrm{C}$ and an irradiance of $100 \mu \mathrm{mol}$ photons $\mathrm{m}^{-2} \mathrm{~s}^{-1}$ on alight regime of $12 \mathrm{~h}$ light: $12 \mathrm{~h}$ dark. Symbiodinium types were chosen that had previously been shown to have very different photosynthetic performances when in association with $A$. pulchella, i.e. E1 performed poorly whilst both types of Symbiodinium B1 were relatively more successful (see Chapter 2). Furthermore, the heterologous B1 provided a comparison with the homologous B1. The algae were sub-cultured from long-term laboratory stocks and grown in silica-free F/2 medium (Sigma-Aldrich, Auckland, New Zealand) for six weeks prior to use in the infection study.

Symbiodinium types ( $\mathrm{n}=3$ samples per culture) were genetically identified prior to infection (see Appendix 3 for the ITS2 sequences). DNA was extracted from 
Chapter 4

zooxanthellae as described by Logan et al., (2010) and PCR performed using the Symbiodinium-specific ITS2 primers: forward primer (ITSD), 5'-GTG AAT TGC AGA ACT CCG TG-3'; reverse primer (ITS2rev2), 5'-CCT CCG CTT ACT TAT ATG CTT-3'. Reactions were performed in a total volume of $30 \mu \mathrm{L}$ using the MyTaq $^{\mathrm{TM}}$ Mix with an amplification profile consisting of $1 \min 95{ }^{\circ} \mathrm{C}, 35$ cycles of 1 $\min 95{ }^{\circ} \mathrm{C}, 15 \mathrm{~s} 55{ }^{\circ} \mathrm{C}, 10 \mathrm{~s} 72{ }^{\circ} \mathrm{C}$, and a final hold temperature of $4^{\circ} \mathrm{C}$. Single strand conformation polymorphism (SSCP) was performed on all PCR products. PCR products were sequenced by Macrogen Inc. (Seoul, S. Korea). Sequences were aligned using the ClustalW plugin in Geneious Pro 4.8.5 and then edited. A blast search was then performed in the NCBI database in order to identify the sequences (see Table A3.1).

\subsubsection{Infection of aposymbiotic $A$. pulchella with different Symbiodinium types}

Aposymbiotic specimens of $A$. pulchella ( $\mathrm{n}=72$ per treatment) were infected with cultured Symbiodinium cells by pipetting a concentrated drop of the dinoflagellate suspension (approximately 1 million cells $/ \mathrm{mL}$ ) onto the oral disc of each anemone, with a sharp-ended glass pipette. A drop of one-day old Artemia sp. culture was then pipetted onto the oral disc to induce a feeding response and encourage the uptake of the Symbiodinium cells, as described by Davy et al. (1997). Anemones were kept in FSW in $20 \mathrm{~mL}$ glass containers that received a water change 8 hours after infection. The infected anemones were then left for 12 weeks to form a stable association with their respective symbiont (see Chapter 2). The feeding regime during the 12-week period was maintained as per pre-infection. After 12 weeks, zooxanthellae were isolated from three anemones per treatment and their genetic identity confirmed, using the isolation and molecular techniques described above. All treatments were found to harbour the same Symbiodinium type as the one introduced at the start of the experiment (see Appendix 3 for the ITS2 sequences).

\subsubsection{Levels of glycerol and glucose in host tissues}

All the infected anemones were kept in darkness for 24 hours at a temperature of $25{ }^{\circ} \mathrm{C}$. The anemones were last fed 1 week prior to the dark treatment. After the dark 
Chapter 4

adaptation period 36 anemones per treatment were exposed to light at $400 \mu \mathrm{mol}$ photons $\mathrm{m}^{-2} \mathrm{~s}^{-1}$ (light-saturation for photosynthesis) and 6 anemones per treatment were used for the dark treatment (i.e. $\mathrm{t}=0$ ). Six anemones from each treatment were homogenized individually in $1 \mathrm{~mL}$ of FSW using a tissue grinder at each time point (i.e. $0,1,3,6,9,12$ and 24 hours after light exposure). A preliminary study was completed in order to demonstrate that 24 hours of continuous light did not negatively impact the photosynthetic rate of the symbionts. The homogenate was centrifuged at $\times 4500 \mathrm{~g}$ for $3 \mathrm{~min}$. The supernatant was stored at $-20{ }^{\circ} \mathrm{C}$ for quantification of glycerol and glucose as described below, as well as protein. Protein was quantified by measuring absorbance of the supernatant at $280 \mathrm{~nm}$ using a ND 1000 spectrophotometer (Nanodrop, Thermo Fisher Scientific Inc.); FSW was used for blank measurements. The pellet was resuspended in $1 \mathrm{~mL}$ of FSW and centrifuged again at $\times 1000 \mathrm{~g}$ for $5 \mathrm{~min}$. This was repeated three times. A haemocytometer and light microscope $(100 \times$ magnification $)$ were used to estimate the total number of algal cells per $\mathrm{mL}$ of anemone homogenate $(n=6$ counts per anemone).

In a second, modified version of this experiment, anemones were treated with the respiratory inhibitor sodium cyanide $(\mathrm{NaCN})$ in an attempt to minimise the influence of host metabolic activity on the accumulation of translocated carbon compounds. The experimental protocol was identical to that described above except that: (i) after the dark adaptation period, 18 anemones per treatment were placed in individual glass vials containing a $2.5 \times 10^{-5} \mathrm{M} \mathrm{NaCN}$ solution (in FSW and adjusted to $\mathrm{pH}$ 8.2); and (2) 6 anemones per treatment were processed at 0,30 and 60 min after light exposure.

\subsubsection{Carbon fluxes in isolated Symbiodinium}

To prepare a host tissue homogenate, five anemones of the same nutritional history were homogenized together in $3 \mathrm{~mL}$ of FSW using a tissue grinder. The homogenate was then centrifuged at $\times 1000 \mathrm{~g}$ for $3 \mathrm{~min}$, and the supernatant retained and stored at $-20{ }^{\circ} \mathrm{C}$. This homogenate was used for all subsequent incubations. 
Chapter 4

To obtain isolated Symbiodinium cells, 12 re-infected anemones per treatment were individually homogenized in $1 \mathrm{~mL}$ FSW using a tissue grinder. The homogenate was centrifuged at $\times 4500 \mathrm{~g}$ for $3 \mathrm{~min}$. The pellet was resuspended in $1 \mathrm{~mL} F S W$ and centrifuged again at $\times 1000 \mathrm{~g}$ for $5 \mathrm{~min}$. This washing process was repeated three times. The algal pellet was then diluted to $10^{6}$ cells $\mathrm{mL}^{-1}$ in FSW. An aliquot $(300 \mu \mathrm{L})$ of the algal suspension from each anemone was then placed in a $1.5-\mathrm{mL}$ microcentrifuge tube together with $300 \mu \mathrm{L}$ of the previously prepared host homogenate. The tubes were placed in darkness for 24 hours at a temperature of $25{ }^{\circ} \mathrm{C}$, after which they were exposed to light for an hour $\left(400 \mu \mathrm{mol}\right.$ photons $\mathrm{m}^{-2} \mathrm{~s}^{-1}$; light-saturation for photosynthesis). At 0,30 and $60 \mathrm{~min}$ post light exposure, 4 microcentrifuge tubes per treatment were individually vortexed and centrifuged at $\times 1000 \mathrm{~g}$ for $3 \mathrm{~min}$ in order to separate the algal and animal fractions. The supernatant was stored at $-20{ }^{\circ} \mathrm{C}$ for glycerol and glucose quantification as described below. The pellet was resuspended in $1 \mathrm{~mL}$ FSW and algal cell counts were performed as described earlier.

\subsubsection{Glucose and glycerol assays}

All samples were analysed using the Abcam Free Glucose and Glycerol Assay kits (Sapphire Bioscience, NZ, Ltd.) in order to measure the free glycerol and glucose concentrations. Samples and standards were prepared according to the protocol provided. The assays involve the enzymatic oxidation of glycerol/glucose to generate a product which reacts with a dye to generate colour $(\lambda=570 \mathrm{~nm})$ in the case of glucose and fluorescence $(\mathrm{Ex} / \mathrm{Em}=535 / 587 \mathrm{~nm})$ in the case of glycerol. A microtiter plate reader (EnSpire 2300 Multilabel Reader; PerkinElmer; Waltham, MA, USA) was used for these measurements.

In order to calculate the amount of glycerol or glucose present in each sample, the value derived from the blank glucose and glycerol standards was subtracted from all the sample readings to correct for background fluorescence. The standard curve was plotted as fluorescence vs. concentration of glycerol/glucose (nmol). The sample fluorescence/absorbance readings were applied to the standard curve in order to calculate the concentration of glycerol/glucose in each sample. For the intact 
Chapter 4

symbiosis experiments (both in the presence and absence of $\mathrm{NaCN}$ ) the sample concentrations were normalised to the total host protein present in each sample (i.e $\mathrm{nmol} \mathrm{mg}{ }^{-1}$ protein or $\mathrm{pmol} \mathrm{mg}^{-1}$ protein) in order to track the change in concentration in the host tissue. The values were also normalised to the algal cell density present in the association from which the sample was obtained (i.e. nmol/ $10^{6}$ algal cells) so to to directly compare the contribution of the different symbiont types to the host tissue metabolite pools. The glycerol/glucose concentration at $\mathrm{t}=0$ was than subtracted from each subsequent time point in order to determine the increase in the metabolite concentration from the baseline level. Glycerol and glucose concentrations released by isolated Symbiodinium were normalised to algal cell density only (i.e. nmol/10 ${ }^{6}$ algal cells) and the change from the baseline level was calculated as described above.

\subsubsection{Statistical analyses}

Data were tested for normality using the Kolmogorov-Smirnoff test and for homogeneity of variance using the Bartlett test. Comparisons of glycerol and glucose host tissue concentration (normalised to host protein and to algal cell density) between the different symbiotic associations and time points (in the presence and absence of $\mathrm{NaCN}$ ) were made using one-way analysis of variance (ANOVA, $\alpha=$ 0.05), followed by LSD post hoc tests for normal data using PASW (Predictive Analytics SoftWare, Version 18). Analyses of the glycerol and glucose concentrations released by isolated Symbiodinium were performed in the same manner.

\subsection{Results}

\subsubsection{Glycerol and glucose levels in the host tissues}

The average algal densities measured across all time points differed between the associations tested (one-way ANOVA, d.f. $=2 ; 15, \mathrm{~F}=508.7, \mathrm{p}<0.005$ for all treatments). The association with Symbiodinium E2 had a significantly higher algal density $\left(4.00 \times 10^{6}\right.$ cells $\mathrm{mg}^{-1}$ protein $)$ than the associations with the other two types 
$\left(1.25 \times 10^{6}\right.$ cells $\mathrm{mg}^{-1}$ protein and $0.52 \times 10^{6}$ cells $\mathrm{mg}^{-1}$ protein for the associations with heterologous Symbiodinium B1 and homologous Symbiodinium B1, respectively; one-way ANOVA, LSD post-hoc test, $\mathrm{p}<0.005$ for both treatments). Also, the association with heterologous Symbiodinium B1 had a significantly higher algal density than the association with homologous Symbiodinium B1 (one-way ANOVA, LSD post-hoc test, $\mathrm{p}<0.005$ for both treatments).

There was a significant increase in the host tissue glycerol concentration (nmol mg ${ }^{-1}$ protein) over time when the anemones with homologous and heterologous Symbiodinium B1 were incubated in the light (Figure 4.1A; one-way ANOVA, d.f. = $6 ; 15-21, \mathrm{~F}=2.5-5.6, \mathrm{p}<0.05$ for all treatments). The concentration increased from to 0.014 to $0.039 \mathrm{nmol} \mathrm{mg}{ }^{-1}$ protein and 0.024 to $0.068 \mathrm{nmol} \mathrm{mg}$ protein for associations with homologous B1 and the heterologous B1 respectively. The association with Symbiodinium E2 displayed no change in host tissue glycerol concentration over time (one-way ANOVA, d.f. $=6 ; 24, \mathrm{~F}=0.6, \mathrm{p}>0.05$ ). Differences in glycerol concentration between the associations were noted at 0 and 1 hour (one-way ANOVA, d.f. $=2 ; 8-9, \mathrm{~F}=9.2-41.0, \mathrm{p}<0.05$ ) with the heterologous Symbiodinium B1 and Symbiodinium E2 associations displaying significantly higher levels of glycerol $\left(0.02\right.$ and $0.04 \mathrm{nmol} \mathrm{mg}^{-1}$ protein at 0 hours and 0.01 and 0.03 $\mathrm{nmol} \mathrm{mg}{ }^{-1}$ protein at 1 hour for associations with heterologous Symbiodinium B1 and Symbiodinium E2, respectively) than the association with homologous Symbiodinium B1 (0.01 and $0.009 \mathrm{nmol} \mathrm{mg}^{-1}$ protein for 0 and 1 hour, respectively; one-way ANOVA, LSD post-hoc test, $\mathrm{p}<0.05$ for both treatments). The concentration in the different associations did not differ after 24 hours in the light (one-way ANOVA, d.f. $=2 ; 5, \mathrm{~F}=1.5, \mathrm{p}>0.05$ ).

The trends in host tissue glycerol concentration seen when normalising to cell density were not the same as those observed when normalising to host protein as the algal cell densities present in the different associations varied. The cell-specific glycerol concentration (nmol/10 $0^{6}$ algal cells, measured as change from the baseline concentration at 0 hours) changed with time in all cases (one-way ANOVA, d.f. $=5$; $12-16, \mathrm{~F}=3.5-24.5, \mathrm{p}<0.05$ for all comparisons), but the patterns of accumulation were again different between the different Symbiodinium types (Figure 4.1B). In the 
Chapter 4

case of homologous Symbiodinium B1, the cell-specific glycerol concentration was significantly higher at 1, 3, 6 and 9 hours, where it ranged from 25.10 to 40.53 $\mathrm{nmol} / 10^{6}$ algal cells, than at 12 and 24 hours $\left(10.80\right.$ and $6.51 \mathrm{nmol} / 10^{6}$ algal cells, respectively; one-way ANOVA with LSD post-hoc test, $\mathrm{p}<0.05$ for all comparisons). With heterologous Symbiodinium B1 type, on the other hand, the cellspecific glycerol concentration was significantly higher at 12 hours $\left(20.65 \mathrm{nmol} / 10^{6}\right.$ algal cells) than at all the other time points where it ranged from 3.90 to 9.50 $\mathrm{nmol} / 10^{6}$ algal cells (one-way ANOVA with LSD post-hoc test, $\mathrm{p}<0.005$ for all comparisons); it was also higher at 24 hours $\left(9.50 \mathrm{nmol} / 10^{6}\right.$ algal cells $)$ than at 1,3 and 6 hours where it ranged from 3.90 to $5.15 \mathrm{nmol} / 10^{6}$ algal cells (one-way ANOVA with LSD post-hoc test, $\mathrm{p}<0.05$ for all comparisons). The association with Symbiodinium E2 had the highest cell-specific glycerol concentration at 9 hours when compared to the other time points $\left(11.42 \mathrm{nmol} / 10^{6}\right.$ algal cells; one-way ANOVA with LSD post-hoc test, $p<0.005$ ), while the concentrations at 6 and 12 hours (2.90 and $5.27 \mathrm{nmol} / 10^{6}$ algal cells, respectively) were also higher than for the remaining time points (except 9 hours) where it ranged from 0.48 to $1.22 \mathrm{nmol} / 10^{6}$ algal cells (one-way ANOVA with LSD post-hoc test, $\mathrm{p}<0.05$ for all comparisons). These different accumulation patterns led to differences between the various Symbiodinium types at some of the time points (one-way ANOVA, d.f. $=2 ; 4-9, \mathrm{~F}=$ 8.0-16.7, $\mathrm{p}<0.05$ for all comparisons). In particular, the symbiosis with the homologous Symbiodinium B1 type contained more glycerol per symbiont cell (25.09-40.53 $\mathrm{nmol} / 10^{6}$ algal cells versus 0.48 to $5.15 \mathrm{nmol} / 10^{6}$ algal cells) at $1,3,6$ and 9 hours than the symbioses with the other two types (one-way ANOVA with LSD post-hoc test, $\mathrm{p}<0.05$ for all comparisons). At 12 hours, on the other hand, the glycerol concentration in the symbiosis with the heterologous Symbiodinium B1 was significantly higher than for the symbioses with the other two symbiont types $(20.65$ $\mathrm{nmol} / 10^{6}$ algal cells versus 10.80 and $5.27 \mathrm{nmol} / 10^{6}$ algal cells for homologous Symbiodinium B1 type and Symbiodinium E2, respectively) (one-way ANOVA with LSD post-hoc test, $\mathrm{p}<0.05$ for all comparisons). At 24 hours no significant differences between the symbioses were displayed (one-way ANOVA, d.f. $=2 ; 8, \mathrm{~F}$ $=4.2, \mathrm{p}<0.05$ for all comparisons). 

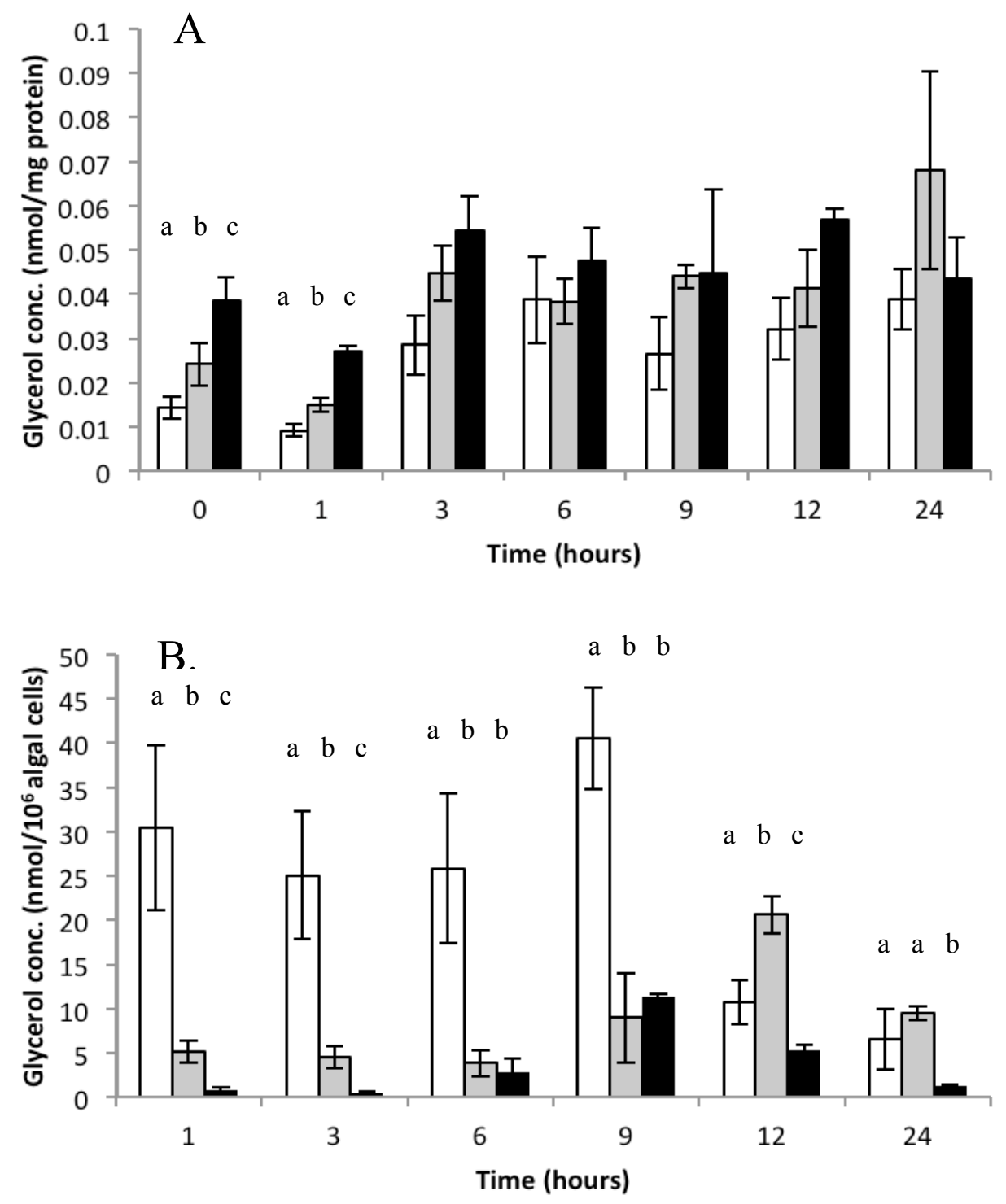

Figure 4.1. Concentration of glycerol in tissues of $A$. pulchella when infected with different Symbiodinium types versus time in the light. (A) Standardised to host protein content. (B) Standardised to algal cell number, after subtracting the baseline glycerol concentration at $0 \mathrm{~h}$. White bars, homologous Symbiodinium B1; grey bars, heterologous Symbiodinium B1; black bars, Symbiodinium E2. At each time point, associations assigned different letters are significantly different from each other $(\mathrm{p}<0.05)$. Values are means $\pm S E ; n=5$ or 6 per time point. The baseline glycerol concentration was $27.81 \pm 5.65,6.96 \pm 1.05$ and $2.86 \pm 0.12$ $\mathrm{nmol} / 10^{6}$ algal cells for the homologous Symbiodinium B1, heterologous Symbiodinium B1 and Symbiodinium E2 respectively.

All three associations displayed a significant increase in the concentration of glucose in the host tissues over time in the light (Figure 4.2A; one-way ANOVA, d.f. $=6$; 8- 
$24, \mathrm{~F}=5.5-12.5, \mathrm{p}<0.005$ for all treatments) increasing from 0.05 to $0.6 \mathrm{pmol} \mathrm{mg}^{-1}$ protein for the association with the homologous SymbiodiniumB1, 0.08 to 0.9 pmol $\mathrm{mg}^{-1}$ protein for the association with the heterologous Symbiodinium B1, and 0.17 to $0.63 \mathrm{pmol} \mathrm{mg}^{-1}$ protein for the association with Symbiodinium E2 (Figure 4.2A). For the association with the homologous B1, at 12 and 24 hours, the amount of glucose present ( 0.80 and $0.60 \mathrm{pmol} \mathrm{mg}^{-1}$ protein, respectively) was significantly higher than at the preceding time points where it ranged from 0.01 to $0.11 \mathrm{pmol} \mathrm{mg}^{-1}$ protein (one-way ANOVA, LSD post-hoc test, $\mathrm{p}<0.05$ for all comparisons). The association with the heterologous B1 had significantly higher concentrations of glucose at 9, 12 and 24 hours $\left(0.72,0.68\right.$ and $0.89 \mathrm{pmol} \mathrm{mg}^{-1}$ protein, respectively) than at the earlier time points where it ranged from 0.03 to $0.18 \mathrm{pmol} \mathrm{mg}^{-1}$ protein (one-way ANOVA, LSD post-hoc test, $\mathrm{p}<0.005$ for all comparisons). The amount of glucose present in the host tissues of the Symbiodinium E2 symbiosis after 24 hours of light exposure $\left(0.63 \mathrm{pmol} \mathrm{mg}^{-1}\right.$ protein) was significantly higher than at all of the preceding time points where it ranged from 0.05 to $0.38 \mathrm{pmol} \mathrm{mg}^{-1}$ protein (one-way ANOVA, LSD post-hoc test, $\mathrm{p}<0.05$ for all comparisons $)$, and at 12 hours $\left(0.35 \mathrm{pmol} \mathrm{mg}^{-1}\right.$ protein) it was significantly higher than at 0,1 and 6 hours $(0.17,0.05$ and 0.20 respectively; one-way ANOVA, LSD post-hoc test, $\mathrm{p}<0.05$ for both comparisons). This increased accumulation of glucose with time in all three symbioses meant that there were few significant differences between them. Indeed, the only significant difference was at 9 hours, when the symbiosis with the heterologous Symbiodinium B1 had a greater concentration of glucose in its host tissues than did the other two associations $(0.72$ pmol $\mathrm{mg}^{-1}$ protein $v$ s. 0.1 and $0.14 \mathrm{pmol} \mathrm{mg}^{-1}$ protein for the associations with homologous B1 and E2, respectively) (one-way ANOVA, d.f. $=2 ; 9, \mathrm{~F}=32.1, \mathrm{p}<$ 0.005 for both comparisons).

The trends in host tissue glucose concentration (as noted previously with glycerol) seen when normalising to cell density were not the same as that observed when normalising to host protein due to variable algal cell densities present in the different associations. When normalized to symbiont density ( $\mathrm{nmol} / 10^{6}$ algal cells), there was a tendency towards an accumulation of glucose as time progressed for all the symbioses (Figure 4.2B; one-way ANOVA, d.f. $=5 ; 12-14, \mathrm{~F}=3.0-11.2, \mathrm{p}<0.005$ ). With homologous Symbiodinium B1, the cell-specific glucose concentration was 
significantly greater at 12 hours $\left(1.63 \mathrm{nmol} / 10^{6}\right.$ algal cells $)$ than at any of the other time points where it ranged from -0.03 to $0.45 \mathrm{nmol} / 10^{6}$ algal cells (one-way ANOVA with LSD post-hoc test, $\mathrm{p}<0.005$ for all comparisons), and the cellspecific glucose concentration in the association with heterologous Symbiodinium B1 was significantly higher at 9,12 and 24 hours $\left(0.49,0.27\right.$ and $0.32 \mathrm{nmol} / 10^{6}$ algal cells respectively) than at the previous time points where it ranged from 0.003 to $0.05 \mathrm{nmol} / 10^{6}$ algal cells (one-way ANOVA with LSD post-hoc test, $\mathrm{p}<0.05$ for all comparisons). Similarly, with Symbiodinium E2 the cell-specific glucose concentration was significantly greater at 24 hours $\left(0.06 \mathrm{nmol} / 10^{6}\right.$ algal cells $)$ than at the earlier time points where it ranged from 0.003 to $0.02 \mathrm{nmol} / 10^{6}$ algal cells (oneway ANOVA with LSD post-hoc test, $\mathrm{p}<0.005$ for all comparisons). Despite these similar trends, however, the rates of glucose accumulation differed between the different symbioses, with there being significant differences at 1, 9 and 12 hours (one-way ANOVA, d.f. $=2 ; 4-8, \mathrm{~F}=5.1-8.1, \mathrm{p}<0.05$ for all comparisons). At 1 hour, the cell-specific concentration of glucose was significantly greater in the presence of the heterologous Symbiodinium B1 than with the other two symbionts $\left(0.04 \mathrm{nmol} / 10^{6}\right.$ algal cells $v s .-0.03$ and $0.01 \mathrm{nmol} / 10^{6}$ algal cells for the association with homologous Symbiodinium B1 and Symbiodinium E2, respectively; one-way ANOVA with LSD post-hoc test, $\mathrm{p}<0.05$ for both comparisons). At 9 and 12 hours, the associations with both the homologous and heterologous Symbiodinium B1 showed significantly higher concentrations of glucose per symbiont cell $(0.26$ and $0.49 \mathrm{nmol} / 10^{6}$ algal cells at 9 hours, and 1.63 and $0.27 \mathrm{nmol} / 10^{6}$ algal cells at 12 hours, respectively) than did the association with Symbiodinium E2 (0.02 and 0.06 nmol/10 6 algal cells at 9 and 12 hours, respectively) one-way ANOVA with LSD post-hoc test, $\mathrm{p}<0.05$ for all comparisons). Of note, the average cell-specific glucose concentration was about 7-fold higher in the presence of the homologous Symbiodinium B1 at 12 hours than for the heterologous B1. 
Chapter 4
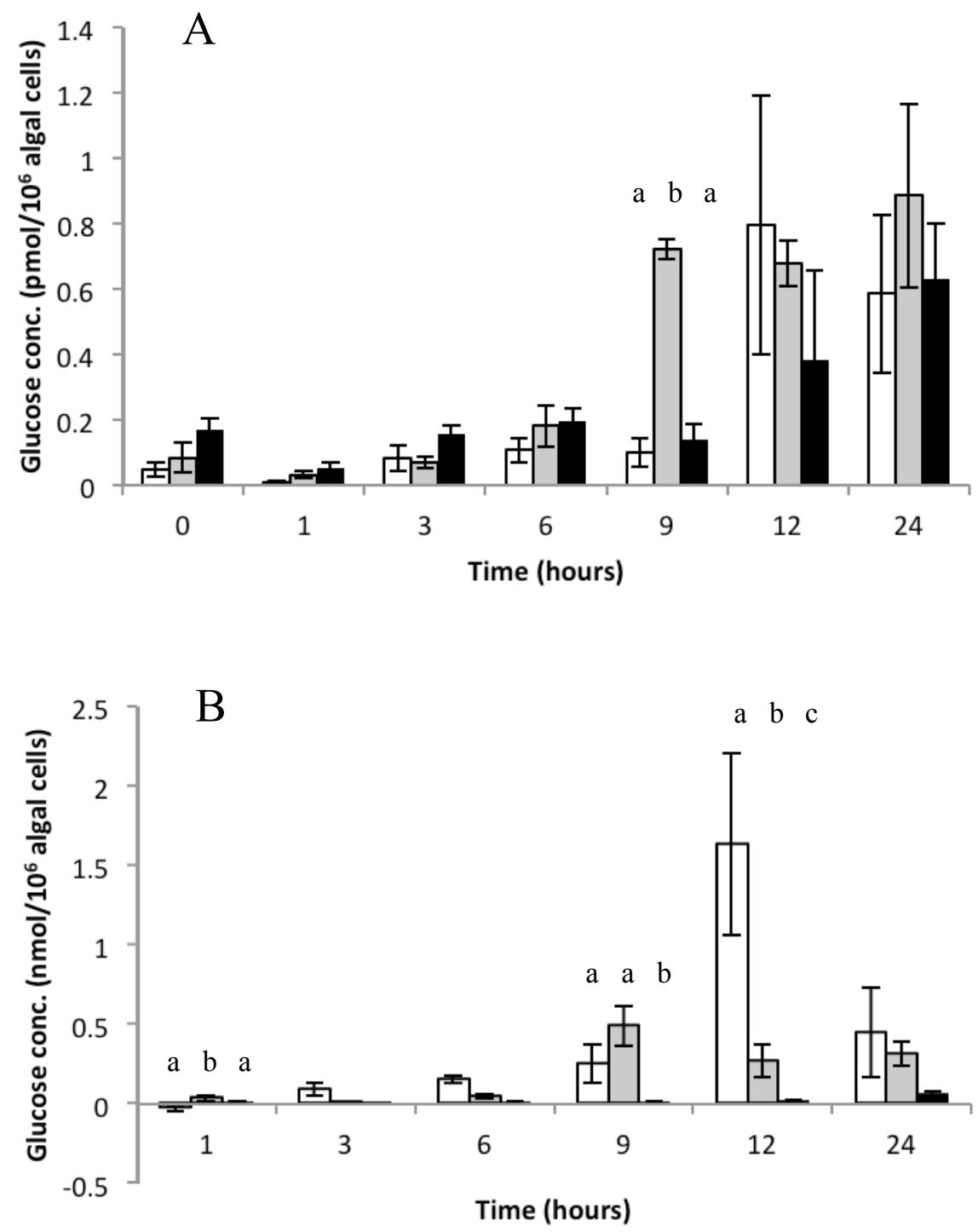

Figure 4.2. Concentration of glucose in tissues of A. pulchella when infected with different Symbiodinium types versus time in the light. (A) Standardised to host protein content. (B) Standardised to algal cell number, after subtracting the baseline glucose concentration at $0 \mathrm{~h}$. White bars, homologous Symbiodinium B1; grey bars, heterologous Symbiodinium B1; black bars, Symbiodinium E2. At each time point, associations assigned different letters are significantly different from each other $(\mathrm{p}<0.05)$. Values are means $\pm \mathrm{SE} ; \mathrm{n}=5$ or 6 per time point. The baseline glucose concentration was $0.10 \pm 0.04,0.02 \pm 0.003$ and $0.006 \pm 0.0008$ $\mathrm{nmol} / 10^{6}$ algal cells for the homologous Symbiodinium B1, heterologous Symbiodinium B1 and Symbiodinium E2 respectively. 
Chapter 4

\subsubsection{Glycerol and glucose levels in the host tissue in the presence of $\mathrm{NaCN}$}

When incubated with $\mathrm{NaCN}$, the different associations displayed similar trends to each other with respect to the accumulation of glycerol (Figure 4.3A). In all the associations, the concentration of glycerol (nmol/mg protein) in the host tissues increased over the first $30 \mathrm{~min}$ of the the incubation (ranging from 0.006 to $0.012 \mathrm{nmol} \mathrm{mg}^{-1}$ at $0 \mathrm{~min}$ and 0.023 to $0.030 \mathrm{nmol} \mathrm{mg}^{-1}$ protein at $30 \mathrm{~min}$ ), though in the case of the association with the homologous Symbiodinium B1 this increase only approached significance (one-way ANOVA, d.f. $=2 ; 6-9, \mathrm{~F}=6.2-19.1, \mathrm{p}<0.05$, except for homologous B1 where $\mathrm{p}=0.06$ ). Glycerol concentration then declined between 30 and $60 \mathrm{~min}$ in all cases (ranging from 0.023 to $0.030 \mathrm{nmol} \mathrm{mg}^{-1}$ protein at $30 \mathrm{~min}$ at $30 \mathrm{~min}$ and 0.011 to $0.020 \mathrm{nmol} \mathrm{mg} \mathrm{m}^{-1}$ protein at $60 \mathrm{~min}$; one-way ANOVA with LSD post-hoc test, $\mathrm{p}<0.05$ for all comparisons). No significant differences, however, were detected between the different associations at the various time points (one-way ANOVA, d.f. $=2 ; 7-10, \mathrm{~F}=0.1-4.3, \mathrm{p}>0.05$ for all comparisons).

In the case of the cell-specific glycerol concentration (nmol/10 ${ }^{6}$ algal cells, measured as change from the baseline concentration at $0 \mathrm{~min}$ ), only the association with homologous Symbiodinium B1 changed significantly between 30 and 60 min of incubation, when it dropped from 58.2 to $0.4 \mathrm{nmol} / 10^{6}$ algal cells (Figure 4.3B; oneway ANOVA, d.f. $=1 ; 5, F=11.7, p<0.05)$. Significant differences between the types were seen at both 30 and $60 \mathrm{~min}$ (one-way ANOVA, d.f. $=2 ; 7-9, \mathrm{~F}=8.8-20.1$, $\mathrm{p}<0.005$ for all comparisons). At $30 \mathrm{~min}$, the associations with homologous and heterologous Symbiodinium B1 displayed a greater increase in host tissue glycerol concentration per cell $\left(58.21 \mathrm{nmol} / 10^{6}\right.$ algal cells and $15.05 \mathrm{nmol} / 10^{6}$ algal cells, respectively) than did the Symbiodinium E2 association $\left(2.31 \mathrm{nmol} / 10^{6}\right.$ algal cells), with homologous B1 symbiosis exhibiting the greatest increase (one-way ANOVA with LSD post-hoc test, $\mathrm{p}<0.05$ for all comparisons). At $60 \mathrm{~min}$, the association with the heterologous Symbiodinium B1 showed a significantly greater increase in glycerol concentration per cell $\left(3.71 \mathrm{nmol} / 10^{6}\right.$ algal cells $)$ than the other two associations $\left(0.39\right.$ and $-0.32 \mathrm{nmol} / 10^{6}$ algal cells for the association with homologous 
Chapter 4

Symbiodinium B1 and Symbiodinium E2, respectively; one-way ANOVA with LSD post-hoc test, $\mathrm{p}<0.005$ for all comparisons).
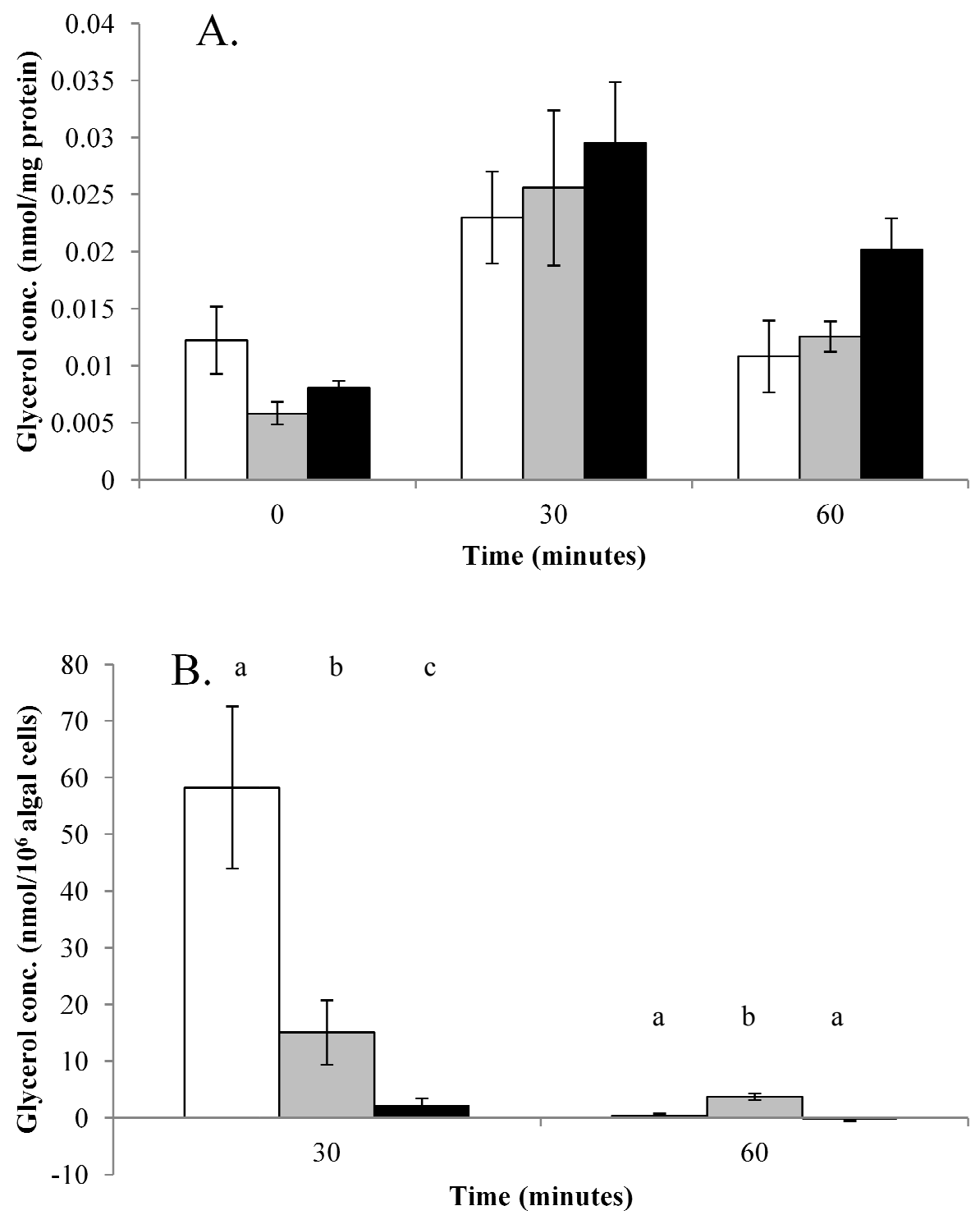

Figure 4.3. Concentration of glycerol in A. pulchella tissues when incubated for an hour in the light in the presence of the respiratory inhibitor $\mathrm{NaCN}$. (A) Standardised to host protein content. (B) Standardised to algal cell number, after subtracting the baseline glycerol concentration at $0 \mathrm{~min}$. White bars, homologous Symbiodinium B1; grey bars, heterologous Symbiodinium B1; black bars, Symbiodinium E2. At each time point, associations assigned different letters are significantly different from each other $(p<0.05)$. Values are means \pm SE; $\mathrm{n}=5$ or 6 per time point. 
Chapter 4

In the case of glucose in the host tissues, all three associations demonstrated an increase in concentration per unit protein between 0 and $30 \mathrm{~min}$, from $0.038-0.051 \mathrm{pmol} / \mathrm{mg}$ protein to $0.124-0.132 \mathrm{pmol} / \mathrm{mg}$ protein (Figure 4.4A; one-way ANOVA with LSD post-hoc test, d.f. $=2 ; 7-9, \mathrm{~F}=7.1-24.8, \mathrm{p}<0.05$ for all comparisons). Only the association with heterologous Symbiodinium B1, however, showed a further increase between 30 and $60 \mathrm{~min}$, reaching a peak of $0.187 \mathrm{pmol} / \mathrm{mg}$ protein (one-way ANOVA with LSD post-hoc test, $\mathrm{p}<0.05$ ). This trend meant that at $60 \mathrm{~min}$ the association with heterologous B1 had a significantly higher concentration of glucose than the other two associations (one-way ANOVA with LSD post-hoc test, d.f. $=2 ; 8, \mathrm{~F}=5.1, \mathrm{p}<0.05$ ); no other significant differences were apparent between the three symbiont types (one-way ANOVA, d.f. $=2 ; 8-9, \mathrm{~F}$ $=0.1-0.7, \mathrm{p}<0.05)$.

With respect to the cell-specific glucose concentration (nmol/10 ${ }^{6}$ algal cells), only the association with the homologous Symbiodinium B1 changed between 30 and $60 \mathrm{~min}$, with a drop from 0.20 to $0.16 \mathrm{nmol} / 10^{6}$ algal cells (Figure 4.4B; one-way ANOVA with LSD post-hoc test, d.f. $=1 ; 5, \mathrm{~F}=83.8, \mathrm{p}<0.005)$. However, significant differences were apparent between the associations at both 30 and $60 \mathrm{~min}$ (one-way ANOVA, d.f. $=2 ; 8-9, \mathrm{~F}=19.9-3274.5, \mathrm{p}<0.005$ for all treatments), with the homologous and heterologous Symbiodinium B1 associations having significantly greater cell-specific glucose concentrations $\left(0.20\right.$ and $0.07 \mathrm{nmol} / 10^{6}$ algal cells at $30 \mathrm{~min}$, and 0.16 and $0.11 \mathrm{nmol} / 10^{6}$ algal cells at $60 \mathrm{~min}$ for the associations with homologous and heterologous B1, respectively) than the Symbiodinium E2 association at both time points $\left(0.02\right.$ and $0.02 \mathrm{nmol} / 10^{6}$ algal cells for 30 and $60 \mathrm{~min}$, respectively; one-way ANOVA with LSD post-hoc test, $\mathrm{p}<0.005$ for all comparisons). Furthermore, the homologous B1 association had a greater cellspecific concentration of glucose than the heterologous B1 association at both time points (one-way ANOVA with LSD post-hoc test, $\mathrm{p}<0.05$ for both comparisons). 
Chapter 4
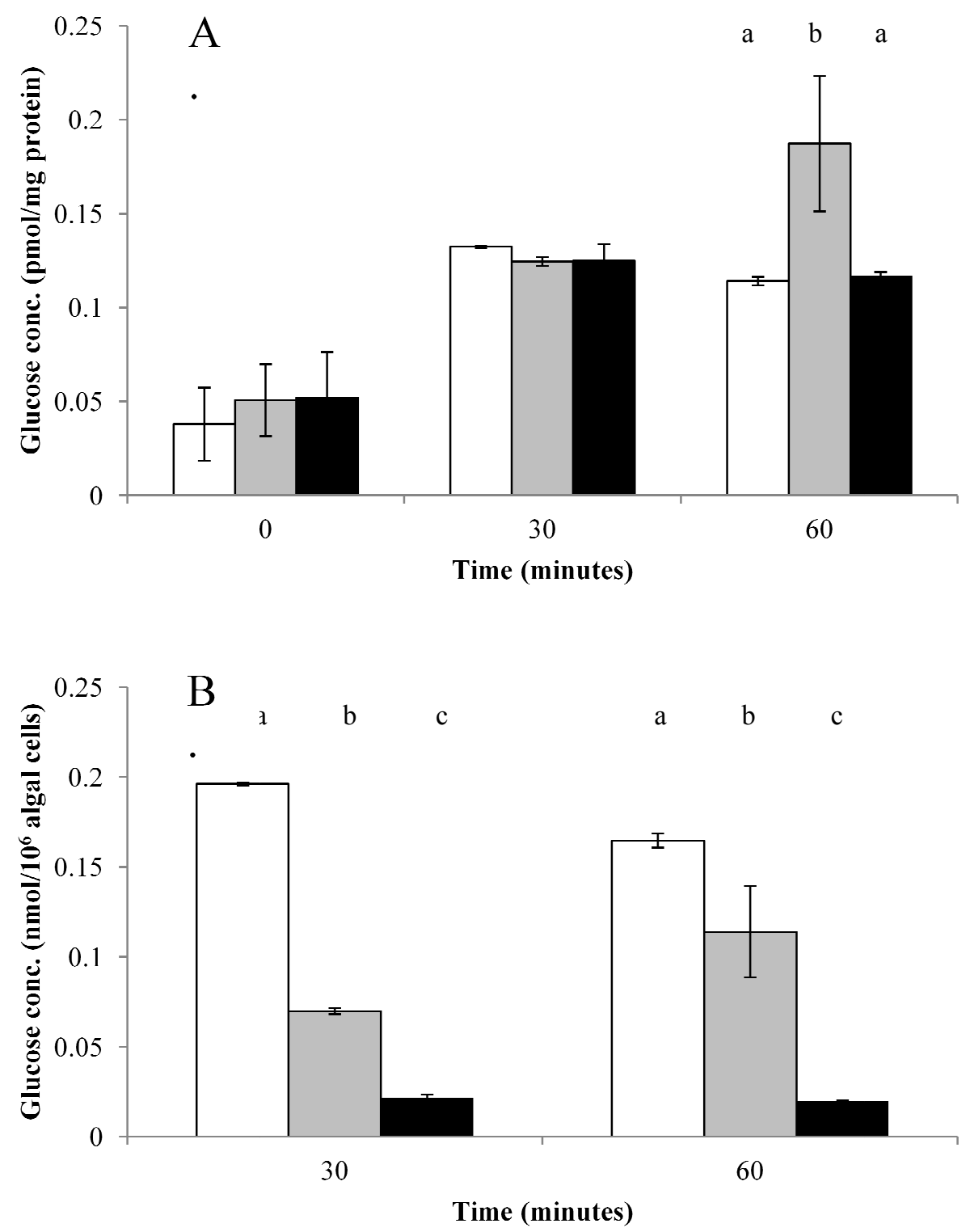

Figure 4.4. Concentration of glucose in A. pulchella tissues when incubated for an hour in the light in the presence of the respiratory inhibitor $\mathrm{NaCN}$. (A) Standardised to host protein content. (B) Standardised to algal cell number, after subtracting the baseline glucose concentration at $0 \mathrm{~min}$. White bars, homologous Symbiodinium B1; grey bars, heterologous Symbiodinium B1; black bars, Symbiodinium E2. At each time point, associations assigned different letters are significantly different from each other $(p<0.05)$. Values are means \pm SE; $\mathrm{n}=5$ or 6 per time point. 
Chapter 4

\subsubsection{Carbon fluxes in isolated Symbiodinium}

When a host homogenate was used to stimulate metabolite release by the different Symbiodinium types, they all exhibited similar trends for both glycerol and glucose (Figure 4.5). Glycerol concentration increased from $14.4 \mathrm{nmol} / 10^{6}$ algal cells at $0 \mathrm{~min}$ to $104.4,64.5$ and $72.0 \mathrm{nmol} / 10^{6}$ algal cells at $30 \mathrm{~min}$ for the homologous Symbiodinium B1, heterologous Symbiodinium B1 and Symbiodinium E2, respectively (one-way ANOVA with LSD post-hoc test, d.f. $=2 ; 6, \mathrm{~F}=4.9-8.5, \mathrm{p}<$ 0.05 for all comparisons) and then remained unchanged between 30 and 60 min (oneway ANOVA with LSD post-hoc test, $\mathrm{p}>0.05$ ). Glucose concentration followed a similar pattern, with an increase from $0.29 \mathrm{nmol} / 10^{6}$ algal cells at 0 min to $0.54,0.41$, $0.40 \mathrm{nmol} / 10^{6}$ algal cells for the homologous Symbiodinium B1, heterologous Symbiodinium B1 and Symbiodinium E2, respectively (one-way ANOVA with LSD post-hoc test, d.f. $=2 ; 6, \mathrm{~F}=10.4-31.8, \mathrm{p}<0.05$ for all comparisons) and no change between 30 and 60 min (one-way ANOVA with LSD post-hoc test, $\mathrm{p}>0.05$ ).

There were differences in the amount of glycerol and glucose released by the different Symbiodinium types at 30 and $60 \mathrm{~min}$ (one-way ANOVA, d.f. $=2 ; 6, \mathrm{~F}=$ 5.3-51.1, p $<0.05$ for all comparisons). By $30 \mathrm{~min}$, the homologous Symbiodinium B1 had released significantly more glycerol and glucose than had Symbiodinium E2 (one-way ANOVA with LSD post-hoc test, $\mathrm{p}>0.05$ ), while by $60 \mathrm{~min}$ the homologous Symbiodinium B1 had released significantly more glycerol than had Symbiodinium E2 (one-way ANOVA with LSD post-hoc test, $\mathrm{p}>0.05$ ), and significantly more glucose than both Symbiodinium E2 and the heterologous Symbiodinium B1 (one-way ANOVA with LSD post-hoc test, $\mathrm{p}>0.005$ for both comparisons). 
Chapter 4
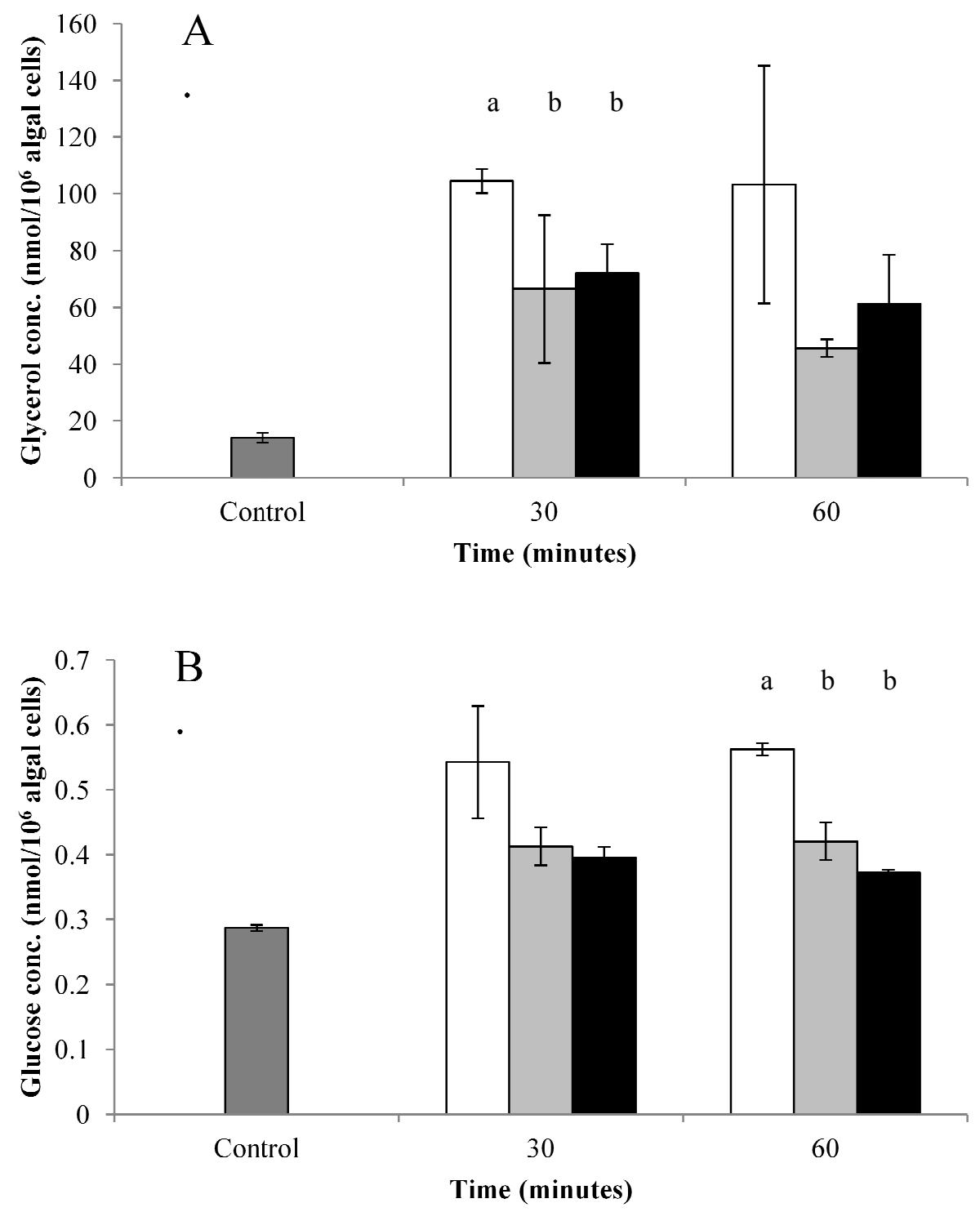

Figure 4.5. Concentration of (A) glycerol and (B) glucose in a host tissue homogenate of $A$. pulchella when incubated with different Symbiodinium types in the light. White bars, homologous Symbiodinium B1; grey bars, heterologous Symbiodinium B1; black bars, Symbiodinium E2. The dark grey bar represents the glycerol/glucose concentration in the host homogenate incubating medium at $0 \mathrm{~min}$ (control). At each time point, associations assigned different letters are significantly different from each other $(p<0.05)$. Values are means \pm SE; $n=3$ or 4 per time point. 
Chapter 4

\subsection{Discussion}

Overall, this study demonstrated that the presence of both homologous and heterologous symbiont types results in an increase in host tissue glycerol and glucose in the A. pulchella-Symbiodinium association although the accumulation patterns in the different associations of these two compounds varied. A significant increase in both metabolites was also observed when freshly-isolated Symbiodinium types were incubated in host tissue homogenate with homologous Symbiodinium B1 outperforming the other two types with regards to both glycerol and glucose release. Cell-specific metabolite concentrations revealed significant differences between the symbiont types with regards to their contribution to the glycerol and glucose pools, with the homologous Symbiodinium B1 contributing significantly more than the other two types and Symbiodinium E2 contributing the least. Given that the overall glycerol and glucose pools in the Symbiodinium B1 association (as demonstrated by the metabolite concentrations normalized against host protein content) were as high or higher than those in the symbiosis with Symbiodinium E2, it seems that a higher symbiont density (as in the case of type E2) can mask a relatively low contribution to the host metabolite pool per symbiont cell and vice versa.

\subsubsection{Glycerol and glucose levels in the host tissue}

A number of studies have investigated carbon dioxide fixation in Symbiodinium (Schmitz and Kremer, 1977; Trench, 1979; Streamer et al., 1993), though the identity of the compounds released and their quantities are still open to question (Davy et al. 2012). Of particular relevance to the current study, there is evidence for glycerol translocation in vitro, when freshly isolated Symbiodinium cells are incubated in a host tissue extract or "host release factor". (HRF; Grant et al., 1997, 1998), as well as in hospite in the sea anemone Condylactis gigantea (Battey and Patton, 1987). However, Ishikura et al. (1999) could not identify glycerol in hospite in the giant clam-Symbiodinium symbiosis, while Whitehead and Douglas (2003) could not detect it in the translocated material of the temperate sea anemone Anemonia viridis. A masters study by Hrdlicka (2000) that looked at metabolite transfer in the Aiptasia pallida-Symbiodinium association supported these findings. More recently, Burriesci 
et al. (2012) also did not find any evidence of glycerol transfer in symbioses with $A$. pulchella thus further suggesting that glycerol does not form a major part of the translocate. Like previous studies however, they did find that isolated Symbiodinium cells released glycerol and suggest that the explanation for this may be that production of glycerol by these algae may be part of a stress response. The evidence for glucose translocation is stronger however: for example both Ishikura (1999) and Whitehead and Douglas (2003) identified it in their studies with intact symbioses. Burriesci et al. (2012) supported these findings when they labled intact Aiptasia sp. in the light with ${ }^{13} \mathrm{C}$-bicarbonate and, after homogenization and separation of animal and algal fractions, found labelled glucose in the animal fraction. The current study is the first of its kind to not only measure both of these metabolites (i.e. glucose and glycerol) in the host tissues of a cnidarian-dinoflagellate symbiosis, but to also measure the effect of symbiont type on the internal pools of these metabolites. The study therefore adds to the evidence for both glycerol and glucose release in hospite, though the indirect nature of this evidence must still be acknowledged. Moreover, in all cases here the concentration of host tissue glucose was lower than that of glycerol, indicating that glycerol is likely to be the more significant metabolite present in the host tissue; this is consistent with the suggestion of Trench (1971a, b), based on radio-chromatographic studies in the temperate sea anemone Anthopleura elegantissima.

This study demonstrates that photosynthesis in the A. pulchella-Symbiodinium association results in an increased concentration of free glycerol and glucose in the host tissues, whatever symbiont type is present (except for the association with Symbiodinium E2 which did not demonstrate an increase in glycerol when normalized against host protein). However, the host-tissue concentrations of these two metabolites in the different symbioses were not always the same; for example, the symbiosis with Symbiodinium E2 contained higher levels of glycerol at 0 hours and in the early stages of the light incubation. Other studies have shown that hosts with certain Symbiodinium types grow faster than they do with others, which again suggests that they potentially receive a greater quantity of some or all metabolites from specific types (Fitt, 1985; Little et al., 2004). Little et al. (2004), for example, demonstrated that juveniles of the corals Acropora tenuis and A. millepora grow 2-3 
Chapter 4

faster when in symbiosis with Symbiodinium clade C than with clade D. However, in the current study the greater overall contribution of the symbiont population was not necessarily a reflection of the cell-specific contribution; the reasons and implications for this are discussed below.

While there was good evidence for both glycerol and glucose accumulation in the current study, the accumulation patterns of these two compounds differed. In particular, glycerol levels increased at first, but declined towards the end of the incubation period, while glucose levels only increased after several hours. The eventual decrease in glycerol may relate to its use for host metabolism. In particular, as suggested by Battey and Patton (1984), glycerol may be translocated to the host for short-term use as a respiratory substrate; these authors suggested that lipid droplets are more likely to be used during periods of reproduction and starvation. According to Battey and Patton (1987), host catabolism of glycerol is fairly rapid, with the turnover time of the glycerol pool in the sea anemone Condylactis gigantea estimated as 4 hours. The influence of host metabolism on the glycerol pool is supported by the elevated concentrations of glycerol in the presence of $\mathrm{NaCN}$, though it should be noted that cyanide would also have influenced respiration by the symbionts and can induce excessive photosynthate release to the host (Davy and Cook, 2001a). Cyanide inhibits respiration by binding to the terminal cytochrome in the electron transport system, which then blocks the donation of electrons to oxygen (Wishnick and Lane, 1969), however it can also inhibit photosynthesis through the binding to the ribulose-bis-phosphate (RuBP) carboxylase complex and the blocking of the incorporation of carbon dioxide (Wishnick and Lane, 1969). This latter effect could have contributed to the decrease in glycerol concentration between 30 and 60 min seen in all three symbioses, especially if the existing glycerol was lost through leakage to the surrounding seawater; this is possible given the ease with which glycerol diffuses through membranes.

The increase in host glucose concentration after 9 hours or more of light incubation was markedly different to the pattern seen with glycerol. The reason for this pattern is unclear, but possibilities include more rapid respiration of glucose by the host in the earlier stages of incubation or reduced glucose synthesis and/or translocation with 
Chapter 4

time; alternatively, the glucose increase may have resulted from the breakdown of other translocation products. Given that the anemones incubated with $\mathrm{NaCN}$ accumulated more glucose by 30 and $60 \mathrm{~min}$ than the non-inhibited anemones did by $60 \mathrm{~min}$, it seems that host respiration played a part in masking the true trend in glucose translocation (though the non-specific nature of $\mathrm{NaCN}$ and its potential impacts on carbon release should again be recognized).

\subsubsection{Carbon fluxes in isolated Symbiodinium}

The significant increase in the release of both glycerol and glucose in the presence of host tissue homogenate by all of the Symbiodinium types is consistent with the enhancement of photosynthate release seen in past studies (Muscatine 1967; Trench, 1971c; Muscatine et al., 1972; Muscatine et al., 1984; Hinde, 1988; Sutton and Hoegh-Guldberg, 1990; Gates et al., 1995; Grant et al., 1997, 1998; Withers et al., 1998; Ishikura et al., 1999; Davy and Cook, 2001a; Burriesci et al., 2012).This stimulation is thought to be due to the activity of a compound or combination of compounds present in the host tissues, referred to as a "host release factor" (HRF; Trench, 1971c; Muscatine et al., 1972; Hinde, 1988). The nature of this HRF is still uncertain (Davy et al, 2012) although studies by Grant et al. (1997; 1998, 2006b) suggest that it may be a water-soluble signalling molecule of $1000 \mathrm{kDa}$ or less. The amount of glycerol released by the homologous Symbiodinium B1 in host tissue homogenate was $104.4 \mathrm{nmol} / 10^{6}$ algal cells after $30-60 \mathrm{~min}$. This release rate is of a similar magnitude, though slightly higher than, that reported for Symbiodinium cells isolated from the scleractinian coral Plesiastrea versipora which released 14-46 nmol glycerol $/ 10^{6}$ algal cells when incubated in a host-tissue extract of this same species for 4 hours in the light (Grant et al., 1997). Burriesci et al. (2012) found no detectable glucose release by freshly-isolated Symbiodinium and no comparable data from other studies are available for glucose.

The results with the isolated algae demonstrate that the homologous Symbiodinium B1 outperformed the other two types with regards to glycerol and glucose accumulation/release. It should be noted, however, that since HRF studies are conducted in vitro, with isolated dinoflagellates incubated in homogenised host 
Chapter 4

tissue, they are not representative of theenvironment in the intact symbiosis and it is possible that HRF is an experimental artifact (Davy et al., 2012). For example, translocation patterns in response to host (Aiptasia pallida) starvation when the symbionts were either exposed in vitro to HRF or retained in hospite were found to differ (Davy and Cook 2001a, b).

\subsubsection{The role of metabolite transfer in host-symbiont recognition and specificity}

While the protein-specific concentrations of glycerol and glucose provide insight into the nutritional potential of the different symbioses, the cell-specific response is far more informative with respect to the possible roles of metabolite exchange in hostsymbiont recognition and specificity. Indeed, the two measures were shown to be quite different, reflecting the fact that a high symbiont density can mask a relatively low contribution to the host metabolite pool per symbiont cell and vice versa. For example, when in symbiosis with Symbiodinium E2, the individual symbionts contributed relatively little yet the overall glycerol and glucose pools were as high or higher than those in the symbiosis with the homologous Symbiodinium B1 because of the higher density of symbionts in the Symbiodinium E2 association. As described in Chapter 2, this high density of Symbiodinium E2 may indicate an overshooting of the 'steady state' due to the loss of control by the host of the symbiont population. Little is known of the mechanisms that underlie host-symbiont recognition, integration and the regulation of host-symbiont biomass, however it may well be that metabolite signals play a role. If so, then quantitative and qualitative aspects of the translocated photosynthate could signal to the host that it has acquired a compatible symbiont type, as they do in the green Hydra symbiosis where maltose release is required for successful symbiosis establishment (McAuley, 1982; McAuley and Smith, 1982). However, the disparity in the current study between the concentrations of glycerol and glucose in the presence of the different symbiont types and their infection success does not support this; though we cannot exclude roles for other mobile compounds. 
Chapter 4

One possibility is that Symbiodinium E2 was maintained at a higher density to meet the nutritional demands of the host, though we currently know too little about the regulation of symbiont biomass by the host (and vice versa) to know what the basis for this would be (Weis et al., 2008; Davy et al., 2012). It is of note, though, that Symbiodinium E2 was originally cultured from a single free-living cell collected from Wellington Harbour (New Zealand), which may explain its unusual behaviour when in hospite. There is increasing evidence that such free-living Symbiodinium types are common in the field (Takabayashi et al., 2012) and these results would suggest that they may perform differently when in hospite than symbiotic Symbiodinium. It is also important to note that, while Symbiodinium E2 overcame its limited cell-specific potential to enhance the metabolite pools of the host anemone by reaching a higher density, there may be energetic costs to the host of maintaining this higher density. Indeed, when the contribution of the symbiotic algal population to host respiration is calculated, the homologous and heterologous Symbiodinium B1 appear to be more effective than Symbiodinium E2 at supporting the host's metabolic needs due to differences in the respiratory rate (Chapter 2).

From this study it is clear that symbiont type has an effect on host tissue glycerol and glucose pools, however it remains to be seen whether this is a result of the increased translocation of these metabolites to the host, or simply the catabolism of other translocated products. Further work looking into metabolite translocation using more sophisticated approaches such as metabolite profiling (see review by Davy et al., 2012) in associations with different symbiont types is needed, as is further research on the potential roles of mobile compounds in host-symbiont recognition. 
Chapter 5

\section{Chapter 5: General discussion}

This thesis examined the relationship between the physiological diversity of different symbiont types and host-symbiont specificity in the model system Aiptasia pulchella. Each chapter focused on a different physiological aspect: Chapter 2, the ability of different symbiont types to meet the host's carbon demands; Chapter 3, how symbiont type effects host cell $\mathrm{pHi}$ and, in turn, how pHi effects the photosynthetic rate of different symbiont types when in hospite; and Chapter 4, how symbiont type impacts the quality and quantity of metabolites translocated from symbiont to host. A wide range of symbiont types was profiled initially (Chapter 2), namely Symbiodinium A1.4, both homologous and heterologous Symbiodinium B1, Symbiodinium E2 and Symbiodinium F5.1, before the more focused study of three types (homologous and heterologous Symbiodinium B1 and Symbiodinium E2) in Chapters 3 and 4.

The main findings were that: (1) Different symbiont types exhibit different proliferation rates when inside the host; (2) Symbiont type impacts the photosynthetic and respiratory attributes of the symbiosis which, in turn, affect the nutritional potential of the symbiosis; (3) Different Symbiodinium types have different $\mathrm{pH}$ optima, which do not necessarily match the pHi of the host cell and could lead to differential photosynthetic performances when inside the same host species; and (4) Symbiont type has an effect on the release of glycerol and glucose to the host, and the accumulation of these energy-rich compounds in the host's tissues. Here, the interrelationships between these findings will be discussed, as will their implications for our understanding of host-symbiont specificity and the potential for the establishment and persistence of novel associations in the field. 
Chapter 5

\subsection{Mutualism vs parasitism}

\subsubsection{Shift along the symbiosis continuum}

Although symbiotic relationships can be divided into mutualistic, commensal and parasitic interactions, many symbioses do not fit neatly into a single category (Douglas, 1994). Thus it is more accurate to describe symbiosis as a continuum that spans from parasitism to mutualism (Dimijian, 2000) with a given relationship being able to shift along the continuum from one end to the other. Past research suggests that algae can be costly to hosts, potentially acting as parasites. Douglas and Smith, (1983) demonstrated that when hydras that host Chlorella algae were grown in the dark, uninfected hosts were found to outgrow infected hosts. Cnidariandinoflagellate symbioses have been defined as being mutualistic interactions with the algae benefiting their hosts (Trench, 1993). Numerous cnidarian hosts, however, acquire symbiotic algae infectiously at each sexual generation (Fadlallah, 1983) and it is believed that the horizontal transmission of symbionts promotes the evolution of parasitism (Fine, 1975; Ewald, 1983; Bull, 1994). Muller-Parker and Davy (2001) described that, in some environments, algal symbionts grow and proliferate within hosts during winter despite there being minimal potential for photosynthesis. The evidence generated from this thesis indicates that the cnidarian-dinoflagellate symbiosis may not function strictly as a mutualistic interaction and may, under certain situations, become parasitic. The heterologous symbiont, Symbiodinium E2, displayed the highest rate of proliferation inside the host, overshooting the 'preferred' steady state for the symbiosis. This suggests an inability of re-infected $A$. pulchella to effectively regulate these algal populations. This opportunistic behaviour has been previously noted in clade A Symbiodinium present in corals recovering from bleaching events (Toller et al., 2001b; LaJeunesse, 2005). Also, in the scyphozoan, Cassiopea xamachana, acquisition of symbionts from the external environment, resulted in a rapid proliferation of these symbionts inside the host as well as a corresponding reduction in host growth rate (Sachs and Wilcox, 2006). The data from this thesis suggest a similar outcome when Symbiodinium E2 infects A. pulchella. A clear trend throughout the study was the marked difference in the behaviour of Symbiodinium B1 and E2 when in association with A. pulchella. This 
Chapter 5

was demonstrated by the earlier onset of a fully autotrophic state (with respect to carbon) and the higher CZAR values achieved in the symbioses hosting both the homologous and heterologous Symbiodinium B1 than type E2. Symbiodinium E2 also demonstrated a lower cell-specific release of glycerol and glucose, and associations with this type displayed lower levels of both metabolites in the host tissue metabolite pools. Symbiodinium types have been previously shown to differ with respect to the amount of carbon they release. Carbon fixation studies by Stat et al. (2008), for example, indicated that clade A Symbiodinium may not provide as much carbon to the host as clade C Symbiodinium and thus may not meet the host's nutritional requirements. This reduced contribution of carbon by the symbiont to the host may lead to a decline in fitness of the coral host and, in turn, the carbon retained by the symbiont may increase algal fitness and allow for rapid algal proliferation (as evidenced in the A. pulchella-Symbiodinium E2 association in this study). Stat et al. (2008) demonstrated that Acropora cytherea from Hawaii with clade A Symbiodinium exhibited suboptimal health states and an increased incidence of disease when compared to corals sampled on the same reef that harboured clade $\mathrm{C}$ Symbiodinium. Thus the acquisition of clade A Symbiodinium by A. cytherea may lead to a reduction in coral health or, alternatively, these opportunistic symbiont types may proliferate in health-compromised corals (Stat et al., 2008). Similarly, in the wild, it is possible that due to its ability to rapidly proliferate in A. pulchella, Symbiodinium E2 may be capable of out-competing other symbiont types. However, as suggested by this $\mathrm{PhD}$ study, the interaction of A. pulchella with Symbiodinium E2 may not be mutually beneficial and a shift to parasitism is indicated. A shift from mutualism to parasitism has been previously demonstrated by Sachs and Wilcox (2006), in the symbiosis between the scyphozoan C. xamachana and Symbiodinium. They showed that horizontally transmitted algae proliferated faster within hosts and had higher dispersal rates from hosts than vertically transmitted algae. However, the horizontally transmitted algae reduced host reproduction and growth. Steen (1986) demonstrated the parasitic nature of some zooxanthellae when he exposed $A$. pulchella, previously kept in the dark, to cultured zooxanthellae under photosynthesizing conditions. He observed that, after the establishment of a stable symbiosis, the symbiotic animals had a protein biomass significantly lower than did control aposymbiotic animals, suggesting that the reinfection of the symbiotic animals by zooxanthellae caused a decrease in animal biomass. He also showed that 
aposymbiotic anemones starved in darkness suffered a lower mortality rate than symbiotic anemones under the same conditions, suggesting that zooxanthellae may impose a fatal metabolic burden on their hosts. Thus under certain circumstances, normally beneficial symbionts may become harmful, and thus a symbiont's status of being either a mutualist or a parasite is by no means stable.

\subsubsection{Free-living Symbiodinium}

The phylogenetic positioning of both clades $\mathrm{A}$ and $\mathrm{E}$ has been found to be either basal or sister to the other Symbiodinium clades (Carlos et al., 1999; Baillie et al., 2000; LaJeunesse, 2001; Pochon et al., 2004; Takabayashi et al., 2004; Chen et al., 2005; Stat et al., 2008). This positioning suggests that these clades were, either the first groups to initiate a symbiosis with invertebrates, or that they evolved from the ancestral dinoflagellate that first formed a symbiosis with invertebrates and are divergent lineages on a different evolutionary path to other Symbiodinium clades (Stat et al., 2008). Alternatively, it is possible that a free-living state is ancestral within the genus and a symbiotic lifestyle evolved independently in the two sister lineages. It certainly appears that the more derived Symbiodinium clades such as B and $\mathrm{C}$ are better able to form a mutualistic interaction with their hosts (e.g. the nutritional advantage of $A$. pulchella hosting Symbiodinium B1 demonstrated in this study). Clade E Symbiodinium is extremely rare and has only been identified in the temperate anemone Anthopleura elegantissima (LaJeunesse, 2001). This rarity indicates that Clade $\mathrm{E}$ either does not form symbioses readily, is becoming extinct, or it is an opportunistic dinoflagellate that is more abundant as a free-living entity (Stat et al., 2008). The Symbiodinium E2 used in this PhD study was originally isolated from Wellington Harbour (NZ) and its genotype does not match that of any known symbiotic dinoflagellates from New Zealand which belong to Symbiodinium Clade A (J. Howe, Victoria University of Wellington, pers. comm.). Therefore, it is suspected that it may be free-living in nature. Since the $\mathrm{pH}$ of seawater is approximately 8.1, the superior performance of Symbiodinium E2 at a $\mathrm{pH}$ of 8 (as evidenced in this study) provides further proof that it may be suited to a free-living environment. As more information is coming to light about the higher-than-expected incidence of free-living Symbiodinium (Takabayashi et al., 2012), so it is becoming more likely that the symbiotic state may not be the preferred state for all Symbiodinium types and 
Chapter 5

that, in fact, a large proportion of the genus may not be physiologically suited to symbiosis but rather to a free-living lifestyle

\subsection{Will corals adapt to climate change?}

\subsubsection{Symbiont type and the infection process}

This $\mathrm{PhD}$ study provided some insight into the effect that the acquisition of different symbiont types has on the infection process as well as the short and long-term maintenance of a stable association. Given that all the symbiont types (i.e. Symbiodinium A1.4, E2, F5.1 and both the homologous and heterologous B1) tested were taken up by $A$. pulchella, it is clear that this host can form an association with a wide range of symbiont types. Moreover, there was an apparent lack of discrimination by the host during the early stages of infection, at least with these symbiont types. Discrimination by the host between homologous and heterologous symbionts has in past studies been found to occur early in infection (Jolley and Smith, 1980; Schoenberg and Trench, 1980b; Weis et al., 2001; Belda-Baillie et al., 2002; Rodriguez-Lanetty et al., 2004; Markell and Wood-Charlson, 2010), however it has also been noted later in host development (Coffroth et al., 2001; Little et al., 2004; Gómez-Cabrera et al., 2008). The basis of this specificity is poorly understood, however inter-partner signalling has been shown to play an important role both in symbiont uptake (Coffroth et al., 2001; Belda-Baillie et al., 2002; Rodriguez-Lanetty et al., 2003; Logan et al., 2010) and symbiosis establishment (Chen et al., 2003; Chen et al., 2004; Chen et al., 2005), and some form of cellular signalling could also explain why the homologous B1 provided the host with significantly more glycerol and glucose than the heterologous B1 type and Symbiodinium E2, and ultimately contributed more to the host's metabolic carbon demands. It has been suggested that host release factor (HRF), which is present in homogenised host tissue, acts as a signal molecule and mediates the release of photosynthetically-fixed carbon by the symbiont (Trench, 1971c; Muscatine et al., 1972; Muscatine et al., 1984; Hinde, 1988; Sutton and Hoegh-Guldberg, 1990; Grant et al., 1997; Davy and Cook, 2001a). Given that homologous Symbiodinium B1 released proportionally more glucose and 
Chapter 5

glycerol in response to a host tissue homogenate than did heterologous Symbiodinium B1 and Symbiodinium E2, it would appear that this signal is symbiont-specific. HRF studies are not representative of the environment in the intact symbiosis however, and the HRF may be an experimental artifact (Davy et al., 2012). Also, at this stage the information about symbiosis-specific signal molecules is limited. As more becomes known about the host genome, so the identification of candidate signal molecules will progress (Davy et al., 2012).

The symbionts acquired also need to be compatible with the host cell intracellular environment. The intracellular environment of the host may also play a part in determining host-symbiont specificity; for example, pHi may influence symbiont function, as shown in this thesis. Host cell $\mathrm{pHi}$ has been shown to influence various facets of cell metabolism including the functioning of membrane channels and enzymes, as well as intracellular signalling (Busa and Nucitelli, 1984; Busa, 1986; Venn et al., 2009), so changes in pHi could influence the communication pathways between symbiont and host. Data from this study indicate that all the symbionts tested were functional at the intracellular $\mathrm{pH}$ in A. pulchella. However, for both homologous Symbiodinium B1 and Symbiodinium E2, the optimal ambient $\mathrm{pH}$ for photosynthesis did not coincide with the host-cell pHi measured in A. pulchella harbouring those types. This indicates that the situation is far more complex. Many other factors that are important for promoting a functional symbiosis need to be considered such as the functioning of membrane channels and enzymes and intracellular signalling. (Davy et al., 2012).

The ability of a symbiont to proliferate inside the host and increase its population size is critical for the formation of a stable association. However, the regulation of the symbiont population is equally important. If the symbiont density is too low then the symbiont population might be diluted and hence be less effective at supporting the host's metabolic needs (Neckelmann and Muscatine, 1983; Taylor et al., 1989), however excessive replication of the symbiont could turn out to be costly to the host and result in higher virulence (Sachs and Wilcox, 2006; Stat et al., 2008). This thesis demonstrated how critical it is for the host to be able to regulate its symbiont population. Symbiodinium A1.4, F5.1 and E2 were able to attain higher densities inside the host than Symbiodinium B1, however this rapid proliferation came at a cost 
Chapter 5

as demonstrated by the higher metabolic rates seen in the anemones infected with Symbiodinium E2 and F.51, as well as the less favourable P:R ratios and CZAR values of associations with types A1.4, F5.1 and E2. Cnidarians regulate their symbiont populations by controlling algal division rates, or by selectively destroying or expelling unwanted symbionts (Gates et al., 1992; Falkowski et al., 1993; Baghdasarian and Muscatine, 2000; Dunn et al., 2002; Dunn and Weis, 2009; Davy et al., 2012). It appears that $A$. pulchella is unable to effectively regulate these heterologous symbiont populations, possibly due to the inhibition of some of the above mentioned mechanisms. How this may occur is uncertain though as currently we do not know enough about the underlying cellular process involved (Davy et al., 2012).

Metabolic exchange is central to the ecological success of the coral-dinoflagellate symbiosis (Muscatine, 1990) as, given that most coral reefs are found in nutrient poor waters (Dubinsky, 1990), the symbiont needs to be able to provide the host with enough carbon to meet its host's respiratory demands in order for the symbiosis to persist (Muscatine, 1990). The overall findings from this thesis suggest that it is Symbiodinium B1 or, more specifically, homologous Symbiodinium B1, that provides the greatest nutritional advantage to A. pulchella both in terms of meeting the host's carbon demands, and providing the highest concentration of two key metabolites, glucose and glycerol. This may be facilitated through effective communication pathways between the homologous symbiont and its host, which may not function optimally when a heterologous symbiont is present. Again, more information is necessary about the underlying cellular process involved in order to speculate further (Davy et al., 2012).

\subsubsection{The implications on the recovery of corals from bleaching events}

It has been proposed that corals are able to switch their symbionts as an adaptation to changing environmental conditions (the "adaptive bleaching hypothesis"; Buddemeier and Fautin, 1993). Thus, following bleaching events, the formation of novel symbioses could assist in coral reef recovery. As shown in this thesis, $A$. pulchella is able to form an association with a range of symbiont types, with some of the heterologous types even exhibiting greater proliferation rates inside the host than 


\section{Chapter 5}

the homologous types. This indicates that, should coral associations behave in a similar manner in the field, then they may also have the capacity to form novel symbioses. However, as mentioned previously, the ability to form an association is not the entire picture. In order for a functional association to persist, the symbiont needs to be able to meet the host's nutritional needs (Muscatine, 1990). The findings from this thesis indicate that there are metabolic costs associated with hosting heterologous symbionts and that, should a novel association form, the new symbiont type may not necessarily be as nutritionally advantageous as the homologous type, possibly leading to reduced growth and success of the association. Differences in the growth and survivorship of symbiotic hosts with different symbiont types have been noted in other studies (Fitt, 1985; Little et al., 2004). Thus, it is likely that, should a novel symbiosis arise after a bleaching event, it may function less successfully than the original association (at least under non-stressful conditions). Clearly then, whether corals are able to form novel associations in the field is not the only concern with respect to their capacity to recover from bleaching events; the type of symbiont they associate with, is equally important. However, the nutritional contribution of the symbiont may not be of as much significance if the coral is able to rely on heterotrophy to supplement its carbon demands. A recent study by Houlbrèque and Ferrier-Pagès (2008) suggested that heterotrophy is significant to the energy budget of corals and contributes up to $70 \%$ of daily carbon requirements when feeding on all possible food sources is taken into account. Moreover, corals have been shown to upregulate heterotrophic feeding when photosynthesis is suppressed (Hoogenboom et al., 2010), either due to decreased water clarity (Anthony and Fabricius, 2000) or when symbionts are lost from coral tissue (Grottoli et al., 2006). Therefore, a reduced carbon contribution of the resident symbiont population may not be of great importance should the coral be able to compensate for this through heterotrophy. This compensatory mode is particularly important in temperate symbioses as temperate corals are thought to be more heterotrophic than tropical corals (FitzGerald and Szmant, 1988; Tremblay et al., 2011).

Support for the "adaptive bleaching hypothesis" is lacking though, and despite some corals being capable of acquiring symbionts from the water column, this acquisition has been found to be temporary as these infections are not maintained as stable symbioses (Coffroth et al., 2010). It is more likely that a shift in the dominant 
Chapter 5

member of a mixed symbiont population will occur as a result of bleaching rather than the formation of a novel association (Baker, 2001; Baker et al., 2004; Berkelmans and van Oppen, 2006; Mieog et al., 2007; Jones et al., 2008). The findings from this thesis apply to the "symbiont shuffling" hypothesis too however, as it still involves a change in the dominant symbiont type. Thus a shift to a less nutritionally advantageous symbiont type may negatively impact the host. For example, Symbiodinium clade D is found in some corals that have survived episodes of severe bleaching and is thus considered to be a thermally-tolerant type (Baker et al., 2004; Rowan, 2004; Stat and Gates, 2011). Little et al. (2004), however, demonstrated that juveniles of the corals Acropora tenuis and Acropora millepora grow 2-3 faster when in symbiosis with Symbiodinium clade C than with clade D indicating that, despite the advantage of hosting type D2 during bleaching events, prolonged association with this type may be disadvantageous for the growth and persistence of the symbiosis. In support of this, Thornhill et al. (2005) demonstrated shifts in the dominant symbiont type present in certain colonies of Montastrea annularis and Montastrea franksi from the Florida Keys as a result of recovery from the stresses of the 1997-1998 El Niño Southern Oscillation (ENSO) event. Over several years, these authors found that stress-tolerant Symbiodinium clade D was progressively replaced in these colonies by other symbiont types. This suggests that certain corals may "shift" their algal population to a more stress resistant Symbiodinium type during periods of coral bleaching but then reinstate the more nutritionally advantageous symbiont as the dominant type during bleaching recovery. This indicates that the type of symbiont that the coral "shifts" to during the bleaching period may not be of much significance as, even if it does not meet the coral's carbon demands, its contribution may be enough to allow the coral to survive during this period before the more nutritionally advantageous symbiont is reinstated. Such a scenario is even more applicable given that corals appear to be able to supplement their carbon requirements through heterotrophy (Grottoli et al., 2006; Hoogenboom et al., 2010; Tremblay et al., 2011; Wijgerde et al., 2011). 
Homologous Symbiodinium B1

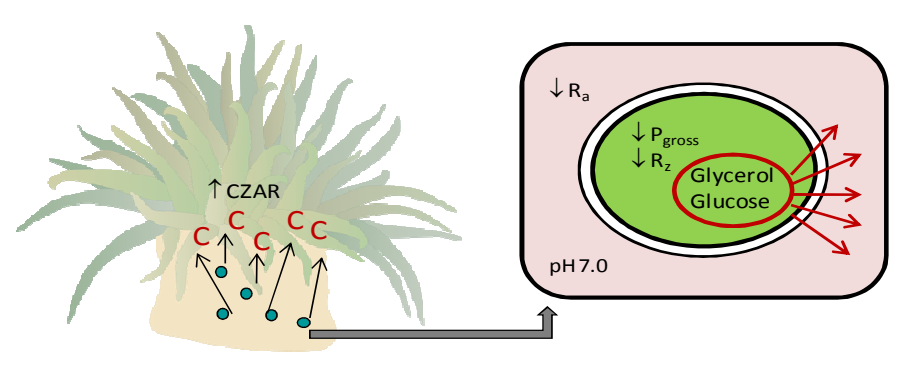

Heterologous Symbiodinium B1

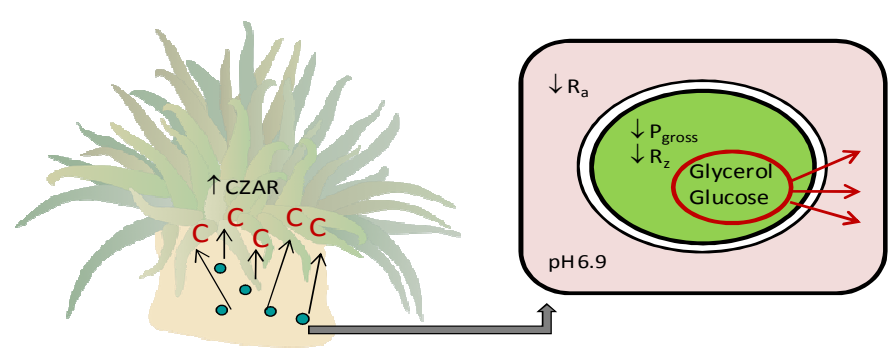

Symbiodinium E2

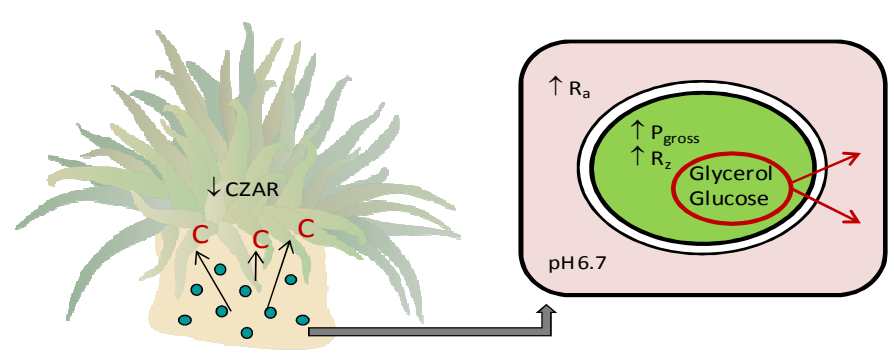

Lower algal cell

density

Lower total $\mathrm{P}_{\text {gross }}$

Lower $\mathrm{R}_{\mathrm{a}}$

Higher P:R

Higher CZAR

Higher metabolite translocation

Higher pHi

Lower algal cell

density

Lower total $\mathrm{P}_{\text {gross }}$

Lower $\mathrm{R}_{\mathrm{a}}$

Higher P:R

Higher CZAR

Moderate metabolite translocation

Higher pHi

Higher algal cell density

Higher total $\mathrm{P}_{\text {gross }}$

Higher $\mathrm{R}_{\mathrm{a}}$

Lower P:R

Lower CZAR

Lower metabolite translocation

Lower $\mathrm{pHi}$

Figure 5.1. Schematic diagram illustrating the summary of the physiological differences and/or similarities between the A. pulchella-Symbiodinium associations investigated in this study. The physiological parameters compared include: algal cell density (represented by the green circles inside the anemone diagram), total $\mathrm{P}_{\text {gross }}$ (gross photosynthetic rate), $\mathrm{R}_{\mathrm{a}}$ (host respiration), $\mathrm{P}: \mathrm{R}$ (ratio of $\mathrm{P}_{\text {gross }}$ to $\mathrm{R}_{\mathrm{a}}$ ), $\mathrm{R}_{\mathrm{z}}$ (zooxanthellar respiration), CZAR (contribution of translocated zooxanthellar carbon to the host's daily respiratory carbon requirements), metabolite translocation and host cell pHi. The terms "lower" and "higher" are relative terms based on statistical analyses between the three symbioses. 
Chapter 5

\subsection{Future work}

While the findings from this work indicate that the physiology of a symbiosis is symbiont type dependent, these studies only incorporated a small number of different Symbiodinium types. In order to get a clearer picture of the diversity in symbiosis physiology, a wider range of types needs to be investigated. Of equal importance, the above studies were all laboratory based and do not give a true representation of the behaviour of cnidarian-dinoflagellate symbioses in the field. Also, the studies were performed using the anemone, A. pulchella, and despite its applicability as a model system for corals, the contribution of zooxanthellar carbon to the host's respiratory requirements has been shown to vary, both between cnidarian hosts and with environmental parameters (McCloskey and Muscatine, 1984; Davy et al., 1996; Muller-Parker and Davy, 2001). It is therefore imperative to extend this type of work to corals in the field, to determine how these associations behave in nature.

Because the symbiosome membrane complex acts as a barrier between the host cell cytoplasm and the symbiont, an understanding of the communicative processes between the algal symbiont and its cnidarian partner, which are no doubt crucial to the nutrition, stability and persistence of the symbiotic relationship, is necessary. Despite recent attempts to identify some of the symbiosome membrane proteins involved in membrane formation, host-symbiont signalling, molecular transport and recognition, photosynthesis, and host and symbiont cell cycle regulation (Peng et al., 2010), more information is still necessary to comprehensively understand the interaction between the host and symbiont. In order to be able to elucidate how symbiont type affects the communication pathways between the symbiont and host (i.e. how, for example, a heterologous symbiont may negatively impact these pathways), insight into the influence of different host-symbiont pairings on membrane function as well as the host cell intracellular environment (e.g. the host cell pHi) is needed. Expanding our knowledge of symbiosome membrane composition will in turn provide much needed information about inter-partner signalling, and once the membrane proteins involved are identified, their function in associations with different symbiont types can be studied. Moreover, the use of modern chromatographic and mass spectrometric techniques, as well as comparative 
Chapter 5

metabolic profiling to characterize the metabolites transferred between partners during different phases of the symbiosis and with different host-symbiont combinations, will provide great insight both into host nutrition and inter-partner signalling (Davy et al., 2012). Finally, since a number of free-living Symbiodinium types have now been identified (Takabayashi et al., 2012), it would be interesting to determine if some of these types can be taken up by A. pulchella and are able to form a symbiosis and secondly, determine how they behave when in hospite with respect to the quantity and quality of carbon production, symbiosome membrane function and host cell pHi.

\subsection{Conclusion}

Evidence that corals are able to host multiple symbiont clades has increased in recent years. By surveying 26 coral species previously thought to be restricted to hosting a single Symbiodinium clade ('symbiotic specialists'), Silverstein et al. (2012) showed that the ability to associate with multiple symbiont clades is common in scleractinian corals. This finding provides further indication that reef corals may be able to adapt or acclimatize to environmental change via shifts in the dominant resident symbiont population (symbiont "shuffling"). Findings from the current study suggest that corals may be able to maintain an association with a range of Symbiodinium types and so, should a symbiont population shift occur within a coral as a result of bleaching, the new, dominant symbiont type will be able to successfully populate the host. However, the new symbiont type may not be as nutritionally advantageous as the original type which could have implications for the growth and survivorship of the coral, unless it is able to supplement its carbon demands heterotrophically. Given however, that corals have been shown to upregulate heterotrophic feeding when photosynthesis is suppressed (Grottoli et al., 2006; Hoogenboom et al., 2010; Tremblay et al., 2011) and to reinstate the more nutritionally advantageous symbiont as the dominant type during bleaching recovery, the reduced carbon contribution of the "interim" symbiont type may not be a major concern. Furthermore, it is likely that the metabolic cost associated with hosting the "interim" symbiont type as the dominant type may be compensated for by its rapid proliferation ability (as 
Chapter 5

demonstrated by some of the heterologous symbiont types tested in this study). The reason being is that, should the "interim" symbiont be more stress tolerant, its ability to rapidly populate the host would clearly be of benefit to the host during periods of bleaching. Finally, the rapid proliferation demonstrated by heterologous types and the associated metabolic cost to the host, could be an indication of the opportunistic nature of these types and may indicate an inability to form mutualistic associations; this is a fascinating area for future research. 


\section{References}

Abrego, D., Ulstrup, K. E., Willis, B. L., van Oppen, M. J. H. (2008). Speciesspecific interactions between algal endosymbionts and coral Hosts define their bleaching response to heat and light stress. Proc. R. Soc. Lond. B Biol. Sci. 275, 2273 -2282 .

Allemand, D., Furla, P., Bénazet-Tambutté, S. (1998). Mechanisms of carbon acquisition for endosymbiont photosynthesis in Anthozoa. Can. J. Bot. 76, $925-$ 941.

Anthony, K. R. N. and Fabricius, K. E. (2000). Shifting roles of heterotrophy and autotrophy in coral energy budgets at variable turbidity. J. Exp. Mar. Biol. Ecol. 252, $221-253$.

Armstrong, J. A. and Hart, P. D. (1975). Phagosome-lysosome interactions in cultured macrophages infected with virulent tubercle bacilli, J. Exp. Med. 142, 1 16.

Baghdasarian, G. and Muscatine, L. (2000). Preferential expulsion of dividing algal cells as a mechanism for regulating algal-cnidarian symbiosis. Biol. Bull. 199, $278-$ 286.

Baillie, B., Belda-Baillie, C., Maruyama, T. (2000). Conspecificity and Indo-Pacific distribution of Symbiodinium genotypes (Dinophyceae) from giant clams. J. Phycol. 36, $1153-1161$.

Baker, A. C. (1999). The symbiosis ecology of reef-building corals. PhD dissertation, Univ. of Miami.

Baker, A. C. (2001). Ecosystems: Reef corals bleach to survive change. Nature 411, $765-766$. 


\section{References}

Baker, A. C. (2003). Flexibility and specificity in coral-algal symbiosis: diversity, ecology, and biogeography of Symbiodinium. Annu. Rev. Ecol. Evol. Syst. 34, $661-$ 689.

Baker, A. C., Starger, C. J., McClanahan, T. R., Glynn, P. W. (2004). Corals' adaptive response to climate change. Nature 430, 741.

Ball, E. G. and Stock, C. C. (1937). The pH of sweater as measured with the glass electrode, Biol. Bull., 73, 221 - 226.

Barbieri, M. A., Li, G. Colombo, M. I., Stahl, P. D. (1994). Rab5, an early acting endosomal GTPase, supports in vitro endosome fusion without GTP hydrolysis. $J$ Biol. Chem. 269, 18720 - 18722.

Barneah, O., Benayahu, Y., Weis, V. M. (2006). Comparative proteomics of symbiotic and aposymbiotic juvenile soft corals, Mar. Biotechnol. 8, $11-16$.

Battey, J. F. and Patton, J. S. (1984). A reevaluation of the role of glycerol in carbon translocation in zooxanthellae-coelenterate symbiosis. Mar. Biol. 79, 27 - 38.

Battey, J. F. and Patton, J. S. (1987). Glycerol translocation in Condylactis gigantea. Mar. Biol. 95, $37-46$.

Bay, L. K., Nielsen, H. B., Jarmer, H., Seneca, F., vanOppen, M. J. H. (2009). Transcriptomic variation in a coral reveals pathways of clonal organisation. Marine Genomics 2, $119-125$.

Bay, L. K., Cumbo, V. R., Abrego, D., Kool, J. T., Ainsworth, T. D., Willis, B. L. (2011). Infection dynamics vary between Symbiodinium types and cell surface treatments during establishment of endosymbiosis with coral larvae. Diversity 3, 356 -374 . 
References

Belda-Baillie, C. A., Silvestre, V., Villamor, K., Monje, V., Gomez, E. D., Baillie, B. K. (1999). Evidence for changing symbiotic algae in juvenile tridacnids. J. Exp. Mar. Biol. Ecol. 241, 207 - 221.

Belda-Baillie, C. A., Baillie, B. K. and Maruyama, T. (2002). Specificity of a model cnidarian- dinoflagellate symbiosis. Biol. Bull. 202, $74-85$.

Berkelmans, R. and van Oppen, M. J. H. (2006). The role of zooxanthellae in the thermal tolerance of corals a 'nugget of hope' for coral reefs in an era of climate change. Proc. R. Soc. Lond. B. Biol. Sci. 273, $2305-2312$.

Bil, K. Y., Kolmakov, P. V., Parnik, T., Titlyanov, E. A., Muscatine, L. (1991). Photosynthetic products in zooxanthellae of the symbiotic coral Stylophora pistillata and Seriatopora coliendrum situated at various depths. Fiziol. Rast. (Moscow), 38, $846-854$.

Blank, R. J. and Muscatine, L. (1987). How do combinations of nutrients cause symbiotic Chlorella to overgrow Hydra? Symbiosis 3, 123-134.

Blanquet, R. S., Nevenzel, J. C., Benson, A. A. (1979). Acetate incorporation into the lipids of the anemone Anthopleura elegantissima and its associated zooxanthellae. Mar. Biol. 54, 185 - 194.

Bolte, S., Talbot, C., Boutte, Y., Catrice, O., Rea, N. D., Satiat-Jeunemaitre B. (2004). FM-dyes as experimental probes for dissecting vesicle trafficking in living plant cells. J. Microsc. (Oxf.) 214, $159-173$.

Bossert, P. and Dunn, K. W. (1986). Regulation of intracellular algae by various strains of symbiotic Hydra viridissima. J. Cell Sci. 85, 187 - 195.

Brown, B. E. (1997). Coral bleaching, causes and consequences. Coral Reefs 16, 129 -138 . 


\section{References}

Bucci, C., Parton, R. G., Mather, I. H., Stunnenberg, H., Simons, K., Hoflack, B., Zerial, M. (1992). The small GTPase rab5 functions as a regulatory factor in the early endocytic pathway. Cell 70, $715-728$.

Bucci, C., Thomsen, P., Nicoziani, P., McCarthy, J., van Deurs, B. (2000). Rab7: a key to lysosome biogenesis, Mol. Biol. Cell. 11, $467-480$.

Buckler, K. J. and Vaughan-Jones, R. D. (1990). Application of a new pH-sensitive fluoroprobe (carboxy-SNARF-1) for intracellular $\mathrm{pH}$ measurement in small, isolated cells. Pflüg. Arch. Eur. J. Phy. 417, $234-239$.

Buddemeier, R. W. and Fautin, D. G. (1993). Coral bleaching as an adaptive mechanism. BioScience 43, 320 - 326.

Buddemeier, R. W., Baker, A. C., Fautin, D. G., Jacobs, J. R. (2004). The adaptive hypothesis of bleaching. In Rosenberg, E. and Loya, Y. (ed.), Coral health and disease. Berlin, Germany, Springer-Verlag.

Bull, J. (1994). Perspective: Virulence. Evolution (Lawrence, Kans.) 48, 1423 - 1437.

Burriesci, M. S., Raab, T. K., Pringle, J. R. (2012). Evidence that glucose is the major transferred metabolite in dinoflagellate-cnidarian symbiosis. J. Exp. Biol. 215 : $3467-3477$.

Busa, W. and Nucitelli, R. (1984). Metabolic regulation via intracellular pH, Am. J. Physiol. 246, R409 - R438.

Busa, W. (1986). Mechanisms and consequences of pH-mediated cell regulation. Annu. Rev. Physiol. 48, $389-402$.

Caldeira, K. and Wickett, M. E. (2003). Anthropogenic carbon and ocean pH, Nature 425,365 . 
Cantin, N., van Oppen, M. J. H., Willis, B. L., Mieog, J. C., Negri, A. P. (2009). Juvenile corals can acquire more carbon from high-performance algal symbionts. Coral Reefs 28, $405-414$.

Carlos, A., Baillie, B., Kawachi, M., Maruyama, T. (1999). Phylogenetic position of Symbiodinium (Dinophyceae) isolates from tridacnids (Bivalvia), cardiids (Bivalvia), a sponge (Porifera), a soft coral (Anthozoa), and a free-living strain. J. Phycol. 35, $1054-1062$.

Cernichiari, E., Muscatine, L., Smith, D. C. (1969). Maltose excretion by symbiotic algae of Hydra viridis Proc. R. Soc. Lond. B Biol. Sci. 173, $557-576$.

Chen, M. C., Cheng, Y. M., Sung, P. J., Kuo, C. E., Fanga, L. S. (2003). Molecular identification of Rab7 (ApRab7) in Aiptasia pulchella and its exclusion from phagosomes harboring zooxanthellae. Biochem. Biophys. Res. Commun. 308, $586-$ 595.

Chen, M. C., Cheng, Y. M., Hong, M. C., Fang, L. S. (2004). Molecular cloning of Rab5 (ApRab5) in Aiptasia pulchella and its retention in phagosomes harboring live zooxanthellae, Biochem. Biophys. Res. Commun., 324, 1024 - 1033.

Chen, C. A., Yang, Y. W., Wei, N. V., Tsai, W. S., Fang, L.S. (2005). Symbiont diversity in scleractinian corals from tropical reefs and subtropical non-reef communities in Taiwan. Coral Reefs 24, $11-22$.

Chen, W. N. U., Kang, H. J., Weis, V. M., Mayfield, A. B., Jiang, P. L., Fang, Lo. S., Chen, C. S. (2012). Diel rhythmicity of lipid-body formation in a coralSymbiodinium endosymbiosis. Coral Reefs 31(2), 521 - 534.

Chisholm, S. W. (1981). Temporal patterns of cell division in unicellular algae. In Platt, T. (ed.). Physiological bases of phytoplankton ecology. Bull. Can. J. Fish. Aquatic Sci. 210, 150 - 181. 
References

Coffroth, M. A., Santos, S. R., Goulet, T. L. (2001). Early ontogenetic expression of specificity in a cnidarian-algal symbiosis. Mar. Ecol. Prog. Ser. 222, 85 - 96.

Coffroth, M. A. and Santos, S. R. (2005). Genetic diversity of symbiotic dinoflagellates in the genus Symbiodinium, Protist 156, 19 - 34.

Coffroth, M. A., Poland, D. M., Petrou, E. L., Brazeau, D. A., Holmberg, J. C. (2010). Environmental symbiont acquisition may not be the solution to warming seas for reef-building corals. PLoS ONE 5(10), e13258.doi:10.1371/journal.pone. 0013258.

Coles, S. L. and Brown, B. E. (2003). Coral bleaching-capacity for acclimatization andadaptation. Adv. Mar. Biol. 46, 183 - 223.

Colley, N. J. and Trench, R.K. (1983). Selectivity in phagocytosis and persistence of symbiotic algae by the scyphistoma stage of the jellyfish Cassiopeia xamachana. Proc. R. Soc. Lond. B Biol. Sci. 219, $61-82$.

Colley, N. J. and Trench, R.K. (1985). Cellular events in the reestablishmet of a symbiosis between a marine dinoflagellate and coelenterate. Cell Tissue Res. 239, 93 $-103$.

Collins, H. L., Schaible, U. E., Ernst, J. D., Russell, D. G. (1997). Transfer of phagocytosed particles to the parasitophorous vacuole of Leishmania mexicana is a transient phenomenon preceding the acquisition of annexin I by the phagosome, $J$. Cell Sci. 110, $191-200$.

Cook, C. B. and D'Elia, C. F. (1987). Are natural populations of zooxanthellae ever nutrient-limited? Symbiosis 4, $199-212$.

Cook, C. B. and Davy, S. K. (2001). Are free amino acids responsible for the 'host factor' effects on symbiotic zooxanthellae in extracts of host tissue? Hydrobiologia 461, 71 - 78 . 
References

Crossland, C. J., Barnes, D. J., Borowitzka, M. A. (1980a), Diurnal lipid and mucus production in the staghorn coral Acropora acuminate. Mar. Biol. 60, 81 - 90.

Crossland, C. J., Barnes, D. J., Devereux, M. (1980b). Compartmentation and turnover of organic carbon in the staghorn coral Acropora formosa. Mar. Biol. 59, $181-187$.

Davies, P. S. (1977). Carbon budgets and vertical zonation of atlantic reef corals. In Taylor, D. L. (ed.). Proc. $3^{\text {rd }}$ Intern. Coral Reef Symp. pp. 391 - 396. University of Miami, Rosenstiel School of Marine and Atmospheric Science, Miami, Florida.

Davies, P. S. (1984). The role of zooxanthellae in the nutritional energy requirements of Pocillopora eydouxi. Coral Reefs 2(4), $181-186$.

Davies P. S. (1991). Effect of daylight variations on the energy budgets of shallowwater corals. Mar. Biol. 108, 137 - 144.

Davis, R. H. (2004). The age of model organisms, Nat. Rev. Genet. 5, 69 - 76.

Davy, S. K., Lucas, I. A. N. and Turner, J. R. (1996). Carbon budgets in temperate anthozoan-dinoflagellate symbiosis. Mar. Biol. 126, 773 - 783.

Davy, S. K., Lucas, I. A. N., Turner, J. R. (1997). Uptake and persistence of homologous and heterologous zooxanthellae in the temperate sea anemone Cereus pedunculatus (Pennant). Biol. Bull. 192, 208 - 216.

Davy, S. K and Cook, C. B. (2001a). The influence of "host release factor" on carbon release by zooxanthellae isolated from fed and starved Aiptasia pallida (Verrill). Comp. Biochem. Physiol. A Mol. Integr. Physiol. 129, 487 - 494. 
Davy, S. K and Cook, C. B. (2001b). The relationship between nutritional status and carbon flux in the zooxanthellate sea anemone Aiptasia pallida. Mar. Biol. 139, 999 -1005 .

Davy, S. K., Allemand, D., Weis, V. M. (2012). Cell biology of cnidariandinoflagellate symbiosis, Microbiol. Mol. Biol. R. 76 (2), 1 - 33.

Day, R. J. (1994). Algal symbiosis in Bunodeopsis: sea anemones with auxiliary structures. Biol. Bull. 186, 182 - 194.

de Bary, A. (1879). Die erscheinung der symbiose. In: Trubner, K. J. (ed.) Vortrag auf der versamlung der naturfoscher und artze zu cassel. pp. 1 - 30, Strassburg.

deBoer, M. L., Krupp, D. A., Weis, V. M. (2007). Proteomic and transcriptional analyses of coral larvae newly engaged in symbiosis with dinoflagellates, Comp. Biochem. Physiol. D Gen. Proteom. 2, 63 - 73.

Diekmann, O., Olsen, J., Stam, W., Bak, R. (2003). Genetic variation within Symbiodinium clade B from the coral genus Madracis in the Caribbean (Netherlands Antilles), Coral Reefs 22, 29 - 33.

Dimijian, G. G. (2000). Evolving together: the biology of symbiosis, part 1. Proc. Bayl. Univ. Med. Cent. 13(3), $217-226$.

Dimond, J. and Carrington, E. (2008). Symbiosis regulation in a facultatively symbiotic temperate coral: zooxanthellae division and expulsion. Coral Reefs 27, $601-604$.

Domotor, S. L. and D'Elia, C. F. (1984). Nutrient uptake kinetics and growth of zooxanthellae maintained in laboratory culture. Mar. Biol. 80, 93 - 101.

Domart-Coulon, I. J., Elbert, D. C., Scully, E. P., Calimlim, P. S., Ostrander, G. K. (2001). Aragonite crystallization in primary cell cultures of multicellular isolates 


\section{References}

from a hard coral, Pocillopora damicornis. Proc. Natl. Acad. Sci. USA, 98, 11885 11890.

Douglas, A. E. and Smith, D. C. (1983). The costs of symbionts to the host in green hydra. In Schenk, H.E.A, Schwemmler, W. (ed). Endocytobiology II. pp. 631 - 647, Walter de Gruyter; Berlin.

Douglas, A. E. and Smith, D. C. (1984). The green hydra symbiosis. VIII. Mechanisms in symbiont regulation. Proc. R. Soc. Lond. B Biol. Sci. 221, $291-319$.

Douglas A. E. (1994). Symbiotic interactions. Oxford University Press, Oxford and New York.

Douglas, A. E. (2003). Coral Bleaching - how and why? Mar. Pollut. Bull. 46, 385 392.

Dubinsky, Z. (1990). Coral Reefs. In Dubinsky, Z. (ed.). Ecosystems of the world 25. Elsevier, Amsterdam.

Dunn, S. R., Bythell, J. C., Le Tissier, M. D. A., Burnett, W. J., Thomason, J. C. (2002). Programmed cell death and cell necrosis activity during hyperthermic stressinduced bleaching of the symbiotic sea anemone Aiptasia sp. J. Exp. Mar. Biol. Ecol. $272,29-53$.

Dunn, S. R., Phillips, W. S., Green, D. R., Weis, V. M. (2007). Knockdown of actin and caspase gene expression by RNA interference in the symbiotic anemone Aiptasia pallida, Biol. Bull. 212, $250-258$.

Dunn, S. R. and Weis, V. M. (2009). Apoptosis as a post-phagocytic winnowing mechanism in a coral-dinoflagellate mutualism. Environ. Microbiol. 11, 268 - 276.

Edmunds, P. J. and Davies, P. S. (1986). An energy budget for Porites porites (Scleractinia). Mar. Biol. 92(3), 339 - 347. 
References

Edmunds, P. J. and Davies, P. S. (1989). An energy budget for Porites porites (Scleractinia), growing in a stressed environment, Coral Reefs 8, $37-43$.

Ewald, P. (1983). Host parasite relations, vectors, and the evolution of disease severity. Annu. Rev. Ecol. System, 14, 465 - 485.

Fadlallah, T. H. (1983), Sexual reproduction, development and larval biology in scleractinian corals. A review. Coral Reefs 2, 129 - 150.

Falkowski, P. G., Dubinsky, Z., Muscatine, L., Porter, J. W. (1984). Light and the bioenergetics of a symbiotic coral. Bioscience 34, 705- 709 .

Falkowski, P. G. and LaRoche, J. (1991). Acclimation to spectral irradiance in algae, J. Phycol. 27, $8-14$.

Falkowski, P. G., Dubinsky, Z., Muscatine, L., McCloskey, L. (1993). Populationcontrol in symbiotic corals. Bioscience 43, 606-611.

Fang, L. S., Huang, S. P., Lin, K. L. (1997). High temperature induces the synthesis of heat-shock proteins and the elevation of intracellular calcium in the coral Acropora grandis. Coral Reefs 16, 127 - 131.

Farrant, P. A., Borowitzka, M. A., Hinde, R., King, R. J. (1987a). Nutrition of the temperate Australian soft coral Capnella gaboensis. I. Photosynthesis and carbon fixation. Mar. Biol. 95, 565 - 574.

Farrant P. A., Borowitzka M. A., Hinde R. and King R. J. (1987b). Nutrition of the temperate Australian soft coral Capnella gaboensis.II. The role of zooxanthellae and feeding. Mar. Biol. 95, $575-581$.

Fautin D. G. and Buddemeier R.W. (2004). Adaptive bleaching: a general phenomenon. Hydrobiologia 530/531, 459 - 460. 
Feng, Y., Press, B., Wandinger-Ness, A. (1995). Rab 7: an important regulator of late endocytic membrane traffic, J. Cell Biol., 131, 1435 - 1452.

Fenton-Navarro, B., Arreguin, L. B., Garcia-Hernandez, E., Heimer, E. B., Aguilar, M., Rodriguez, A. C., Arreguin-Espinosa, R. (2003). Purification and structural characterization of lectins from the cnidarian Bunodeopsis antillienis, Toxicon 42, $525-532$.

Ferrari, G., Langen, H., Naito, M. Pieters, J. (1999). A coat protein on phagosomes involved in the intracellular survival of mycobacteria, Cell 97, 435 - 447.

Fine, P. (1975). Vectors and vertical transmission: an epidemiological perspective. Ann. N. Y. Acad. Sci. 266. $173-194$.

Fishman, Y., Zlotkin, E., Sher, D. (2008). Expulsion of symbiotic algae during feeding by the green hydra - a mechanism for regulating symbiont density? PLoS ONE 3, e2603. doi:10.1371/journal.pone.0002603.

Fitt, W. K. and Trench, R. K., (1981). Spawning, development, and acquisition of zooxanthellae by Tridacna squamosa (Mollusca: Bivalvia). Biol. Bull. 161, 213 235.

Fitt W. K. and Trench R. K. (1983). Endocytosis of the symbiotic dinoflagellate Symbiodinium microadriaticum Freudenthal by endodermal cells of the scyphistomae of Cassiopeia xamachana and resistance of the algae to host digestion, J. Cell. Sci. 64, $195-212$.

Fitt, W. K. (1984). The role of chemosensory behavior of Symbiodinium microadriaticum, intermediate hosts, and host behaviour in the infection of coelenterates and molluscs with zooxanthellae. Mar. Biol. 81, 9-17. 
References

Fitt, W. K. (1985). Effect of different strains of the zooxanthella Symbiodinium microadriaticum on growth and survival of their coelenterate and molluscan hosts. Proc. Int. Congr. Coral Reefs 6, $131-136$.

Fitt, W. K. and Cook, C. B. (1990). Some effects of host feeding on growth ofzooxanthellae in the marine hydroid Myrionema ambionense in the laboratoryand in nature. In Narclon P (ed), Endocytobiology IV. pp 281 - 284, INRA, Paris, France.

Fitt, W. K. and Warner, M. E. (1995). Bleaching patterns of four species of Caribbean reef corals. Biol. Bull. 189, 298 - 307.

Fitt, W. K. (2000). Cellular growth of host and symbiont in a cnidarian zooxanthellar symbiosis. Biol. Bull. 198, 110 - 120.

Fitt, W. K., McFarland, F. K., Warner, M. E., Chilcoat, G. C. (2000). Seasonal patterns of tissue biomass and densities of symbiotic dinoflagellates in reef corals and relation to coral bleaching. Limnol. Oceanogr. 45, $677-685$.

Fitt, W. K. and Cook, C. B. (2001). The effects of feeding or addition of dissolved inorganic nutrients in maintaining the symbiosis between dinoflagellates and a tropical marine cnidarian. Mar. Biol. 139, 507 - 517.

FitzGerald, L. M. and Szmant, A. M. (1988). Amino acid metabolism: adaptations to low nutrient conditions? Proc. $6^{\text {th }}$ Int. Coral Reef Symp. 3, 5 - 9.

Forero, M. E., Marin, M., Corrales, A., Llano, I., Moreno, H., Camacho, M. (1999). Leishmania amazonensis infection induces changes in the electrophysiological properties of macrophage-like cells. J. Membr. Biol., 170, 173 - 180.

Freudenthal, H. D. (1962). Symbiodinium gen. nov. and Symbiodinium microadriaticum sp. nov., a zooxanthella: taxonomy, life cycle, and morphology. $J$. Protozool. 9, 45 - 52. 


\section{References}

Friis, R. R. (1972). Interaction of L cells and Chlamydia psittaci: entry of the parasite and host responses to its development, J. Bacteriol. 110, $706-721$.

Furla, P., Bénazet-Tambutté, S., Jaubert, J., Allemand, D. (1998). Functional polarity of the tentacle of the sea anemone Anemonia viridis: role in inorganic carbon acquisition. Am. J. Physiol., 274, R303 - R310.

Furla, P., Allemand, D., Shick, J. M., Ferrier-Pagès, C., Richer, S., Plantivaux, A., Merle, P. L., Tambutte, S. (2005). The symbiotic anthozoan: a physiological chimera between alga and animal. Integr. Comp. Biol. 45, 595 - 604.

Ganot, P., Moya, A., Magnone, V., Allemand, D., Furla, P., Sabourault, C. (2011). Adaptations to endosymbiosis in a cnidarian-dinoflagellate association: differential gene expression and specific gene duplications. PLoS Genet. 7(7), e1002187.doi:10.1371/journal.pgen.1002187.

Gates, R. D. and Muscatine, L. (1992). Three methods for isolating viable anthozoan endoderm cells with their intracellular symbiotic dinoflagellates, Coral Reefs 11, 143 -145 .

Gates, R. D., Baghdasarian, G., Muscatine, L. (1992). Temperature stress causes host cell detachment in symbiotic cnidarians implications for coral bleaching. Biol. Bull. $182,324-332$.

Gates, R. D., Hoegh-Guldberg, O., McFall-Ngai, M. J., Bil, K. Y., Muscatine, L. (1995). Free amino acids exhibit anthozoan - host factor activity: They induce the release of photosynthate from symbiotic dinoflagellates in vitro. Proc. Natl. Acad. Sci. U. S. A. 92, $7430-7434$.

Gautret, P., Cuif, J. P., Freiwald, A. (1997). Composition of soluble mineralizing matrices in azooxanthellate and non-zooxanthellate scleractinian corals: biochemical assessment of photosynthetic metabolism through the study of a skeletal feature. Facies 36, 189 - 194. 
Glynn, P. W. (1991). Coral reef bleaching in the 1980s and possible connections with global warming. Trends Ecol. Evol. 6, 175 - 179.

Glynn, P. W. and D’Croz, L. (1990). Experimental evidence for high temperature stress as the cause of El Niño-coincident coral mortality, Coral Reefs 8, $181-191$.

Goiran, C., Allemand, D., Galgani, I. (1997). Transient $\mathrm{Na}^{+}$stress in symbiotic dinoflagellates after isolation from coral-host cells and subsequent immersion in seawater. Mar. Biol. 129, 581 - 589.

Gómez-Cabrera, M. C., Ortiz, J. C., Loh, W. K. W., Ward, S., Hoegh-Guldberg, O. (2008). Acquisition of symbiotic dinoflagellates (Symbiodinium) by juveniles of the coral Acropora longicyathus. Coral Reefs 27, 219 - 226.

Goulet, T. L., Cook, C. B., Goulet, D. (2005). Effects of short-term exposure to elevated temperatures and light levels on photosynthesis of different host-symbiont combinations in the Aiptasia pallida/Symbiodinium symbiosis. Limnol. Oceanogr. 50, $1490-1498$.

Goulet, T. L. (2006). Most corals may not change their symbionts. Mar. Ecol. Prog. Ser. 321, $1-7$.

Grant, A. J., Rémond, M., People, J., Hinde, R. (1997). Effects of host-tissue homogenate of the scleractinian coral Plesiastrea versipora on glycerol metabolism in isolated symbiotic dinoflagellates. Mar. Biol. 128, $665-670$.

Grant, A. J., Rémond, M., Hinde, R. (1998). Low molecular-weight factor from Plesiastrea versipora (Scleractinia) that modifies release and glycerol metabolism of isolated symbiotic algae. Mar. Biol. 130, 553 - 557.

Grant, A. J., Rémond, M., Withers, K. J. T., Hinde, R. (2001). Inhibition of algal photosynthesis by a symbiotic coral. Hydrobiologia 461, $63-69$. 


\section{References}

Grant, A. J., Trautman, D. A., Frankland, S., Hinde, R. (2003). A symbiosome membrane is not required for the actions of two host signalling compounds regulating photosynthesis in symbiotic algae isolated from cnidarians. Comp. Biochem. Physiol. A Mol. Integr. Physiol. 135, 337 - 345.

Grant, A. J., Rémond, M., Starke-Peterkovic, T., Hinde, R. (2006a). A cell signal from the coral Plesiastrea versipora reduces starch synthesis in its symbiotic alga, Symbiodinium sp. Comp. Biochem. Physiol. A Mol. Integr. Physiol. 144, 458 - 463.

Grant, A. J., Trautman, D. A., Menz, I., Hinde, R. (2006b). Separation of two cell signalling molecules from a symbiotic sponge that modify algal carbon metabolism. Biochem. Biophys. Res. Commun. 348, 92 - 98.

Grottoli, A. G., Rodrigues, L. J., Palardy, J. E. (2006). Heterotrophic plasticity and resilience in bleached corals. Nature 440, $1186-1189$.

Hall, B. F., Furtado, G. C., Joiner, K. A. (1991). Characterization of host cell-derived membrane proteins of the vacuole surrounding different intracellular forms of Trypanosoma cruzi in J774 cells, J. Immunol, 147, 4313 - 4321.

Harland, A. D., Fixter, L. M., Davies, P. S., Anderson, R. A. (1991). Distribution of lipids between the zooxanthellae and animal compartment in the symbiotic sea anemone Anemonia viridis: wax esters, triglycerides and fatty acids. Mar. Biol. 110, $13-19$.

Harland, A. D. and Davies, P. S. (1995). Symbiont photosynthesis increases both respiration and photosynthesis in the symbiotic sea anemone Anemonia viridis. Mar. Biol. 123(4), 715 - 722 .

Helman, Y., Natale, F., Sherrell, R. M., Lavigne, M., Starovoytov, V., Gorbunov, M. Y., Falkowski, P. G. (2008). Extracellular matrix production and calcium carbonate precipitation by coral cells in vitro, Proc. Natl. Acad. Sci. U. S. A. 105, $54-58$. 
References

Hennige, S. J., Suggett, D. J., Warner, M. E., Mc Dougall, K. E., Smith, D. J. (2009). Photophysiology of Symbiodinium revisited: bio-physical and bio-optical signatures, Coral Reefs 28, $179-195$.

Herrera, F. C., Lopez, I., Egea, R., Zanders, I. P. (1989). Short term osmotic responses of cells and tissues of the sea anemone, Condylactis gigantea. Comp. Biochem. Physiol. A Mol. Integr. Physiol. 92, 377 - 384.

Hinde R. (1988). Factors produced by symbiotic marine invertebrates which affect translocation between the symbionts. In Scannerini, S., Smith, D. C., BonfanteFasolo, P., Gianninazzi-Pearson, V. (ed). Cell-to-cell signals in plant, animal and microbial symbiosis. pp. 311 - 324, Springer-Verlag, Berlin, Germany.

Hoegh-Guldberg, O., McCloskey, L. R., Muscatine, L. (1987). Expulsion ofzooxanthellae by symbiotic cnidarians from the Red Sea. Coral Reefs 5, $201-204$.

Hoegh-Guldberg, O. and Smith, G. J. (1989). The effect of sudden changes in temperature, light and salinity on the population density and export of zooxanthellae from the reef corals Stylophora pistillata (Esper) and Seriatopora hystrix (Dana). J. Exp. Mar. Biol. Ecol. 129, 279 - 303.

Hoegh-Guldberg, O., Jones, R, J., Ward, S., Loh, W. K. (2002). Is coral bleaching really adaptive? Nature $\mathbf{4 1 5}, 601-602$.

Hoegh-Guldberg, O., Mumby, P. J., Hooten, A. J., Steneck, R. S., Greenfield, P., Gomez, E., Harvell, C. D., Sale, P. F., Edwards, A. J., Caldeira, K., Knowlton, N., Eakin, C. M., Iglesias Prieto, R., Muthiga, N., Bradbury, R. H., Dubi, A., Hatziolos, M. E. (2007). Coral reefs under rapid climate change and ocean acidification. Science 318, $1737-1742$.

Hoegh-Guldberg, O. and Bruno, J. F. (2010). The impact of climate change on the world's marine ecosystems. Science 328, 1523 - 1528. 


\section{References}

Hohman, T. C., McNeil, P. L., Muscatine, L. (1982). Phagosome-lysosome fusion inhibited by algal symbionts of Hydra viridis, J. Cell Biol. 94, 56 - 63.

Hoogenboom, M., Beraud, E., Ferrier-Pagès, C. (2010). Relationship between symbiont density and photosynthetic carbon acquisition in the temperate coral Cladocora caespitosa. Coral Reefs 29, 21 - 29.

Hong, M. C., Huang, Y. S., Lin, W. W., Fang, L. S., Chen, M. C. (2009). ApRab3, a biosynthetic Rab protein, accumulates on the maturing phagosomes and symbiosomes in the tropical sea anemone, Aiptasia pulchella. Comp. Biochem. Physiol. B Biochem. Mol. Biol. 152(3), 249 - 259.

Horwitz, M. A. (1983). The Legionnaire's disease bacterium (Legionella pneumophila) inhibits phagosome-lysosome fusion in human monocytes, J. Exp. Med. 158, 2108 - 2126.

Houlbrèque, F. and Ferrier-Pagès, C. (2008). Heterotrophy in tropical scleractinian corals. Biol. Rev. 84, $1-17$.

Hrdlicka L. A., (2000). The effects of symbiosis on glycerol metabolism in the sea anemone Aiptasia pallida. Masters thesis, Florida Institute of Technology, Melbourne, Florida.

Hunter, T. (1984). The energetics of asexual reproduction: Pedal laceration in the symbiotic sea anemone Aiptasia pulchella. J. Exp. Mar. Biol. Ecol. 83, 127 - 147.

Hunter, C. L., Morden, C. W., Smith, C. M. (1997). The utility of ITS sequences in assessing relationships among zooxanthellae and corals, Proc. $8^{\text {th }}$ Int. Coral Reef Sym. 2, $1599-1602$.

Iglesias-Prieto, R., Matta, J. L., Robins, W. A., Trench, R. K. (1992). Photosynthetic response to elevated temperature in the symbiotic dinoflagellate Symbiodinium microadriaticum in culture. Proc. Natl. Acad. Sci. 89, $302-305$. 
References

Iglesias-Prieto, R. and Trench, R. K. (1997). Acclimation and adaptation to irradiance in symbiotic dinoflagellates. II. Response of chlorophyll-protein complexes to different photon-flux densities. Mar. Biol. 130, 23 - 33.

Ishikura, M., Adachi, K., Maruyama, T. (1999). Zooxanthellae release glucose in the tissue of a giant clam, Tridacna crocea. Mar. Biol. 133, $665-673$.

Jeon, K. W. and Jeon, M. S. (1976). Endosymbiosis in amoebae: Recently established endosymbionts have become required cytoplasmic components, J. Cell. Physiol. 89(2), 337 - 344.

Jimbo, M., Yanohara, T., Koike, K., Sakai, R., Muramoto, K., Kamiya, H. (2000). The D-galactose-binding lectin of the octocoral Sinularia lochmodes: characterization and possible relationship to the symbiotic dinoflagellates, Comp. Biochem. Physiol. B Biochem. Mol. Biol. 125, 227 - 236.

Jimbo, M., Koike, K., Sakai, R., Muramoto, K., Kamiya, H. (2005). Cloning and characterization of a lectin from the octocoral Sinularia lochmodes, Biochem. Biophys. Res. Commun. 330, $157-162$.

Jolley, E. and Smith, D. C. (1980), The green hydra symbiosis II. The biology of the establishment of the association. Proc. R. Soc. Lond. B. Biol. Sci. 207, $311-333$.

Jones, T. C., Yeh, S., Hirsch. J. G. (1972). The interaction between Toxoplasma gondii and mammalian cells. I. Mechanism of entry and intracellular fate of the parasite. J. Exp. Med. 136, $1157-1172$.

Jones, R. J. and Yellowlees, D. (1997). Regulation and control of intracellular algae (equals zooxanthellae) in hard corals. Proc. R. Soc. Lond. B. Biol Sci. 352, 457 - 468. 


\section{References}

Jones, R. J., Hoegh-Guldberg, O., Larkum, A. W. D., Schreiber, U. (1998). Temperature-induced bleaching of corals begins with impairment of the $\mathrm{CO}_{2}$ fixation mechanism in zooxanthellae. Plant Cell Environ. 21, 1219 - 1230.

Jones, A. M., Berkelmans, R., van Oppen, M. J. H., Mieog, J.C., Sinclair, W. (2008), A community change in the algal endosymbionts of a scleractinian coral following a natural bleaching event: Field evidence of acclimatization. Proc. R. Soc. Lond. B Biol. Sci, 275, $1359-1365$.

Kazandjian A., Shepherd, V. A., Rodriguez-Lanetty, M., Nordemeier, W., Larkum, A. W. D., Qunnell, R. G. (2008). Isolation of symbiosomes and the symbiosome membrane complex from the zoanthid Zoanthus robustus. Phycologia 47, $294-306$.

Kellogg, R. B. and Patton, J. S. (1983). Lipid droplets, medium of energy exchange in the symbiotic anemone Condylactis gigantean - a model coral polyp. Mar. Biol. 75, $137-149$.

Kevin, M. J., Hall, W. T., McLaughlin, J. J. A., Zahl, P. A. (1969). Symbiodinium microadriaticum Freudenthal, a revised taxonomic description, ultrastructure, $J$. Phycol. 5, $341-350$.

Kim, K. J., Na, Y. E., Jeon, K. W. (1994). Bacterial endosymbiont-derived lipopolysaccharides and a protein on symbiosome membranes in newly infected amoebae and their roles in lysosome- symbiosome fusion, Infect. Immun. 62, 65 71.

Kinzie, R. A. (1974). Experimental infection of aposymbiotic gorgonian polyps with zooxanthellae. J. Exp. Mar. Biol. Ecol. 15, 335 - 345.

Kinzie, R. A. and Chee, G. S. (1979). The effect of different zooxanthellae on the growth of experimentally reinfected hosts. Biol. Bull. 156, 315 - 327. 


\section{References}

Kinzie, R. A., Takayama, M., Santos, S. R., Coffroth, M.A. (2001). The adaptive bleaching hypothesis: Experimental tests of critical assumptions, Biol. Bull. 200, 51 58.

Koike, K., Jimbo, M., Sakai, R., Kaeriyama, M., Muramoto, K., Ogata, T., Maruyama, T., Kamiya, H. (2004). Octocoral chemical signalling selects and controls dinoflagellate symbionts, Biol. Bull. 207, $80-86$.

Kuo, J., Chen, M. C., Lin, C. H., Fang, L. S. (2004). Comparative gene expression in the symbiotic and aposymbiotic Aiptasia pulchella by expressed sequence tag analysis, Biochem. Biophys. Res. Commun. 318, $176-186$.

Kvennefors, E. C., Leggat, W., Kerr, C. C., Ainsworth, T. D., Hoegh-Guldberg, O., Barnes, A. C. (2010) Analysis of evolutionarily conserved innate immune components in coral links immunity and symbiosis. Dev. Comp. Immunol. 34, $1219-$ 1229.

LaJeunesse, T. C. (2001). Investigating the biodiversity, ecology, and phylogeny of endosymbiotic dinoflagellates in the genus Symbiodinium using the ITS region: In search of a “species”' level marker. J. Phycol. 37, 866 - 880.

LaJeunesse, T. C. (2002). Diversity and community structure of symbiotic dinoflaggellates from Caribbean coral reefs. Mar. Biol. 141, 387 - 400.

LaJeunesse, T. C., Loh, W. K. W., van Woesik, R., Hoegh-Guldberg, O., Schmidt, G, W., Fitt, W. K. (2003). Low symbiont diversity in southern Great Barrier Reef corals, relative to those of the Caribbean. Limnol. Oceanogr. 48(5), 2046 - 2054.

LaJeunesse, T. C., Thornhill, D. J., Cox, E. F., Stanton, F. G., Fitt, W. K., Schmidt, G. W. (2004a). High diversity and host specificity observed among symbiotic dinoflagellates in reef coral communities from Hawaii, Coral Reefs 23, 596 - 603. 
LaJeunesse, T. C., Bhagooli, R., Hidaka, M., Done, T., deVantier, L., Schmidt, G. W., Fitt, W. K., Hoegh-Guldberg, O. (2004b). Closely-related Symbiodinium spp. differ in relative dominance within coral reef host communities across environmental, latitudinal, and biogeographic gradients, Mar. Ecol. Prog. Ser, 284, $147-161$.

LaJeunesse, T. C. (2005). "Species" radiations of symbiotic dinoflagellates in the Atlantic and Indo-Pacific since the Miocene-Pliocene transition. Mol. Biol. Evol. 22, $570-581$.

Lee, J. J. and Anderson, O. R. (1991). Symbiosis in foraminifera, In Lee, J. J. and Anderson, O. R. (ed.). Biology of Foraminifera, pp. 157 - 220, Academic Press, London.

Leggat, W., Badger, M. R., Yellowlees, D. (1999). Evidence for an inorganic carbonconcentrating mechanism in the symbiotic dinoflagellate Symbiodinium sp. Plant. Physiol. 121, $1247-125$.

Leletkin, V. (2000). The energy budget of coral polyps. Russ. J. Mar. Biol. 26, $389-$ 398

Lerouge, P., Roche, P., Faucher, C., Maillet, F., Truchet, G., Prome, J. C., Denarie, J. (1990). Symbiotic host-specificity of Rhizobium meliloti is determined by a sulphated and acylated glucosamine oligosaccharide signal. Nature 344, 781 - 784.

Lesser, M. P. and Shick, J. M. (1990). Effects of visible and ultraviolet radiation on the ultrastructure of zooxanthellae (Symbiodinium sp.) in culture and in situ. Cell Tissue Res. 261, $501-508$.

Lesser, M. P., Stochaj, W. R., Tapley, D. W., Shick, J. M. (1990). Bleaching in coral reef anthozoans: Effects of irradiance, ultraviolet radiation and temperature on the activities of protective enzymes against active oxygen, Coral Reefs 8, $225-232$. 


\section{References}

Lesser, M. P. (1996). Elevated temperatures and ultraviolet radiation cause oxidativestress and inhibit photosynthesis in symbiotic dinoflagellates. Limnol. Oceanogr. 41, $271-283$.

Lesser, M. P. (2004). Experimental biology of coral reef ecosystems. J. Exp. Mar. Biol. Ecol. 300, $217-252$.

Lesser, M. P. and Farrell, J. H. (2004). Exposure to solar radiation increases damage to both host tissues and algal symbionts of corals during thermal stress, Coral Reefs 23, $367-377$.

Lesser, M. P. (2006). Oxidative stress in marine environments: biochemistry and physiological ecology. Annu. Rev. Physiol. 68, 253 - 278.

Lewis, J. B. and Price, W. S. (1975). Feeding mechanisms and feeding strategies of Atlantic reef corals. J. Zool. Lond. 176, $527-544$.

Lewis, D. H. and Smith, D. C. (1971). Autotrophic nutrition of symbiotic marine coelenterates with special reference to hermatypic corals. I. Movement of photosynthetic products between the symbionts. Proc. R. Soc. Lond. B Biol. Sci. 178, $111-129$.

Lewis, C. L. and Coffroth, M. A. (2004). The acquisition of exogenous algal symbionts by an octocoral after bleaching. Science 304, 1490 - 1492.

Lin, K. L., Wang, J. T., Fang, L. S. (2000). Participation of glycoproteins in zooxanthella cell walls in the establishment of a symbiotic relationship with the sea anemone, Aiptasia pulchella. Zool. Stud. 39, $172-178$.

Linden, O., Souter, D., Wilhelmsson, D., Obura, D. (2002). Coral reef degradation in the Indian Ocean, 278p CORDIO, East Africa. 


\section{References}

Little, A. F., van Oppen, M. J. H., Willis, B. L. (2004). Flexibility in algal endosymbioses shapes growth in reef corals. Science 304, $1492-1494$.

Logan, D. K., LaFlamme, A. C., Weis, V. M., Davy, S. K. (2010). Flow cytometric characterization of the cell surface glycans of symbiotic dinoflagellates (Symbiodinium spp.). J. Phycol. 46, 525 - 533.

Loram, J. E., Trapido-Rosenthal, H. G., Douglas, A. E. (2007). Functional significance of genetically different symbiotic algae Symbiodinium in a coral reef symbiosis. Mol. Ecol. 16, 4849 - 4857.

Luo, Y. J., Wang L. H., Chen, W. N. U., Peng, S. E., Tzen, J. T. C., Hsiao, Y. Y., Fang, L. S., Chen, C. S. (2009). Ratiometric imaging of gastrodermal lipid bodies in coral-dinoflagellate endosymbiosis. Coral Reefs 28, 289 - 301.

MacIntyre, H. L., Kana, T. M., Anning, T., Geider. R. J. (2002). Photoacclimation of photosynthesis irradiance response curves and photosynthetic pigments in microalgae and cyanobacteria, J. Phycol. 38, $17-38$.

Markell, D. A., Trench, R. K., Iglesias-Prieto, R. (1992). Macromolecules associated with the cell-walls of symbiotic dinoflagellates, Symbiosis 12, $19-31$.

Markell, D. A. and Wood-Charlson, E. (2010). Immunocytochemical evidence that symbiotic algae secrete potential recognition signal molecules in hospite. Mar. Biol. $157,1105-1111$.

McAuley, P. J. (1981), Control of cell division of the intracellular Chlorella symbionts in green hydra. J. Cell Sci. 47, $197-206$.

McAuley, P. J. (1982). Temporal relationships of host cell and algal mitosis in the green hydra symbiosis. J. Cell Sci. 58, $423-431$. 
References

McAuley, P. J. and Smith. D. C. (1982). The green hydra symbiosis. VII. Conservation of the host cell habitat by the symbiotic algae. Proc. R. Soc. Lond. B Biol. Sci. 216, $415-426$.

McAuley, P. J. (1985a). The cell cycle of symbiotic Chlorella. I. The relationship between host feeding and algal cell growth and division. J. Cell Sci. 77, 225 - 239.

McAuley, P. J. (1985b). The cell cycle of symbiotic Chlorella. II. The effect of continuous darkness. J. Cell Sci. 77, $241-253$.

McAuley, P. J. (1985c). Regulation of numbers of symbiotic Chlorella indigestive cells of green hydra. Endocytobiosis Cell. Res. 2, 179 - 190.

McAuley, P. J. and Muscatine L. (1986). The cell cycle of symbiotic Chlorella. IV. DNA content of algae slowly increases during host starvation of green hydra. J. Cell Sci. 85, $73-84$.

McAuley, P. J. and Darrah, P. R. (1990). Regulation of numbers of symbiotic Chlorella by density-dependent division. Philos. Trans. R. Soc. Lond. B Biol. Sci. 329, $55-63$.

McCloskey, L. R. and Muscatine, L. (1984). Production and respirations in the Red Sea coral Stylophora pistillata as a function of depth. Proc. R. Soc. B Biol. Sci. 222(1227), $215-230$.

McCloskey, L. R., Muscatine, L., Wilkerson, F. P. (1994). Daily photosynthesis, respiration, and carbon budgets in a tropical marine jellyfish (Mastigias sp.). Mar. Biol. 119, $13-22$.

McCloskey, L. R., Cove, T. G., Verde, E. A. (1996). Symbiont expulsion fromthe anemone Anthopleura elegantissima (Brandt) (Cnidaria; Anthozoa). J. Exp. Mar. Biol. Ecol. 195, 173 - 186. 


\section{References}

McLaughlin, J. J. A. and Zahl, P. A. (1966). Endozoic algae. In Henry, S. M. (ed.) Symbiosis I. pp. 257 - 297, Academic Press, New York.

Meints, R. H. and Pardy, R. L. (1980). Quantitative demonstration of cell surfacement involvement in a plant-animal symbiosis: Lectin inhibition of reassociation. J. Cell. Sci. 43, $239-251$.

Mews, L. K. (1980). The green hydra symbiosis. III. The biotrophic transportof carbohydrate from alga to animal. Proc. R. Soc. Lond. B Biol. Sci. 209, 377 - 401.

Mieog, J. C., van Oppen, M. J. H., Cantin, N. E., Stam, W. T., Olsen, J. L. (2007). Real-time PCR reveals a high incidence of Symbiodiniumclade D at low levels in four scleractinian corals across the Great Barrier Reef: implications for symbiont shuffling. Coral Reefs 26, $449-457$.

Miller, D. J. and Yellowlees, D. (1989). Inorganic nitrogen uptake by symbiotic marine cnidarians: A critical review. Proc. R. Soc. Lond. B Biol. Sci. 237, $109-125$.

Mise, T. and Hidaka, M. (2003). Degradation of zooxanthellae in the coral Acropora nasuta during bleaching. Galaxea J. C. R. S. 5, $33-39$.

Mohrmann, K. and van der Sluijs, P. (1999). Regulation of membrane transport through the endocytic pathway by rab GTPases, Mol. Membr. Biol. 16, $81-87$.

Molea, T. and Munro, P. (1994). Influence of symbiont strain on early growth of tridacnids. Asian Fish. Sci. 7, $91-102$.

Moya, A., Ferrier-Pagès, C., Furla, P., Richier, S., Tambutté, E., Allemand, D., Tambutté, S. (2008). Calcification and associated physiological parameters during a stress event in the scleractinian coral Stylophora pistillata. Comp. Biochem. Physiol. A Mol. Integr. Physiol. 151, 29 - 36. 
References

Mukhopadhyay, A., Funato, K., Stahl, P. D. (1997). Rab7 regulates transport from early to late endocytic compartments in Xenopus oocytes, J. Biol. Chem. 272, 13055 -13059 .

Muller-Parker, G. and Davy, S. K. (2001). Temperate and tropical algal-sea anemone symbioses. Invertebr. Biol. 120, $104-123$.

Muscatine, L. and Hand, C. (1958). Direct evidence for the transfer of materials from symbiotic algae to the tissues of a coelenterate. Proc. Natl. Acad. Sci. U. S. A, 44(12), $1259-1263$.

Muscatine, L. (1967). Glycerol extraction by symbiotic algae from corals and Tridacna and its control by the host. Science 156, 516 - 519.

Muscatine, L., Karakashian, S. J., Karakashian, M. W. (1967). Soluble extracellular products of algae symbiotic with a ciliate, a sponge and a mutant Hydra. Comp. Biochem. Physiol. 20, $1-6$.

Muscatine, L and Cernichiari, E. (1969). Assimilation of photosynthetic products of zooxanthellae by a reef coral. Biol. Bull. 137, $506-523$.

Muscatine, L, Cernichiari, E., Pool, R. R. (1972). Some factors influencing selective release of soluble organic material by zooxanthellae from reef corals. Mar. Biol. 13, $298-308$.

Muscatine, L., Cook, C. B., Pardy, R. L., Pool, R. R. (1975). Uptake, recognition, and maintenance of symbiotic Chlorella by Hydra viridis. Symp. Soc. Exp. Biol. 29, $175-203$.

Muscatine, L. and Porter, J. W. (1977). Reef corals: Mutualistic symbioses adapted to nutrient poor environments. BioScience 27(7), $454-460$. 


\section{References}

Muscatine, L. and Pool, R. R. (1979). Regulation of numbers of intracellular algae. Proc. R. Soc. Lond. B Biol. Sci. 204, $131-139$.

Muscatine, L., McCloskey, L. R., Marian, R. E. (1981). Estimating the daily contribution of carbon from zooxanthellae to coral animal respiration. Limnol.Oceanogr. 26, $601-611$.

Muscatine, L., Falkowski, P. G. and Dubinsky, Z. (1983). Carbon budgets in symbiotic associations. In Schenk, H. E. A. and Schwemmler, W. (ed) Endocytobiology II. Intracellular space as oligogenetic ecosystem. pp 649 - 658. Walter de Gruyter, Berlin and New York.

Muscatine, L, Falkowski, P. G., Porter, J. W., Dubinsky, Z. (1984). Fate of photosynthetic fixed carbon in light-adapted and shade-adapted colonies of the symbiotic coral Stylophora pistillata. Proc. R. Soc. Lond. B Biol. Sci. 222, $181-202$.

Muscatine, L. (1990). The role of symbiotic algae in carbon and energy flux in reef corals.In Dubinsky, Z. (ed.). Ecosystems of the world: Coral reefs. pp. 75 - 87. Elsevier, Amsterdam.

Muscatine, L., Grossman, D., Doino, J. (1991). Release of symbiotic algae by tropical sea anemones and corals after cold shock. Mar. Ecol. Prog. Ser. 77, 233 243.

Muscatine, L., Ferrier-Pagès, C., Blackburn, A., Gates, R. D., Baghdasarian, G., Allemand, D. (1998). Cell-specific density of symbiotic dinoflagellates in tropical anthozoans. Coral Reefs 17, $329-337$.

Neckelmann, N. and Muscatine, L. (1983). Regulatory mechanisms maintainingthe Hydra-Chlorella symbiosis. Proc. R. Soc. Lond. B Biol. Sci. 219, $193-210$.

Obura, D. O. (2005). Resilience and climate change: lessons from coral reefs and bleaching in the western Indian Ocean. CORDIO East Africa. 
Palincsar, J. S., Jones, W. R., Palincsar. E. E. (1988). Effects of isolation of the endosymbiont Symbiodinium microadriaticum (Dinophyceae) from its host Aiptasia pallida (Anthozoa) on cell wall ultrastructure and mitotic rates. Trans. Am. Microsc. Soc. 107, $53-66$.

Papini, E., Satin, B., Bucci, C., de Bernard, M., Telford, J. L., Manetti, R., Rappuoli, R., Zerial, M., Montecucco, C. (1997). The small GTP binding protein rab7 is essential for cellular vacuolation induced by Helicobacter pylori cytotoxin, EMBO 16, $15-24$.

Pardy, R. L. (1981). Cell size distribution of green symbionts from Hydra viridis. Cytobios. 32, $71-77$.

Patton, J. S., Abraham, S., Benson, A. A. (1977). Lipogenesis in the intact coral Pocillopora capitata and its isolated zooxanthellae: Evidence for a light-driven carbon cycle between symbiont and host. Mar. Biol. 44, 235 - 247.

Patton, J. S. and Burris, J. E. (1983). Lipid synthesis and extrusion by freshly isolated zooxanthellae (symbiotic algae). Mar. Biol. 75, 131 - 136.

Patton, J. S., Battey, J. F., Rigler, M. W., Porter, J. W., Black, C. C., Burris, J. F. (1983). A comparison of the metabolism of bicarbonate ${ }^{14} \mathrm{C}$ and acetate $1-{ }^{14} \mathrm{C}$ and the variability of species lipid compositions in reef corals. Mar. Biol. 75, $121-130$.

Peng, S. E., Wang, Y. B., Wang, L. H., Chen, W. N. U, Lu, C. Y., Fang, L. S., Chen, C. S. (2010), Proteomic analysis of symbiosome membranes in Cnidariadinoflagellate endosymbiosis. Proteomics 10, 1002 - 1016.

Peng S. E, Chen, W. N. U., Chen, H. K., Lu, C. Y., Mayfiled, A. B., Fang, L., Chen, C. S. (2011). Lipid bodies in coral-dinoflagellate endosymbiosis: proteomic and ultrastructural studies. Proteomics 11, 3540 - 3555. 
References

Perez, S. F., Cook, C. B., Brooks, W. R. (2001). The role of symbiotic dinoflagellates in the temperature-induced bleaching response of the subtropical sea anemone Aiptasia pallida. J. Exp. Mar. Biol. Ecol. 256, 1 - 14.

Perez, S. F. and Weis, V. M. (2006). Nitric oxide and cnidarian bleaching: An eviction notice mediates the breakdown of the symbiosis, J. Exp. Biol. 209, $2804-$ 2810 .

Peterson, C. H. and Lubchenco, J. (1997). On the value of marine ecosystems to society. In: Daily, G.C. (ed.). Nature's services. Societal dependence on natural ecosystems. pp. 177 - 194, Island Press, New York.

Pfeffer, S. R. (2001). Rab GTPases: Specifying and deciphering organelle identity and function, Trends Cell Biol. 11, 487 - 491.

Pochon, X., Pawlowski, J., Zaninetti, L. andRowan, R. (2001). High genetic diversity and relative specificity among Symbiodinium like endosymbiotic dinoflagellates in soritid foraminiferans. Mar. Biol. 139, 1069 - 1078.

Pochon, X., LaJeunesse, T., Pawlowski, J. (2004). Biogeographic partitioning and host specialization among foramniferan dinoflagellate symbionts (Symbiodinium; Dinophyta). Mar. Biol. 146, 17 - 27.

Pochon, X. and Gates, R. D. (2010). A new Symbiodinium clade (Dinophyceae) from soritid foraminifera in Hawai'i. Mol. Phylogenet. Evol. 56, 492 - 497.

Pool, R. R. (1979). The role of algal antigenic determinants in the recognition of potential algal symbionts by cells of Chlorohydra. J. Cell Sci. 35, $367-379$.

Rahat, M. (1991). An ecological approach to hydra-cell colonization by algaealgae/hydra symbioses. Oikos 62, $381-388$. 


\section{References}

Rands, M. L, Lougham, B. C., Douglas, A. E. (1993). The symbiotic interface in an alga-invertebrate symbiosis. Proc. R. Soc. Lond. B Biol. Sci. 253, $161-165$.

Rees, T. A. V. (1986). The green hydra symbiosis and ammonium. I. The role of the host in ammonium assimilation and its possible regulatory significance. Proc. R. Soc. Lond. B Biol. Sci. 229, 299 - 314.

Rees, T. A. V., Fitt, W. K., Baillie, B., Yellowlees, D. (1993). A method for temporal measurement of hemolymph composition in the giant clam symbiosis and its application to glucose and glycerol levels during a diel cycle. Limnol. Oceanogr. 38, $213-217$.

Regensburg-Tuïnk, A. J. G. and Hooykaas, P. J. J. (1993). Transgenic N. glauca plants expressing bacterial virulence gene virF are converted into hosts for nopaline strains of A. tumefaciens, Nature 363, $69-71$.

Reimer, A. A. (1971). Observations on the relationships between several species of tropical zoanthids (Zoanthidae, Coelenterata) and their zooxanthellae. J. Exp. Mar. Biol. Ecol. 7, 207 - 214.

Reynolds, W. S., Schwarz, J. A., Weis, V. M. (2000). Symbiosis-enhanced gene expression in cnidarian-algal associations: cloning and characterization of a cDNA, sym32, encoding a possible cell adhesion protein, Comp. Biochem. Physiol. A Comp. Physiol. 126, 33 - 44 .

Rinkevich, B. (1989). The contribution of photosynthetic products to coral reproduction. Mar. Biol. 101(2), 259 - 263.

Ritchie, R. J., Eltringham, K., Hinde, R. (1993). Glycerol uptake by zooxanthellae of the temperate hard coral, Plesiastrea versipora (Lamarck). Proc. R. Soc. Lond. B Biol. Sci. 253, $189-195$. 


\section{References}

Roberts, J. M., Fixter, L. M., Davies, P. S. (2001). Ammonium metabolism in the symbiotic sea anemone Anemonia viridis. Hydrobiologia 461, 25 - 35.

Robison, J. D. and Warner, M. E. (2006). Differential impacts of photo-acclimation and thermal stress on the photobiology off our different phylotypes of Symbiodinium (Pyrrhophyta). J. Phycol. 42, $568-579$.

Rodriguez-Lanetty, M., Chang, S. J., Song, J. I. (2003). Specificity of two temperate dinoflagellate-anthozoan associations from the north-western Pacific Ocean. Mar. Biol. 143, 1193 - 1199 .

Rodriquez-Lanetty, M., Krupp, D. A., Weis, V. M. (2004). Distinct ITS types of Symbiodinium in Clade C correlate with cnidarian/dinoflagellate specificity during onset of symbiosis, Mar. Ecol., Prog. Ser. 275, 97 - 102.

Rodriguez-Lanetty, M., Wood-Charlson, E., Hollingsworth, L., Krupp, D. A., Weis, V. M. (2006a). Dynamics of infection and localization of dinoflagellate endosymbionts in larvae of the coral Fungia scutaria during the onset of symbiosis. Mar. Biol. 149, 713 - 719.

Rodriguez-Lanetty, M., Wood-Charlson, E. M., Hollingsworth, L. L., Krupp, D. A., Weis, V. A. (2006b). Temporal and spatial infection dynamics indicate recognition events in the early hours of a dinoflagellate/coral symbiosis, Mar. Biol. 149, 713 719.

Roth, E.; Jeon, K.; Stacey, G. (1988). Homology in endosymbiotic systems: The term "symbiosome", In Palacios, R. D., Verma, P. S. (ed.). Molecular genetics of plantmicrobe interactions. pp. 220 - 225, American Phytopathological Society Press, Minnesota.

Rowan, R. and Powers. D. A. (1991). A molecular genetic classification of zooxanthellae and the evolution of animal-algal symbiosis. Science 251, 1348 1351. 
Rowan, R., Knowlton, N., Baker, A. C., Jara, J. (1997). Landscape ecology of algal symbionts creates variation in episodes of coral bleaching. Nature 388, $265-269$.

Rowan, R. (2004). Thermal adaptation in reef coral symbionts. Nature 430, 742.

Russell, D. G., Xu, S., Chakraborty, P. (1992). Intracellular trafficking and the parasitophorous vacuole of Leishmania mexicana-infected macrophages, J. Cell Sci. 103, $1193-1210$.

Russo, P., Pecorella, M. A., Desantis, A., Dale, B. (1989). pH in eggs of the ascidian Ciona intestinalis at fertilisation and activation, J. Exp. Zool. 250, 329 - 332.

Sachs, J. and Wilcox, T. (2006). A shift to parasitism in the jellyfish symbiont Symbiodinium microadriaticum. Proc. R. Soc. London. B Biol. Sci. 273, 425 - 429.

Sam-Yellowe, T. Y. (1992). Molecular factors responsible for host cell recognition and invasion in Plasmodium falciparum. J. Protozool. 39, 181 - 189.

Santos, S. R., Taylor, D. J., Coffroth, M. A. (2001). Genetic comparisons of freshly isolated versus cultured symbiotic dinoflagellates: Implications for extrapolating to the intact symbiosis, J.Phycol. 37, $900-912$.

Sawyer, S. J. and Muscatine, L. (2001). Cellular mechanisms underlying temperature-induced bleaching in the tropical sea anemone Aiptasia pulchella, J. Exp. Biol. 204, 3443 - 3456.

Schmitz, K. and Kremer, B. P. (1977). Carbon fixation and analysis of assimilates in a coral-dinoflagellate symbiosis. Mar. Biol. 42, 305 - 313.

Schoenberg, D. A., and Trench. R. K. (1976). Specificity of symbioses between marine cnidarians and zooxanthellae, In G. O. Mackie (ed.). Coelenterate ecology and behaviour, pp. 423 - 432. Plenum Press, New York. 
References

Schoenberg, D. A. and Trench, R. K. (1980a). Genetic variation in Symbiodinium (=Gymnodinium) microadriaticum Freudenthal, and specificity in its symbiosis with marine invertebrates. II. Morphological variation in Symbiodinium microadriaticum. Proc. R. Soc. Lond. B Biol. Sci. 207, $429-444$.

Schoenberg, D. A. and Trench, R. K. (1980b). Genetic variation in Symbiodinium (=Gymnodinium) microdriaticum Freudenthal and specificity in its symbiosis with marine invertebrates (III) Specificity and infectivity of Symbiodinium microdriaticum. Proc. R. Soc. Lond. B Biol. Sci. 207, 445 - 460.

Schwarz, J. A. and Weis, V. M. (2003). Localization of a symbiosis-related protein, Sym32, in the Anthopleura elegantissima-Symbiodinium muscatinei association, Biol. Bull. 205, $339-350$.

Shen, S. S. and Steinhardt, R. A. (1978). Direct measurement of intracellular pH during metabolic depression of the sea urchin egg, Nature 272, 253 - 254.

Shinzato, C., Shoguchi, E., Kawashima, T., Hamada, M., Hisata, K., Tanaka, M., Fujie, M., Fujiwara, M., Koyanagi, R., Ikuta, T., Fujiyama, A., Miller, D. J., Satoh, N. (2011). Using the Acropora digitifera genome to understand coral responses to environmental change. Nature 476, 320 - 323.

Sibley, L. D. and Krahenbuhl, J. L. (1989). Modification of host cell phagosomes by Toxoplasma gondii involves redistribution of surface proteins and secretion of a 32 kDa protein, Eur. J. Cell Biol. 47, $81-87$.

Silverstein, R., Correa, A. M. S., Baker, A.C. (2012). Specificity is rarely absolute in coral-algal symbiosis: Implications for coral response to climate change. Proc. $R$. Soc. Lond. B. Biol. Sci. 279 (1738), 2609 - 2618.

Smith, G. J. and Muscatine, L. (1986). Carbon budgets and regulation of the population density of symbiotic algae. Endocyt. Cell Res. 3, $213-238$. 
References

Smith, D. C. and Douglas, A. E. (1987). In Smith D. C. and Douglas A. E. (ed.). The biology of symbiosis, Edward Arnold, Ltd, London.

Smith, D. C. (1993). The symbiotic condition. Symbiosis 14, 3 - 15.

Smith, G. J and Muscatine L. (1999). Cell cycle of symbiotic dinoflagellates: variation in $\mathrm{G}(1)$ phase-duration with anemone nutritional status and macronutrient supply in the Aiptasia pulchella-Symbiodinium pulchrorum symbiosis. Mar. Biol. 134, $405-418$.

Smith, D. J., Suggett, D. J., Baker, N. R. (2005). Is photoinhibition of zooxanthellae photosynthesis the primary cause of thermal bleaching in corals? Glob. Change Biol. 11, 1 - 11 .

Stat. M., Carter, D., Hoegh-Guldberg, O. (2006). The evolutionary history of Symbiodinium and scleractinian hosts - Symbiosis, diversity, and the effect of climate change. Perspect. Plant Ecol. Evol. System 8, 23 - 43.

Stat, M., Morris, E., Gates, R. D. (2008). Functional diversity in coral-dinoflagellate symbiosis. Proc. Natl. Acad. Sci. U. S. A. 105, 9256 - 9261.

Stat, M., Loh, W. K. W., LaJeunesse, T. C., Hoegh-Guldberg, O., Carter, D. A. (2009). Stability of coral-endosymbiont associations during and after a thermal stress event in the southern Great Barrier Reef. Coral Reefs 28, $709-713$.

Stat, M., Bird, C. E., Pochon, X., Chasqui, L., Chauka, L. J., Concepcion, G. T., Logan, D., Takabayashi, M., Toonen, R. J., Gates, R. D. (2011), Variation in Symbiodinium ITS2 sequence assemblages among coral colonies. PLoS ONE 6(1), e15854. doi:10.1371/journal.pone.0015854.

Stat, M. and Gates, R. D. (2011). Clade D symbiodinium in scleractinian corals: A "nugget" of hope, a selfish opportunist, an ominous sign, or all of the above? J. Mar. Biol. 2011, 9. doi:10.1155/2011/730715. 
References

Steele, R. D. (1975). Stages in the life history of a symbiotic zooxanthella in pellets extruded by its host Aiptasia tagetes (Duch. and Mich.) (Coelenterata, Anthozoa). Biol. Bull. 149, $590-600$.

Steele, R. D. (1977). The significance of zooxanthella-containing pellets extrudedby sea anemones. Bull. Mar. Sci. 27, $591-594$.

Steele, R. D. and Goreau, N. I. (1977). The breakdown of symbiotic zooxanthellae in the sea anemone Phyllactis (= Oulactis) flosculifera (Actinaria). J. Zool. 181, 421 437.

Steen, R. G. and Muscatine L. (1984). Daily budgets of photosynthetically fixed carbon in symbiotic zoanthids. Biol. Bull. 167, $477-487$.

Steen, R. G. (1986). Evidence for heterotrophy by zooxanthellae in symbiosis with Aiptasia pulchella. Biol. Bull. 170, $267-278$.

Stimson, J. and Kinzie, R. A. (1991). The temporal pattern and rate of release ofzooxanthellae from the reef coral Pocillopora damicornis (Linnaeus) undernitrogen-enrichment and control conditions. J. Exp. Mar. Biol. Ecol. 153, 63 74.

Strathmann, R. R. (1967). Estimating the organic carbon content of phytoplankton from cell volume or plasma volume. Limnol. Oceanogr. 12, 411 - 418.

Streamer.M. McNeil, Y. R., Yellowlees, D. (1993). Photosynthetic carbon dioxide fixation in zooxanthellae. Mar. Biol. 115, 195 - 198.

Sutton, D. C. and Hoegh-Guldberg, O. (1990). Host-zooxanthella interactions in 4 temperate marine invertebrate symbioses: assessment of effect of host extracts on symbionts. Biol. Bull. 178, $175-186$. 


\section{References}

Takabayashi, M., Santos, S., Cook, C. (2004). Mitochondrial DNA phylogeny of the symbiotic dinoflagellates (Symbiodinium, Dinophyta). J. Phycol. 40, 160 - 164.

Takabayashi, M., Adams, L., Pochon, X., Gates. R. D. (2012). Genetic diversity of free-living Symbiodinium in surface water and sediment of Hawaii and Florida, Coral Reefs, 31(1), 157 - 167.

Takahashi, S., Nakamura, T., Sakamizu, M., Woesik, R. V., Yamasaki, H. (2004). Repair machinery of symbiotic photosynthesis as the primary target of heat stress for reef-building corals. Plant Cell Physiol. 45, 251 - 255.

Taylor, D. L. (1971). Ultrastructure of the 'zooxanthella' Endodinium chattonii in situ. J. Mar. Biol. Assoc. U. K. 51, 227 - 234.

Taylor, D. L. (1974). Symbiotic marine algae: taxonomy and biologicalfitness. In Vernberg, W. B. (ed.). Symbiosis in the sea, pp. 245-262, University of South Carolina Press, Columbia, S. C.

Taylor, C. E., Muscatine, L., Jefferson, D. R. (1989). Maintenance and breakdown of the Hydra-Chlorella symbiosis: a computer model. Proc. R. Soc. Lond. B Biol. Sci. 238, $277-289$.

Tchernov, D., Gorbunov, M. Y., de Vargas, C., Narayan Yadav, S., Milligan, A. J., Haggblom, M., Falkowski, P. G. (2004). Membrane lipids of symbiotic algae are diagnostic of sensitivity to thermal bleaching in corals. Proc. Natl. Acad. Sci. U. S. A. 101, $13531-13535$.

Thornhill, D. J., LaJeunesse, T. C., Kemp, D. W., Fitt, W. K., Schmidt, G. W. (2005). Multi-year, seasonal genotypic surveys of coral-algal symbioses reveal prevalent stability or post-bleaching reversion. Mar. Biol. 148, $711-722$. 
References

Thornton, D. C. O. (2009). Effect of low pH on carbohydrate production by a marine planktonic diatom (Chaetoceros muelleri). Research Letters in Ecology e105901, doi:10.1155/2009/105901.

Titlyanov, E. A., Titlyanova, T. V., Leletkin, V. A., Tsukahara, J., van Woesik, R., Yamazato, K. (1996). Degradation of zooxanthellae and regulation of their density in hermatypic corals. Mar. Ecol. Prog. Ser. 139, 167 - 178.

Titlyanov, E. A., Titlyanova, T. V., Kalita, T. L., Yakovleva, I. M. (2004). Rhythmicityin division and degradation of zooxanthellae in the hermatypic coral Stylophora pistillata. Symbiosis 36, $211-224$.

Titlyanov, E. A., Titlyanova, T. V., Yakovleva, I. M., Kalita, T. L. (2006). Rhythmical changes in the division and degradation of symbiotic algae in hermatypiccorals. Russ. J. Mar. Biol. 32, 12 - 19.

Toller, W. W., Rowan, R., Knowlton. N. (2001a). Zooxanthellae of the Montastraea annularis species complex: patterns of distribution of four taxa of Symbiodinium of different reefs and across depths. Biol. Bull. 201, $348-359$.

Toller, W., Rowan, R., Knowlton, N. (2001b). Repopulation of zooxanthellae in the Caribbean corals Montastraea annularis and M. faveolata following experimental and disease associated bleaching. Biol. Bull. 201, 360 - 373.

Tremblay, P., Peirano, A., Ferrier-Pagès, C. (2011). Heterotrophy in the Mediterranean symbiotic coral Cladocora caespitose comparison with the two other scleractinian species. Mar. Ecol. Prog. Ser. 422, 165 - 177.

Tremblay, P., Grover, R., Maguer, J. F., Legendre, L., Ferrier-Pagès, C. (2012). Autotrophic carbon budget in the coral tissue: a new ${ }^{13} \mathrm{C}$-based model of photosynthate translocation. J. Exp. Biol. 215, $1384-1393$. 


\section{References}

Trench, R. K. (1971a). The physiology and biochemistry of zooxanthellae symbiotic with marine coelenterates. I. The assimilation of photosynthetic products of zooxanthellae by two marine coelenterates. Proc. R. Soc. Lond. B Biol. Sci. 177, 225 -235 .

Trench, R. K. (1971b). The physiology and biochemistry of zooxanthellae symbiotic with marine coelenterates. II. Liberation of fixed ${ }^{14} \mathrm{C}$ by zooxanthellae in vitro. Proc. R. Soc. Lond. B Biol. Sci., 177, 237 - 250.

Trench, R. K. (1971c). The physiology and biochemistry of zooxanthellae symbiotic with marine coelenterates. III. The effect of homogenates of host tissues on the excretion of photosynthetic products in vitro by zooxanthellae from two marine coelenterates. Proc. R. Soc. Lond. B Biol. Sci. 177, $251-264$.

Trench, R. K. (1974). Nutritional potentials in Zoanthus sociatus (Coelenterata, Anthozoa). Helgolander Wiss. Meeresunters 26, 174 - 216.

Trench, R. K. (1979). The cell biology of plant-animal symbiosis, Annu. Rev. Plant Physiol. 30, 485 - 531 .

Trench, R. K. (1987). Dinoflagellates in non-parasitic symbiosis. In Taylor, F. J. R. (ed.). Biology of dinoflagellates. pp. 530 - 570. Blackwell, Oxford, London.

Trench, R. K. and Winsor, H. (1987). Symbiosis with dinoflagellates in two pelagic flat worms Amphiscolops sp. and Haplodiscus sp. Symbiosis 3, 1- 22.

Trench, R. K. (1993). Microalgal-invertebrate symbioses: a review, Endocyt. Cell. Res. 9, $135-175$.

Trench, R. K. (1997). Diversity of symbiotic dinoflagellates and the evolution of microalgal-invertebrate symbioses. Proc. $8^{\text {th }}$ Int. Coral Reef Sym. 2, 1275 - 1286. 
References

Tremblay P., Peirano A., Ferrier-Pagès C. (2011). Heterotrophy in the Mediterranean symbiotic coral Cladocora caespitosa: comparison with the two other scleractinian species. Mar. Ecol. Progr. Ser. 422, 165 - 177.

Tripodi, G. and Santisi, S. (1982). A study on the cell covering of Symbiodinium, a symbiote of the octocoral Eunicella, J. Submicrosc. Cytol. 14, 613-620.

Ulstrup, K. E. and van Oppen, M. J. H. (2003). Geographic and habitat partitioning of genetically distinct zooxanthellae (Symbiodinium) in Acropora corals of the Great Barrier Reef. Mol. Ecol. 12, 3477 - 3484.

Udvardi, M. K. and Day, D. A. (1997). Metabolite transport across symbiotic membranes of legume nodules, Annu. Rev. Plant Physiol. Plant Mol. Biol. 48, $493-$ 523.

Van Oppen, M. J. H., Palstra, F. P., Piquet, A. M. T., Miller, D. J. (2001). Patterns of coral dinoflagellate associations in Acropora: significance of local availability and physiology of Symbiodinium strains and host-symbiont selectivity. Proc. R. Soc. London Ser. B Biol. Sci. 268, 1759 - 1767.

Van Oppen, M. J. H., Mieog, J. C., Sánchez, C. A., Fabricius, K. E. (2005). Diversity of algal endosymbionts (zooxanthellae) in tropical octocorals: the roles of geography and host relationships. Mol. Ecol. 14, 2403 - 2417.

Vega, I. A., Damborenea, M. C., Gamarra-Luques, C., Koch, E., Cueto, J. A., CastroVazquez, A. (2006). Facultative and obligate symbioticassociations of Pomacea canaliculata (Caenogastropoda, Ampullariidae). Biocell 30, 367 - 375.

Venn, A. A., Loram, J. E., Douglas, A. E. (2008). Photosynthetic symbioses in animals. J. Exp. Bot. 59, 1069 - 1080. 


\section{References}

Venn, A., Tambutté, E., Lotto, S., Zoccola, D., Allemand, D., Tambutté, S. (2009). Intracellular pH in symbiotic cnidarians.Proc Natl. Acad. Sci. U. S. A. 106, $16574-$ 16579 .

Venn, A., Tambutté, E., Holcomb, M., Allemand, D., Tambutté, S. (2011). Live tissue imaging shows reef corals elevate $\mathrm{pH}$ under their calcifying tissue relative to seawater, PLos ONE 6(5), e20013. doi:10.1371/journal.pone.0020013.

Verde, E. A. and McCloskey, L. R. (1996a). Carbon budget studies of symbiotic cnidarian anemones - Evidence in support of some assumptions. J. Exp. Mar. Biol. Ecol. 195, $161-171$.

Verde, E. A. and McCloskey, L. R. (1996b). Photosynthesis and respiration of two species of algal symbionts in the anemone Anthopleura elegantissima (Cnidaria; Anthozoa). J. Exp. Mar. Biol. Ecol. 195, 187 - 202.

Verde, E. A. and L. R. McCloskey. (2002). A comparative analysis of the photobiology of zooxanthellae and zoochlorellae symbiotic with the temperate clonal anemone Anthopleura elegantissima (Brandt). II. Effect of light intensity. Mar. Biol. $141,225-239$.

Verde, E. A. and McCloskey. L. R. (2007). A comparative analysis of the photobiology of zooxanthellae and zoochlorellae symbiotic with the temperate clonal anemone Anthopleura elegantissima (Brandt). III. Seasonal effects of natural light and temperature on photosynthesis and respiration. Mar. Biol. 152, 775 - 792.

Veron, J. E. N. (2000). Corals of the world, Vol. 1 - 3. Australian Institute of Marine Science and C. R. R. Qld. Pty. Ltd., Australia.

Vitelli, R., Santillo, M., Lattero, D., Chiariello, M., Bifulco, M., Brunii, C. B., Bucci, C. (1997). Role of the small GTPase RAB7 in the late endocytic pathway, J. Biol. Chem. 272, $4391-4397$. 
References

Vollmer, S. V. and Palumbi, S. R. (2002). Hybridization and the evolution of reef coral diversity, Science 296(5575), 2023 - 2025.

Von Holt, C. (1968). Uptake of glycine and release of nucleoside polyphosphates by zooxanthellae. Comp. Biochem. Physiol. 26, 1071 - 1079.

Von Holt, C. and Von Holt, M. (1968). The secretion of organic compounds by zooxanthellae isolated from various types of Zoanthus. Comp. Biochem. Physiol. 24, $83-92$.

Voolstra, C. R., Schwarz, J. A., Schnetzer, J., Sunagawa, S., Desalvo, M. K., Szmant, A., Coffroth, M. A., Medina, M. (2009). The host transcriptome remains unaltered during the establishment of coral-algal symbioses. Mol. Ecol. 18. 1823 - 1833.

Wakefield, T. S., Kempf, S. C., Farmer, M. A. (1998). The contribution of Symbiodinium microadriaticum to the symbiosome membrane in the anthozoan host Aiptasia pallida. Microsc. Microanal. 4, 1182 - 1183.

Wakefield, T. S., Farmer, M. A., Kempf, S. C. (2000). Revised description of the fine structure of in situ "zooxanthellae" genus Symbiodinium, Biol. Bull, 199, 76 84.

Wakefield, T. S. and Kempf S. C. (2001). Development of host and symbiontspecific monoclonal antibodies and confirmation of the origin of the symbiosome membrane in a cnidarian-dinoflagellate symbiosis. Biol. Bull. 200, 127 - 143.

Wang, J. T. and Douglas, A. E. (1998). Nitrogen recycling or nitrogen conservation in an alga-invertebrate symbiosis? J. Exp. Biol. 201, 2445 - 2453.

Wang, J. T. and Douglas, A. E. (1999). Essential amino acid synthesis and nitrogen recycling in an alga-invertebrate symbiosis. Mar. Biol. 135, 219 - 222. 
References

Wang, L. H., Liu, Y. H., Yu, Y. M., Hsiao, Y. Y., Fang, L. S., Chen, C. S. (2008). Cell cycle propagation is driven by light-darkstimulation in a cultured symbiotic dinoflagellate isolated from corals. Coral Reefs 27, $823-835$.

Warner, M. E., Fitt, W. K., Schmidt, G. W. (1999). Damage to photosystem II insymbiotic dinoflagellates: A determinant of coral bleaching. Proc. Natl. Acad. Sci. U.S. A. 96, $8007-8012$.

Weis, V. M. (1993). Effect of dissolved inorganic carbon concentration on the photosynthesis of the symbiotic sea anemone Aiptasia pulchella Carlgren: Role of carbonic anhydrase. J. Exp. Mar. Biol. Ecol. 174(2), 209 - 225.

Weis, V. M. and Levine, R. P. (1996). Differential protein profiles reflect the different lifestyles of symbiotic and aposymbiotic Anthopleura elegantissima, a sea anemone from temperate waters, .J Exp. Biol. 199, 883 - 892.

Weis, V. M., Reynolds, W. S., deBoer, M. D., Krupp, D. A. (2001). Host-symbiont specificity during onset of symbiosis between the dinoflagellates Symbiodinium spp. and planula larvae of the scleractinian coral Fungia scutaria. Coral Reefs 20, 301 308.

Weis, V. M. (2008). Cellular mechanisms of cndiarian bleaching: Stress causes the collapse of symbiosis. J. Exp. Biol. 211, 3059-3066.

Weis, V. M., Davy, S., Hoegh-Guldberg, O., Rodriguez-Lanetty, M., Pringle, J. (2008). Cell biology in model systems as the key to understanding corals. Trends Ecol. Evol. 23, 369 - 376.

Weis, V. M. and Allemand, D. (2009). What determines coral health? Science 324, $1153-1154$. 


\section{References}

Wijgerde, T., Diantari, R., Lewaru, M. W., Verreth, J. A. J., Osinga, R. (2011). Extracoelenteric zooplankton feeding is a key mechanism of nutrient acquisition for the scleractinian coral Galaxea fascicularis. J. Exp. Biol. 214(20), 3351 - 3357.

Wishnick, M., and Lane, M. D. (1969). Inhibition of ribulose diphosphate carboxylase by cyanide. Inactive ternary complex of enzyme, ribulose diphosphate, and cyanide. J. Biol. Chem. 244, 55 - 59.

Withers, K. J. T., Grant, A. J., Hinde, R. (1998). Effects of free amino acids on the isolated symbiotic algae of the coral Plesiastrea versipora (Lamarck): Absence of a host release factor response. Comp. Biochem. Physiol. Part A Mol. Integr. Physiol., $120,599-607$.

Whitehead, L. F. and Douglas, A. E. (2003). Metabolite comparisons and the identity of nutrients translocated from symbiotic algae to an animal host. J. Exp. Biol. 206, $3149-3157$.

Wood-Charlson, E. M., Hollingsworth, L. H., Krupp, D. A., Weis, V. M. (2006). Lectin/glycan interactions play a role in recognition in a coral/dinoflagellate symbiosis. Cell. Microbiol. 8, $1985-1994$.

Wood-Charlson, E. M. and Weis, V. M. (2009). The diversity of C-type lectins inthe genome of a basal metazoan, Nematostella vectensis. Dev. Comp. Immunol. 33, 881 -889 .

Yellowlees, D., Rees, T. A. V., Leggat, W. (2008). Metabolic interactions between algal symbionts and invertebrate hosts. Plant Cell. Environ. 31, 679-694.

Zerial, M. and McBride, H. (2001). Rab proteins as membrane organizers. Nature Rev. Mol. Cell Biol. 2, 107 - 117. 


\section{Appendix 1}

The influence of symbiont diversity on the photophysiological performance of novel cnidarian-dinoflagellate symbioses; complete carbon budget over entire 12 week period after $A$. pulchella infection with different Symbiodinium types

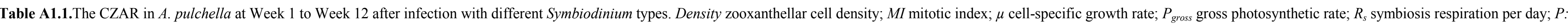

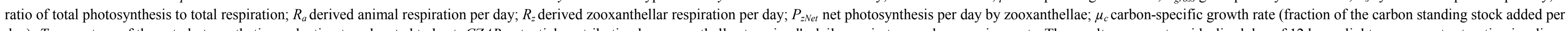

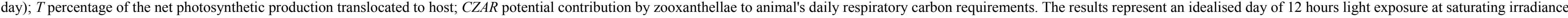
and 12 hours of darkness. Values are means $\pm \mathrm{SE}(\mathrm{n}=4-5$ for all parameters, except algal cell diameter, where $\mathrm{n}=72$ and $M I$, where $\mathrm{n}=32)$.

\begin{tabular}{|c|c|c|c|c|c|c|c|c|c|c|c|c|c|c|c|c|c|c|c|c|c|c|c|c|c|c|c|c|c|c|}
\hline \multirow{2}{*}{$\begin{array}{l}\begin{array}{l}\text { Symbiodinium } \\
\text { type }\end{array} \\
\text { Week }\end{array}$} & \multicolumn{5}{|l|}{ A1.4 } & \multicolumn{5}{|c|}{ Heterologous B1 } & \multicolumn{5}{|l|}{ E2 } & \multicolumn{5}{|l|}{ F5.1 } & \multicolumn{5}{|c|}{ | Homologous (B1) - freshly isolated } & \multicolumn{5}{|c|}{ Homologous (B1) - cultured } \\
\hline & 1 & 2 & 4 & 8 & 12 & 1 & 2 & 4 & 8 & 12 & 1 & 2 & 4 & 8 & 12 & 1 & 2 & 4 & 8 & 12 & & & & & 12 & & & & 8 & \begin{tabular}{|l|}
12 \\
\end{tabular} \\
\hline $\begin{array}{l}\text { Density } \\
\text { (x } 10^{6} \text { cells } \mathrm{mg}^{-1} \\
\text { protein) }\end{array}$ & $\begin{array}{l}0.5 \pm \\
0.07\end{array}$ & $\begin{array}{l}2.1 \pm \\
0.9\end{array}$ & $\begin{array}{l}5.5 \pm \\
2.1\end{array}$ & $\begin{array}{l}3.9 \pm \\
1.0\end{array}$ & $\begin{array}{l}2.9 \pm \\
1.2\end{array}$ & $\begin{array}{l}0.4 \pm \\
0.2\end{array}$ & $\begin{array}{l}2.5 \pm \\
0.9\end{array}$ & $\begin{array}{l}4.0 \pm \\
1.2\end{array}$ & $\begin{array}{l}4.3 \pm \\
0.4\end{array}$ & \begin{tabular}{|l}
$1.3 \pm$ \\
0.06
\end{tabular} & $\begin{array}{l}2.0 \pm \\
0.4\end{array}$ & $\begin{array}{l}2.3 \pm \\
1.0\end{array}$ & $\begin{array}{l}6.1 \pm \\
1.5\end{array}$ & $\begin{array}{l}5.2 \pm \\
0.7\end{array}$ & $\begin{array}{l}4.1 \pm \\
0.2\end{array}$ & $\begin{array}{l}0.4 \pm \\
0.1\end{array}$ & $\begin{array}{l}0.8 \pm \\
0.4\end{array}$ & $\begin{array}{l}4.3 \pm \\
1.2\end{array}$ & $\begin{array}{l}7.3 \pm \\
1.7\end{array}$ & $\begin{array}{l}4.5 \pm \\
2.9\end{array}$ & \begin{tabular}{|l}
$0.3 \pm$ \\
0.05
\end{tabular} & $\begin{array}{l}0.8 \pm \\
0.4\end{array}$ & $\begin{array}{l}3.6 \pm \\
0.4\end{array}$ & $\begin{array}{l}2.4 \pm \\
1.3\end{array}$ & $\begin{array}{l}1.6 \pm \\
0.2\end{array}$ & $\begin{array}{l}0.06 \pm \\
0.02\end{array}$ & $\begin{array}{l}0.2 \pm \\
0.08\end{array}$ & $\begin{array}{l}1.4 \pm \\
0.5\end{array}$ & $\begin{array}{l}1.2 \pm \\
0.5\end{array}$ & $\begin{array}{l}0.5 \pm \\
0.2\end{array}$ \\
\hline $\begin{array}{l}\text { Algal cell } \\
\text { diameter (um) }\end{array}$ & $\begin{array}{l}5.56 \pm \\
032\end{array}$ & $\begin{array}{l}5.88 \pm \\
066\end{array}$ & $\begin{array}{l}9.95 \pm \\
0.55\end{array}$ & \begin{tabular}{|l|}
$10.22 \pm$ \\
015
\end{tabular} & $\begin{array}{l}9.59 \pm \\
0.25\end{array}$ & $\begin{array}{l}5.83 \pm \\
0.17\end{array}$ & $\begin{array}{l}6.19 \pm \\
034\end{array}$ & \begin{tabular}{|l|}
10.63 \\
+028 \\
+028
\end{tabular} & $\begin{array}{l}9.61 \pm \\
0.33\end{array}$ & $\begin{array}{l}9.20 \pm \\
0.15\end{array}$ & $\begin{array}{l}6.88 \pm \\
078\end{array}$ & $\begin{array}{l}6.92 \pm \\
0.53\end{array}$ & $\begin{array}{l}9.03 \pm \\
0.34\end{array}$ & $\begin{array}{l}9.01 \pm \\
0.25\end{array}$ & $\begin{array}{l}8.59 \pm \\
030\end{array}$ & $\begin{array}{l}4.97 \pm \\
023\end{array}$ & $\begin{array}{l}5.23 \pm \\
015\end{array}$ & $\begin{array}{l}8.49 \pm \\
111\end{array}$ & $\begin{array}{l}7.79 \pm \\
0.78\end{array}$ & $\begin{array}{l}7.72 \pm \\
0.08\end{array}$ & \begin{tabular}{|l|}
$6.51 \pm$ \\
019
\end{tabular} & $\begin{array}{l}8.95 \pm \\
0.50\end{array}$ & $\begin{array}{l}8.92 \pm \\
042 \pm\end{array}$ & $\begin{array}{l}9.74 \pm \\
015\end{array}$ & $\begin{array}{l}8.67 \pm \\
0.07\end{array}$ & $\begin{array}{l}8.68 \pm \\
035\end{array}$ & $\begin{array}{l}7.57 \pm \\
012\end{array}$ & $\begin{array}{l}9.32 \pm \\
017\end{array}$ & $\begin{array}{l}8.64 \pm \\
036\end{array}$ & $\begin{array}{l}8.29 \pm \\
0.09\end{array}$ \\
\hline $\begin{array}{l}\text { Derived algal } \\
\text { carbon }(\mathrm{pg} \mathrm{C} \\
\text { cell(-1) }\end{array}$ & $\begin{array}{l}19.72 \\
\pm 3.21\end{array}$ & $\begin{array}{l}24.78 \\
\pm 8.67\end{array}$ & $\begin{array}{l}81.85 \\
\pm \\
12.00\end{array}$ & \begin{tabular}{|l|}
88.47 \\
\pm 2.67
\end{tabular} & $\begin{array}{l}73.14 \\
\pm 3.53\end{array}$ & $\begin{array}{l}21.79 \\
\pm 2.10\end{array}$ & $\begin{array}{l}28.18 \\
\pm 3.95\end{array}$ & $\begin{array}{l}97.98 \\
\pm 5.85\end{array}$ & $\begin{array}{l}73.12 \\
\pm 6.96\end{array}$ & $\begin{array}{l}64.92 \\
\pm 2.83\end{array}$ & $\begin{array}{l}64.45 \\
\pm 11.24\end{array}$ & $\begin{array}{l}68.03 \\
\pm 27.79\end{array}$ & $\begin{array}{l}64.94 \\
5.07\end{array}$ & $\begin{array}{l}64.07 \\
\pm 4.78\end{array}$ & $\begin{array}{l}54.69 \\
\pm 4.30\end{array}$ & $\begin{array}{l}13.63 \\
\pm 1.80\end{array}$ & $\begin{array}{l}16.01 \\
\pm 1.94\end{array}$ & $\begin{array}{l}77.99 \\
\pm \\
19.67\end{array}$ & $\begin{array}{l}46.11 \\
\pm 5.09\end{array}$ & $\begin{array}{l}41.23 \\
\pm 1.06\end{array}$ & $\begin{array}{l}22.11 \\
\pm 6.78\end{array}$ & $\begin{array}{l}63.06 \\
\pm 9.34\end{array}$ & $\begin{array}{l}61.38 \\
\pm 7.53\end{array}$ & $\begin{array}{l}78.62 \\
\pm 3.91\end{array}$ & $\begin{array}{l}55.89 \\
\pm 1.06\end{array}$ & $\begin{array}{l}46.21 \\
\pm .21 \\
13.64\end{array}$ & $\begin{array}{l}39.22 \\
\pm 1.24\end{array}$ & \begin{tabular}{|l|}
67.21 \\
\pm 3.30
\end{tabular} & $\begin{array}{l}55.81 \\
\pm 5.89\end{array}$ & $\begin{array}{l}49.14 \\
\pm 1.35\end{array}$ \\
\hline MI (\%) & $\begin{array}{l}2.3 \pm \\
0.5\end{array}$ & $\begin{array}{l}1.3 \pm \\
0.7\end{array}$ & $\begin{array}{l}1.2 \pm \\
0.5\end{array}$ & $\begin{array}{l}1.4 \pm \\
0.5\end{array}$ & $\begin{array}{l}1.1 \pm \\
0.2\end{array}$ & $\begin{array}{l}4.0 \pm \\
1.3\end{array}$ & $\begin{array}{l}1.9 \pm \\
0.2\end{array}$ & $\begin{array}{l}0.9 \pm \\
0.2\end{array}$ & $\begin{array}{l}1.3 \pm \\
0.2\end{array}$ & $\begin{array}{l}1.75 \pm \\
0.6\end{array}$ & $\begin{array}{l}2.5 \pm \\
0.6\end{array}$ & $\begin{array}{l}1.2 \pm \\
0.3\end{array}$ & $\begin{array}{l}1.7 \pm \\
0.6\end{array}$ & $\begin{array}{l}1.6 \pm \\
0.5\end{array}$ & $\begin{array}{l}1.4 \pm \\
0.5\end{array}$ & $\begin{array}{l}2.3 \pm \\
0.5\end{array}$ & $\begin{array}{l}1.3 \pm \\
0.7\end{array}$ & $\begin{array}{l}1.2 \pm \\
0.5\end{array}$ & $\begin{array}{l}1.4 \pm \\
0.5\end{array}$ & $\begin{array}{l}1.1 \pm \\
0.2\end{array}$ & \begin{tabular}{|l|}
$4.4 \pm$ \\
1.1
\end{tabular} & \begin{tabular}{|l|}
$1.7 \pm$ \\
0.5
\end{tabular} & $\begin{array}{l}1.6 \pm \\
0.4\end{array}$ & $\begin{array}{l}1.5 \pm \\
0.6\end{array}$ & $\begin{array}{l}1.6 \pm \\
0.3\end{array}$ & $\begin{array}{l}4.0 \pm \\
0.9\end{array}$ & $\begin{array}{l}1.9 \pm \\
0.5\end{array}$ & $\begin{array}{l}9.9 x \\
10^{-3} \pm \\
2.0 \times x \\
10^{-3} \\
\end{array}$ & $\begin{array}{l}4.5 x \\
10^{-3} \pm \\
6.1 \times \\
10^{-4} \\
\end{array}$ & $\begin{array}{l}6.7 \times \\
10^{-3} \pm \\
1.3 \times \\
10^{-3} \\
\end{array}$ \\
\hline$\mu\left(d^{-1}\right)$ & 0.026 & 0.018 & 0.017 & 0.019 & 0.016 & 0.035 & 0.023 & 0.014 & 0.018 & \begin{tabular}{|l|l|}
0.018 \\
\end{tabular} & 0.027 & 0.023 & 0.022 & 0.021 & 0.019 & 0.030 & 0.023 & 0.021 & 0.015 & 0.016 & 0.037 & \begin{tabular}{|l|l|}
0.022 \\
\end{tabular} & \begin{tabular}{|l|l|}
0.021 \\
\end{tabular} & 0.020 & 0.021 & 0.035 & 0.023 & $\frac{2 \times 10^{-}}{3}$ & $1 \times 10^{-4}$ & $2 \times 10^{-5}$ \\
\hline $\begin{array}{l}P_{\text {Gross }}(\mu \mathrm{g} \mathrm{C} \mathrm{mg-1} \\
\text { protein day }\end{array}$ & 0 & $\begin{array}{l}20.53 \\
\pm 3.88\end{array}$ & $\begin{array}{l}18.27 \\
\pm 5.40\end{array}$ & \begin{tabular}{|l|}
10.68 \\
\pm 0.73
\end{tabular} & \begin{tabular}{|l|}
10.88 \\
\pm 3.41
\end{tabular} & $\begin{array}{l}4.39 \pm \\
0.67\end{array}$ & $\begin{array}{l}30.14 \\
\pm 14.41\end{array}$ & $\begin{array}{l}5.79 \pm \\
1.99\end{array}$ & $\begin{array}{l}7.51 \pm \\
0.72\end{array}$ & \begin{tabular}{|l|}
$5.71 \pm$ \\
1.46
\end{tabular} & $\begin{array}{l}14.92 \\
\pm 6.27\end{array}$ & $\begin{array}{l}10.23 \\
\pm 1.60\end{array}$ & $\begin{array}{l}16.25 \\
\pm 5.56\end{array}$ & $\begin{array}{l}8.75 \pm \\
1.14\end{array}$ & \begin{tabular}{|l|}
29.02 \\
\pm \\
10.70
\end{tabular} & $\begin{array}{l}7.72 \pm \\
4.09\end{array}$ & $\begin{array}{l}9.48 \pm \\
2.01\end{array}$ & \begin{tabular}{|l|}
32.07 \\
\pm 9.68
\end{tabular} & $\begin{array}{l}7.25 \pm \\
0.58\end{array}$ & $\begin{array}{l}25.04 \\
\pm 8.10\end{array}$ & \begin{tabular}{|l|}
$2.62 \pm$ \\
0.89
\end{tabular} & \begin{tabular}{|l|}
$6.12 \pm$ \\
1.04
\end{tabular} & \begin{tabular}{|l|}
$8.79 \pm$ \\
0.77
\end{tabular} & $\begin{array}{l}5.22 \pm \\
1.96\end{array}$ & $\begin{array}{l}9.22 \pm \\
3.87\end{array}$ & $\begin{array}{l}7.01 \pm \\
2.99\end{array}$ & $\begin{array}{l}0.01 \pm \\
0.006\end{array}$ & $\begin{array}{l}5.48 \pm \\
2.02\end{array}$ & $\begin{array}{l}10.63 \\
\pm 0.52\end{array}$ & \begin{tabular}{|l|}
$5.41 \pm$ \\
0.71
\end{tabular} \\
\hline $\begin{array}{l}R_{\mathrm{s}}(\mu \mathrm{gg} \mathrm{C} \mathrm{mg-1} \\
\text { protein day }\end{array}$ & $\begin{array}{ll}22.23 \pm \\
4.52\end{array}$ & $\begin{array}{l}53.18 \\
\pm 5.89\end{array}$ & $\begin{array}{l}43.38 \pm \\
17.71\end{array}$ & \begin{tabular}{|l|}
16.28 \\
\pm 0.66
\end{tabular} & $\begin{array}{l}11.63 \\
\pm 2.40\end{array}$ & $\begin{array}{l}25.30 \\
\pm \\
14.96\end{array}$ & $\begin{array}{l}81.96 \\
\pm \\
32.05 \\
\end{array}$ & $\begin{array}{l}2.25 \pm \\
0.35\end{array}$ & $\begin{array}{l}8.36 \pm \\
1.31\end{array}$ & $\begin{array}{l}3.61 \pm \\
1.80\end{array}$ & $\begin{array}{l}35.96 \\
\pm \\
10.41\end{array}$ & $\begin{array}{l}46.62 \\
\pm 6.66\end{array}$ & $\begin{array}{l}20.34 \\
\pm 3.21\end{array}$ & $\begin{array}{l}12.53 \\
\pm 0.57\end{array}$ & \begin{tabular}{|l|}
46.58 \\
\pm \\
12.79 \\
\end{tabular} & $\begin{array}{l}41.16 \\
\pm 9.11\end{array}$ & $\begin{array}{l}17.62 \\
\pm 6.38\end{array}$ & \begin{tabular}{|l|}
154.84 \\
\pm \\
32.01 \\
\end{tabular} & $\begin{array}{l}16.83 \\
\pm 3.80\end{array}$ & $\begin{array}{l}45.13 \\
\pm \\
20.25 \\
\end{array}$ & $\begin{array}{l}10.12 \\
\pm 1.32\end{array}$ & \begin{tabular}{|l|}
26.68 \\
\pm 5.56
\end{tabular} & $\begin{array}{l}17.68 \\
\pm 5.28\end{array}$ & $\begin{array}{l}5.40 \pm \\
1.80\end{array}$ & $\begin{array}{l}6.36 \pm \\
1.00\end{array}$ & $\begin{array}{l}15.22 \\
\pm 6.71\end{array}$ & $\begin{array}{l}5.39 \pm \\
1.18\end{array}$ & $\begin{array}{l}9.19 \pm \\
3.46\end{array}$ & $\begin{array}{l}11.13 \\
\pm 1.99\end{array}$ & $\begin{array}{l}6.13 \pm \\
0.22\end{array}$ \\
\hline$P: R$ & 0 & 0.39 & 0.42 & 66 & 0.94 & 0.17 & 0.37 & 2.58 & 0.9 & 1.58 & 0.19 & 0.54 & 0.21 & 0.43 & 0.55 & 0.41 & 0.22 & \begin{tabular}{|l}
2.80 \\
0.80
\end{tabular} & 0.70 & 0.62 & 0.26 & 0.23 & 0.50 & 0.97 & 1.45 & 0.46 & 0.002 & 0.60 & 0.96 & \begin{tabular}{|l|}
0.88 \\
\end{tabular} \\
\hline $\begin{array}{l}\text { Algal:total } \\
\text { protein ratio } \times \\
10^{-2}\end{array}$ & $\begin{array}{l}0.89 \pm \\
0.25\end{array}$ & $\begin{array}{l}2.11 \pm \\
0.68\end{array}$ & $\begin{array}{l}14.37 \\
\pm 5.08\end{array}$ & \begin{tabular}{|l|}
41.74 \\
\pm 2.33 \\
\end{tabular} & $\begin{array}{l}15.93 \\
\pm 5.07\end{array}$ & $\begin{array}{l}0.42 \pm \\
0.11\end{array}$ & $\begin{array}{l}6.00 \pm \\
2.04\end{array}$ & $\begin{array}{l}15.66 \\
\pm 4.35\end{array}$ & $\begin{array}{l}23.21 \\
\pm 0.71\end{array}$ & $\begin{array}{l}5.45 \pm \\
0.88\end{array}$ & $\begin{array}{l}8.05 \pm \\
0.45\end{array}$ & $\begin{array}{l}5.24 \pm \\
1.75\end{array}$ & $\begin{array}{l}30.86 \\
\pm 3.01\end{array}$ & $\begin{array}{l}43.30 \\
\pm 6.77\end{array}$ & $\begin{array}{l}17.24 \\
\pm 1.73\end{array}$ & $\begin{array}{l}0.54 \pm \\
0.18\end{array}$ & $\begin{array}{l}1.40 \pm \\
0.64\end{array}$ & $\begin{array}{l}18.88 \\
\pm 3.17\end{array}$ & $\begin{array}{l}27.33 \\
\pm 1.97\end{array}$ & $\begin{array}{l}18.7 \pm \\
5.1\end{array}$ & $\begin{array}{l}1.48 \pm \\
0.70\end{array}$ & \begin{tabular}{|l}
11.32 \\
\pm 2.38
\end{tabular} & $\begin{array}{l}46.65 \\
\pm 4.57\end{array}$ & $\begin{array}{l}39.38 \\
\pm \\
18.84\end{array}$ & $\begin{array}{l}6.56 \pm \\
0.14\end{array}$ & $\begin{array}{l}0.19 \pm \\
0.06\end{array}$ & $\begin{array}{l}0.72 \pm \\
0.36\end{array}$ & \begin{tabular}{|l|}
13.20 \\
\pm 3.09
\end{tabular} & $\begin{array}{l}8.55 \pm \\
2.86\end{array}$ & $\begin{array}{l}3.53 \pm \\
0.97\end{array}$ \\
\hline $\begin{array}{l}R_{a}(\mu \mathrm{g} \mathrm{C} \mathrm{mg-1} \\
\text { protein day }\end{array}$ & $\begin{array}{l}22.05 \\
\pm 4.50\end{array}$ & $\begin{array}{l}52.21 \\
\pm 5.17\end{array}$ & $\begin{array}{l}31.63 \pm \\
13.36\end{array}$ & \begin{tabular}{|l|}
$11.14 \pm$ \\
1.34
\end{tabular} & $\begin{array}{l}9.34 \pm \\
1.02\end{array}$ & $\begin{array}{l}25.23 \\
\pm \\
12.96\end{array}$ & $\begin{array}{l}80.73 \\
\pm \\
32.56 \\
\end{array}$ & \begin{tabular}{|l|}
$1.77 \pm$ \\
0.29
\end{tabular} & $\begin{array}{l}6.17 \pm \\
0.84\end{array}$ & \begin{tabular}{|l|}
$3.43 \pm$ \\
0.56
\end{tabular} & $\begin{array}{l}33.50 \\
\pm 9.87\end{array}$ & $\begin{array}{l}43.66 \\
\pm 3.77\end{array}$ & $\begin{array}{l}11.39 \\
\pm 2.21\end{array}$ & \begin{tabular}{|l|}
$8.28 \pm$ \\
0.89
\end{tabular} & $\begin{array}{l}39.29 \\
\pm 9.70\end{array}$ & $\begin{array}{l}40.19 \\
\pm 9.13\end{array}$ & $\begin{array}{l}17.42 \\
\pm 5.39\end{array}$ & \begin{tabular}{|l|}
117.27 \\
\pm \\
14.85
\end{tabular} & $\begin{array}{l}11.87 \\
\pm 2.82\end{array}$ & $\begin{array}{l}44.56 \\
\pm \\
17.82\end{array}$ & \begin{tabular}{|l|}
10.05 \\
\pm 1.14 \\
\end{tabular} & \begin{tabular}{|l|}
25.85 \\
\pm 4.56
\end{tabular} & \begin{tabular}{|l|}
10.88 \\
\pm 4.52 \\
\end{tabular} & $\begin{array}{l}4.50 \pm \\
1.64\end{array}$ & $\begin{array}{l}5.78 \pm \\
0.74\end{array}$ & $\begin{array}{l}15.21 \\
\pm 5.81\end{array}$ & $\begin{array}{l}5.35 \pm \\
1.32\end{array}$ & $\begin{array}{l}7.25 \pm \\
2.61\end{array}$ & $\begin{array}{l}9.98 \pm \\
1.76\end{array}$ & \begin{tabular}{|l|}
$5.91 \pm$ \\
0.18
\end{tabular} \\
\hline $\begin{array}{l}\mathbf{R}_{z}\left(\mu \mathrm{gg} \mathrm{Cg}^{-1}\right. \\
\text { protein day }\end{array}$ & $\begin{array}{l}0.18 \pm \\
0.05\end{array}$ & \begin{tabular}{|l|}
$0.71 \pm$ \\
0.26
\end{tabular} & $\begin{array}{l}13.30 \pm \\
2.64\end{array}$ & $\begin{array}{l}14 \pm \\
42\end{array}$ & $\begin{array}{l}2.29 \pm \\
1.25\end{array}$ & $\begin{array}{l}0.04 \pm \\
0.008\end{array}$ & $\begin{array}{l}1.63 \pm \\
0.64\end{array}$ & $\begin{array}{l}0.35 \pm \\
0.10\end{array}$ & $\begin{array}{l}2.20 \pm \\
0.57\end{array}$ & 20 & $\begin{array}{l}2.46 \pm \\
0.96\end{array}$ & $\begin{array}{l}96 \pm \\
13\end{array}$ & $\begin{array}{l}4.05 \pm \\
0.58\end{array}$ & 14 & $\begin{array}{l}7.30 \pm \\
1.85\end{array}$ & $\begin{array}{l}0.18 \pm \\
0.07\end{array}$ & $.29 \pm$ & \begin{tabular}{|l|}
37.56 \\
\pm \\
14.14 \\
\end{tabular} & $\begin{array}{l}4.96 \pm \\
0.58\end{array}$ & $\begin{array}{l}0.57 \pm \\
0.39\end{array}$ & $\begin{array}{l}0.07 \pm \\
0.04\end{array}$ & \begin{tabular}{|l|}
$0.83 \pm$ \\
0.40
\end{tabular} & $\begin{array}{l}79 \pm \\
57\end{array}$ & $89 \pm$ & $\begin{array}{l}0.58 \pm \\
0.19\end{array}$ & $\begin{array}{l}0.01 \pm \\
0.007\end{array}$ & $\begin{array}{l}0.05 \pm \\
0.03\end{array}$ & $.95 \pm$ & $\begin{array}{l}1.14 \pm \\
0.61\end{array}$ & \begin{tabular}{|l|}
$0.24 \pm$ \\
0.08
\end{tabular} \\
\hline $\begin{array}{l}\text { Algal carbon } \\
\text { standing stock: } \\
\mathrm{C}^{\prime}\left(\mu \mathrm{gg} \mathrm{mg}^{-1}\right. \\
\text { protein) }\end{array}$ & $\begin{array}{l}0.95 \pm \\
0.27\end{array}$ & $\begin{array}{l}2.29 \pm \\
0.75\end{array}$ & $\begin{array}{l}32.65 \\
\pm \\
15.13\end{array}$ & \begin{tabular}{|l|}
33.39 \\
\pm 7.77
\end{tabular} & $\begin{array}{l}21.33 \\
\pm 8.21\end{array}$ & $\begin{array}{l}0.43 \pm \\
0.12\end{array}$ & $\begin{array}{l}6.85 \pm \\
2.45\end{array}$ & $\begin{array}{l}43.83 \\
\pm \\
18.80\end{array}$ & $\begin{array}{l}30.60 \\
\pm 1.84\end{array}$ & \begin{tabular}{|l|}
$6.11 \pm$ \\
1.04
\end{tabular} & $\begin{array}{l}12.24 \\
\pm 3.02\end{array}$ & $\begin{array}{l}5.94 \pm \\
2.07\end{array}$ & $\begin{array}{l}57.16 \\
\pm \\
10.72\end{array}$ & $\begin{array}{l}33.66 \\
\pm 4.85\end{array}$ & \begin{tabular}{|l|}
22.15 \\
\pm 2.74
\end{tabular} & \begin{tabular}{|l|}
$0.57 \pm$ \\
0.19
\end{tabular} & $\begin{array}{l}1.51 \pm \\
0.70\end{array}$ & \begin{tabular}{|l}
34.91 \\
\pm 12.59 \\
12.59
\end{tabular} & $\begin{array}{l}39.99 \\
\pm 3.98\end{array}$ & $\begin{array}{l}7.03 \pm \\
1.44\end{array}$ & \begin{tabular}{|l|}
$0.74 \pm$ \\
0.34
\end{tabular} & \begin{tabular}{|l|}
$4.75 \pm$ \\
1.90
\end{tabular} & \begin{tabular}{|l}
39.45 \\
\pm 17.67 \\
17.67
\end{tabular} & $\begin{array}{l}46.66 \\
\pm \pm \\
19.41\end{array}$ & $\begin{array}{l}7.48 \pm \\
1.72\end{array}$ & $\begin{array}{l}0.20 \pm \\
0.07\end{array}$ & $\begin{array}{l}0.47 \pm \\
0.22\end{array}$ & \begin{tabular}{|l|}
19.68 \\
\pm 3.77
\end{tabular} & $\begin{array}{l}10.22 \\
\pm 3.72\end{array}$ & \begin{tabular}{|l|}
$3.89 \pm$ \\
1.12
\end{tabular} \\
\hline 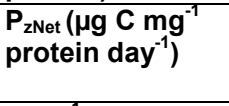 & 0 & \begin{tabular}{|l|}
34.62 \\
\pm \\
13.58 \\
\end{tabular} & $\begin{array}{l}31.32 \\
\pm \\
14.65 \\
\end{array}$ & \begin{tabular}{|l|}
$8.94 \pm$ \\
0.68
\end{tabular} & \begin{tabular}{|l}
$9.50 \pm$ \\
3.12
\end{tabular} & $\begin{array}{l}3.87 \pm \\
0.95\end{array}$ & $\begin{array}{l}9.93 \pm \\
1.66\end{array}$ & $\begin{array}{l}5.29 \pm \\
1.49\end{array}$ & $\begin{array}{l}3.12 \pm \\
0.73\end{array}$ & \begin{tabular}{|l|}
$5.07 \pm$ \\
1.25
\end{tabular} & $\begin{array}{l}7.50 \pm \\
4.38\end{array}$ & $\begin{array}{l}8.18 \pm \\
2.49\end{array}$ & $\begin{array}{l}15.09 \\
\pm 5.53\end{array}$ & $\begin{array}{l}13.62 \\
\pm 5.95\end{array}$ & \begin{tabular}{|l|}
26.96 \\
\pm \\
10.15 \\
\end{tabular} & $\begin{array}{l}1.50 \pm \\
0.91\end{array}$ & $\begin{array}{l}9.43 \pm \\
2.33\end{array}$ & $\begin{array}{l}8.21 \pm \\
2.04\end{array}$ & $\begin{array}{l}6.72 \pm \\
0.68\end{array}$ & $\begin{array}{l}19.60 \\
\pm 5.95\end{array}$ & \begin{tabular}{|l|}
$2.60 \pm$ \\
0.89
\end{tabular} & $\begin{array}{l}3.93 \pm \\
1.69\end{array}$ & \begin{tabular}{|l|}
$8.11 \pm$ \\
1.84
\end{tabular} & $\begin{array}{l}4.94 \pm \\
2.03\end{array}$ & \begin{tabular}{|l|}
$8.82 \pm$ \\
3.70
\end{tabular} & $\begin{array}{l}6.84 \pm \\
2.12\end{array}$ & $\begin{array}{l}2.16 \pm \\
0.89\end{array}$ & \begin{tabular}{|l|}
$5.48 \pm$ \\
2.02
\end{tabular} & $\begin{array}{l}8.78 \pm \\
1.90\end{array}$ & \begin{tabular}{|l|}
$5.41 \pm$ \\
0.71
\end{tabular} \\
\hline$\mu_{c}\left(d^{-1}\right)$ & 0 & $\begin{array}{l}18.34 \\
\pm 4.82\end{array}$ & $\begin{array}{l}0.88 \pm \\
0.50\end{array}$ & $\begin{array}{l}0.30 \pm \\
0.05\end{array}$ & \begin{tabular}{|l|l|}
$0.49 \pm$ \\
0.09
\end{tabular} & $\begin{array}{l}17.72 \\
\pm 7.02\end{array}$ & $\begin{array}{l}3.61 \pm \\
1.05\end{array}$ & \begin{tabular}{|l|}
$0.16 \pm$ \\
0.06
\end{tabular} & $\begin{array}{l}0.27 \pm \\
0.12\end{array}$ & \begin{tabular}{|l|}
$0.83 \pm$ \\
0.14
\end{tabular} & $\begin{array}{l}0.10 \pm \\
0.05\end{array}$ & $\begin{array}{l}0.02 . \pm \\
0.01\end{array}$ & $\begin{array}{l}0.23 \pm \\
0.04\end{array}$ & \begin{tabular}{|l}
$0.50 \pm$ \\
0.17
\end{tabular} & $\begin{array}{l}1.20 \pm \\
0.41\end{array}$ & $\begin{array}{l}48.09 \\
\pm \\
22.62\end{array}$ & $\begin{array}{l}24.87 \\
\pm 9.23\end{array}$ & \begin{tabular}{|l}
$0.79 \pm$ \\
0.15
\end{tabular} & $\begin{array}{l}0.17 \pm \\
0.02\end{array}$ & $\begin{array}{l}1.63 \pm \\
0.43\end{array}$ & $\begin{array}{l}5.35 \pm \\
2.69\end{array}$ & \begin{tabular}{|l|}
$0.83 \pm$ \\
0.07
\end{tabular} & \begin{tabular}{|l|}
$0.36 \pm$ \\
0.13
\end{tabular} & \begin{tabular}{|l|}
$0.32 \pm$ \\
0.01
\end{tabular} & $\begin{array}{l}1.08 \pm \\
0.19\end{array}$ & $\begin{array}{l}28.86 \\
\pm 9.76\end{array}$ & $\begin{array}{l}0.59 \pm \\
0.05\end{array}$ & \begin{tabular}{|l|}
$0.35 \pm$ \\
0.06
\end{tabular} & $\begin{array}{l}0.82 \pm \\
0.30\end{array}$ & $\begin{array}{l}1.71 \pm \\
0.43\end{array}$ \\
\hline $\mathrm{T}(\%)$ & 0 & \begin{tabular}{|l}
99.33 \\
\pm 0.54
\end{tabular} & $\begin{array}{l}96.27 \pm \\
2.26\end{array}$ & \begin{tabular}{|l|}
92.17 \\
\pm 1.13
\end{tabular} & $\begin{array}{l}96.43 \\
\pm 0.55\end{array}$ & $\begin{array}{l}99.54 \\
\pm 0.25\end{array}$ & $\begin{array}{l}98.91 \\
\pm 0.55\end{array}$ & $\begin{array}{l}90.18 \\
\pm 4.15\end{array}$ & $\begin{array}{l}84.29 \\
\pm 5.52\end{array}$ & $\begin{array}{l}97.50 \\
\pm 0.59\end{array}$ & $\begin{array}{l}86.74 \\
\pm 6.54\end{array}$ & $\begin{array}{l}88.85 \\
\pm \\
23.54 \\
\end{array}$ & $\begin{array}{l}89.73 \\
\pm 1.92\end{array}$ & $\begin{array}{l}83.20 \\
\pm \\
10.43 \\
\end{array}$ & \begin{tabular}{|l|}
97.35 \\
\pm 1.41
\end{tabular} & \begin{tabular}{|l}
96.41 \\
\pm 3.25
\end{tabular} & $\begin{array}{l}99.75 \\
\pm 0.17\end{array}$ & \begin{tabular}{|l}
98.32 \\
\pm 1.58
\end{tabular} & $\begin{array}{l}91.19 \\
\pm 1.50\end{array}$ & \begin{tabular}{|l|}
98.74 \\
\pm 0.55
\end{tabular} & \begin{tabular}{|l}
98.78 \\
\pm 0.44
\end{tabular} & \begin{tabular}{|l}
97.33 \\
\pm 0.23
\end{tabular} & $\begin{array}{l}85.83 \\
\pm 9.98\end{array}$ & $\begin{array}{l}70.00 \\
\pm \\
14.82 \\
\end{array}$ & \begin{tabular}{|l}
97.93 \\
\pm 0.37
\end{tabular} & $\begin{array}{l}99.85 \\
\pm 0.07\end{array}$ & $\begin{array}{l}95.98 \\
\pm 3.76\end{array}$ & \begin{tabular}{|l}
99.89 \\
\pm 0.04
\end{tabular} & $\begin{array}{l}99.98 \\
\pm 0.01\end{array}$ & \begin{tabular}{|l|}
99.99 \\
\pm \\
0.001
\end{tabular} \\
\hline CZAR (\%) & 0 & $\begin{array}{l}29.66 \pm \\
8.33\end{array}$ & 11 & \pm 3.14 & \begin{tabular}{|l}
74.92 \\
\pm \\
12.06
\end{tabular} & \pm 5.46 & \pm 8.47 & $\begin{array}{l}150.93 \\
\pm \\
60.76\end{array}$ & $\begin{array}{l}62.68 \\
\pm \\
29.27\end{array}$ & \begin{tabular}{|l}
1112.98 \\
\pm \\
10.85
\end{tabular} & \pm 8.40 & $\begin{array}{l}8.58 \pm \\
3.04\end{array}$ & $\begin{array}{l}63.07 \\
\pm \\
19.18\end{array}$ & $\begin{array}{l}50.47 \\
\pm \\
12.84\end{array}$ & \begin{tabular}{|l|l}
72.44 \\
\pm \\
26.17
\end{tabular} & \pm 5.46 & $\begin{array}{l}52.57 \\
\pm \\
35.40\end{array}$ & 02 & $\begin{array}{l}40.58 \\
\pm \\
10.44\end{array}$ & \pm 8.34 & \pm 7.21 & \pm 3.42 & \begin{tabular}{|l}
499.97 \\
\pm \\
26.34
\end{tabular} & $\begin{array}{l}100.95 \\
\pm 0.57\end{array}$ & \begin{tabular}{|l|l}
176.77 \\
\pm \\
26.11
\end{tabular} & \pm 1.45 & $\begin{array}{l}22.82 \\
\pm \\
11.51\end{array}$ & $\begin{array}{l}46.22 \\
\pm 8.20\end{array}$ & $\begin{array}{l}91.66 \\
\pm \\
35.80\end{array}$ & \begin{tabular}{|l|}
81.10 \\
\pm 7.05
\end{tabular} \\
\hline
\end{tabular}


Appendix 2.

Measuring the host cell pHi in $A$. pulchella-Symbiodinium associations with different symbiont types

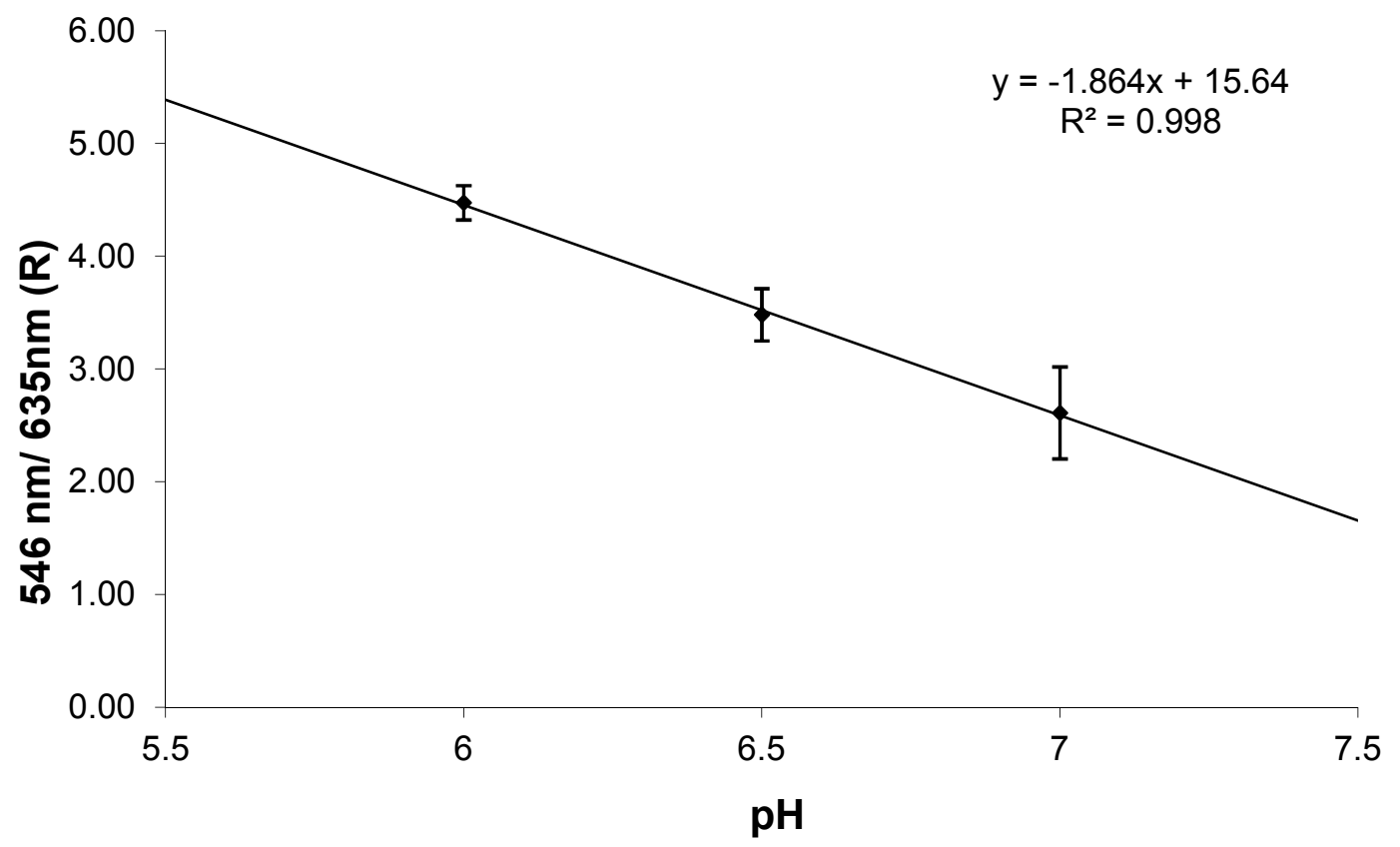

Figure A2.1. Calibration curve of the internal cellular $\mathrm{pH}$ (pHi) of Aiptasia pulchella cells with the SNARF-4f signal. 
Appendix

Appendix 3.

\section{ITS2 sequences of Symbiodinium types used in this study}

The Symbiodinium types ( $\mathrm{n}=3$ samples per culture or isolate) used in the experiments in Chapter 2, 3 and 4 were genetically identified prior to infection of A. pulchella. After 12 weeks, zooxanthellae were isolated from three anemones per treatment and their genetic identity confirmed, using the isolation and molecular techniques described in the methods section of each chapter. All treatments were found to harbour the same Symbiodinium type as the one introduced at the start of the experiment. Both the freshly-isolated and cultured homolgous Symbiodinium B1, and the heterologous Symbiodinium B1 had identical ITS2 sequences. Table A3.1 shows the NCBI accession numbers of the ITS2 sequences that were used to identify the Symbiodinium types used in this study.

Sequences of the 5.8s ribosomal RNA gene (partial sequence), internal transcribed spacer ITS2 (complete sequence) and 28s ribosomal RNA gene (partial sequence) for Symbiodinium sub-clades (A) A1.4, (B) B1, (C) E2 and (D) F5.1.

(A)

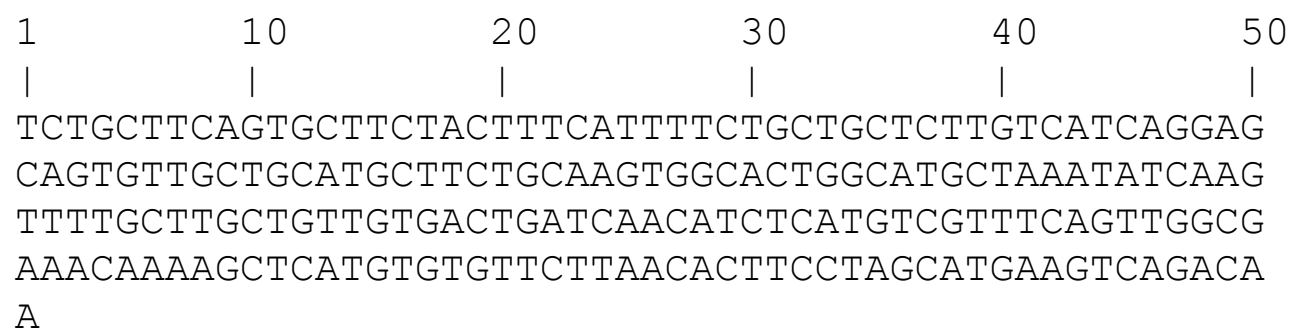

(B)

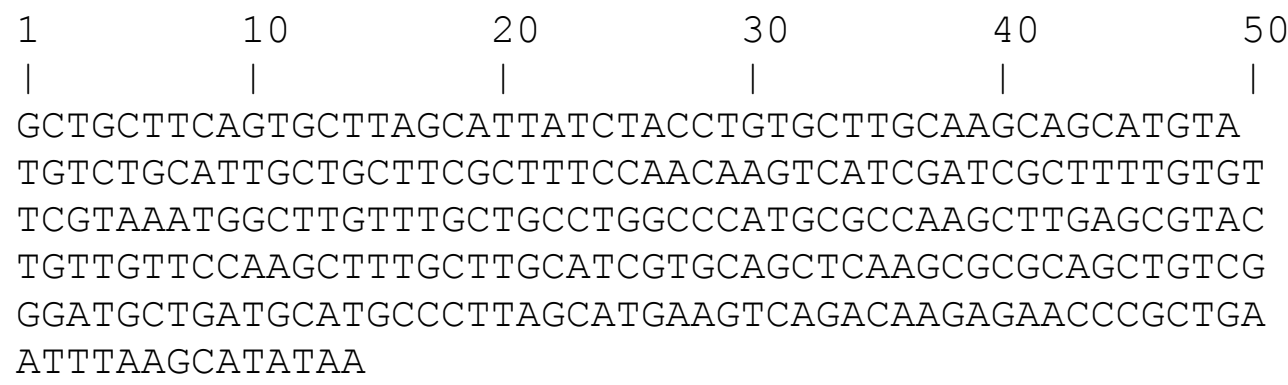


Appendix

(C)

$\begin{array}{cccccc}1 & 10 & 20 & 30 & 40 & 50 \\ 1 & 1 & \mid & 1 & \mid & \mid\end{array}$

TCTGCTTCAGTGCTTTTCATATCTTCGCAGTGCGGGCTTCCTGGAGAAGC

CTTGAGCCTCTTTGCGCGCTGCTGCATCAGAATTTGCAGCGGCGCGCTGA

ACACAAACCGGGAGGTAAGCTGGACTGATTTGTCGCGCATCACTGGGCAC

GTGTGTCCGTTTTGGCCCAATCATGCCAGCCTGCCAAGCAATTGGTGCTC

AAATACCAATCTTAGCATGAAGTCAGACAAGCAAACCCGCTGAATTTAAG

CATATAA

(D)
1
10
20
30
40
50
I 1
।
।
I
TCTGCTTCAGTGCTTAGCTTGCCCAATCTTGCGGATAGACTTTGTTTCTG
TCTTGCGCCCCTGTGAGCCATTGAGCGTCTAGTCAATGGCTTATTGAATG
ATTCGGTCTTGCAAAAGCTTTGCGCGCTTCTATTCAAGATTCCACCTTGG
AGTGGTATTGCTTGAGTGACGCTGCTCATGCTTGCAACTGCTGGGATGCT
AACGCATGCCTCTAGCATGAAGTCAGACAAGCGAACCCGCTGAATTTAAG
CATATAA

Table A3.1. NCBI accession numbers of the ITS2 sequences that were used to identify the Symbiodinium types used in this study.

\begin{tabular}{|c|c|c|}
\hline Sequence & Symbiodinium type & $\begin{array}{c}\text { NCBI accession } \\
\text { number }\end{array}$ \\
\hline A & Symbiodinium A1.4 & HE578977 \\
\hline B & Symbiodinium B1 & EU074875 \\
\hline C & Symbiodinium E2 & JN558086 \\
\hline D & Symbiodinium F5.1 & AM748615 \\
\hline
\end{tabular}


Appendix

\section{Appendix 4.}

\section{Symbiosome membrane isolation and proteomics}

\section{A4.1. Introduction}

The symbiotic lifestyle involves mutual ecological, physiological, structural, and molecular adaptations between the partners, and mechanisms that allow for the recognition of the symbiont by the host (or vice versa) as well as for the establishment and persistence of the symbiosis. However, not much is known about these mechanisms (Furla et al., 2005). Because the symbiosome membrane complex acts as a barrier between the host cell cytoplasm and the symbiont (Wakefield and Kempf, 2001), an understanding of the communicative processes between the algal symbiont and its cnidarian partner, which are no doubt crucial to the nutrition, stability and persistence of the symbiotic relationship, is necessary. It is essential to characterise the properties of the symbiosome membrane as the transport properties of this membrane govern what passes between the host and symbiont. Thus there is a level of specificity as to which symbiont type can successfully colonise the host resulting in an "effective" symbiosis. Kazandjian et al, (2008) isolated the symbiosome membrane from the zoanthid, Zoanthus robustus, using a combination of mechanical disruption and sucrose gradient centrifugation. Using confocal microscopy and transmission electron microscopy (TEM) they confirmed that the symbiosome interface surrounds the alga and also established that it is multilayered, robust and displays a level of heterogeneity in terms of thickness. More studies aimed at examining the membrane composition and membrane trafficking using fluorescent markers are necessary to gain an understanding of the symbiosomemembrane modification that occurs during symbiosis. Questions that need to be addressed include: How does transport across the membrane occur? Does the symbiosome interface have transporter/protein pores, dicarboxylate transporters and glucose transporters to facilitate this transport, and is the transport active or passive? Can the transporters be up- and down-regulated and is there transcription-level 
Appendix

regulation of the transport proteins? Furthermore, what happens to the symbiosome membrane when the symbiotic alga within divides? Does this membrane divide simultaneously with the alga? How is the division of the symbiosome membrane coordinated, so that overgrowth of the symbiosome by dividing algal symbionts is prevented? For how long can a symbiosome membrane lineage persist? Is the level of coordination maintained with all types of algal symbiont? The focus of this study was to address some of these key questions. Unfortunately, despite progressing to the point of successfully isolating the symbiosome membrane complex from the $A$. pulchella-Symbiodinium association and isolating the membrane proteins, due to funding and time constraints, the membrane proteomic work could not be completed.

The aim of the study was to characterise the symbiosome membrane complex and the impacts of symbiosis establishment on its functional properties.

Specifically, the objectives and hypotheses were:

1. To isolate the symbiosome membrane complex from $A$. pulchella using a modified version of the method of Kazandjian (2008).

2. To identify the key nutrient transporters present in the symbiosome membrane.

a. Key nutrient transporters will be upregulated during the infection process.

3. To compare the key nutrient transporters present in the symbiosome membrane amongst symbioses incorporating different symbionts.

a. The upregulation of nutrient transporters will vary amongst the different symbioses.

b. Symbioses incorporating homologous symbionts will show greater upregulation of key nutrient transporters. 
Appendix

\section{A4.2. Materials and methods}

\section{A4.2.1. Symbiosome membrane isolation}

Four $A$. pulchella anemones were homogenized in $1 \mathrm{ml}$ of $0.44-\mu \mathrm{m}$ FSW in a tissue grinder and centrifuged at $\times 400 \mathrm{~g}$. The supernatant was removed and the process repeated twice. The animal tissue was then mechanically disrupted to release symbiosomes by passing the extract vigorously seven times through a 23-gauge needle ( $0.33 \mathrm{~mm}$ inner diameter) into a Falcon tube. Symbiosomes were separated from gastrodermal cell debris, cnidoblasts and other animal cells by sucrose gradient density centrifugation. The deaggregated and broken gastrodermal homogenate was loaded onto a step-wise sucrose gradient $(30 \%-80 \%)$. The gradient was centrifuged at $\times 3893 \mathrm{~g}$ for 1 hour (Fig.) with slow acceleration/deceleration rates, at $12-15^{\circ} \mathrm{C}$. Gently disrupted material resolved into three bands of symbiosomes on the sucrose gradients: top, above $40 \%$ sucrose, middle, above 50\% sucrose, and bottom, above $60 \%$ sucrose. Vigorously disrupted material resolved into a single large band above $40 \%$ sucrose. All symbiosome bands were were stainedwith FM 1-43 and assessed for purity using fluorescence and bright-field microscopy as described below. The fractions containing symbiosomes were then pooled diluted in PBS-50\%FSW, pelleted (at $\times 3893 \mathrm{~g}$ for $30 \mathrm{~min}$ ) and resuspended in $\mathrm{PBS}-50 \% \mathrm{FSW}$. The resuspended symbiosomes were vigorously disrupted, by strong manual pressure on, and seven repeat passes through, the 23 gauge needle described above, loaded on to a second sucrose gradient $(30 \%-80 \%)$. and centrifuged as described previously. The symbiosome membrane fraction was recovered as a golden layer of vesicles suspended in the load zone. The golden-layer material derived from both first and second sucrose gradients were stained with FM 1-43 and examined using fluorescence microscopy as described below.

\section{A4.2.2. Symbiosome and symbiosome membrane visualisation}

Fractions containing symbiosomes and the symbiosome membrane were fluorescence-labelled with FM 1-43 (Molecular Probes, U. S. A.) $\left(\lambda_{\mathrm{ex}} 479 \mathrm{~nm}, \lambda_{\mathrm{em}}\right.$ $598 \mathrm{~nm}$ ). FM 1-43 is a vitally staining fluorochrome in the styryl family, used for 
Appendix

detecting vesicle trafficking in eukaryotic cells (Bolte et al., 2004). FM 1-43 was selected for labelling the symbiosome membrane because it identifies internalised orendocytosed membranes derived from the plasma membrane (Bolte et al., 2004). Stock solutions of FM 1-43 were made up in Milli-Q water at a concentration of 1 $\mathrm{mM}$, and were kept in the dark at $-20{ }^{\circ} \mathrm{C}$. The $40 \%$ sucrose fraction (the symbiosome-enriched fraction) was collected and stained for 10 min with $30 \mu \mathrm{M} \mathrm{FM}$ 1-43 and10 $\mu \mathrm{g} \mathrm{ml}^{-1}$ Hoechst 33342 for visualisation of the symbiosome membrane and nuclear material respectively. FM 1-43 and Hoechst 3342 fluorescence was observed with fluorescence microscopy (Olympus Provis AX70 microscope) at 100× magnification using a FITC filter to visualize FM 1-43 fluorescence and a DAPI filter to visualise Hoechst 33342 fluorescence.

\section{A4.2.3. Digestion of isolated membranes}

The symbiosome membranes fractions were pooled together. A $500 \mu \mathrm{L}$ aliquot of the pooled fractions was incubated with $500 \mu \mathrm{L}$ of $2 \%$ Triton X-100 (prepared in FSW), $40 \mu \mathrm{L}$ of protease inhibitor cocktail (Roche Diagnostics, Manheim, Germany, Cat. No. 11697498001) and $5 \mu \mathrm{L}$ of saturated $\mathrm{NaCl}$ solution (Sigma-Aldrich, Auckland). The solution was then mixed in a rotating mixer (rotating $90^{\circ}$ right and left) for 30 min at room temperature. The mixture was centrifuged at $\times 50 \mathrm{~g}$ for $5 \mathrm{~min}$ in order to separate the Triton X-100-soluble fraction (i.e., the supernatant) from the insoluble fraction (i.e., the pellet). The Triton X-100-soluble fraction was collected for subsequent trichloroacetic acid (TCA) protein precipitation. The Triton X-100insoluble pellets containing the membrane fractions were resuspended in $1 \mathrm{ml}$ RIPA buffer (50 mM Tris, $\mathrm{pH} 7.4,1 \%$ NP-40, 0.25\% Na-deoxycholate, $150 \mathrm{mM} \mathrm{NaCl}, 1$ mM EDTA, $1 \mathrm{mM} \mathrm{Na} \mathrm{VO}_{3}, 1 \mathrm{mM} \mathrm{NaF}$ ) and $40 \mu \mathrm{L}$ protease inhibitor cocktail. The solution was homogenized in liquid nitrogen and the homogenate centrifuged for 5 min at $\times 50 \mathrm{~g}$ to obtain the supernatant in preparation for TCA protein precipitation. All dyes used for microscopy were purchased from Invitrogen (Auckland); other chemicals were purchased from Sigma-Aldrich (Auckland) unless otherwise stated. 
Appendix

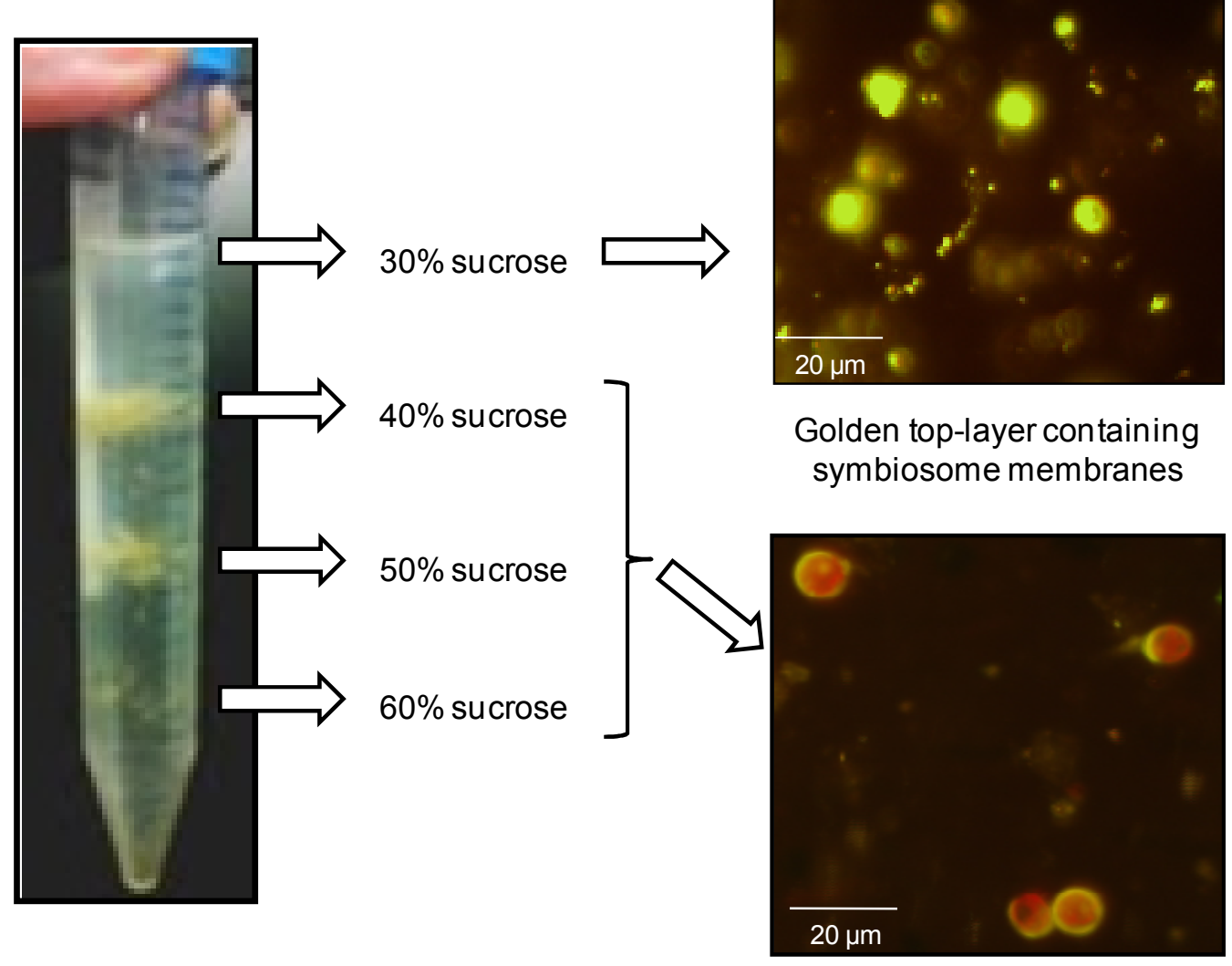

Symbiosome en riched fractions

Figure A4.1. Photograph of the step-wise sucrose gradient used to isolate the symbiosome membrane. The various bands obtained include the golden layer enriched with symbiosome membranes above $30 \%$ sucrose, and the symbiosome enriched layers above $40 \%, 50 \%$ and $60 \%$ sucrose. The top fluorescence microscope image depicts FM 1-43 stained symbiosome membrane complex fragments. The bottom fluorescence image depicts FM 1-43 stained symbiosomes (the symbiosome membrane stains yellow). 